\title{
Modelling genetic heart diseases with patient-specific induced pluripotent stem cells
}

\author{
Dissertation \\ for the award of the degree
}

'Doctor rerum naturalium'

of the Georg-August-Universität Göttingen

within the doctoral program 'Genes and Development'

of the Georg-August-University School of Science (GAUSS)

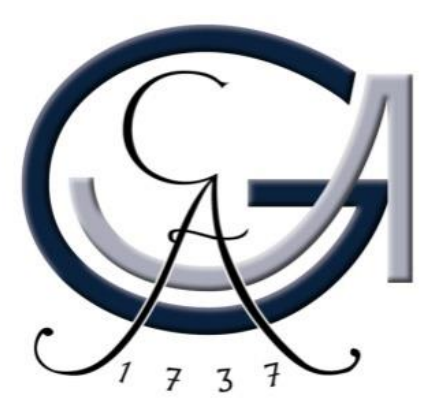

submitted by

Michael Stauske

from Leipzig

Göttingen 2014 



\section{Thesis committee members}

Prof. Dr. Ernst A. Wimmer $\left(1^{\text {st }}\right.$ reviewer $)$

Department of Developmental Biology

Johann-Friedrich-Blumenbach-Institute of Zoology and Anthropology Göttingen

Prof. Dr. med. Dr. h. c. Wolfgang Engel ( $2^{\text {nd }}$ reviewer $)$

Institute of Human Genetics

University Medical Center Göttingen

PD Dr. Kaomei Guan-Schmidt

Department of Cardiology and Pneumology

University Medical Center Göttingen

\section{Additional members of the examination board}

Prof. Dr. med. Michael Zeisberg

Department of Nephrology and Rheumatology

University Medical Center Göttingen

\section{PD Dr. Viacheslav Nikolaev}

Department of Cardiology and Pneumology

University Medical Center Göttingen

\section{Dr. Roland Dosch}

Department of Developmental Biochemistry

University Medical Center Göttingen

\section{Date of oral examination}

June $18^{\text {th }}, 2014$ 



\section{Table of contents}

List of abbreviations ............................................................................................

1 Introduction.................................................................................................................. 1

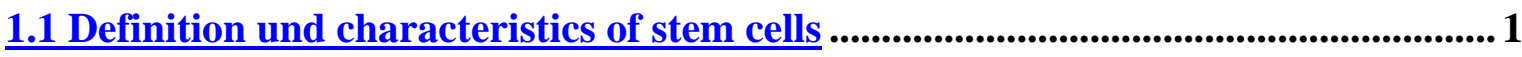

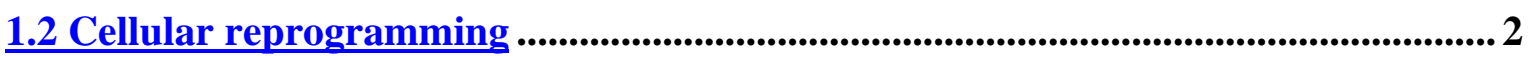

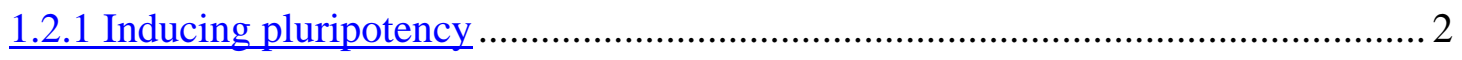

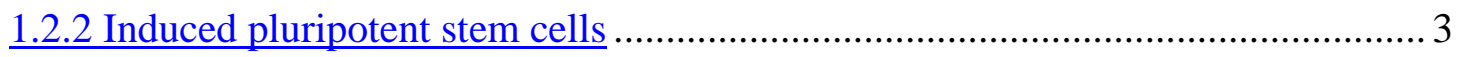

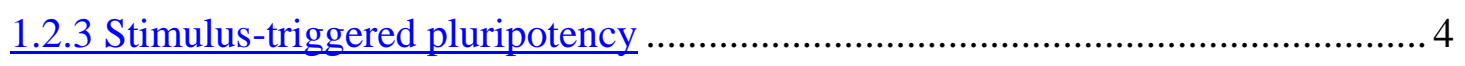

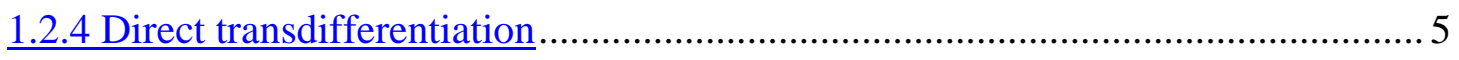

1.3 Cardiac electrophysiology and arrhythmias ...................................................................5

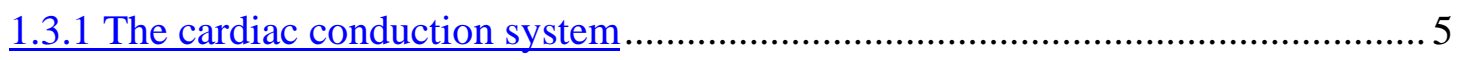

1.3.2 Action potentials and excitation-contraction coupling ................................... 7

1.3.3 Structure, function, and regulation of the cardiac sodium channel .................... 9

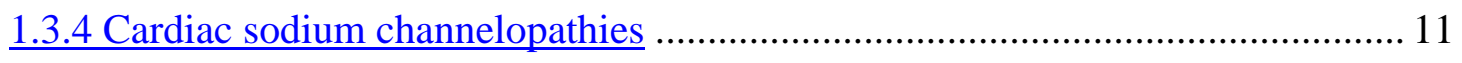

1.3.4.1 SCN5A mutations and Long-QT syndrome ..................................... 13

1.3.4.2 SCN5A mutations and Brugada syndrome …..................................... 13

\subsubsection{Model systems for studying SCN5A mutation-caused}

channelopathies ….................................................................... 14

1.4 Application of human iPSCs in cardiac research ............................................................ 16

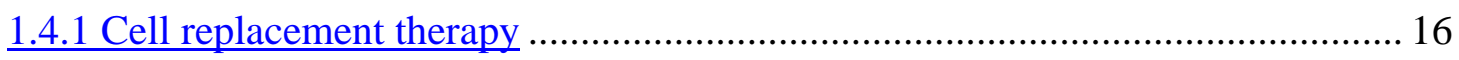

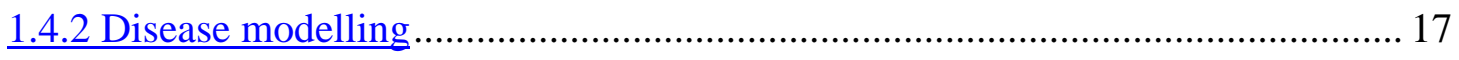

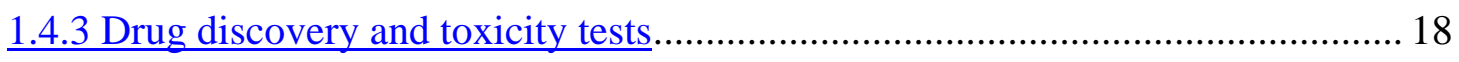

1.5 Aim of this thesis ........................................................................................................................... 19 


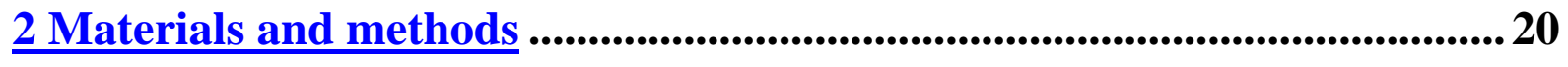

2.1 Materials ...................................................................................................................................20

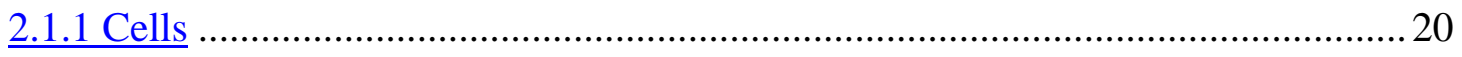

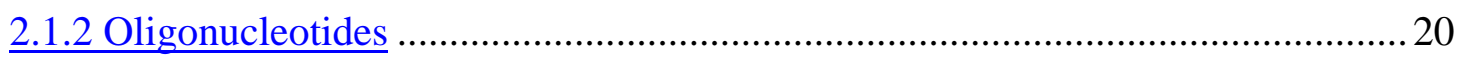

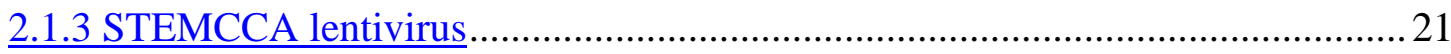

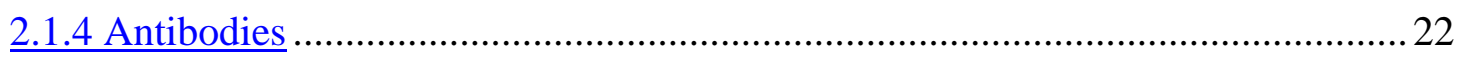

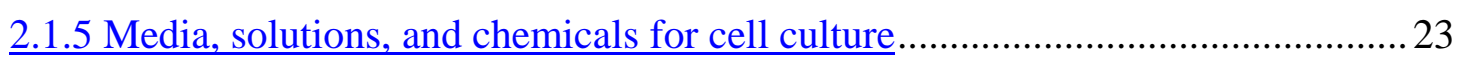

2.1.6 Solutions, buffers, and chemicals for molecular biological,

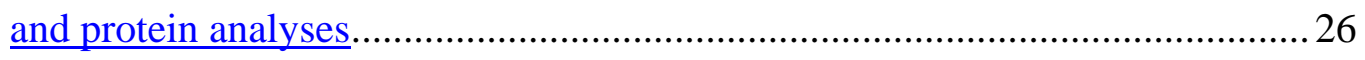

2.1.7 Solutions and chemicals for electrophysiological analyses ................................29

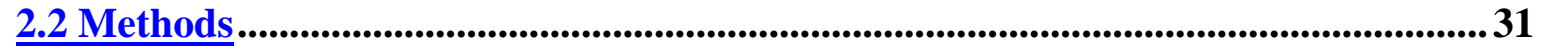

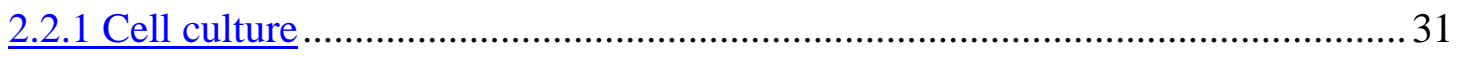

2.2.1.1 Isolation and cultivation of human bone marrow-derived

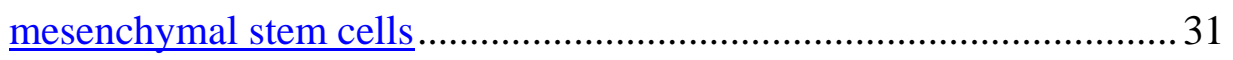

2.2.1.2 Cultivation and inactivation of mouse embryonic fibroblasts................. 31

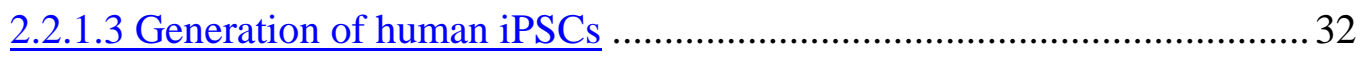

2.2.1.4 Cultivation of human iPSCs and ESCs with feeder cells .........................32

2.2.1.5 Cultivation of human iPSCs without feeder cells..................................... 32

2.2.1.6 Spontaneous differentiation of human iPSCs in vitro ...............................33

2.2.1.7 Directed differentiation of human iPSCs into cardiomyocytes................33

2.2.1.8 Freezing and thawing of cultivated cells ................................................. 34

2.2.2 Alkaline phosphatase staining ........................................................................ 34

2.2.3 Genomic DNA isolation for DNA sequencing ………….................................... 34

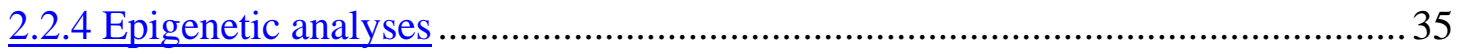




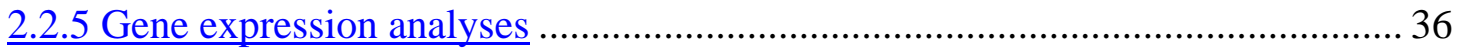

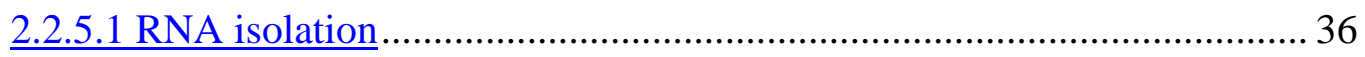

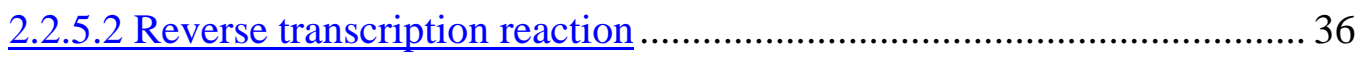

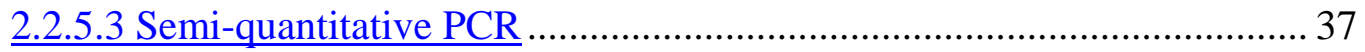

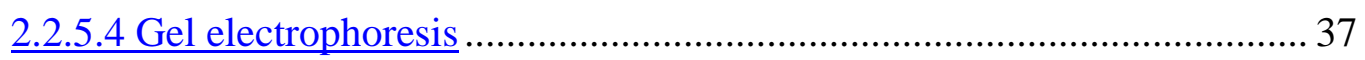

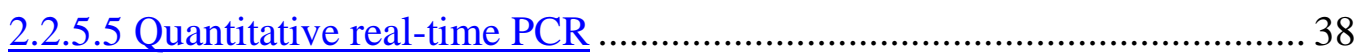

2.2.5.6 Allele-specific expression analysis of the $S C N 5 A$ gene ......................... 39

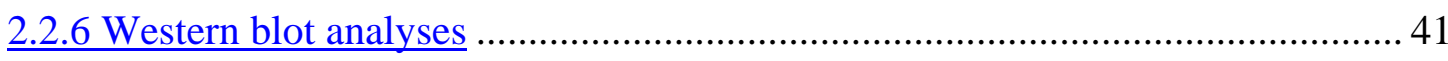

2.2.6.1 Preparation of cell lysates.................................................................... 41

2.2.6.2 SDS-polyacrylamide gel electrophoresis ......................................... 41

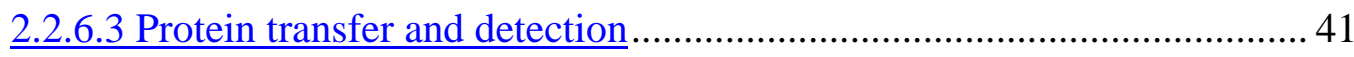

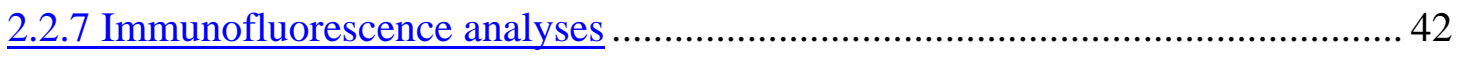

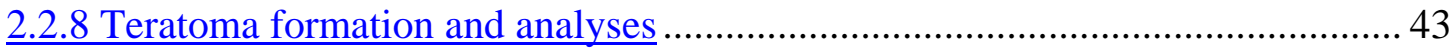

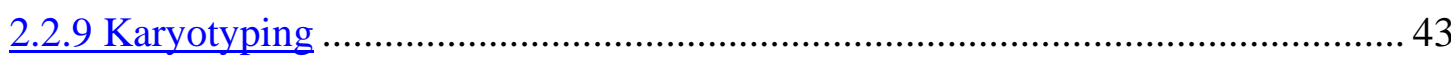

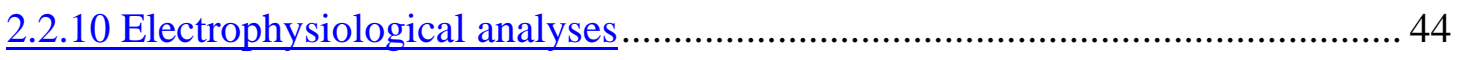

2.2.10.1 Cardiomyocyte preparation and data acquisition .............................. 44

2.2.10.2 Action potential measurements .................................................. 44

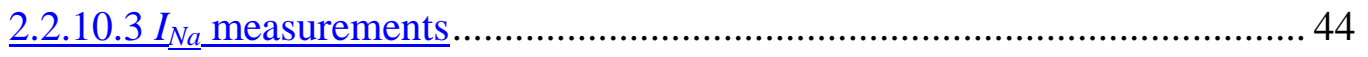

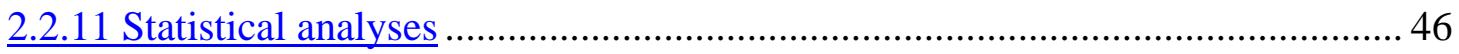

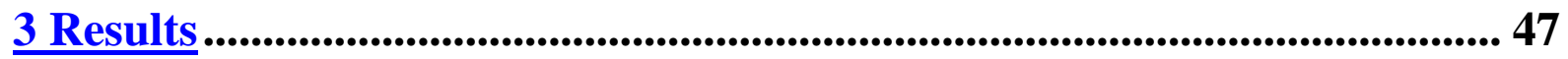

3.1 Clinical profile of a patient suffering from Brugada syndrome ............................... 47

3.2 Generation of human iPSCs and proof of pluripotency............................................... 49

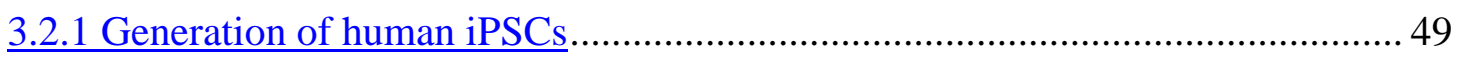


3.2.2 Expression of pluripotency related markers...................................................... 50

3.2.3 Activation of NANOG and OCT4 promoters ..................................................52

3.2.4 Differentiation potential in vitro and in vivo....................................................53

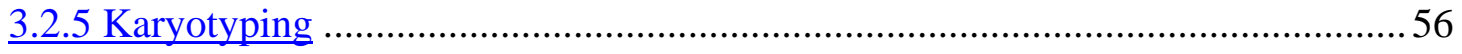

3.2.6 Verification of the SCN5A point mutation in BrS-hiPSCs ……........................57

\subsection{Generation and phenotype characterization of hiPSC-derived}

cardiomyocytes..........................................................................................................................5 58

3.3.1 Ctrl- and BrS-hiPSCs differentiate into cardiomyocytes.....................................58

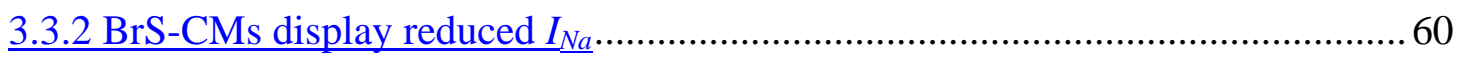

3.3.3 Sodium channels of BrS-CMs show no significant changes in steady-state inactivation, intermediate inactivation, and recovery from inactivation .............63

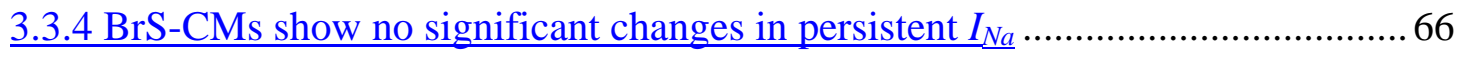

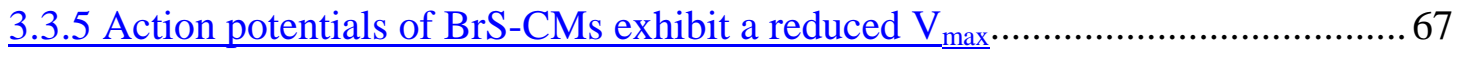

3.3.6 BrS-CMs show increased AP irregularities and beat-to-beat

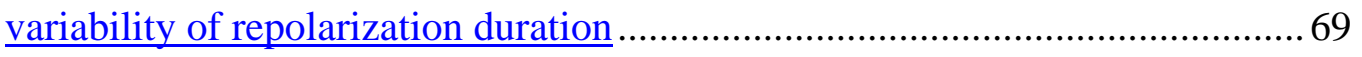

3.3.7 Sodium channel blocker flecainide induces increased APD variability

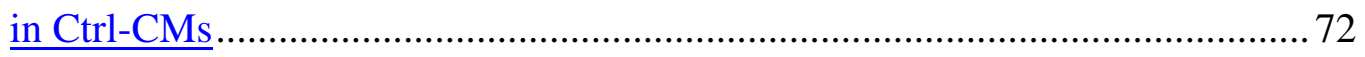

3.3.8 Antiarrhythmic agent quinidine does not decrease APD variability

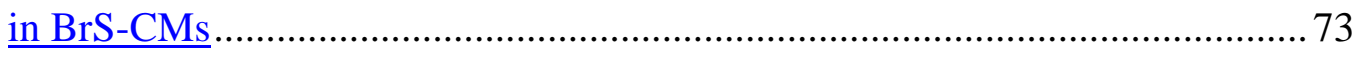

3.4 Role of SCN5A mutation C5435A (p.S1812X) in the pathogenesis of BrS ............. 74

3.4.1 Expression of the SCN5A in BrS-CMs on mRNA level ...................................

3.4.2 Expression of $\mathrm{Na} v 1.5$ protein in BrS-CMs .....................................................

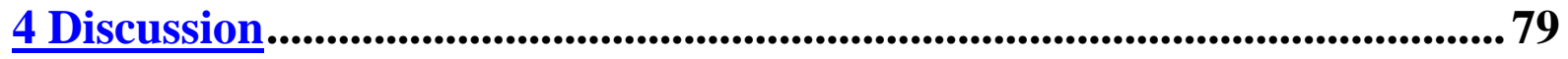

4.1 Successful generation of human iPSCs ...............................................................................8 


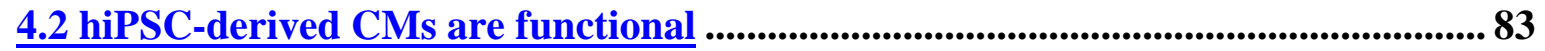

\subsection{BrS-CMs can recapitulate disease-specific physiological phenotypes}

in vitro

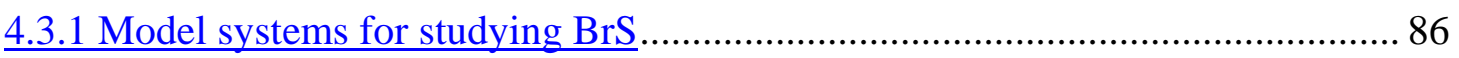

4.3.2 Loss-of-function of cardiac sodium channel in BrS-CMs ................................ 87

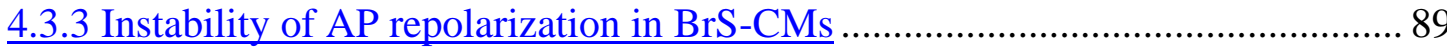

4.4 Molecular mechanisms underlying the loss-of-function in BrS-CMs ....................... 90

4.5 Possible therapeutic treatments of BrS......................................................................... 92

4.5.1 Potential antiarrhythmic drugs in treatment of BrS patients.............................. 92

4.5.2 Readthrough therapy for suppression of nonsense mutation ............................. 94

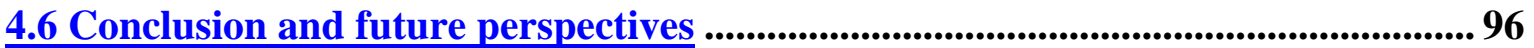

5 Summary ..................................................................................................................... 97

6 References................................................................................................................................... 99

7 Acknowledgements ................................................................................................... 115 


\section{List of abbreviations}

AFP

ALB

$\alpha-\mathrm{MHC}$

AP

APA

APD

APS

ATP

AV

bFGF

bp

$\mathrm{BrS}$

BSA

CamKII

CASQ2

cDNA

cTNT

$\mathrm{C}_{\mathrm{m}}$

CMs

CPVT

Cre

Ctrl

Cy3

Cx43

DAD

DAPI

DEPC

DMEM

DMSO

DPBS

DTT

EAD
Alpha-1-fetoprotein

Albumin

Myosin heavy chain (alpha)

Action potential

Action potential amplitude

Action potential duration

Ammonium persulfate

Adenosine triphosphate

Atrioventricular

Fibroblast growth factor basic

Base pair

Brugada syndrome

Bovine serum albumin

$\mathrm{Ca}^{2+} /$ calmodulin-dependent protein kinase II

Calsequestrin 2

Complementary DNA

Cardiac troponin $\mathrm{T}$

Membrane capacitance

Cardiomyocytes

Catecholaminergic polymorphic ventricular tachycardia

Cyclization recombination

Control

Cyanine 3

Connexin 43

Delayed afterdepolarization

4', 6-diamidino-2-phenylindole dihydrochloride

Diethylpyrocarbonate

Dulbecco's modified Eagle medium

Dimethyl sulfoxide

Dulbecco's phosphate buffered saline

Dithiothreitol

Early afterdepolarization 
EB

ECG

EDTA

EF- $1 \alpha$

EGTA

ESCs

FBS

FITC

FOXD3

GAPDH

GDF3

GTP

HEK

hESCs

hiPSCs

HRP

ICD

$\operatorname{IgG/IgM~}$

IF

IMDM

$I_{C a}$

$I_{C a L}$

$I_{K 1}$

$I_{N a}$

IRES

$I_{\text {to }}$

KLF4

LQTS

MEFs

MLC2a

MOI

MTG

NEAA

NMD
Embryoid body

Electrocardiogram

Ethylenediaminetetraacetic acid

Elongation factor-1 alpha

Ethylene glycol tetraacetic acid

Embryonic stem cells

Fetal bovine serum

Fluorescein isothiocyanate

Forkhead box D3

Glyceraldehyde-3-phosphate dehydrogenase

Growth differentiation factor 3

Guanosine triphosphate

Human embryonic kidney

Human embryonic stem cells

Human induced pluripotent stem cells

Horseradish peroxidase

Implantable cardioverter defibrillator

Immunoglobulin G/M

Immunofluorescence

Iscove's modified Dulbecco's medium

Calcium current

L-type calcium current

Inward rectifier potassium current

Sodium current

Internal ribosome entry site

Transient outward potassium current

Krüppel-like factor 4

Long-QT syndrome

Mouse embryonic fibroblasts

Myosin light chain 2 (atrial)

Multiplicity of infection

Monothioglycerol

Non-essential amino acids

nonsense-mediated mRNA decay 
NMRI

OCT4

PCR

PFA

PTC

RPMI

$\mathrm{R}_{\mathrm{S}}$

RT-PCR

SCN5A

SCNT

SDS-PAGE

SiRNA

SMA

SNP

SOX2

SQTS

SSEA4

STAP

STEMCCA

SYP

TB buffer

TBS-T

TEMED

$\mathrm{TH}$

TMAC

Tris

WB
Naval Medical Research Institute

Octamer binding transcription factor 4

Polymerase chain reaction

Paraformaldehyde

Premature termination codon

Roswell Park Memorial Institute

Series resistance

Reverse transcription PCR

Sodium channel, voltage gated, type V, alpha subunit

Somatic cell nuclear transfer

Sodium dodecylsulfate polyacrylamide gel electrophoresis

Small-interfering RNA

Smooth muscle actin

Single nucleotide polymorphism

SRY-box 2

Short-QT syndrome

Stage-specific embryonic antigen 4

Stimulus-triggered acquisition of pluripotency

Stem cell cassette

Synaptophysin

Tris-borate buffer

Tris-buffered saline with Tween 20

Tetramethylethylenediamine

Tyrosine hydroxylase

Tetramethylammonium chloride

Tris(hydroxymethyl)aminomethane

Western blot 


\section{Introduction}

\subsection{Definition and characteristics of stem cells}

A stem cell is an unspecialized cell, which is capable of renewing itself on the one hand, and giving rise to specialized cells on the other hand. Depending on its biological niche, a stem cell can either divide symmetrically into two identical daughter cells, each with characteristics of a stem cell, or asymmetrically into a stem cell and a more specialized cell, a process called cellular programming or differentiation. This process of differentiation occurs in a series of steps resulting in a cell with an increasingly limited developmental potential. Therefore, stem cells provide the basis for the development of the different tissues and organs of the multicellular organism as well as for its ability to regenerate.

Stem cells are typically classified according to their origin, i. e. embryonic, fetal, neonatal, or adult stem cells, or their developmental potential (Stem Cells: Scientific Progress and Future Research Directions 2001). If a stem cell is capable of giving rise to a whole organism including its extraembryonic tissues, it is regarded to be totipotent. All cells from the (murine) zygote to the 8-cell-morula are totipotent (Kelly 1977). In comparison, pluripotent stem cells can only differentiate into all specialized cell types in the body, encompassing the three embryonic germ layers mesoderm, endoderm, and ectoderm as well as germline cells. Embryonic stem cells (ESCs) represent one famous example for pluripotent stem cells. They are usually derived from the inner cell mass of an early stage embryo, called the blastocyst, before implantation in the uterus would normally occur. Once removed from the blastocyst, these cells can be cultivated in vitro, where they proliferate indefinitely while maintaining an undifferentiated state. Alternatively, they can differentiate into any specialized cell of the more than 200 different cell types of the body. ESCs were first obtained from 4- to 5-day-old mouse embryos (Evans and Kaufman 1981; Martin 1981), but have now been derived from a number of different species including human (Thomson et al. 1998). Multipotent stem cells exhibit an even more restricted developmental potential, being only able to differentiate into specialized cells of several lineages. After embryonic development, adult stem cells reside in various tissues of the fetal, juvenile, or adult body like in the brain, blood vessels or skin epithelia, in skeletal muscles, liver, pancreas and so forth. Here, they replace continuously dying cells and contribute to the regeneration of the tissue from which they originate. Stem cells from the bone marrow are the most studied types of adult stem cells and can be classified into two major types: mesenchymal and hematopoietic stem cells. They are multipotent and 
hence can provide several cell types. Mesenchymal (or stromal) stem cells form bone, cartilage, and fat tissue, whereas hematopoietic stem cells form blood and immune cells. They are already used in clinic for transplantation after cancer treatment to restore the immune system. Unipotent adult stem cells have the capacity to differentiate into one cell type. For example, spermatogonial stem cells can only differentiate into sperm cells, providing a constant supply of sperm cells for an entire lifetime.

\subsection{Cellular reprogramming}

When a sperm and an egg cell fuse together, they form a diploid zygote, which starts to divide continuously to provide enough cells for the generation of a new organism. During embryonic development, most of the initially unspecialized cells develop into somatic cells with characteristic shapes and specialized functions, such as heart muscle cells or neurons. Within this well-controlled process, a differentiating cell gets increasingly restricted in its developmental potential, leading to a cell expressing specific genes for its specific function in the body. The differentiation of cells depends on a stable restriction of their genetic information. Cellular reprogramming on the other hand is a process that converts one specific cell type into another, including the induction of a somatic cell into a pluripotent stem cell (Nicholas and Kriegstein 2010). As mentioned above, cells in a pluripotent state can turn into the various types of cells that make up the body. Therefore, they are ideal for studying the development of diseases or the effectiveness of drugs, and could also be transplanted to regenerate failing organs, which is described in a later chapter.

\subsubsection{Inducing pluripotency}

In the last 60 years, researchers put much effort in uncovering the mechanism underlying cellular reprogramming. The first study was performed in amphibians at the beginning of the second half of the $20^{\text {th }}$ century. In the early 1950's, Robert Briggs and Thomas King invented a technology, called somatic cell nuclear transfer (SCNT), where they transplanted nuclei from different embryonic developmental stages of the frog Rana pipiens into enucleated and activated oocytes (Briggs and King 1952). Based on these experiments, Sir John B. Gurdon was able to show that even more differentiated nuclei from the tadpoles' intestinal epithelium of Xenopus laevis could be reprogrammed to a totipotent state after transplantation into enucleated oocytes (Gurdon 1962). Some of these oocytes fertilized in this way were able to develop into new tadpoles. The efficiency of the nuclear reprogramming could even be 
improved by serial transplantations, i. e. taking the nuclei from the developed blastulae and transplanting them again into enucleated and activated oocytes.

It took a further 35 years until the group of Sir Ian Wilmut demonstrated that not only amphibian, but also mammalian eggs have the potential to convert a somatic state of a nucleus back to the totipotent state (Wilmut et al. 1997). By using the SCNT method, Wilmut and his colleagues were able to reprogram the nucleus of an adult mammary epithelial cell, resulting in the first cloned mammal, Dolly the sheep. Since Dolly, many other mammalian species had been cloned (Wakayama and Yanagimachi 1999; Grisham 2000; Hochedlinger and Jaenisch 2002).

Furthermore, ESCs were known to induce pluripotency in somatic cell nuclei after induced cell fusion of both cell types (Tada et al. 2001). All these experiments revealed that differentiated cell nuclei can be reverted into an undifferentiated state with a higher developmental potential. This reversion must have been induced by unknown factors contained in oocytes and ESCs. Both methods for inducing toti-/pluripotency have several disadvantages. The SCNT method is technically challenging and requires huge sources for oocytes. The cell fusion method is also inefficient, and in addition, generates tetraploid cells.

\subsubsection{Induced pluripotent stem cells}

It was not until 2006, when the factors being crucial for nuclear reprogramming were identified (Takahashi and Yamanaka 2006). In their studies, Takahashi and Yamanaka focused on factors which are important for the maintenance of pluripotency in ESCs. They initially selected a set of 24 transcription factors and introduced them into murine skin fibroblasts by retroviral transfection. Some of these cells formed colonies that showed similar characteristics to ESCs. By excluding a series of factors in a stepwise manner, Takahashi and Yamanaka finally identified a combination of only four transcription factors, encoded by Oct4 (octamer binding transcription factor 4), Sox2 (SRY-box 2), Klf4 (Krüppel-like factor 4), and $c-M y c$ that was sufficient to induce pluripotency by overexpression in somatic cells. They called these cells induced pluripotent stem cells (iPSCs). However, unlike ESCs, the generated iPSCs from the first study failed in germ line transmission through chimeric mice. This problem was solved in the second iPSC generation (Okita et al. 2007; Maherali et al. 2007; Wernig et al. 2007). The first human iPSCs (hiPSCs) could be generated in the same year by using the same factors (Takahashi et al. 2007). Yu and colleagues from James A. 
Thomson's lab used a different combination of genes for the reprogramming (Yu et al. 2007). Here, they successfully introduced OCT4, SOX2, NANOG, and LIN28 into human fibroblasts, avoiding the transduction with the proto-oncogene $c-M Y C$.

For their fundamental discovery, Yamanaka together with Sir John B. Gurdon were awarded with the Nobel Prize in Physiology or Medicine 2012 "for the discovery that mature cells can be reprogrammed to become pluripotent" (The Nobel Prize in Physiology or Medicine 2012, press release 2012). The iPSC technology opened a new, incredibly fast growing research field, which has been adopted by many laboratories all over the world.

Substantial progress has been made since the first generated iPSCs in 2006. For instance, besides fibroblasts, iPSCs could be generated from many other cell types (Aoi et al. 2008; Aasen et al. 2008; Haase et al. 2009; Novak et al. 2010; Chen et al. 2013). It also appeared that in some cell types, fewer than four factors are sufficient to induce pluripotency, as shown in adult mouse neural stem cells, where only Oct4 is needed (Kim et al. 2009). Researchers have also rapidly improved the techniques to generate integration-free iPSCs. First iPSCs were generated by random genomic integration of retroviruses containing the four Yamanaka factors (Takahashi and Yamanaka 2006). Genomic integration carries the risk of destroying functional genes or activating oncogenes, which might result in cancer cells (Okita et al. 2007). Currently, it is already possible to generate iPSCs with nonintegrating viral vectors, like adenoviruses (Stadtfeld et al. 2008) or plasmids (Okita et al. 2008). The attempt to reprogram somatic cells even with a DNA-free method has also successfully been shown by introducing the reprogramming factors in the form of recombinant proteins into somatic cells (Zhou et al. 2009; Kim et al. 2009). However, the recombinant proteins are challenging to be produced and purified in the required quantities. In addition, the reprogramming efficiency to generate protein-induced pluripotent stem cells is still relatively low. Other approaches to circumvent the reprogramming with DNA is to use RNA as a vehicle, like the Sendai virus, which has a completely RNA-based reproductive cycle (Fusaki et al. 2009; Ye et al. 2013), or synthetically modified mRNA as transcripts of the four Yamanaka factors generating RNAinduced pluripotent stem cells (Warren et al. 2010).

\subsubsection{Stimulus-triggered pluripotency}

Very recent studies indicate that some somatic cells might possess surprisingly huge plasticity when exposed to strong external stimuli. Obokata and colleagues showed that leukocyte- 
specific antigen CD45 positive hematopoietic cells could obtain pluripotency after low-pH treatment (Obokata et al. 2014a, b). This reprogramming phenomenon does not require the introduction of any transcription factor into the cell and is called "stimulus-triggered acquisition of pluripotency (STAP)". Other types of sublethal stress, such as physical damage, plasma membrane perforation, heat shocks or high calcium exposure, might also lead to reprogramming events in somatic cells, leaving open questions that still need to be addressed. Currently, many scientists who tried to reproduce STAP cells have failed, leading to an investigation of Obokata's publications by the RIKEN research institute (http://www.riken.jp/en/pr/press/2014/20140314_1). However, if their results are proven to be valid, STAP cells could represent an innovative, easy, and efficient way to generate pluripotent stem cells and might open a new area in stem cell biology.

\subsubsection{Direct transdifferentiation}

Yamanaka's approach to systematically define a small set of transcription factors to bring a somatic cell back to an embryonic stem cell-like state has inspired many scientists to find a way in changing the fate of a cell without passing through the pluripotent state, a process called direct transdifferentiation or direct reprogramming. Findings in an early study showed that a single factor, MyoD, was able to transdifferentiate mouse fibroblasts into skeletal muscle cells (Davis et al. 1987). However, attempts to identify a single "master" transcription factor have failed until then for most somatic lineages. In light of the success of the iPSC technology, fibroblasts could be directly converted into neuronal-, hepatocyte-, or cardiomyocyte (CM)-like cells using a combinatorial delivery of multiple transcription factors or microRNAs (Vierbuchen et al. 2010; Ieda et al. 2010; Huang et al. 2011).

All these efforts in improving cellular reprogramming by trying to overcome genomic manipulation and at the same time to increase the reprogramming efficiency, seek to make these cells safer and therefore applicable for a potential clinical use.

\subsection{Cardiac electrophysiology and arrhythmias}

\subsubsection{The cardiac conduction system}

The human heart is the first functional organ in the developing embryo, showing its importance in providing each cell of the body with oxygen and nutrients by persistent beating throughout life (Developmental Biology, $9^{\text {th }}$ Edition, Sinauer Associates Inc.). During systole, 
blood is pumped out of the contracting heart chambers followed by diastole when the heart chambers relax and refill with blood. Although the heart is enervated by the autonomic nervous system, it is capable to beat autorhythmically, initiated by specialized cardiac cells in the sinoatrial node, located right next to the superior vena cava in the right atrium. Like all CMs, they are electrically excitable cells, capable of initiating and propagating action potentials (APs). These cells control the heart rate and are therefore called pacemaker cells. The generated electrical impulses spread throughout both atria, resulting in their contraction, and then toward the atrioventricular (AV) node which is located in the septum between the right atrium and ventricle. The electrical conduction is delayed for about $0.1 \mathrm{~s}$ to ensure that the blood is pumped out of both atria into the ventricles completely, before the latter start to contract. The electrical signals propagate further through the conduction system, composed of the bundle of His and the Purkinje fibers, to the apex of the heart, where it spreads throughout the ventricular myocardium. The electrical activity of the heart over time can be detected by electrodes and visualized with an electrocardiogram or ECG (Fig. 1).

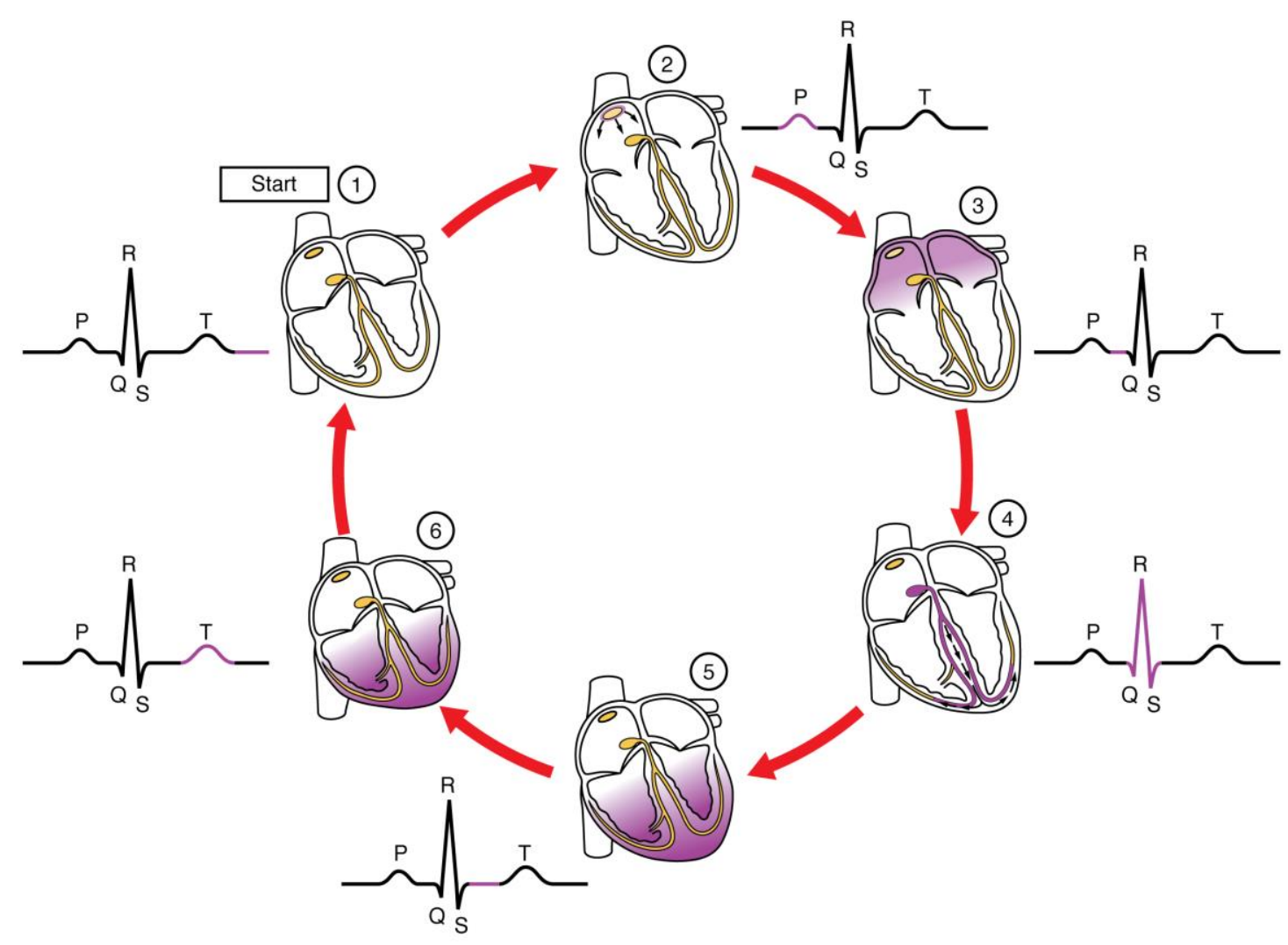

Figure 1. Correlation of an ECG tracing with the electrical events in the heart. (1) Cells of sinoatrial node are at rest. Cells of the sinoatrial node initiate electrical signals spreading throughout the atria represented by a prominent P-wave on ECG (2). During propagation of the electrical signals towards the AV node, the atria start to contract (3). The electrical signals propagate further through the conduction system to the apex of the heart (4) followed by ventricular depolarization (5) and contraction (6). The QRS-complex on the ECG represents the ventricular depolarization. The ventricular repolarization is illustrated by a T-wave. (Figure taken from CNX Anatomy and Physiology: http://cnx.org/content/m46664/latest/?collection=col11496/latest) 
The ECG is used as an indispensable clinical tool to monitor the state of heart function. In a standard surface ECG, the cardiac cycle is illustrated in typical P-QRS-T-waveforms. The Pwave represents the depolarization of the atria, followed by their contraction. The QRScomplex represents the ventricular depolarization. The ventricles begin to contract at the peak of the R-wave. Finally, the T-wave depicts the repolarization, which means the electrical recovery of the ventricles. The different segments and intervals of an ECG trace, like the PRsegment or the QT-interval, can give important clinical information about the condition of a patient's heart.

\subsubsection{Action potentials and excitation-contraction coupling}

In a healthy heart, the generated electrical signals of the sinoatrial node are propagated by the CMs in a strongly synchronized way leading to simultaneous contractions of the cells. The process from the electrical excitation of the myocytes to a contraction of the heart is called "excitation-contraction coupling". To ensure a fast electrical conduction, CMs are tightly connected to each other through intercalated discs, composed of desmosomes and connexones. Thus, small molecules and ions can pass from one cell to another rapidly. The heart consists of two major types of cardiac muscle cells: myocardial conducting and contractile cells.

The myocardial conducting cells form the conduction system of the heart. They initiate and propagate APs at a constant rate, without possessing a stable resting membrane potential (RMP). Rather, their membrane potential is always drifting towards a threshold, caused by a slow influx of $\mathrm{Na}^{+}$ions into the cell. This drift is called the pacemaker potential. Upon reaching this threshold, calcium channels open and the rapid influx of $\mathrm{Ca}^{2+}$ ions leads to a depolarization of the cell. The calcium channels close at the maximum of the depolarization rate. At the same time, potassium channels open, allowing the efflux of $\mathrm{K}^{+}$ions and resulting in repolarization of the cell.

In contrast, the APs of the myocardial contractile cells, which form the main part of the atria and ventricles, can be subdivided into five phases (Fig. 2). In the resting state (phase 4), adult CMs have a very negative membrane potential at approximately -80 to $-90 \mathrm{mV}$. It is caused by different ion concentrations across the membrane and its selective permeability toward various ions. The RMP is dominated by the potassium equilibrium potential according to its 
electrochemical gradient across the cell membrane. Inward rectifying potassium channels, as well as the $\mathrm{Na}^{+} / \mathrm{K}^{+}$and $\mathrm{Na}^{+} / \mathrm{Ca}^{2+}$ ion pumps contribute to its maintenance.

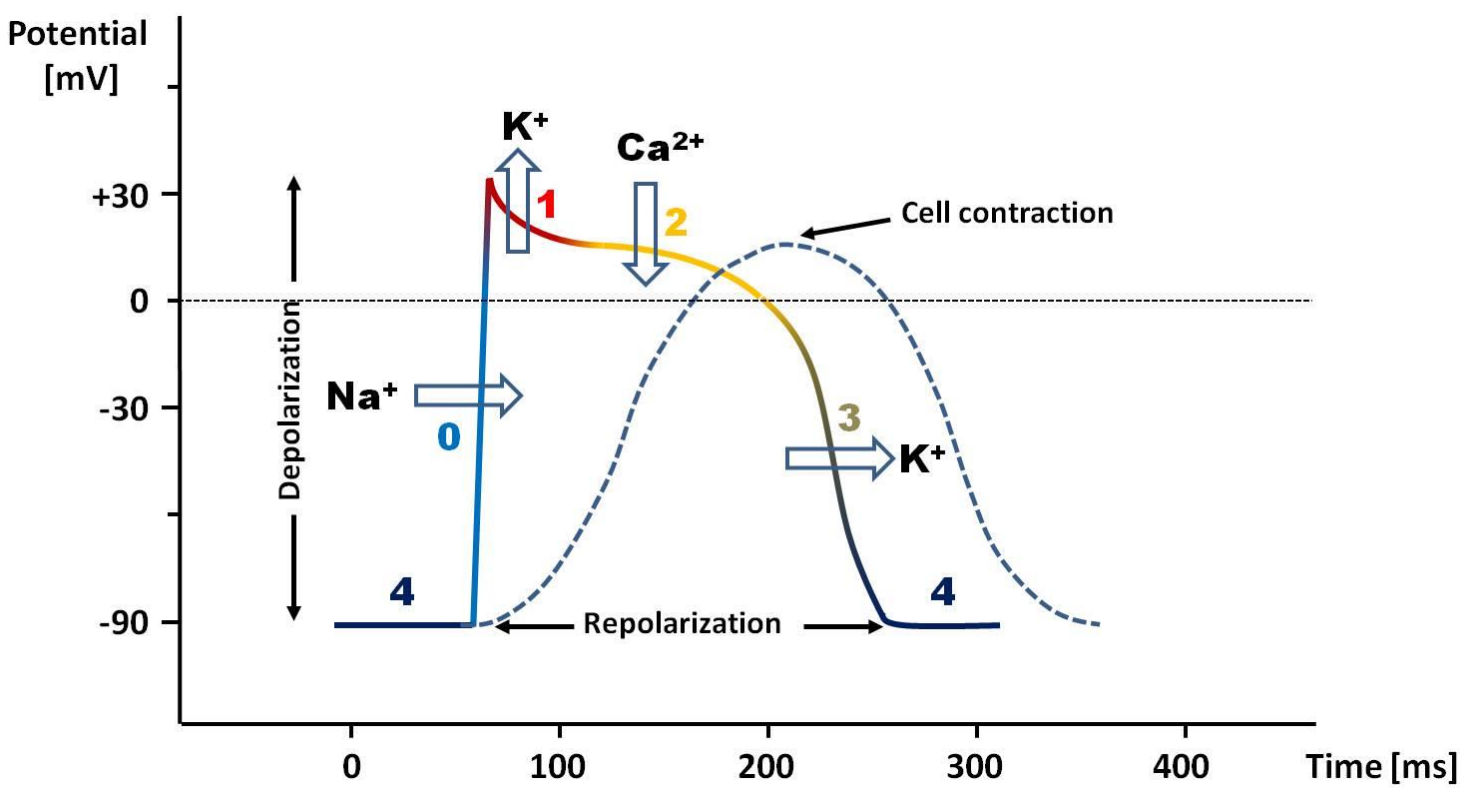

Figure 2. AP of myocardial contractile cells. A typical ventricular AP can be subdivided into five phases. The RMP of approximately $-90 \mathrm{mV}$ is caused by the difference in ionic concentrations and conductances across the membrane and is dominated by the potassium equilibrium potential. If the cell gets stimulated by an adjacent cell, sodium channels open and a rapid influx of $\mathrm{Na}^{+}$ions occurs, leading to the depolarization of the cell (phase 0 ). Phase 1 is characterized by an early repolarization ("notch") due to the inactivation of the fast sodium channels and a net efflux of $\mathrm{K}^{+}$ions. The following plateau phase is sustained by a balance between the net inward calcium and outward potassium currents. The $\mathrm{Ca}^{2+}$ ion influx leads to cell contraction. Phase 3 is predominated by a net $\mathrm{K}^{+}$ion efflux, leading to the repolarization of the cell. The delayed rectifier potassium channels close, when the membrane potential is restored.

In phase 0 , voltage-gated sodium channels located in the plasma membrane open immediately after electrical stimulation coming from an adjacent cell. This leads to a rapid influx of $\mathrm{Na}^{+}$ ions into the cell and causes the depolarization of the cell. Phase 1 of the AP is due to the immediate inactivation of the fast sodium channels and the simultaneous transient outward current of $\mathrm{K}^{+}$ions $\left(I_{t o}\right)$. This short repolarization is followed by a plateau phase, where $\mathrm{Ca}^{2+}$ ions enter the cell through voltage-gated L-type calcium channels (phase 2). The plateau is caused by a balance between the inward calcium current and the outward potassium current. After the inactivation of the calcium channels, the cell repolarizes rapidly as the slow delayed rectifier potassium channels remain open, accompanied by the additional opening of rapid delayed rectifier potassium channels as well as inward rectifying potassium channels (phase 3). The delayed rectifier potassium channels close, when the RMP is accomplished and the cycle starts again. 
The extended refractory period during the plateau phase allows the cell to fully contract before another AP can occur. During this phase, $\mathrm{Ca}^{2+}$ ions enter the cell and activate ryanodine receptors resulting in the $\mathrm{Ca}^{2+}$ ion release from the sarcoplasmic reticulum into the cytosol. This positive feedback process is called "calcium-induced calcium release" and leads to a significantly increased cytoplasmic $\mathrm{Ca}^{2+}$ ion concentration, which is essential for the cell contraction. The released $\mathrm{Ca}^{2+}$ ions bind directly to the protein troponin $\mathrm{C}$, which is part of the sarcomeric myofilaments and switches on the contractile machinery. For relaxation of the cell, intracellular $\mathrm{Ca}^{2+}$ ions must decline, allowing $\mathrm{Ca}^{2+}$ ions to dissociate from troponin. This requires $\mathrm{Ca}^{2+}$ ion transport out of the cytosol by four pathways involving sarcoplasmic reticulum $\mathrm{Ca}^{2+}$-ATPase, sarcolemmal $\mathrm{Na}^{+} / \mathrm{Ca}^{2+}$ exchange, sarcolemmal $\mathrm{Ca}^{2+}$-ATPase or mitochondrial $\mathrm{Ca}^{2+}$ uniport (for review see Bers 2002).

\subsubsection{Structure, function, and regulation of the cardiac sodium channel}

The cell membrane consists of a hydrophobic phospholipid bilayer, which forms a natural barrier to ions and other molecules. Ion pumps and ion channels ensure a controlled exchange of ions between the inside of a cell and its environment. Ion channels are integral transmembrane proteins and are highly selectively permeable to certain ions. They can change between an opened and a closed state, a process, which is called gating. The gating process in different ion channels may be triggered by a voltage change across the cell membrane, by binding of ligands to the channel, or by the phosphorylation status of the channel as well as mechanical or other stimuli. In CMs, various ion channels contribute to the maintenance of the negative RMP and the generation of APs, which finally leads to the contraction of the cell (Fig. 2).

The cardiac sodium channel consists of a transmembrane pore-forming $\alpha$-subunit associated with one or two ancillary modulatory $\beta$-subunits (Abriel 2010). It is responsible for the rapid upstroke during phase 0 of nonpacemaker APs through a fast inward sodium current $\left(I_{N a}\right)$ and for the maintenance of the electrical conduction in the heart. The family of voltage-gated sodium channel $\alpha$-subunits encompasses nine members $\left(\mathrm{Na}_{\vee} 1.1-\mathrm{Na}_{\vee} 1.9\right)$ with a homology of more than $50 \%$ in their amino acid sequences (Catterall et al. 2005). The isoform $\mathrm{Na}_{\mathrm{V}} 1.5$ is predominantly, but not exclusively expressed in the human heart (Blechschmidt et al. 2008). The 2016 amino acids long $\alpha$-subunit is encoded by the sodium channel, voltage-gated, type $V$, alpha subunit (SCN5A) gene, which is located on chromosome $3 \mathrm{p} 21$ and consists of 28 exons. Immunocytochemical staining in murine CMs showed its preferential localization 
in intercalated discs (Maier et al. 2002; Maier et al. 2004) and in the T-tubular system. Nav1.5 is composed of four homologous domains (DI - DIV), each containing six transmembrane segments (S1 - S6). The four domains are connected to each other by cytoplasmic linker sequences (see Fig. 4). The positive charges of amino acid residues in the S4 segments are assumed to act as the voltage sensor important for activating the channel, whereas the linking region between DIII and DIV together with the $\mathrm{COOH}$ terminus is thought to act as the inactivation gate (Stühmer et al. 1989; West et al. 1992; Motoike et al. 2004). The region between S5 and S6 within the four domains forms the narrowest part of the pore and the negatively charged amino acid residues are responsible for ion selectivity.

$\mathrm{Na}_{\mathrm{V}} 1.5$ is a dynamic molecule which changes its structural conformation in response to voltage changes across the cell membrane. It can exist in an activated (opened), inactivated (closed), or deactivated (closed) state (Fig. 3).

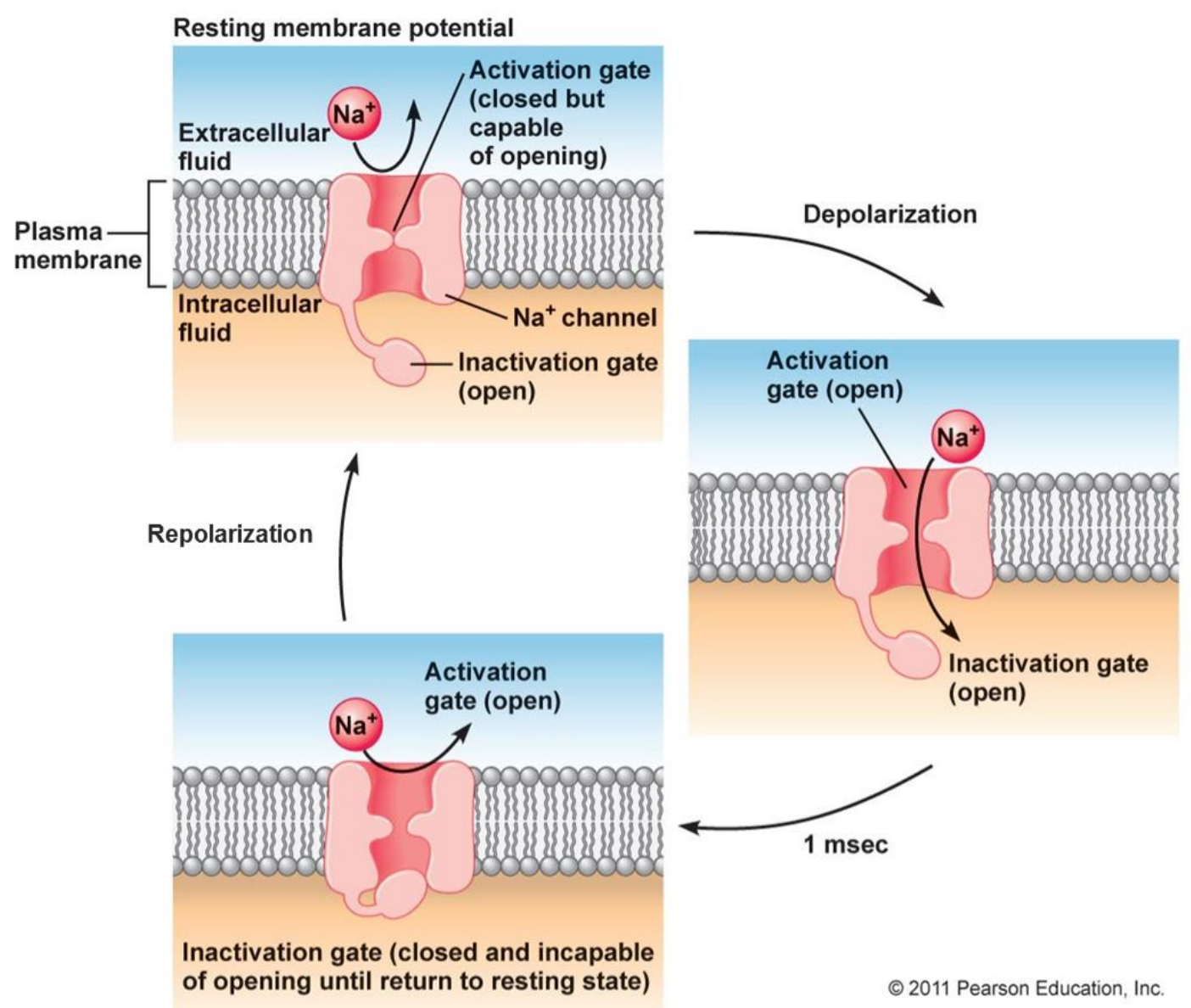

Figure 3. The conformational states of the voltage-gated sodium channel. During RMP, the activation gate of the sodium channel is closed, preventing $\mathrm{Na}^{+}$ions from entering the cell (resting or deactivated state). Upon depolarization, the activation gate opens, allowing $\mathrm{Na}^{+}$ions to pass the membrane (activated state). When the inactivation gate closes, the inward $I_{N a}$ is blocked (inactivated state). During repolarization the sodium channel returns to its deactivated state. (Figure modified and taken from: http://droualb.faculty.mjc.edu/Course $\% 20$ Materials/Physiology\%20101/Chapter\%20Notes/Fall\%202011/chapter_7\%20Fall\%202011.htm) 
According to this model, the activation gate of the sodium channel is closed in the resting state of the cell, whereas the inactivation gate is open. Upon voltage changes of the membrane coming from adjacent cells, the fast opening of the activation gate in the voltage-sensitive channel allows $\mathrm{Na}^{+}$ions to enter and therefore depolarize the cell immediately. After a few milliseconds, the inactivation gate closes and stops the inward current. The channel is inactivated and not able to open again until further conformational changes. During repolarization, the activation gate closes and the inactivation gate reopens. The channel is now again in its deactivated state and ready to open with the next voltage change of the membrane.

Although $\mathrm{Na}_{v} 1.5$ is already a self-forming functional unit that conducts $\mathrm{Na}^{+}$ions, a large number of proteins are involved in the regulation of the cardiac sodium channel (reviewed by Abriel 2010). The $\beta$-subunits directly interact with the $\alpha$-subunit through their extracellular immunoglobulin-fold domains and play important roles in Nav1.5 cell surface expression and channel gating. Four $\beta$-subunits $(\beta 1-\beta 4)$ have been identified in the human heart. They all consist of an $\mathrm{N}$-terminal domain, a transmembrane segment, and an intracellular $\mathrm{C}$-terminal domain. In addition, several other proteins, including adapter, accessory, cytoskeletal and regulatory proteins are associated with $\mathrm{Na}_{\mathrm{v}} 1.5$, forming macromolecular complexes and are involved in regulation of the channel activity, trafficking, and cellular localization as well as channel biosynthesis and degradation (for more details see Abriel 2010 or Rook et al. 2012).

\subsubsection{Cardiac sodium channelopathies}

Cardiac arrhythmias are any inotropic or chronotropic alterations in the rhythm of the heart beat. Thereby, the electrical activity of the heart can either be too slow (bradycardia) or too fast (tachycardia) in a regular or irregular way. Both the atria as well as the ventricles can be affected. Cardiac arrhythmias can be life-threatening and are one of the major causes of mortality in developed countries (Wolf and Berul 2008). Sudden arrhythmic deaths can either be associated with a structural heart disease or with an electrical disease in the structurally normal heart. Many arrhythmic syndromes are now known to have an underlying genetic background. Arrhythmias primarily caused by mutations in genes encoding for cardiac ion channels, including sodium, potassium and calcium channels, are called cardiac channelopathies (Wilde and Bezzina 2005). The first mutation in SCN5A was reported in patients with Long-QT syndrome (LQTS) type 3 (Wang et al. 1995). In 1998, mutations in SCN5A were also described in patients with Brugada syndrome (BrS, Chen et al. 1998). To 
date, more than 200 different mutations in the SCN5A gene have been identified, the vast majority in patients with either LQTS type 3 or BrS (Ruan et al. 2009; Fig. 4).

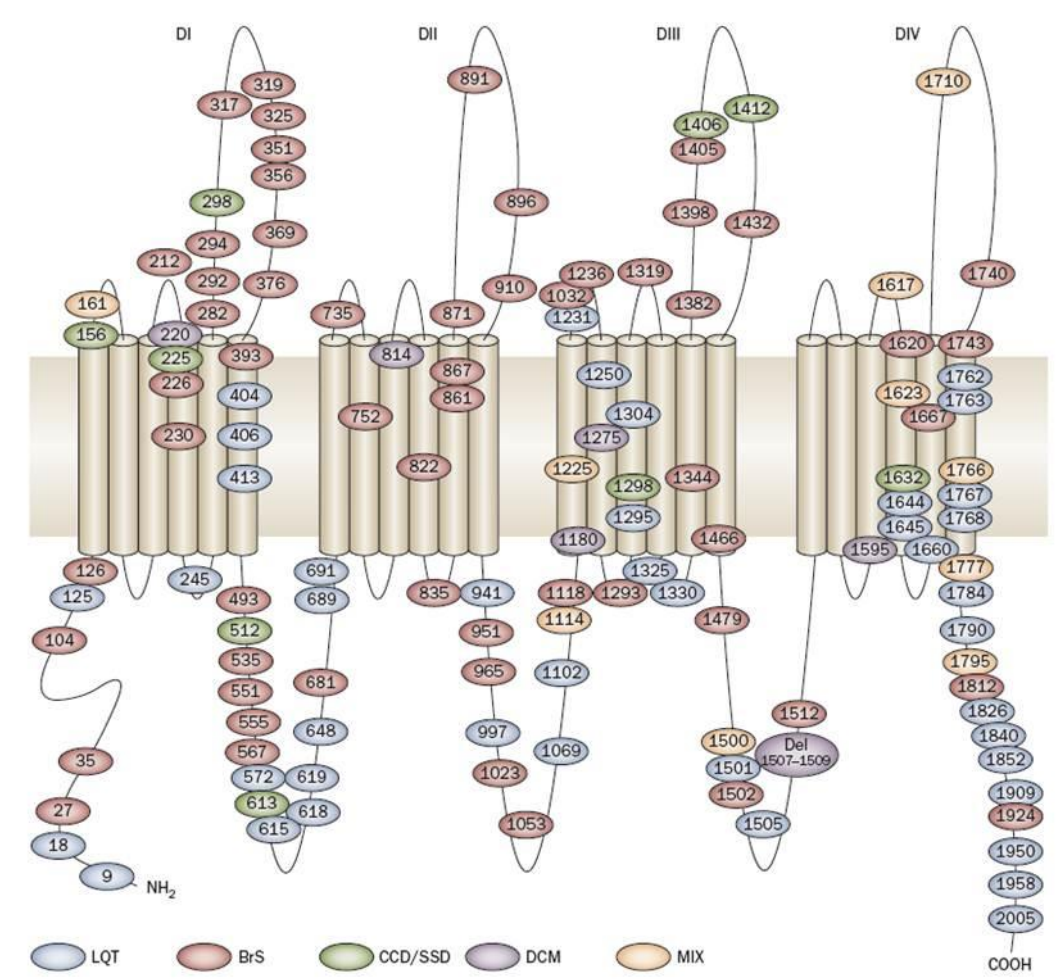

Figure 4. Localization of different mutations in the $\alpha$-subunit of the cardiac sodium channel associated with arrhythmogenic cardiac disorders. LQT: Long-QT syndrome, BrS: Brugada syndrome, CCD: cardiac conduction disease, SSD: sick sinus node syndrome, DCM: dilated cardiomyopathy, MIX: mixed phenotype. (Figure taken from Ruan et al. 2009)

Several other cardiac diseases are also linked to mutations in this gene, such as cardiac conduction disease, sick sinus node syndrome, and dilated cardiomyopathy. Besides these distinct diseases, some genetic defects lead to overlapping syndromes, where clinical characteristics of more than one disease exist in the same patient. The broad phenotypic range of SCN5A mutations indicates the importance of tight sodium channel regulation in maintaining normal cardiac rhythmicity. Mutations at different sites of the protein might cause distinct conformational changes leading to specific electrophysiological alterations. This may provide an explanation why different mutations in the SCN5A gene can lead to either loss- or gain-of-functions of the protein, and therefore to different clinical phenotypes. In addition, mutations in the genes encoding for the $\beta$-subunit of the cardiac sodium channel as well as for the proteins in the associated macromolecular complex can also cause arrhythmias (Ruan et al. 2009). 


\subsubsection{SCN5A mutations and Long-QT syndrome}

LQTS is a hereditary cardiac disease characterized by prolonged QT-intervals on the ECG and a high risk of life-threatening arrhythmias. Mutations in several genes (KCNQ1, KCNH2, $K C N E 1, K C N E 2, C A C N A 1 c, C A V 3, S C N 5 A$, and SCN4B) can cause LQTS. Various subtypes of LQTS exist, each associated with distinct clinical features and underlying genetic defects. LQTS can be inherited in an autosomal dominant or an autosomal recessive fashion. The most common causes are mutations in the genes $K C N Q 1$, KCNH2, and SCN5A, leading to LQTS type 1,2 , and 3 , respectively.

LQTS type 3 is characterized by an abnormal prolongation of the ventricular repolarization revealed by a prolonged QT interval on the ECG with susceptibility to ventricular tachycardia and ventricular fibrillation. Patients with LQTS type 3 display arrhythmias mainly at slow heart rates (e. g. during rest or sleep), and are often present with bradycardia (Schwartz et al. 2001). Cardiac arrest is often the first clinical event and patients are at high risk for sudden death (Zareba et al. 2001).

A large number of $S C N 5 A$ mutations have been characterized as leading to or predisposing to LQTS type 3. The SCN5A mutations involved in LQTS type 3 slow the inactivation of the sodium channel, resulting in prolongation of the $\mathrm{Na}^{+}$ion influx during depolarization. In addition, the mutant sodium channels recover faster from inactivation, allowing for sodium channels to reopen, leading to an increased persistent $I_{N a}$ during the AP plateau phase. Therefore, delayed repolarization and prolonged action potential durations (APDs) occur, and subsequent early afterdepolarizations (EADs) may trigger torsades de pointes and sudden cardiac death (Bennett et al. 1995).

\subsubsection{SCN5A mutations and Brugada syndrome}

The $\mathrm{BrS}$ is a congenital cardiac disease which may cause unexpected sudden cardiac death in apparently healthy people due to severe disturbances of the heart rhythm. BrS usually affects young and middle-aged males in their third or fourth decade of life but can also appear less frequently during infancy (Antzelevitch and Fish 2006; Chen and Priori 2008). The symptoms typically occur during sleep or at rest, and may include seizures, syncope, arrhythmias as polymorphic ventricular tachycardia or ventricular fibrillation and even cardiac arrest (Antzelevitch et al. 2005). The disease was first recognized by the Brugada brothers as a new clinical entity in the early 1990's (Brugada and Brugada 1992). Patients diagnosed with BrS 
have a structurally normal heart but show an abnormal ECG, characterized by an atypical right bundle branch block and a ST-segment elevation in the precordial leads $V_{1}$ to $V_{3}$ of a classical 12-lead ECG. The ECG manifestations of BrS patients are often dynamic and can vary spontaneously, i. e. it can be abnormal at times, but completely normal at others. For diagnostic purposes, the typical ECG pattern in BrS patients can be provoked by the administration of the sodium channel blockers ajmaline, flecainide, or procainamide to confirm or demask BrS (Antzelevitch and Fish 2006).

The only available and effective treatment for $\mathrm{BrS}$ patients today is an automatic implantable cardioverter-defibrillator (ICD; Brugada et al. 1999; Antzelevitch and Fish 2006). It senses the heart beats and gives an electrical shock, when potentially dangerous ventricular arrhythmia is detected. These electrical shocks are painful to the patient, but prevent him from sudden cardiac death.

$\mathrm{BrS}$ is inherited autosomal dominant but with incomplete penetrance. Mutations in the genes encoding for the $\alpha$ - and $\beta$-subunits of the cardiac L-type calcium channel (CACNA1C, $C A C N B 2 b)$, the $\beta$-subunits of the sodium channel (SCN1B, SCN3B), glycerol-3phosphatedehydrogenase 1-like enzyme (GPDIL), as well as genes that effect the potassium outward current (KCNE3, KCND3, KCNE5) are linked to BrS (reviewed by Mizusawa and Wilde 2012). In about $18-30 \%$ of all BrS patients, a mutation in the SCN5A gene was identified (Antzelevitch et al. 2005). Today more than 100 different SCN5A mutations have been reported, including missense mutations, nonsense mutations, splice site mutations, and nucleotide insertions/deletions, which may alter mRNA splicing or create a stop codon by shifting the open reading frame (Mizusawa and Wilde 2012). Although extensive research on $\mathrm{BrS}$ has revealed parts of its genetic background and clinical characteristics in the last two decades, many questions still remain. For example, the mechanism that plays the central role of the disease is not clearly understood. Furthermore, limited progress has been made in the development of treatment strategies.

\subsubsection{Model systems for studying SCN5A mutation-caused channelopathies}

The majority of current functional studies investigating the underlying electrophysiological consequences of SCN5A mutations have either relied on heterologous expression systems or on transgenic mouse models. 
In heterologous expression systems, the mutated gene of interest is ectopically expressed in noncardiac cells, such as Xenopus oocytes, human embryonic kidney (HEK) cells or Chinese hamster ovary cells (Wei et al. 1999; Bankston et al. 2007; Makita et al. 2008). These model sytems do not consider the macromolecular protein complex, in which ion channels are naturally embedded. However, this might be essential to reproduce the electrophysiological and molecular phenotype of the mutation. In this respect, characterization of sodium channel mutations using heterologous expression systems in nonexcitable cells can produce inconsistent and even confounding results. For instance, Watanabe and colleagues showed that heterologous expression of SCN5A mutation G3823A (p.D1275N) in Chinese hamster ovary cells or tsA201 demonstrated near-normal sodium channel function. In contrast, the same mutation expressed in knock-in mice revealed impaired sodium channel function, faithfully phenocopying the human disease (Watanabe et al. 2011).

Several transgenic mouse models carrying SCN5A mutations have been established (reviewed by Derangeon et al. 2012). The first knock-in mouse model $\left(S C N 5 A^{\Delta /+}\right)$ containing a three amino acid deletion at position 1505 - 1507 ( $\Delta \mathrm{KPQ}$ ) was generated for studying LQTS type 3 (Nuyens et al. 2001). This mouse model showed typical features of LQTS type 3, such as QT prolongation on ECG, spontaneous ventricular tachycardia and EADs caused by increased persistent $I_{N a}$. In addition, an early study reported that knock-in mice carrying the $S c n 5 a$ mutation (p.1798insD) revealed overlap features of both LQTS type 3 and BrS (Remme et al. 2006), similar to the phenotype of patients carrying the human equivalent mutation 5387 5389insTGA (p.1795insD; Bezzina et al. 1999).

In contrast to the heterologous expression systems, mouse models provide important constituents of the complex living environment of an ion channel so as to reproduce the exact molecular and electrophysiological phenotype. Mouse models of sodium channelopathies appear as promising tools for understanding the pathophysiological sequence of the diseases. However, there are some limitations of the mouse models, which are mainly associated with the differences between mouse and human physiology, such as the heart rate (much higher rate in mice) and AP morphology (no plateau phase and a shorter APD in mice), as mice use less L-type calcium channels. In addition, the generation of mouse models is expensive and time consuming.

The hiPSC technology allows the generation of patient-specific pluripotent stem cells. The remarkable potential of self-renewal and differentiation capacities of hiPSCs allows us to 
propagate them in vitro almost indefinitely and to differentiate them into cell types of interest. In this way, personalized CMs could be potentially limitlessly generated, which would obviate the need for heterologous expression systems and circumvent species-specific variations.

\subsection{Application of human iPSCs in cardiac research}

The discovery of inducing pluripotency in somatic cells has opened a very exciting and promising field with regard to potential applications in medical research. Although the hiPSC technology still needs improvements and refinements, its contributions to disease modelling, drug screening and discovery, toxicity tests as well as cell transplantation studies are already well-recognized (Bellin et al. 2012; Fig. 5).

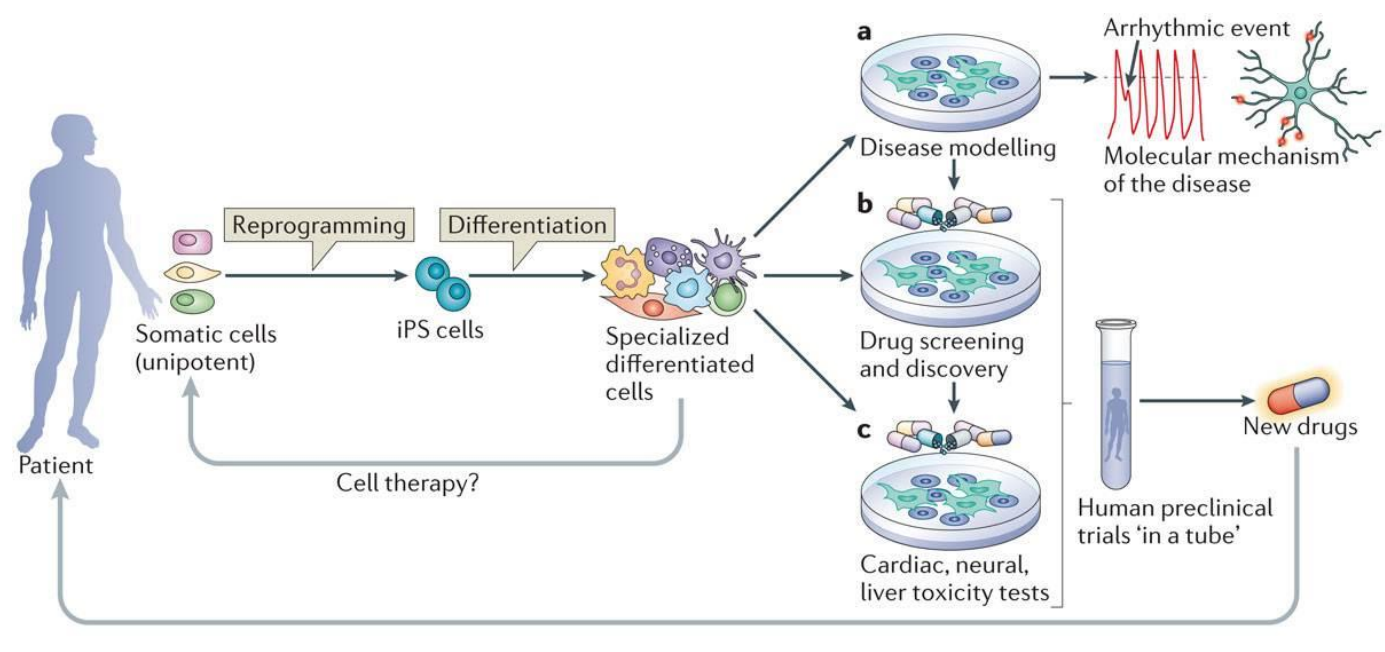

Nature Reviews | Molecular Cell Biology

Figure 5. Promises of hiPSCs in medical research. Generated hiPSCs from a patient with a degenerative disease could be used for cellular therapy by autologous transplantation to repair degenerated or damaged tissues. Another medical use is to derive hiPSCs from patients with genetically inherited or other disorders, differentiate them in vitro and get novel insights into the molecular mechanisms of the disease. Differentiated cells of interest can also provide platforms for toxicology testing and personalized drug development. (Figure taken from Bellin et al. 2012)

\subsubsection{Cell replacement therapy}

The hiPSC technology offers the possibility to treat many degenerative diseases, including diabetes, Alzheimer's disease, Parkinson's disease or cardiovascular diseases by autologous cell transplantation (Fig. 5). The risk of immune rejection after autologous transplantation would be minimized and the use of immunosuppressive drugs might become unnecessary. In contrast to human ESCs (hESCs), patient-specific hiPSCs circumvent ethical concerns regarding their origin. 
First therapeutic application studies with a mouse model of sickle cell anemia revealed promising results and provided a proof-of-concept illustration of the therapeutic use of iPSCs (Hanna et al. 2007). In this study, mice suffering from this disease could be rescued by autologous transplantation of iPSC-derived hematopoietic progenitor cells after the correction of the mutated hemoglobin allele by homologous recombination.

Studies like this may support the idea of using hiPSCs for heart regeneration. Recent work showed that hiPSC-derived CMs from a heart failure patient were able to engraft, survive, and integrate structurally with the host CMs after transplantation into rat hearts (Zwi-Dantsis et al. 2013). However, the correction of gene defects in (h)iPSCs remains challenging. More research focus has to be applied to improve efficiencies of gene correction technologies such as the use of zinc-finger nucleases (Wang et al. 2012), transcription activator-like effector nucleases (Hockemeyer et al. 2011), or clustered regularly interspaced short palindromic repeats (Mali et al. 2013) that induce DNA double-stranded breaks, followed by subsequent homology directed repair. Further investigation is necessary to ensure that the use of hiPSCs in cellular therapy is safe for patients and applicable in future.

\subsubsection{Disease modelling}

Patient-specific hiPSCs as a renewable and unlimited source for CMs also provide the possibility to study the pathophysiology of specific genetically inherited cardiac diseases in vitro (Fig. 5). Here, patient-specific hiPSC-derived CMs can act as a complementary model system to get a deeper insight into the molecular and electrophysiological mechanisms of arrhythmic syndromes. Patient-specific hiPSCs have already been generated from a wide spectrum of cardiac channelopathies, including LQTS type 1 (Moretti et al. 2010), type 2 (Itzhaki et al. 2011; Matsa et al. 2011; Lahti et al. 2012), and type 3 (Ma et al. 2013; Terrenoire et al. 2013), Timothy syndrome (Yazawa et al. 2011), and catecholaminergic polymorphic ventricular tachycardia (CPVT, Fatima et al. 2011; Novak et al. 2012). All of these hiPSC models showed that the patient-specific hiPSC-derived CMs could recapitulate the disturbed electrophysiological phenotype of the arrhythmia syndromes in vitro.

Currently, four different (h)iPSC models have been generated for studying SCN5A mutationrelated sodium channelopathies. The first iPSC model was generated from mouse embryonic fibroblasts (MEFs) of a $S c n 5 a^{\mathrm{\Lambda /+}}$ mouse model ( $\triangle \mathrm{KPQ}$ ), showing that $S c n 5 a^{\mathrm{\Delta} /+}$ iPSC-derived CMs could recapitulate the typical pathophysiological phenotype of LQTS type 3 in vitro 
(Malan et al. 2011). In another study, Davis and colleagues generated iPSCs from tail tip fibroblasts of the $S c n 5 a^{1798 \mathrm{ins} /+}$ mouse, and differentiated them into CMs. They showed that Scn $5 a^{1798 \mathrm{ins} /+}$ iPSC-derived CMs exhibited features of both loss-of-function (reduced $I_{N a}$ density) and gain-of-function (larger persistent $I_{N a}$ ), mirroring the defects observed in primary adult CMs isolated from the $S c n 5 a^{1798 \mathrm{ins} /+}$ mouse (Davis et al. 2012). Subsequently, hiPSCs were generated from a patient carrying the equivalent $S C N 5 A^{1795 i n s /++}$ mutation in this study, and patch clamp measurements on derivative CMs revealed the biophysical abnormalities similar to those in mouse Scn5a $a^{1798 \mathrm{ins} /+}$ iPSC-derived CMs. It is interesting to note that the $S C N 5 A^{1795 i n s \mathrm{D} /+}$ mutation clinically gives rise to an overlap phenotype of LQTS type 3 and $\mathrm{BrS}$ with conduction defects due to both gain- and loss-of-function effects on $\mathrm{Na}_{\mathrm{v}} 1.5$ (Bezzina et al. 1999). Moreover, hiPSCs carrying two other SCN5A mutations (p.F1473C, p.V1763M) were derived from patients with LQTS type 3 (Ma et al. 2013; Terrenoire et al. 2013). CMs derived from these hiPSCs showed significantly prolonged APD and enhanced persistent $I_{N a}$, recapitulating the typical pathophysiological phenotype of LQTS type 3. All of these studies indicate that (h)iPSC-derived CMs are suitable for studying complex sodium channel mutations in vitro. To our knowledge, no hiPSCs models have been reported regarding $\mathrm{BrS}$ associated with a $S C N 5 A$ mutation.

\subsubsection{Drug discovery and toxicity tests}

In the last decade, novel drug discovery, development, and safety testing consisted of an arduous and expensive process. In 2001, drug development was abandoned because of lack of efficacy in $30 \%$ of the medicines that entered clinical trials, and in another $30 \%$ because of safety concerns such as cardiotoxicity and hepatotoxicity (Laustriat et al. 2010).

One major reason for the difficult translation of drug discovery from molecular levels and animal models to human therapeutics is the lack of economical and reliable methods that can accurately mimic the human physiological response. So far, the success of preclinical phases of drug development is mainly based on animal models (Gunaseeli et al. 2010). For instance, a number of drugs have been developed that showed therapeutic effects in rodent models of amyotrophic lateral sclerosis. Unfortunately, all of them turned out to be ineffective in human patients, emphasizing the necessity of disease models using human cells (Groeneveld et al. 2003; Shefner et al. 2004). 
For the development of anti-arrhythmic drugs, hiPSC-derived CMs may be useful in filling the gap between animal models and clinical trials. Importantly, they exhibit many of the characteristics of normal in vivo CMs, including molecular, structural, and functional properties such as ion channel, transporter, and receptor expression, as well as similar electrophysiological properties and biochemical responses (Ma et al. 2011). Recent studies show that hiPSC-derived CMs respond to specific drugs in a similar way that the human heart responds (Dick et al. 2010). Due to the properties of disease-specific hiPSC-derived CMs (e. g. cells from patients with sodium channelopathies), their application would provide a unique and predictive model for the pre-clinical screening of candidate anti-arrhythmic pharmacological agents. In addition, the effective development of new drugs requires predictive toxicity assays of adequate accuracy during preclinical testing. Currently, CMs from animals are used in pre-clinical models for cardiac toxicity tests. However, pharmaceuticals are designed to act on human targets. Because of species-related differences, the increased risk of cardiotoxicity may not be recognized prior to clinical trials. Furthermore, the use of animals is costly and involves ethical concerns. Differentiated CMs derived from hiPSCs may provide an alternative source for cardiac toxicity tests.

\subsection{Aim of this thesis}

The aim of this thesis was to establish an in vitro cell culture system using the hiPSCtechnology as a disease model to study the pathophysiological and molecular mechanisms of BrS putatively caused by the SCN5A point mutation C5435A (p.S1812X). The scientific and technological objectives of the work included:

(1) Generation and characterization of hiPSCs from the BrS patient and control hiPSCs from a donor without a known inherited cardiac disease.

(2) Differentiation of the BrS- and Ctrl-hiPSCs into functional CMs.

(3) Electrophysiological phenotype characterization of the BrS- compared to CtrlhiPSC-derived CMs (BrS-CMs, Ctrl-CMs).

(4) Effects of experimental drugs on the function of hiPSC-derived CMs.

(5) Analysis of $S C N 5 A$ expression on mRNA and protein level in BrS- compared to Ctrl-CMs. 


\section{Materials and methods}

\subsection{Materials}

\subsubsection{Cells}

Mouse embryonic fibroblasts (MEFs): isolated from 15- to 17-day-old embryos of NMRI mice (Central Animal Facility, Universiy Medical Center Göttingen)

Human mesenchymal stem cells (hMSCs): BM69 >> isolated from bone marrow aspirate of a 18-year-old male patient (without known cardiac disease) left from diagnostic purposes, Department of Accident Surgery, Universiy Medical Center Göttingen; BM76 >> isolated from bone marrow aspirate of a 45-year-old female patient (without known cardiac disease) left from diagnostic purposes, Department of Hematology and Oncology, Universiy Medical Center Göttingen; BM77 >> obtained from bone marrow aspirate of a 50-year-old male BrS patient, Department of Cardiology and Pneumology, Universiy Medical Center Göttingen

Human embryonic stem cells (hESCs): stem cell line HES03 generated by ES Cell International (Singapore) and imported from NIH National Stem Cell Bank

Human induced pluripotent stem cells (hiPSCs): generated from hMSCs (BM69, BM76, BM77) with single lentivirus particles (OCT4, SOX2, LIN28, NANOG) or STEMCCA lentivirus; proved by the Institutional Ethical Committee (21/2/10), Universiy Medical Center Göttingen

\subsubsection{Oligonucleotides}

All oligonucleotides used for polymerase chain reaction (PCR) were purchased from Eurofins MWG Operon. The sequences are listed in alphabetical order (Table 1) together with the amplified fragment length $(\mathrm{F})$, annealing temperature $\left(\mathrm{T}_{\mathrm{A}}\right)$, and number of cycles $(\mathrm{C})$. 
Table 1. Oligonucleotides used for DNA sequencing and PCR analyses

\begin{tabular}{|c|c|c|c|c|}
\hline Gene & Sequence & F [bp] & $\mathbf{T}_{\mathbf{A}}\left[{ }^{\circ} \mathbf{C}\right]$ & C \\
\hline$A F P$ & $\begin{array}{l}\text { for: 5'-ACT CCA GTA AAC CCT GGT GTT G-3' } \\
\text { rev: 5'-GAA ATC TGC AAT GAC AGC CTC A-3' }\end{array}$ & 255 & 60 & 33 \\
\hline$A L B$ & $\begin{array}{l}\text { for: 5'-CCT TTG GCA CAA TGA AGT GGG TAA CC-3' } \\
\text { rev: 5'-CAG CAG TCA GCC ATT TCA CCA TAG G-3' }\end{array}$ & 284 & 62 & 35 \\
\hline$\alpha-M H C$ & $\begin{array}{l}\text { for: 5'-GTC ATT GCT GAA ACC GAG AAT G-3' } \\
\text { rev: 5'-GCA AAG TAC TGG ATG ACA CGC T-3' }\end{array}$ & 413 & 60 & 35 \\
\hline CASQ2 & $\begin{array}{l}\text { for: 5'-GGT CAC GCA AAA ACA GTT CC-3' } \\
\text { rev: 5'-CGA AGG CTT GGA CTT CCA GT-3' }\end{array}$ & 284 & 60 & 40 \\
\hline$c T N T$ & $\begin{array}{l}\text { for: 5’-GAC AGA GCG GAA AAG TGG GA-3' } \\
\text { rev: 5'-TGA AGG AGG CCA GGC TCT AT-3' }\end{array}$ & 305 & 55 & 35 \\
\hline$F O X D 3$ & $\begin{array}{l}\text { for: 5’-GTG AAG CCG CCT TAC TCG TAC-3’’ } \\
\text { rev: 5'-CCG AAG CTC TGC ATC ATG AG-3' }\end{array}$ & 353 & 61 & 38 \\
\hline GAPDH & $\begin{array}{l}\text { for: 5'-AGA GGC AGG GAT GAT GTT CT-3' } \\
\text { rev: 5'-TCT GCT GAT GCC CCC ATG TT-3'” }\end{array}$ & 265 & 55 & 34 \\
\hline GDF3 & $\begin{array}{l}\text { for: 5'-TTC GCT TTC TCC CAG ACC AAG GTT TC-3' } \\
\text { rev: 5'-TAC ATC CAG CAG GTT GAA GTG AAC AGC ACC-3' }\end{array}$ & 331 & 54 & 32 \\
\hline LIN28 & $\begin{array}{l}\text { for: 5'-AGT AAG CTG CAC ATG GAA GG-3' } \\
\text { rev: 5'-ATT GTG GCT CAA TTC TGT GC-3' }\end{array}$ & 410 & 52 & 36 \\
\hline NANOG & $\begin{array}{l}\text { for: 5'-AGT CCC AAA GGC AAA CAA CCC ACT TC-3' } \\
\text { rev: 5'-ATC TGC TGG AGG CTG AGG TAT TTC TGT CTC-3' }\end{array}$ & 164 & 64 & 36 \\
\hline $\begin{array}{l}S C N 5 A \\
(1)\end{array}$ & $\begin{array}{l}\text { for: 5'-TCA ACT TCC AGA CCT TCG CC-3' } \\
\text { rev: 5'-CGA TAC GGA GTG GCT CAG AC-3' }\end{array}$ & 408 & 60 & 35 \\
\hline $\begin{array}{l}S C N 5 A \\
(2)\end{array}$ & $\begin{array}{l}\text { for: 5'- GAG AGC ACC GAG CCC CTG AGT GAG G-3' } \\
\text { rev: 5'-CAC CAT GGG CAG GTC CAT GTT GAT G-3' }\end{array}$ & 189 & 59 & 35 \\
\hline SYP & $\begin{array}{l}\text { for: 5'-GCC TGT CTC CTT GAA CAC GAA C-3' } \\
\text { rev: 5'-TAC CGA GAG AAC AAC AAA GGG C-3' }\end{array}$ & 288 & 56 & 35 \\
\hline$T H$ & $\begin{array}{l}\text { for: 5'-GCG GTT CAT TGG GCG CAG G-3' } \\
\text { rev: 5'-CAA ACA CCT TCA CAG CTC G-3' }\end{array}$ & 215 & 60 & 34 \\
\hline
\end{tabular}

$\boldsymbol{A F P}$ : alpha-1-fetoprotein, $\boldsymbol{A L B}$ : albumin, $\boldsymbol{\alpha}-\boldsymbol{M H C}$ : myosin heavy chain (alpha), $\boldsymbol{C A S Q 2 :}$ calsequestrin 2, $\boldsymbol{c T N T}$ : cardiac troponin T, $\boldsymbol{F O X D 3}$ : forkhead box D3, GAPDH: glyceraldehyde-3-phosphate dehydrogenase, GDF3: growth differentiation factor 3, SYP: synaptophysin, $\boldsymbol{T H}$ : tyrosine hydroxylase

\subsubsection{STEMCCA lentivirus}

Human iPSCs were generated from hMSCs using the humanized single polycistronic lentiviral "stem cell cassette" (STEMCCA) system (kindly provided by Prof. Kotton, Boston University School of Medicine). This vector contains all four Yamanaka factors OCT4, SOX2, $K L F 4$, and $c-M Y C$, separated by the self-cleaving 2A peptide and internal ribosome entry site (IRES) sequences, driven by a constitutive elongation factor-1 alpha (EF-1 $\alpha)$ promoter (Fig. 6). The STEMCCA lentivirus contains also loxP sites for a potential Cre-mediated removal of the transgenes after successful reprogramming. However, even after excision, around $200 \mathrm{bp}$ of an inactive viral long terminal repeat site remains in the host genome, hence the risk of insertional mutagenesis may not be completely eliminated (Somers et al. 2010). 


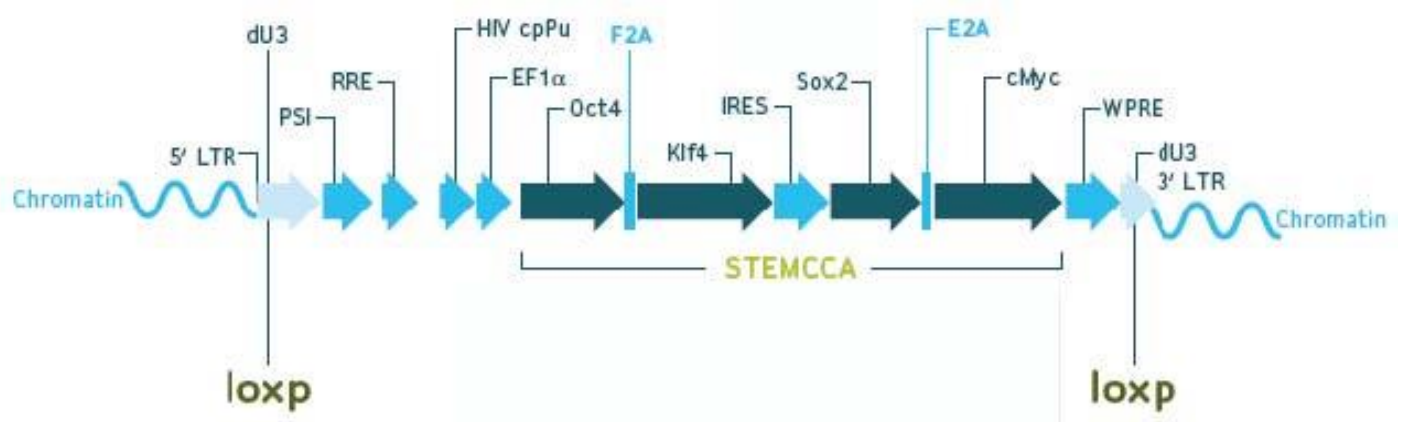

Figure 6. Excisable human STEMCCA lentivirus containing the reprogramming factors $O C T 4, K L F 4$, $S O X 2$, and $\boldsymbol{c}-\boldsymbol{M Y C}$. The factors are separated by $2 \mathrm{~A}$ peptide and IRES sequences and driven by the $E F-1 \alpha$ constitutive promoter. The vector is flanked with loxP sites. (Figure taken from Merck Millipore)

\subsubsection{Antibodies}

Table 2. Primary antibodies used for immunofluorescence (IF) and Western blot (WB) analyses

\begin{tabular}{|c|c|c|c|c|}
\hline \multirow[t]{2}{*}{ Antigen } & \multirow[t]{2}{*}{ Type } & \multirow[t]{2}{*}{ Supplier } & \multicolumn{2}{|c|}{ Dilution } \\
\hline & & & IF & WB \\
\hline AFP & polyclonal rabbit IgG & Dako, A0008 & $1: 100$ & - \\
\hline$\alpha$-actinin & mouse IgG1 & Sigma-Aldrich $^{\circledR}$, A7811 & $1: 1000$ & $1: 10000$ \\
\hline class III $\beta$-tubulin & mouse $\operatorname{IgG} 2 \mathrm{~A}$ & Covance $^{\circledR}$, MMS-435P & $1: 2000$ & - \\
\hline cTNT & mouse IgG1 & Thermo Scientific, MS295PABX & $1: 200$ & - \\
\hline CamKII & rabbit $\operatorname{IgG}$ & D. Bers, University of California & - & $1: 12000$ \\
\hline Cx43 & rabbit $\operatorname{IgG}$ & Abcam $^{\circledR}$, ab11370 & $1: 1000$ & - \\
\hline GAPDH & mouse $\operatorname{IgG}$ & Biotrend, BT46-9995-55 & - & $1: 50000$ \\
\hline LIN28 & goat $\operatorname{IgG}$ & R\&D Systems ${ }^{\circledR}$, AF3757 & $1: 300$ & - \\
\hline MLC2a & mouse $\operatorname{IgG} 2 \mathrm{~B}$ & Synaptic Systems, 311-011 & $1: 200$ & - \\
\hline NANOG & goat $\operatorname{IgG}$ & R\&D Systems ${ }^{\circledR}$, AF1997 & $1: 200$ & - \\
\hline $\mathrm{Na}_{\mathbf{v}} 1.5(1)$ & mouse IgM & Abcam $^{\circledR}$, ab62388 & $1: 100$ & failed \\
\hline $\mathrm{Na}_{\mathbf{v}} 1.5(2)$ & polyclonal rabbit IgG & Alomone Labs, ASC-005 & - & failed \\
\hline $\mathrm{Na}_{\mathbf{v}} 1.5(3)$ & polyclonal guinea pig & Alomone Labs, AGP-008 & - & failed \\
\hline $\mathrm{Na}_{\mathbf{v}} 1.5(4)$ & polyclonal rabbit IgG & Alomone Labs, ASC-013 & - & $1: 2000$ \\
\hline OCT4 & goat IgG & R\&D Systems ${ }^{\circledR}$, AF1759 & $1: 40$ & - \\
\hline SMA & mouse $\operatorname{IgG} 2 \mathrm{~A}$ & Sigma-Aldrich $^{\circledR}$, A2547 & $1: 3000$ & - \\
\hline SOX2 & mouse $\operatorname{IgG} 2 \mathrm{~A}$ & R\&D Systems ${ }^{\circledR}$, MAB2018 & $1: 50$ & - \\
\hline SSEA4 & mouse $\operatorname{IgG}$ & Abcam $^{\circledR}$, ab16287 & $1: 100$ & - \\
\hline TRA-1-60 & mouse IgM & Abcam $^{\circledR}$, ab16288 & $1: 200$ & - \\
\hline
\end{tabular}

CamKII: $\mathrm{Ca}^{2+} /$ calmodulin-dependent protein kinase II, Cx43: connexin 43, MLC2a: myosin light chain 2 (atrial), SMA: smooth muscle actin, SSEA4: stage-specific embryonic antigen 4 
Table 3. Secondary antibodies

\begin{tabular}{llc}
\hline \hline Antibody & Supplier & Dilution \\
\hline \hline Alexa 488-conjugated goat- $\alpha$-mouse IgG & Life Technologies ${ }^{\text {TM }, ~ A-11001 ~}$ & $1: 200$ \\
Cy3-conjugated donkey- $\alpha$-goat IgG & Jackson ImmunoResearch, 705-166-147 & $1: 600$ \\
Cy3-conjugated goat- $\alpha$-mouse IgG + IgM & Jackson ImmunoResearch, 115-165-068 & $1: 300$ \\
Cy3-conjugated goat- $\alpha$-rabbit IgG & Jackson ImmunoResearch, 111-165-003 & $1: 800$ \\
FITC-conjugated donkey- $\alpha$-rabbit IgG & Jackson ImmunoResearch, 711-095-152 & $1: 200$ \\
FITC-conjugated goat- $\alpha$-mouse IgG & Jackson ImmunoResearch, 115-096-072 & $1: 200$ \\
FITC-conjugated goat- $\alpha$-mouse IgM & Jackson ImmunoResearch, 115-095-020 & $1: 100$ \\
HRP-conjugated donkey- $\alpha$-rabbit IgG & GE Healthcare, NA934 & $1: 10000$ \\
HRP-conjugated goat- $\alpha$-mouse IgG + IgM & Jackson ImmunoResearch, 115-036-068 & $1: 10000$ \\
HRP-conjugated sheep- $\alpha$-mouse IgG & GE Healthcare, NA931V & $1: 20000$ \\
\hline \hline
\end{tabular}

\subsubsection{Media, solutions, and chemicals for cell culture}

Table 4. Components for cell culture

\begin{tabular}{|c|c|}
\hline Components & Supplier \\
\hline Accutase $^{\mathrm{TM}}$ & PAA Laboratories \#L11-007 \\
\hline B-2 $7^{\circledR}$ serum free supplement $(50 x)$ & Life Technologies ${ }^{\mathrm{TM}} \# 17504044$ \\
\hline B- $27^{\circledR}$ serum free supplement (w/o insulin, 50x) & Life Technologies ${ }^{\mathrm{TM}} \# 0050129 \mathrm{SA}$ \\
\hline$\beta$-mercaptoethanol & Serva Electrophoresis \#28625 \\
\hline Bovine albumin fraction $\mathrm{V}$ solution (BSA, $7.5 \%$ ) & Life Technologies ${ }^{\mathrm{TM}} \# 15260037$ \\
\hline CHIR99021 & Merck Millipore \#361559 \\
\hline Collagenase type 2 & Worthington ${ }^{\circledR}$ \#LS004176 \\
\hline Collagenase type 4 & Worthington ${ }^{\circledR}$ \#LS004189 \\
\hline Diltiazem hydrochloride & Sigma-Aldrich $^{\circledR}$ \#D2521 \\
\hline Dimethyl sulfoxide (DMSO) & Sigma-Aldrich $^{\circledR}$ \#D2650 \\
\hline Dulbeccós modified Eagle medium (DMEM) & Life Technologies ${ }^{\mathrm{TM}} \# 11960044$ \\
\hline Dulbecco’s modified Eagle medium/F-12 & Life Technologies $^{\mathrm{TM}} \# 31331028$ \\
\hline Dulbecco's phosphate buffered saline (DPBS) & Life Technologies ${ }^{\mathrm{TM}} \# 14190094$ \\
\hline Ethylenediaminetetraacetic acid (EDTA) & Sigma-Aldrich $^{\circledR} \#$ E6758 \\
\hline Essential $8^{\mathrm{TM}}$ basal medium & Life Technologies ${ }^{\mathrm{TM}}$ \#A1516901 \\
\hline Essential $8^{\mathrm{TM}}$ supplement & Life Technologies ${ }^{\mathrm{TM}}$ \#A1517101 \\
\hline Fetal bovine serum (FBS) & $\begin{array}{l}\text { Sigma-Aldrich }^{\circledR} \# \text { F7524 / } \\
\text { Lonza \#DE14802F }\end{array}$ \\
\hline Fibroblast growth factor basic (bFGF) & PeproTech \#100-18B \\
\hline Gelatin & Sigma-Aldrich $^{\circledR} \# 48720$ \\
\hline
\end{tabular}




\begin{tabular}{|c|c|}
\hline Geltrex $^{\mathrm{TM}}$ & Life Technologies ${ }^{\mathrm{TM}}$ \#A1413301 \\
\hline HEPES sodium salt solution (1 M) & Sigma-Aldrich ${ }^{\circledR} \# \mathrm{H} 3662$ \\
\hline Iscove's modified Dulbecco's medium (IMDM) & Life Technologies ${ }^{\mathrm{TM}} \# 31980022$ \\
\hline Isopropanol & Merck Millipore \#1096341000 \\
\hline IWP2 & Merck Millipore \#681671 \\
\hline Knockout $^{\mathrm{TM}}$ serum replacement & Life Technologies ${ }^{\mathrm{TM}} \# 10828028$ \\
\hline L-glutamine (200 mM) & Life Technologies ${ }^{\mathrm{TM}} \# 25030024$ \\
\hline Lymphocyte separation medium 1077 & PAA \#J11-004 \\
\hline Mitomycin C & Serva Electrophoresis \#29805.02 \\
\hline Monothioglycerol (MTG) & Sigma-Aldrich ${ }^{\circledR} \#$ M6145-25ML \\
\hline Non-essential amino acids (NEAA, 100x) & Life Technologies ${ }^{\mathrm{TM}} \# 11140035$ \\
\hline Penicillin-streptomycin solution (100x) & Life Technologies ${ }^{\mathrm{TM}} \# 15140-122$ \\
\hline Polybrene (hexadimethrine bromide) & Sigma-Aldrich $^{\circledR} \# 107689$ \\
\hline Pro-survival compound & Merck Millipore \#529659 \\
\hline Roswell Park Memorial Institute (RPMI 1640) medium & Life Technologies ${ }^{\mathrm{TM}} \# 72400-021$ \\
\hline RPMI 1640 (w/o glucose) & Life Technologies ${ }^{\mathrm{TM}} \# 11879-020$ \\
\hline Sodium DL-lactate solution $60 \%$ (w/w) & Sigma-Aldrich $^{\circledR} \#$ L4263 \\
\hline (S)-(-)-Bay K8644 & Sigma-Aldrich $^{\circledR} \#$ B133 \\
\hline Trypsin & Life Technologies ${ }^{\mathrm{TM}} \# 27250-018$ \\
\hline
\end{tabular}

bFGF: dissolved in $0.1 \% \mathrm{v} / \mathrm{v}$ BSA/DPBS to a stock solution of $10 \mathrm{ng} / \mu \mathrm{l}$

$\beta$-mercaptoethanol (100x for cell culture): $7 \mu \mathrm{l}$ diluted in $10 \mathrm{ml}$ DPBS and sterile filtrated with Steriflip ${ }^{\circledR}$ filters $(0.22 \mu \mathrm{m}$, Merck Millipore \#SCGP00525)

BSA/DPBS (1 \% w/v): $1 \mathrm{ml}$ of $7.5 \% \mathrm{w} / \mathrm{v}$ BSA added to $6.5 \mathrm{ml}$ DPBS

Cardiac selection medium (100 ml): $100 \mathrm{ml}$ RPMI 1640 (w/o glucose), $0.4 \mathrm{ml}$ of $1 \mathrm{M}$ lactate/ HEPES solution

CHIR99021 (12 mM stock solution): $5 \mathrm{mg}$ dissolved in $0.894 \mathrm{ml}$ DMSO, stored at $-20{ }^{\circ} \mathrm{C}$

Collagenase type 2: dissolved in DMEM/F12 to a working solution of $300 \mathrm{U} / \mathrm{ml}$, sterile filtrated, and stored at $-20{ }^{\circ} \mathrm{C}$

Collagenase type 4: dissolved in DMEM/F12 to a working solution of $200 \mathrm{U} / \mathrm{ml}$, sterile filtrated, and stored at $-20{ }^{\circ} \mathrm{C}$ 
Differentiation medium I (100 ml): 98 ml RPMI 1640, 2 ml B-27 $7^{\circledR}$ serum free supplement (w/o insulin)

Differentiation medium II (100 ml): 98 ml RPMI 1640, 2 ml B-27 $7^{\circledR}$ serum free supplement

EDTA (50x): $1 \mathrm{~g}$ EDTA dissolved in $100 \mathrm{ml}$ DPBS; pH adjusted to 7.0 with $1 \mathrm{M} \mathrm{NaOH}$; sterile filtrated and stored at $4{ }^{\circ} \mathrm{C}$

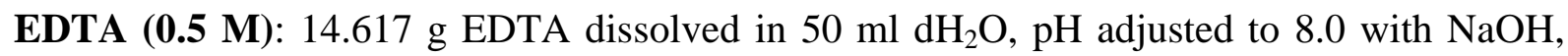
filled up to $100 \mathrm{ml}$ with $\mathrm{dH}_{2} \mathrm{O}$, sterile filtrated and stored at $-20{ }^{\circ} \mathrm{C}$

EDTA dissociation solution: $500 \mu \mathrm{l}$ EDTA $(0.5 \mathrm{M})$ in $500 \mathrm{ml}$ DPBS, $0.9 \mathrm{~g} \mathrm{NaCl}$ to adjust the osmolarity to $340 \mathrm{mOsm}$; sterile filtrated and stored at $4{ }^{\circ} \mathrm{C}$

Essential $8^{\mathrm{TM}}$ medium $(\mathbf{1 0 0} \mathbf{~ m l}): 99 \mathrm{ml}$ essential $8^{\mathrm{TM}}$ basal medium, $1 \mathrm{ml}$ essential $8^{\mathrm{TM}}$ supplement

FBS: heat inactivated for $30 \mathrm{~min}$ at $56{ }^{\circ} \mathrm{C}$

Feeder layer medium (100 ml): 84 ml DMEM, 15 ml FBS, 1 ml L-glutamine

Freezing medium (100 $\mathbf{~ m l ) : ~} 72 \mathrm{ml}$ DMEM, $20 \mathrm{ml}$ FBS, $8 \mathrm{ml}$ DMSO

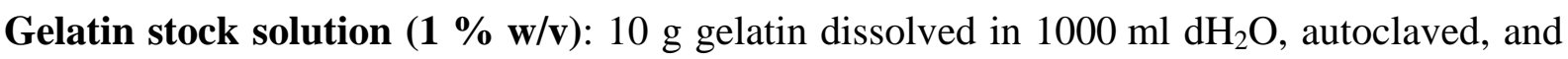
stored at $4{ }^{\circ} \mathrm{C}$

Geltrex $^{\text {TM: }} 2 \mathrm{mg}$ aliquoted and stored at $-80{ }^{\circ} \mathrm{C}$; dissolved in $12 \mathrm{ml}$ cold DMEM/F12 before use

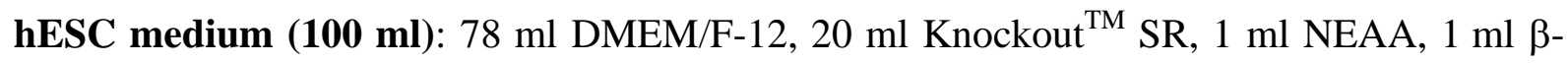
mercaptoethanol (100x), $10 \mathrm{ng} / \mathrm{ml}$ medium bFGF

hMSC medium (100 ml): 77 ml DMEM, 20 ml FBS, 1 ml NEAA, 1 ml L-glutamine, 1 ml $\beta$ mercaptoethanol (100x), $10 \mathrm{ng} / \mathrm{ml}$ medium bFGF

Iscove's medium (100 ml): 79 ml IMDM, 20 ml FBS, 1 ml NEAA, $450 \mu$ M MTG (freshly prepared)

IWP2 (5 mM stock solution): $10 \mathrm{mg}$ dissolved in $4.28 \mathrm{ml}$ DMSO and incubated at $37{ }^{\circ} \mathrm{C}$ for $10 \mathrm{~min}$, stored at $-20^{\circ} \mathrm{C}$ 
Lactate/HEPES (1 M stock solution): $3 \mathrm{ml}$ of $60 \% \mathrm{w} / \mathrm{w}$ sodium DL-lactate solution diluted in $18 \mathrm{ml}$ of $1 \mathrm{M}$ HEPES sodium salt solution, store at $-20^{\circ} \mathrm{C}$

Mitomycin C stock solution: dissolved in DPBS $(200 \mu \mathrm{g} / \mathrm{ml})$ and stored at $-20{ }^{\circ} \mathrm{C}$

MTG (150 mM): $13 \mu \mathrm{l} \mathrm{MTG} \mathrm{diluted} \mathrm{in} 1 \mathrm{ml}$ IMDM and sterile filtrated with Millex ${ }^{\circledR}$-GS filter $(0.22 \mu \mathrm{m}$, Merck Millipore \#SLGS033SS), freshly prepared before use

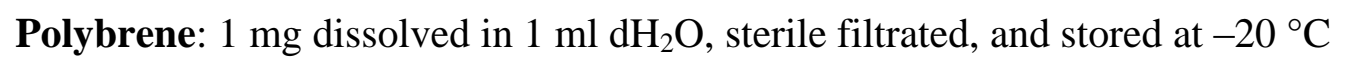

Pro-survival compound (5 mM stock solution): $10 \mathrm{mg}$ dissolved in $6.8 \mathrm{ml} \mathrm{DMSO}$, stored at $-20{ }^{\circ} \mathrm{C}$

Trypsin (0.2 \% w/v): $2 \mathrm{~g}$ trypsin dissolved in $1000 \mathrm{ml}$ DPBS; sterile filtrated with Steritop ${ }^{\mathrm{TM}_{-} \mathrm{GP}}$ filters $(0.22 \mu \mathrm{m}$, Merck Millipore \#SCGPT02RE)

Trypsin/EDTA $(0.1 \%$ w/v): $0.2 \%$ w/v trypsin mixed in a ratio $1: 1$ with $1 x$ EDTA (50x EDTA diluted 1/50 with DPBS); stored at $4{ }^{\circ} \mathrm{C}$

\subsubsection{Solutions, buffers, and chemicals for molecular biological, and protein analyses}

Table 5. Components for molecular biological methods, and protein analyses

\begin{tabular}{|c|c|}
\hline Components & Supplier \\
\hline $\operatorname{Agar}^{\mathrm{TM}}$ & Serva Electrophoresis \#200201 \\
\hline Agencourt $^{\circledR}$ AMPure ${ }^{\circledR}$ XP PCR purification kit & Beckman Coulter \#A63882 \\
\hline Alkaline phosphatase staining kit & Sigma-Aldrich ${ }^{\circledR} \# 86 \mathrm{R}-1 \mathrm{KT}$ \\
\hline Ammonium persulfate (APS, $\left.\left(\mathrm{NH}_{4}\right)_{2} \mathrm{~S}_{2} \mathrm{O}_{8}\right)$ & $\operatorname{Roth}^{\circledR} \# 9178$ \\
\hline AmpliTaq ${ }^{\circledR}$ DNA polymerase with buffer II & Life Technologies ${ }^{\mathrm{TM}}$ \#N808-0167 \\
\hline Boric acid & Sigma-Aldrich $^{\circledR} \# 15663$ \\
\hline Bromphenol blue & Sigma-Aldrich ${ }^{\circledR}$ \#B0126 \\
\hline cOmplete (protease inhibitor cocktail tablets) & Roche \# 04693132001 \\
\hline DAPI (4', 6-diamidino-2-phenylindole dihydrochloride) & Sigma-Aldrich ${ }^{\circledR}$ \#D9542 \\
\hline DEPC-treated water & Ambion ${ }^{\circledR} \#$ AM9915G \\
\hline Dithiothreitol (DTT) & Roth $^{\circledR} \# 6908$ \\
\hline dNTP mix & Bioline \#BIO-39029 \\
\hline Ethidium bromide & $\operatorname{Roth}^{\circledR} \# 2218$ \\
\hline Fluoromount-GTM & eBioscience \#00-4958-02 \\
\hline Formalin $(37 \%)$ & Merck Millipore \#1039991000 \\
\hline
\end{tabular}




\begin{tabular}{|c|c|}
\hline GeneRead library quant kit & Qiagen \# 180612 \\
\hline GeneRuler $^{\mathrm{TM}} 100$ bp Plus DNA Ladder & Thermo Scientific \#0321 \\
\hline Giemsa stain & Sigma-Aldrich ${ }^{\circledR}$ \#GS500 \\
\hline Glacial acetic acid & Merck Millipore \#1.00063.1000 \\
\hline Glycerin $(87 \%)$ & Merck Millipore \#4094 \\
\hline Glycine & Serva Electrophoresis \#23391 \\
\hline GoTaq $^{\circledR}$ DNA polymerase & Promega \#M3175 \\
\hline Immobilon ${ }^{\mathrm{TM}}$ Western chemiluminescent HRP substrate & Merck Millipore \#WBKLS0500 \\
\hline Karyomax ${ }^{\circledR}$ Colcemid ${ }^{\circledR}$ solution (demecolcine) & Life Technologies ${ }^{\mathrm{TM}} \# 15210-040$ \\
\hline Maxwell $^{\circledR} 16$ cell DNA purification kit & Promega \#AS1020 \\
\hline Methanol & J. T. Baker \#8402 \\
\hline MuLV reverse transcriptase $(50 \mathrm{U} / \mu \mathrm{l})$ & Life Technologies ${ }^{\mathrm{TM}}$ \#N808-0018 \\
\hline Nonfat dry milk & TSI GmbH \& Co \\
\hline Oligo d(T) ${ }_{16}(50 \mu \mathrm{M})$ & Life Technologies ${ }^{\mathrm{TM}}$ \#N808-0128 \\
\hline Paraformaldehyde (PFA) & Sigma-Aldrich $^{\circledR} \# 158127$ \\
\hline PeqGold protein marker $\mathrm{V}$ & Peqlab \#27-2210 \\
\hline PhosSTOP (phosphatase inhibitor cocktail tablets) & Roche \#04906837001 \\
\hline Pierce $^{\mathrm{TM}} \mathrm{BCA}$ protein assay kit & Thermo Scientific \#23225 \\
\hline Ponceau S solution & Sigma-Aldrich $^{\circledR} \#$ P7170-1L \\
\hline Potassium chloride $(\mathrm{KCl})$ & $\operatorname{Roth}^{\circledR} \# 6781$ \\
\hline QIAamp ${ }^{\circledR}$ DNA mini kit & Qiagen \#51304 \\
\hline QIAquick $^{\circledR}$ gel extraction kit & Qiagen \#28706 \\
\hline RNase inhibitor $(20 \mathrm{U} / \mu \mathrm{l})$ & Life Technologies ${ }^{\mathrm{TM}}$ \#N808-0119 \\
\hline Rotiphorese $^{\circledR}$ gel 30 & Roth $^{\circledR} \# 3029$ \\
\hline Sodium cloride $(\mathrm{NaCl})$ & Roth $^{\circledR}$ \#P3957 \\
\hline Sodium dihydrogen phosphate $\left(\mathrm{NaH}_{2} \mathrm{PO}_{4} \cdot \mathrm{H} 2 \mathrm{O}\right)$ & Merck Millipore \#1.06345 \\
\hline Sodium dodecyl sulfate (SDS; $\mathrm{NaC}_{12} \mathrm{H}_{25} \mathrm{SO}_{4}$ ) & Roth $^{\circledR} \# 2326$ \\
\hline Sodium fluoride $(\mathrm{NaF})$ & $\operatorname{Roth}^{\circledR} \#$ P756 \\
\hline Sodium hydrogen phosphate $\left(\mathrm{Na}_{2} \mathrm{HPO}_{4}\right)$ & Merck Millipore \#567547 \\
\hline SV total RNA isolation system & Promega \#Z3105 \\
\hline $\mathrm{SYBR}^{\circledR}$ Green PCR master mix & Life Technologies ${ }^{\mathrm{TM}} \# 4309155$ \\
\hline Tetramethylethylenediamine (TEMED, $\mathrm{C}_{6} \mathrm{H}_{16} \mathrm{~N}_{2}$ ) & $\operatorname{Roth}^{\circledR} \# 2367$ \\
\hline Tris & $\operatorname{Roth}^{\circledR} \# 5429$ \\
\hline Triton $^{\mathrm{TM}} \mathrm{X}-100$ & Sigma-Aldrich $^{\circledR} \# \mathrm{X}-100$ \\
\hline TrypLETM Express & Life Technologies ${ }^{\mathrm{TM}} \# 12604-013$ \\
\hline Tween 20 & Bio-Rad \#170-6531 \\
\hline
\end{tabular}


Agar gel (1.5 \% w/v): 1.5 g DNA Agar ${ }^{\mathrm{TM}}$ dissolved in $100 \mathrm{ml} 1 \mathrm{x}$ TB buffer by boiling in a microwave, $6 \mu \mathrm{l}$ of $10 \mathrm{mg} / \mathrm{ml}$ ethidium bromide was added to $100 \mathrm{ml}$ agar gel for visualization of DNA under ultraviolet light

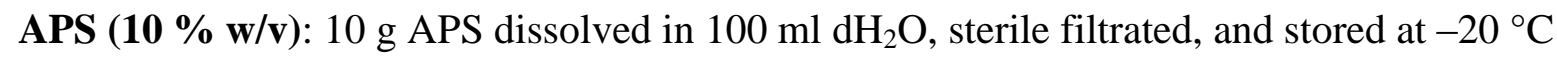

Blue loading buffer (5x): $31.25 \mathrm{ml} 1 \mathrm{M}$ Tris-HCl (pH 6.8), $10 \mathrm{~g}$ SDS, $5 \mathrm{mg}$ bromphenol blue, $57 \mathrm{ml}$ glycerin (87\%), filled up to $100 \mathrm{ml}$ with $\mathrm{dH}_{2} \mathrm{O} ; \mathrm{pH}$ adjusted to 6.8 , stored at $-20{ }^{\circ} \mathrm{C}$; before use, $40 \mu 1 \beta$-mercaptoethanol were added to $360 \mu \mathrm{l}$ buffer

Cell lysis buffer (10 ml): $100 \mu \mathrm{l} 2 \mathrm{M}$ Tris-HCl (pH 7.4), $500 \mu \mathrm{l} \mathrm{NaCl}(4 \mathrm{M}), 1 \mathrm{ml} \mathrm{NaF}$ (200 mM), $100 \mu 1$ Triton $^{\mathrm{TM}} \mathrm{X}-100,100 \mu \mathrm{Na}_{3} \mathrm{VO}_{4}(100 \mathrm{mM}), 100 \mu 1$ DTT (100 mM), $1 / 2$ tablet of cOmplete (EDTA-free), 1 tablet of PhosSTOP, filled up to $10 \mathrm{ml}$ with $\mathrm{dH}_{2} \mathrm{O}$

Fixation buffer: methanol (3) : glacial acetic acid (1) freshly prepared and cooled at $-20{ }^{\circ} \mathrm{C}$

Nonfat dry milk (5 \% w/v): 5 g nonfat dry milk, 100 ml 1x TBS-T buffer

PFA (4 \% w/v): 4 g PFA dissolved in $100 \mathrm{ml}$ DPBS under heated conditions, used for 1 week

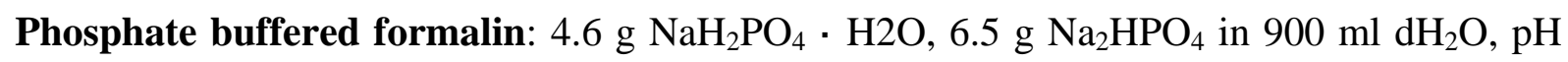
adjusted to 7.0 with $\mathrm{HCl} ; 89.2 \mathrm{ml}$ of buffer in $10.8 \mathrm{ml} 37 \%$ formalin; stored at $4{ }^{\circ} \mathrm{C}$

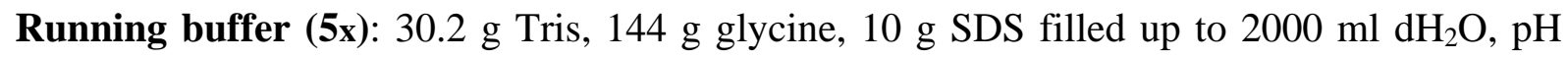
adjusted to 8.3 with $\mathrm{HCl}$

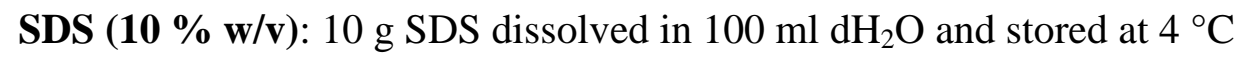

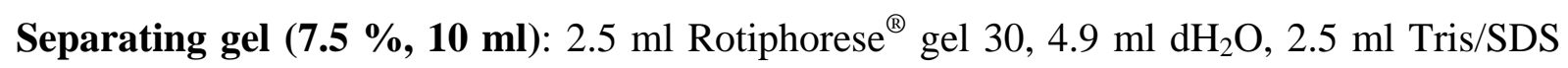
(4x, pH 8.8), $100 \mu 1$ APS (10\%), $10 \mu 1$ TEMED

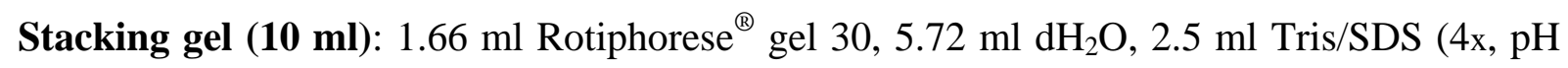
6.8), $100 \mu \mathrm{l}$ APS (10\%), $10 \mu 1$ TEMED

TB buffer (5x): $108 \mathrm{~g}$ Tris, $55 \mathrm{~g}$ boric acid, filled up to $2000 \mathrm{ml}$ with $\mathrm{dH}_{2} \mathrm{O}$

TBS buffer (10x): $48.4 \mathrm{~g}$ Tris, $58.48 \mathrm{~g} \mathrm{NaCl}$, filled up to $2000 \mathrm{ml}$ with $\mathrm{dH}_{2} \mathrm{O}, \mathrm{pH}$ 7.5; stored at $4{ }^{\circ} \mathrm{C}$

TBS-T buffer (1x): $500 \mathrm{ml}$ TBS buffer (10x), $5 \mathrm{ml}$ Tween 20, filled up to $5000 \mathrm{ml}$ with $\mathrm{dH}_{2} \mathrm{O}$ 
Transfer buffer (5x): $39.4 \mathrm{~g}$ Tris, $144 \mathrm{~g}$ glycine, filled up to $2000 \mathrm{ml}$ with $\mathrm{dH}_{2} \mathrm{O}$, pH adjusted to 8.3 with $\mathrm{HCl}$

Transfer buffer (1x, $20 \%$ v/v methanol): $400 \mathrm{ml}$ transfer buffer (5x), $400 \mathrm{ml}$ methanol, $2 \mathrm{ml} \mathrm{SDS} \mathrm{(10 \%} \mathrm{w/v)} \mathrm{filled} \mathrm{up} \mathrm{to} 2000 \mathrm{ml}$ with $\mathrm{dH}_{2} \mathrm{O}$

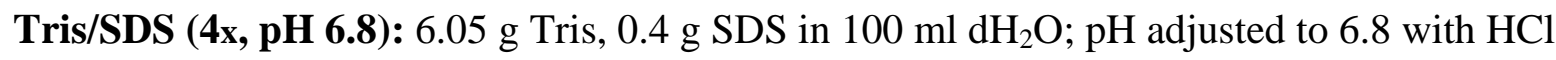

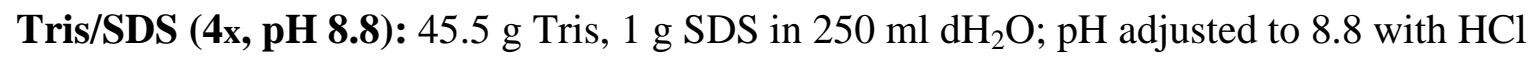

Triton $^{\text {TM }}$ X-100 $\left(0.1 \%\right.$ v/v): 1 ml Triton ${ }^{\mathrm{TM}}$ X-100 diluted in $999 \mathrm{ml}$ DPBS

\subsubsection{Solutions and chemicals for electrophysiological analyses}

Table 6. Chemicals for electrophysiological analyses

\begin{tabular}{|c|c|}
\hline Chemicals & Supplier \\
\hline Adenosine 5'-triphosphate magnesium salt (Mg-ATP) & Sigma-Aldrich ${ }^{\circledR}$ \#A9187 \\
\hline Calcium chloride $\left(\mathrm{CaCl}_{2}\right)$ & Sigma-Aldrich ${ }^{\circledR} \# 21115$ \\
\hline Cesium chloride $(\mathrm{CsCl})$ & Sigma-Aldrich ${ }^{\circledR}$ \#C3032 \\
\hline Cesium hydroxide solution $(\mathrm{CsOH})$ & Sigma-Aldrich $^{\circledR} \# 232041$ \\
\hline $\mathrm{D}(+)$-glucose & Merck Millipore \#108337 \\
\hline Ethylene glycol tetraacetic acid (EGTA) & Sigma-Aldrich $^{\circledR}$ \#E3889 \\
\hline Flecainide acetate salt & Sigma-Aldrich $^{\circledR} \# \mathrm{~F} 6777$ \\
\hline Guanosine 5'-triphosphate lithium salt (Li-GTP) & Sigma-Aldrich ${ }^{\circledR}$ \#G5884 \\
\hline HEPES & $\operatorname{Roth}^{\circledR} \# 9105$ \\
\hline L-aspartic acid potassium salt & Sigma-Aldrich ${ }^{\circledR} \#$ A6558 \\
\hline L-glumatic acid & Sigma-Aldrich ${ }^{\circledR} \# \mathrm{G} 1251$ \\
\hline Magnesium chloride $\left(\mathrm{MgCl}_{2}\right)$ & Sigma-Aldrich $^{\circledR} \# \mathrm{M} 8266$ \\
\hline Nifedipine & Sigma-Aldrich ${ }^{\circledR} \#$ N7634 \\
\hline Niflumic acid & Sigma-Aldrich $^{\circledR}$ \#N0630 \\
\hline Potassium chloride $(\mathrm{KCl})$ & $\operatorname{Roth}^{\circledR} \# 6781$ \\
\hline Potassium hydroxide (KOH) & $\operatorname{Roth}^{\circledR} \# 6751$ \\
\hline Quinidine & Sigma-Aldrich ${ }^{\circledR} \# \mathrm{Q} 3625$ \\
\hline Sodium chloride $(\mathrm{NaCl})$ & $\operatorname{Roth}^{\circledR} \# 3957$ \\
\hline Strophantidine & Sigma-Aldrich ${ }^{\circledR} \#$ S6626 \\
\hline Tetramethylammonium chloride (TMAC) & Sigma-Aldrich $^{\circledR} \# 87718$ \\
\hline $0.25 \%$ trypsine/EDTA solution & Life Technologies ${ }^{\mathrm{TM}} \# 25200-056$ \\
\hline
\end{tabular}


Pipette solution for AP measurements (in $\mathbf{m M}$ ): $122 \mathrm{~L}$-aspartic acid potassium salt, 10 $\mathrm{NaCl}, 8 \mathrm{KCl}, 1 \mathrm{MgCl}_{2}, 5 \mathrm{Mg}$-ATP, $0.3 \mathrm{Li}$-GTP, 10 HEPES; $\mathrm{pH}$ adjusted to 7.2 with $\mathrm{KOH}$

Pipette solution for $\boldsymbol{I}_{\mathrm{Na}}$ measurements (in $\mathbf{m M}$ ): $100 \mathrm{CsCl}, 40 \mathrm{Cs}$-glutamate (L-glumatic acid $+\mathrm{CsOH}$ ), $5 \mathrm{NaCl}, 0.92 \mathrm{MgCl}_{2}, 1$ EGTA, $5 \mathrm{Mg}$-ATP, $0.3 \mathrm{Li}-\mathrm{GTP}, 0.36 \mathrm{CaCl}_{2}$, 0.03 niflumic acid, 0.02 nifedipine, 0.004 strophantidine, 5 HEPES; $\mathrm{pH}$ adjusted to 7.2 with $\mathrm{CsOH}$

Pipette solution for persistent $\boldsymbol{I}_{N a}$ measurements (in $\mathbf{m M}$ ): $95 \mathrm{CsCl}, 40$ Cs-glutamate, 10 $\mathrm{NaCl}, 0.92 \mathrm{MgCl}_{2}, 1$ EGTA, $5 \mathrm{Mg}$-ATP, 0.3 Li-GTP, $0.36 \mathrm{CaCl}_{2}, 0.03$ niflumic acid, 0.02 nifedipine, 0.004 strophantidine, 5 HEPES; $\mathrm{pH}$ adjusted to 7.2 with $\mathrm{CsOH}$

External solution for $\boldsymbol{I}_{\boldsymbol{N a}}$ measurements (in mM): $5 \mathrm{NaCl}, 135 \mathrm{TMAC}, 4 \mathrm{CsCl}, 2 \mathrm{MgCl}_{2}$, $0.4 \mathrm{CaCl}_{2}, 10 \mathrm{D}(+)$-glucose, $10 \mathrm{HEPES}$; $\mathrm{pH}$ was adjusted to 7.4 with $\mathrm{CsOH}$

External solution for persistant $\boldsymbol{I}_{\boldsymbol{N a}}$ measurements (in mM): $135 \mathrm{NaCl}, 5 \mathrm{TMAC}, 4 \mathrm{CsCl}, 2$ $\mathrm{MgCl}_{2}, 0.4 \mathrm{CaCl}_{2}, 10 \mathrm{D}(+)$-glucose, $10 \mathrm{HEPES} ; \mathrm{pH}$ adjusted to 7.4 with $\mathrm{CsOH}$ 


\subsection{Methods}

\subsubsection{Cell culture}

All cells were cultivated under humidified conditions at $37{ }^{\circ} \mathrm{C}$ and $5 \%$ carbon dioxide (incubators from Heraeus Instruments or Thermo Scientific). All culture work was performed under sterile conditions using a laminar airflow cabinet (Heraeus Instruments or Thermo Scientific) to avoid microbiological contamination. Tissue culture dishes $(35 \mathrm{x} 10 \mathrm{~mm}, 60 \mathrm{x}$ $15 \mathrm{~mm}, 100$ x $20 \mathrm{~mm}$ ) and 12-well plates were bought from Starlab.

\subsubsection{Isolation and cultivation of human bone marrow-derived mesenchymal stem cells}

Bone marrow aspirates from iliac crest of a BrS patient and a healthy donor were diluted $1: 2-3$ in DMEM depending on the viscosity. In a $50 \mathrm{ml}$ reagent and centrifuge tube (Sarstedt), $20 \mathrm{ml}$ of the diluted bone marrow aspirate was carefully poured over $20 \mathrm{ml}$ lymphocyte separation medium 1077, a separation solution made with Ficoll ${ }^{\mathrm{TM}}$ density gradient media. After 15 min centrifugation at $800 \mathrm{x}$ g without brake (Eppendorf Centrifuge 5810R), mononuclear cells were obtained from the interphase between the plasma and the separation solution. The cells were washed two times with DMEM and plated onto $0.1 \% \mathrm{w} / \mathrm{v}$ gelatin coated tissue culture dishes in hMSC medium (see 2.1.5). The fibroblast-like hMSCs attached to the culture dish and could be separated from nonadherent cells by medium change. The medium was changed every second day and the cells were passaged every 3 to 4 days using $0.1 \%$ trypsin/EDTA. Single cells were resuspended in hMSC medium and seeded onto new culture dishes.

\subsubsection{Cultivation and inactivation of mouse embryonic fibroblasts}

MEFs were isolated from 15- to 17-day-old mouse embryos and subsequently cultivated on $0.1 \%$ gelatin coated culture dishes in feeder layer medium (see 2.1.5) for at most 4 passages. Before used for cultivation of hESCs or hiPSCs, MEFs were treated with a final concentration of $10 \mu \mathrm{g} / \mathrm{ml}$ mitomycin $\mathrm{C}$ for $3 \mathrm{~h}$ at $37{ }^{\circ} \mathrm{C}$ to stop cell proliferation. MEFs were washed three times with DPBS and subsequently treated with $0.2 \%$ trypsin until the adherent cells started to detach. The single cells were resuspended in feeder layer medium and counted with a Thoma counting cell chamber. A certain number of cells (e. g. 3 × $10^{5}$ cells on $6 \mathrm{~cm}$ dishes) was then plated onto $0.1 \%$ gelatin coated culture dishes. 


\subsubsection{Generation of human iPSCs}

Human iPSCs were generated by transducing hMSCs with the STEMCCA lentivirus containing four different transcription factors (see 2.1.4). One day before transduction, the hMSCs were plated onto $0.1 \%$ gelatin coated 12 -well plates with $0.3 \times 10^{5}$ cells per well. The cells were infected with STEMCCA particles at a multiplicity of infection (MOI) of 0.5, 1.0 and 2.0 in freshly prepared hMSC medium containing $1 \mu \mathrm{g} / \mathrm{ml}$ polybrene and $1 \mathrm{x}$ penicillinstreptomycin solution. Polybrene is a cationic polymer, which increases the efficiency of infection of eukaryotic cells with DNA. The next day medium was changed with fresh hMSC medium containing penicillin/streptomycin solution after washing the cells with DMEM twice. The medium was changed daily. From day 7 on the cells were cultivated on inactivated MEFs. The hMSC medium was replaced by hMSC medium mixed with hESC medium $(3: 1)$ at day 11 and by hES medium at day 14 after transduction. Single colonies that appeared with typical hESC morphology were picked mechanically using a sharpened glass pipette and were propagated further on MEFs (see 2.2.1.4).

\subsubsection{Cultivation of human iPSCs and ESCs with feeder cells}

In most experiments, undifferentiated hiPSCs and hESCs were cultivated on mitomycin Ctreated MEFs (see 2.2.1.2) in hESC medium. The medium was changed daily and cells were split every 4 to 5 days. The cells were treated with $200 \mathrm{U} / \mathrm{ml}$ collagenase type 4 for $5 \mathrm{~min}$ at $37{ }^{\circ} \mathrm{C}$ followed by two washing steps with DMEM/F12. The cells were additionally cut into small clusters using a cell scraper (Sarstedt). After pipetting two to three times up and down, the cells were seeded onto new culture dishes with inactivated MEFs. Colonies starting to differentiate were removed mechanically.

\subsubsection{Cultivation of human iPSCs without feeder cells}

In some experiments hiPSCs were cultivated on culture dishes coated with growth factor reduced Geltrex ${ }^{\mathrm{TM}}$ in feeder-free Essential $8^{\mathrm{TM}}$ medium. At about $85-90 \%$ confluency the cells were passaged onto new dishes. They were washed two times and incubated for 3 to 4 min with EDTA dissociation solution at room temperature. The small cell clusters or rather single cells were subsequently transferred in Essential $8^{\mathrm{TM}}$ medium onto new culture dishes coated with Geltrex ${ }^{\mathrm{TM}}$. After passaging, Pro-survival compound was added to the medium to a final concentration of $5 \mu \mathrm{M}$. The medium was changed daily. 


\subsubsection{Spontaneous differentiation of human iPSCs and ESCs in vitro}

For spontaneous in vitro differentiation experiments of hiPSCs and hESCs, the cells were treated with $200 \mathrm{U} / \mathrm{ml}$ collagenase type 4 (see 2.2.1.4) and dissected with a cell scraper into bigger clusters as compared to passaging. The cell clusters were transferred to bacteriological dishes (Sarstedt) and kept in suspension with hESC medium for 1 day. During this time, the cells formed multi-cellular aggregates known as embryoid bodies (EBs). After 1 day, the medium was changed to Iscove's medium and spent medium was changed every second day. At day 8, EBs were plated on $0.1 \%$ gelatin coated tissue culture dishes. During this spontaneous differentiation, clusters of beating CMs appeared beside other cell types. To circumvent cell overgrowth of noncardiac cells in long-term culture (3 months), FBS content in the differentiation medium was reduced from 20 to $5 \%$ after 1 month. Aditionally, beating clusters were picked and plated onto new gelatin coated culture dishes after 1 month and whenever necessary. After 1 and 3 months of maturation, the differentiated CMs were used for further experiments.

\subsubsection{Directed differentiation of human iPSCs into cardiomyocytes}

A protocol for directed cardiac differentiation as described previously (Lian et al. 2012; Lian et al. 2013) was used to obtain a high number of CMs resulting in a high amount of isolated cardiac-specific proteins for Western blot analyses. At about 95 - $100 \%$ confluency, the medium of undifferentiated hiPSCs cultivated under feeder-free conditions (see 2.2.1.5), was replaced by differentiation medium I supplemented with a final concentration of $9-10 \mu \mathrm{M}$

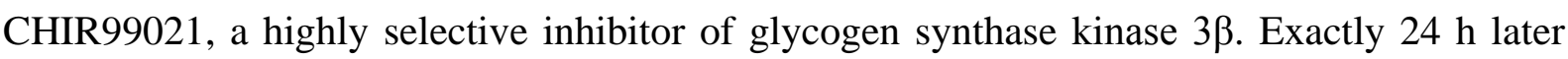
(=day 1), the medium was carefully replaced by differentiation medium I without CHIR99021. At day 3, half of the spent medium was aspirated and replaced with new differentiation medium I supplemented with a final concentration of $5 \mu \mathrm{M}$ Wnt antagonist II (IWP2). At day 5, the medium was replaced with fresh differentiation medium I without IWP2 and from day 7 on, the medium was replaced by differentiation medium II (see 2.1.5). First beating cells could be observed at day $7-8$ of differentiation. Between day 16 and 20, the medium was changed from differentiation medium II to cardiac selection medium to increase the yield of pure CMs. The cardiac selection medium lacks glucose, but contains lactate instead, which can be used as a source of energy only by CMs (Tohyama et al. 2013). After 4 to 5 days of selection, the medium was changed back to differentiation medium II and the selected CMs could be kept in culture for several months. 


\subsubsection{Freezing and thawing of cultivated cells}

For cryopreservation, hiPSCs and hESCs were treated with collagenase type 4 and dissected into small pieces as described before (see 2.2.1.4). The cell clusters were transferred into $15 \mathrm{ml}$ reagent and centrifuge tubes (Sarstedt) and centrifuged in DMEM/F12 at $180 \mathrm{x}$ g for 3 min. After centrifugation, the supernatant was discarded and the cells were carefully resuspended in $1 \mathrm{ml}$ freezing medium and transferred into cryovials (Greiner Bio-one). The cells were kept overnight at $-80{ }^{\circ} \mathrm{C}$ in a freezing box (Thermo Scientific) containing isopropanol, allowing controlled freezing at $-1{ }^{\circ} \mathrm{C}$ per min. The following day, the frozen cells were stored in liquid nitrogen.

For thawing, the frozen cells were placed in a $37{ }^{\circ} \mathrm{C}$ warm water bath. Immediately after thawing, the cells were transferred dropwise into $10 \mathrm{ml}$ DMEM/F12 and centrifuged at $180 \mathrm{x}$ g for $3 \mathrm{~min}$. The supernatant was discarded and the cell pellet was resupended in hESC or Essential $8^{\mathrm{TM}}$ medium and plated onto $6 \mathrm{~cm}$ culture dishes either coated with inactivated MEFs or with Geltrex ${ }^{\mathrm{TM}}$, respectively.

\subsubsection{Alkaline phosphatase staining}

One of the first evidence for successful somatic cell reprogramming is alkaline phosphatase activity. In reprogrammed cells, alkaline phosphatase expression is significantly increased. Alkaline phosphatase activity in hiPSCs was detected using an alkaline phosphatase staining kit according to the manufacturer's instructions. Cells were washed with DPBS and subsequently fixed for $30 \mathrm{~s}$ using a fixation solution provided by the kit. Fixed cells were washed two times with $\mathrm{dH}_{2} \mathrm{O}$ and stained for $15 \mathrm{~min}$ at $37{ }^{\circ} \mathrm{C}$ with a staining solution. After a final washing step with $\mathrm{dH}_{2} \mathrm{O}$, cells were dried at room temperature. Alkaline phosphatase positive cells are stained in red.

\subsubsection{Genomic DNA isolation for DNA sequencing}

Prior to genomic DNA isolation, cultivated cells (without MEFs) were treated with accutase for cell dissociation and washed with DPBS. After centrifugation, single cells were resuspended in $400 \mu \mathrm{l}$ DPBS. The genomic DNA was isolated and purified using the automated Maxwell ${ }^{\circledR} 16$ cell DNA purification kit according to the manufacturer's instructions. The concentration of the isolated DNA was measured with an Eppendorf biophotometer at $260 \mathrm{~nm}$ and $280 \mathrm{~nm}$. The samples were subsequently stored at $-80{ }^{\circ} \mathrm{C}$. For 
genomic DNA sequencing, the DNA sequence from the gene of interest (SCN5A) was initially amplified by PCR using the primer set SCN5A (1). In each reaction, $100 \mathrm{ng}$ of total genomic DNA was used. The PCR product was electrophoretically separated on a $1.5 \%$ agar gel and subsequently excised with a scalpel. The DNA fragment was then extracted from the agar gel using the QIAquick ${ }^{\circledR}$ gel extraction kit according to the manufacturer's instructions using a microcentrifuge. The DNA sequencing was performed by a commercial sequencing facility (Seqlab, Göttingen).

\subsubsection{Epigenetic analyses}

Cytosines in so-called "CpG sites", where a cytosine nucleotide occurs next to a guanine nucleotide, can be methylated by DNA methyltransferases to form 5-methylcytosine. This methylation of $\mathrm{CpG}$ sites within the promoter of a gene can lead to its transcriptional silencing. Bisulfite-treated DNA can be sequenced to determine the methylation status at $\mathrm{CpG}$ dinucleotides. This is possible because bisulphite treatment converts unmethylated cytosine residues into uracil, whereas 5-methylcytosines stay unaffected. Through direct comparison of the DNA sequence of interest before and after bisulfite treatment, one can distinguish between methylated and unmethylated cytosines. The methylation pattern of the promoter regions of the pluripotency related genes NANOG and OCT4 was compared before and after reprogramming of human MSCs into iPSCs. For this purpose, the genomic DNA of hMSCs, the generated hiPSCs between passage 8 and 16, and hESCs as positive control was isolated using the DNA purification from blood or body fluids spin protocol from the QIAamp ${ }^{\circledR}$ DNA mini kit according to the manufacturer's instructions.

Sodium bisulfite sequencing assays were performed by Epigenomics in Berlin. The region from -378 to +64 bp relative to the transcription start site of $N A N O G$ and -213 to +185 bp relative to the transcription start site of OCT4 were analyzed using the following primers:

NANOG:

5'-TAA TTT CAA ACT CCT AAC TTC AAA TAA T-3’and 5'-TAA TAT GAG GTA ATT AGT TTA GTT TAG T-3'

OCT4:

5'-GAT TTG TAT TGA GGT TTT GGA-3' and 5'-TCC AAA AAA ACC TTA AAA ACT T-3 


\subsubsection{Gene expression analyses}

\subsubsection{RNA isolation}

For gene expression analyses, cultured cells were washed with DPBS three times. Depending on the density, cells were lysed with $300-500 \mu 1$ RNA lysis buffer. Total RNA isolation and purification was done with the SV total RNA isolation system according to the manufacturer's instructions without the heating step in RNA dilution buffer. The concentration of the isolated RNA was measured with an Eppendorf biophotometer at $260 \mathrm{~nm}$ and $280 \mathrm{~nm}$. The RNA was subsequently used for reverse transcription (RT) reaction or stored at $-80{ }^{\circ} \mathrm{C}$.

\subsubsection{Reverse transcription reaction}

After RNA isolation, total mRNA was transcribed into complementary DNA (cDNA) using the enzyme reverse transcriptase. All components for one RT reaction are listed in Table 7.

Table 7. RT reaction components

\begin{tabular}{cr}
\hline \hline Components for RT & 20 $\boldsymbol{\mu l}$ final volume \\
\hline \hline 200 ng RNA + DEPC-treated $\mathrm{H}_{2} \mathrm{O}$ & $10.2 \mu \mathrm{l}$ \\
10x PCR buffer II & $2 \mu \mathrm{l}$ \\
$25 \mathrm{mM} \mathrm{MgCl}_{2}$ & $4 \mu \mathrm{l}$ \\
$100 \mathrm{mM}$ dNTPs & $0.8 \mu \mathrm{l}$ \\
RNase inhibitor $(20 \mathrm{U} / \mu \mathrm{l})$ & $1 \mu \mathrm{l}$ \\
$50 \mu \mathrm{M}$ Oligo d(T) $)_{16}$ & $1 \mu \mathrm{l}$ \\
\hline MuL V reverse transcriptase $(50 \mathrm{U} / \mu \mathrm{l})$ & $1 \mu \mathrm{l}$ \\
\hline \hline
\end{tabular}

The RT reaction was accomplished in a thermocycler (SensoQuest) using the following program:

$\begin{array}{lr}10 \text { min } & 22{ }^{\circ} \mathrm{C} \\ 50 \text { min } & 42{ }^{\circ} \mathrm{C} \\ 10 \text { min } & 95{ }^{\circ} \mathrm{C} \\ \infty & 4{ }^{\circ} \mathrm{C}\end{array}$

The resulting cDNA was stored at $-80^{\circ} \mathrm{C}$. 


\subsubsection{Semi-quantitative PCR}

All components for one PCR to amplify certain cDNA fragments are listed in Table 8.

Table 8. PCR reaction components

\begin{tabular}{|c|c|}
\hline Components for PCR & $50 \mu$ l final volume \\
\hline cDNA & $2 \mu 1$ \\
\hline DEPC-treated $\mathrm{H}_{2} \mathrm{O}$ & $30.6 \mu 1$ \\
\hline $5 x$ Green GoTaq ${ }^{\circledR}$ reaction buffer & $10 \mu \mathrm{l}$ \\
\hline $10 \mathrm{mM}$ dNTPs & $3.2 \mu 1$ \\
\hline sense primer $(10 \mu \mathrm{M})$ & $2 \mu 1$ \\
\hline antisense primer $(10 \mu \mathrm{M})$ & $2 \mu \mathrm{l}$ \\
\hline $\mathrm{GoTaq}^{\circledR}$ DNA polymerase & $0.2 \mu 1$ \\
\hline
\end{tabular}

The reaction was performed in a thermocycler using the following standardized program:

\begin{tabular}{ll|l}
$3 \mathrm{~min}$ & $95^{\circ} \mathrm{C}$ \\
$15 \mathrm{~s}$ & $95{ }^{\circ} \mathrm{C}$ & \\
$15 \mathrm{~s}$ & $55^{\circ} \mathrm{C}^{*}$ & $32-38$ cycles $^{*}$ \\
$30 \mathrm{~s}$ & $72{ }^{\circ} \mathrm{C}$ & \\
$10 \mathrm{~min}$ & $72{ }^{\circ} \mathrm{C}$ \\
$\infty$ & $4{ }^{\circ} \mathrm{C}$
\end{tabular}

*Exact annealing temperature and number of cycles for the different oligonucleotides are listed in Table 1.

\subsubsection{Gel electrophoresis}

All amplified PCR products were analyzed by electrophoretic separation on a $1.5 \%$ agar gel. The particular size of the DNA fragments was determined by GeneRuler ${ }^{\mathrm{TM}} 100 \mathrm{bp}$ Plus DNA Ladder. All results were documented under ultraviolet light with MultiImage Light Cabinet (Alpha Innotech Corporation). 


\subsubsection{Quantitative real-time PCR}

Quantitative real-time PCR (qPCR) was used to amplify cDNA products from the RT reaction for comparison of the cardiac-specific gene expression between BrS-CMs and Ctrl-CMs. All components for one qPCR are listed in Table 9.

Table 9. qPCR reaction components

\begin{tabular}{|c|c|}
\hline Components for qPCR & $10 \mu \mathrm{l}$ final volume \\
\hline cDNA & $1 \mu 1$ \\
\hline DEPC-treated $\mathrm{H}_{2} \mathrm{O}$ & $3.5 \mu 1$ \\
\hline SYBR $^{\circledR}$ Green PCR master mix & $5 \mu \mathrm{l}$ \\
\hline sense primer $(10 \mu \mathrm{M})$ & $0.25 \mu 1$ \\
\hline antisense primer $(10 \mu \mathrm{M})$ & $0.25 \mu \mathrm{l}$ \\
\hline
\end{tabular}

Triplicates for each sample were carefully added into the appropriate wells of a MicroAmp ${ }^{\circledR}$ optical 384-well reaction plate (Life Technologies ${ }^{\mathrm{TM}}$ ) and the plate was sealed with an adhesive film. After a brief centrifuge spin at $1000 \mathrm{rpm}$, the qPCR was performed in a 7900HT fast real-time PCR system (Life Technologies ${ }^{\mathrm{TM}}$ ) using the following program:

\begin{tabular}{ll|lll|l}
$10 \mathrm{~min}$ & $95{ }^{\circ} \mathrm{C}$ & followed by: & $15 \mathrm{~s}$ & $95^{\circ} \mathrm{C}$ & dissociation curve \\
$15 \mathrm{~s}$ & $95{ }^{\circ} \mathrm{C}$ & 40 cycles & $15 \mathrm{~s}$ & $60{ }^{\circ} \mathrm{C}$ & diss \\
$1 \mathrm{~min}$ & $60{ }^{\circ} \mathrm{C}$ & & $15 \mathrm{~s}$ & $95^{\circ} \mathrm{C}$ &
\end{tabular}

$\mathrm{SYBR}^{\circledR}$ Green is a fluorescent dye which intercalates with double-stranded DNA. Upon DNA amplification during qPCR, the fluorescence signal increases. This allows the quantification of the PCR product at any point in the amplification process by measuring the fluorescence. The SDS 2.4 software (Life Technologies ${ }^{\mathrm{TM}}$ ) was used for the operation of the cycler and analysis of the data. The software automatically determined a threshold for the detection of DNA-based fluorescence. The number of cycles at which the fluorescence crosses this threshold is called the threshold cycle $\left(\mathrm{C}_{\mathrm{t}}\right)$. During amplification, the fluorescence of a particular reaction will reach the threshold at an earlier cycle if the given gene is higher expressed in that sample compared to another sample. However, the qPCR efficiency is strongly dependent on the target gene and its corresponding primers. A relative standard curve for each pair of primers was generated by serial dilution of a cDNA sample to determine the 
particular efficiency. An efficiency of $100 \%$ means a doubling of the product with each cycle. The ratio of gene expression change $(\mathrm{R})$ of the samples relative to the controls was calculated using the following equations.

(1) $\Delta \mathrm{C}_{\mathrm{t}}=\mathrm{C}_{\mathrm{t}}$ (target gene $)-\mathrm{C}_{\mathrm{t}}($ reference gene $)$

(2) $\Delta \Delta \mathrm{C}_{\mathrm{t}}=\Delta \mathrm{C}_{\mathrm{t}}(\mathrm{BrS})-\Delta \mathrm{C}_{\mathrm{t}}(\mathrm{Ctrl})$

(3) $\mathrm{R}=2^{-\Delta \Delta \mathrm{Ct}}$

\subsubsection{Allele-specific expression analysis of the $S C N 5 A$ gene}

One allele of the SCN5A gene of the BrS patient is characterized by a point mutation in exon 28, where the cytosine is substituted by an adenine nucleotide (C5435A). For an allelespecific expression analysis of SCN5A, the mRNA of differentiated CMs from the BrS patient was sequenced using the Ion Torrent ${ }^{\mathrm{TM}}$ semiconductor sequencing system (Life Technologies $\left.{ }^{\mathrm{TM}}\right)$.

Initially, 3-month-old beating clusters from six independent spontaneous differentiation experiments and 2-month-old selected CMs from one directed differentiation experiment were collected and stored at $-80{ }^{\circ} \mathrm{C}$ until further use. The total amount of mRNA was isolated and reverse transcribed into cDNA as described before (see 2.2.5.1 and 2.2.5.2). A 189 bp long DNA fragment, which includes the region with the point mutation, was amplified using the primer set SCN5A (2), with Colorless GoTaq ${ }^{\circledR}$ reaction buffer and 1x Q-solution (Qiagen). The time for DNA denaturation and primer annealing was increased to $30 \mathrm{~s}$. The PCR product was diluted 1/200 and used for a second PCR run with 12 cycles using a forward primer and different reverse primers, which contained a unique barcode to distinguish different samples from different experiments (Table 10).

The DNA concentration of each sample was measured with Qubit $^{\circledR}$ 2.0 Fluorometer (Life Technologies ${ }^{\mathrm{TM}}$ ) and $250 \mathrm{ng}$ of each sample was pooled and electrophoretically separated on a $2 \%$ agar gel. The specific product was extracted from the gel in a QIAcube system (Qiagen) using the QIAquick ${ }^{\circledR}$ gel extraction kit according to the manufacturer's instructions. After gel extraction, the DNA was purified with Agencourt $^{\circledR}$ AMPure $^{\circledR}$ XP PCR purification kit according to the manufacturer's instructions and eluted in low TE buffer (Life Technologies $\left.{ }^{\mathrm{TM}}\right)$. 
Table 10. Primers with specific barcodes for Ion Torrent ${ }^{\mathrm{TM}}$ sequencing

\begin{tabular}{|c|c|}
\hline Name & Sequence \\
\hline SCN5A_BC01 & $\begin{array}{c}\text { 5'-CCA TCT CAT CCC T* }^{*} \text { G }^{*} \text { C GTG TCT CCG ACT CAG CTA AGG TAA CCG ATC } \\
\text { ACC ATG GGC AGG TCC ATG TTG ATG-3 }\end{array}$ \\
\hline SCN5A_BC02 & $\begin{array}{c}\text { 5’-CCA TCT CAT CCC T*G*C GTG TCT CCG ACT CAG TAA GGA GAA CCG } \\
\text { ATC ACC ATG GGC AGG TCC ATG TTG ATG-3’ }\end{array}$ \\
\hline SCN5A_BC03 & 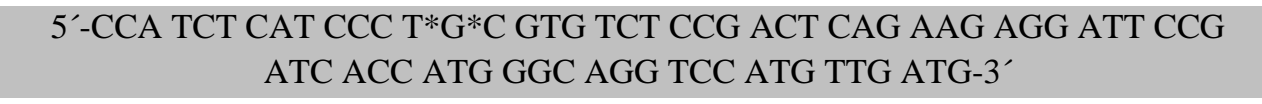 \\
\hline SCN5A_BCO4 & $\begin{array}{c}5^{\prime} \text {-CCA TCT CAT CCC T*G*C GTG TCT CCG ACT CAG TAC CAA GAT CCG ATC } \\
\text { ACC ATG GGC AGG TCC ATG TTG ATG-3' }\end{array}$ \\
\hline SCN5A_BC05 & $\begin{array}{c}\text { 5'-CCA TCT CAT CCC T*G*C GTG TCT CCG ACT CAG CAG AAG GAA CCG } \\
\text { ATG ACA GGG CAT CGG CAA AGT CAG ACA-3' }\end{array}$ \\
\hline SCN5A_BC06 & 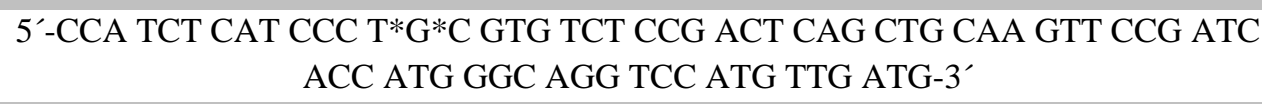 \\
\hline SCN5A_BC07 & $\begin{array}{l}5^{\prime} \text {-CCA TCT CAT CCC T* } \mathrm{G}^{*} \mathrm{C} \text { GTG TCT CCG ACT CAG TTC GTG ATT CCG ATC } \\
\text { ACC ATG GGC AGG TCC ATG TTG ATG-3' }\end{array}$ \\
\hline SCN5A_BC08 & $\begin{array}{c}5^{\prime} \text {-CCA TCT CAT CCC } \mathrm{T}^{*} \mathrm{G}^{*} \mathrm{C} \text { GTG TCT CCG ACT CAG TTC CGA TAA CCG ATG } \\
\text { ACA GGG CAT CGG CAA AGT CAG ACA-3' }\end{array}$ \\
\hline SCN5A_BC09 & $\begin{array}{c}5^{\prime} \text {-CCA TCT CAT CCC T*G*C GTG TCT CCG ACT CAG TGA GCG GAA CCG } \\
\text { ATC ACC ATG GGC AGG TCC ATG TTG ATG-3' }\end{array}$ \\
\hline SCN5A_for & 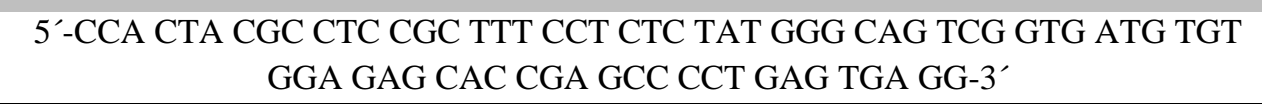 \\
\hline
\end{tabular}

The DNA quantity was determined by qPCR analysis in a 384-well plate using the GeneRead Library Quant kit. The purified PCR product was diluted 1/10 000 and 1/100 000. One reaction mix contained $3.86 \mu$ l RNAse free $\mathrm{H}_{2} \mathrm{O}, 5.68 \mu \mathrm{l}$ GeneRead qPCR SYBR ${ }^{\circledR}$ Green mastermix, $0.45 \mu 1$ primer mix and $3 \mu \mathrm{l}$ of the template. The PCR reactions of the DNA standards and sample dilutions were performed in triplicates in a 7900HT fast real-time PCR system using the following program:

\begin{tabular}{|c|c|}
\hline $2 \mathrm{~min}$ & $50^{\circ} \mathrm{C}$ \\
\hline $10 \mathrm{~min}$ & $95^{\circ} \mathrm{C}$ \\
\hline $15 \mathrm{~s}$ & $95^{\circ} \mathrm{C}$ \\
\hline $1 \mathrm{~min}$ & $60^{\circ} \mathrm{C}$ \\
\hline
\end{tabular}

$400 \mu \mathrm{l}$ of $10 \mathrm{pM}$ PCR product was used for clonal amplification onto Ion Sphere ${ }^{\mathrm{TM}}$ particles accomplished by an emulsion PCR in the Ion OneTouch ${ }^{\mathrm{TM}}$ (Life Technologies ${ }^{\mathrm{TM}}$ ) system according to the manufacturer's instructions. The Ion Sphere ${ }^{\mathrm{TM}}$ particles coated with the amplified template DNA were applied to an Ion Torrent ${ }^{\mathrm{TM}}$ sequencing chip and placed on the Ion Personal Genome Machine (PGM ${ }^{\mathrm{TM}}$ ) for sequencing. The sequencing was kindly performed by the Clinical Pharmacology, University Medical Center Göttingen. 


\subsubsection{Western blot analyses}

\subsubsection{Preparation of cell lysates}

For protein analyses, CMs were generated from hiPSCs using the directed differentiation protocol including the cardiac selection to obtain a sufficient amount of cardiac cells (see 2.2.1.7). Prior to the preparation of the cell lysates, the 2-month-old CMs were washed with DPBS. After centrifugation and discarding the DPBS, the cell pellets were snap-frozen and stored at $-80{ }^{\circ} \mathrm{C}$ until further use.

The frozen cell pellets were lysed in $200 \mu \mathrm{l}$ cell lysis buffer by vortexing the samples and using an Omnican 40 syringe (B. Braun) for mechanical disruption. The samples were kept on ice to prevent enzymatic damage and centrifuged for 10 min at $4{ }^{\circ} \mathrm{C}$ to get rid of bigger cell fractions. The supernatant was transferred into new Eppendorf cups and $5 \mu$ of each sample was diluted in $95 \mu \mathrm{l}$ DPBS for protein measurements. The protein concentration was determined using the Pierce ${ }^{\mathrm{TM}} \mathrm{BCA}$ protein assay kit according to the manufacturer's instructions and a photometer at $562 \mathrm{~nm}$ (Biotek). The samples were mixed with blue loading buffer and the proteins were denatured at $70{ }^{\circ} \mathrm{C}$ for $10 \mathrm{~min}$ and stored at $-20{ }^{\circ} \mathrm{C}$ until further use.

\subsubsection{SDS-polyacrylamide gel electrophoresis}

Frozen cell lysate samples were thawed on ice. The proteins in the samples were separated by SDS-polyacrylamide gel electrophoresis (SDS-PAGE) according to their molecular weight. The gel electrophoresis was repeated 3 times on different gels. The samples were loaded into the wells of the stacking gel, followed by a separation gel with a polyacrylamid concentration of $7.5 \%$. One electrophoresis run for two different gels at the same time took nearly $2 \mathrm{~h}$ at 60 $\mathrm{mA}$. Both gels were covered by $1 \mathrm{x}$ running buffer all the time.

\subsubsection{Protein transfer and detection}

After electrophoretic separation, the proteins were electrically transferred in a blotting chamber (Bio-Rad) onto a nitrocellulose membrane (GE Healthcare) for antibody detection. The blotting chamber was filled with $1 \mathrm{x}$ transfer buffer and continuously cooled with ice during the electroblotting at $400 \mathrm{~mA}$ for $2 \mathrm{~h}$. 
The successful transfer of the proteins was confirmed by Ponceau S solution staining. The membrane was then washed three times with TBS-T buffer and subsequently blocked with $5 \%$ nonfat dry milk in TBS-T for $1 \mathrm{~h}$ at room temperature to avoid nonspecific binding. The primary antibodies were diluted in $5 \%$ nonfat dry milk in TBS-T (Table 2) and incubated with the membrane at $4{ }^{\circ} \mathrm{C}$ overnight with gentle agitation. The next day, the membrane was washed three times with TBS-T buffer for $10 \mathrm{~min}$ each, followed by a second incubation with horseradish peroxidase (HRP)-coupled secondary antibodies (Table 3) for $1 \mathrm{~h}$ at room temperature under gentle agitation. The membrane was again washed three times with TBS-T buffer. Visualization of the HRP-coupled secondary antibodies was done using Immobilon ${ }^{\mathrm{TM}}$ Western chemiluminescent HRP substrate. The HRP catalyzes the oxidation of luminol by peroxide resulting in luminescence. A light-sensitive photographic film (Fujifilm) was placed against the membrane, and du to the exposure of light bands representing proteins of interest could be captured on the film.

All data generated by Western Blot experiments were quantified using AlphaEase ${ }^{\mathrm{TM}}$ (Alpha Innotech) and GraphPad Prism ${ }^{\circledR} 5.02$ (GraphPad Software, Inc.) softwares.

\subsubsection{Immunofluorescence analyses}

The expression of pluripotency related proteins in undifferentiated hiPSCs, germ layerspecific proteins in differentiated hiPSCs, as well as cardiac-specific proteins in CMs was shown by immunostaining. The hiPSCs and differentiated cells were initially cultivated on cover slips (Thermo Scientific). Beating bodies were digested and the resulting single CMs were plated on $35 \mathrm{~mm}$ cell culture dishes with glass bottom (WPI) and further cultivated for at least 10 days before fixation. The cells were washed with DPBS two times, followed by 20 min fixation in $4 \%$ paraformaldehyde at room temperature with three further washing steps. The cells which were stained for nuclear transcription factors (OCT4, SOX2, and NANOG) as well as the cardiac-specific proteins cTNT, $\alpha$-actinin, Cx43, and MLC2a were additionally treated with $0.1 \%$ Triton $^{\mathrm{TM}} \mathrm{X}-100 / \mathrm{DPBS}$ for $10 \mathrm{~min}$ at room temperature. Prior to antibody incubation, the fixed cells were blocked in $1 \% \mathrm{BSA}$ overnight at $4{ }^{\circ} \mathrm{C}$. The cells were incubated with primary and subsequently with secondary antibodies, both diluted in $1 \%$ BSA (Table 2) for $1 \mathrm{~h}$ at $37{ }^{\circ} \mathrm{C}$ (NANOG at $4{ }^{\circ} \mathrm{C}$ overnight). The nuclei were stained with $0.4 \mu \mathrm{g} / \mathrm{ml}$ DAPI. The stained cells were washed with $\mathrm{dH}_{2} \mathrm{O}$ once before mounting with Fluoromount-G ${ }^{\mathrm{TM}}$. Fluorescent images were taken with a fluorescence microscope (Zeiss Observer.Z1 or Axio Imager.M2). For some images, the ApoTome modus (Zeiss) was used. 


\subsubsection{Teratoma formation and analysis}

Undifferentiated hiPSCs were mechanically dissected using a cell scraper and transferred into $200-300 \mu \mathrm{l}$ DPBS. The cell clusters were injected subcutaneously into recombination activating gene 2 and gamma $C$ deficient (RAGC) mice, which lack B cells, $\mathrm{T}$ cells, and natural killer cells. Teratomas were collected about three months after injection and fixed in phosphate buffered formalin ( $\mathrm{pH}$ 7.0) for $4 \mathrm{~h}$ at room temperature or at $4{ }^{\circ} \mathrm{C}$ overnight. After washing with $\mathrm{dH}_{2} \mathrm{O}$ the teratomas were dehydrated and paraffinized using the Benchtop Tissue Processor 1020 (Leica Biosystems). The samples were embedded into paraffin using a tissue embedding system (Leica Biosystems) and processed into $6 \mu \mathrm{m}$ sections with a microtome (Leica Biosystems). The histological sections were stained with hematoxylin and eosin at the Department of Pathology of the University Medical Center Göttingen and subsequently analyzed under a light microscope (Zeiss).

\subsubsection{Karyotyping}

All hiPSCs were cultured on Geltrex ${ }^{\mathrm{TM}}$ prior to analyzing the karyotypes. The cells were treated with $100 \mathrm{ng} / \mathrm{ml}$ of the microtubule-depolymerizing drug colcemid (Karyomax ${ }^{\circledR}$ Colcemid $^{\circledR}$ solution) for $16 \mathrm{~h}$. The supernatant containing detached cells was collected in a $15 \mathrm{ml}$ reagent and centrifuge tube and the adherent cells were washed with DMEM basal medium once and subsequently treated with TrypLETM for 1 min at $37^{\circ} \mathrm{C}$. Single cells were collected in the $15 \mathrm{ml}$ tube and centrifuged at $200 \mathrm{x}$ g for $5 \mathrm{~min}$. The supernatant was discarded, leaving about $0.5 \mathrm{ml}$ in which the cell pellet was resupended by tapping carefully against the tube. Pre-warmed $\left(37^{\circ} \mathrm{C}\right) \mathrm{KCl}$ solution $(0.075 \mathrm{M})$ was added drop-wise to the cell suspension up to $8 \mathrm{ml}$ while shaking the tube carefully. After $45 \mathrm{~min}$ of incubation at $37{ }^{\circ} \mathrm{C}$, the cells were centrifuged and the supernatant was aspirated to a residue of $0.5 \mathrm{ml}$, in which the cells were resupended again. Freshly prepared and pre-cooled $\left(-20^{\circ} \mathrm{C}\right)$ fixation buffer was then added drop-wise to the suspension while shaking the tube carefully. The cells were incubated on ice for $10 \mathrm{~min}$ and then centrifuged. The fixation step was repeated two times. After the third fixation, the cells were resuspended in a final volume of about $2 \mathrm{ml}$ to obtain an optimal density before dropping them onto cold microscope slides. After air-drying, the chromosomes were stained with Giemsa solution $\left(1 / 20\right.$ diluted in $\left.\mathrm{dH}_{2} \mathrm{O}\right)$ for $5 \mathrm{~min}$ and washed twice with tap water. The stained chromosomes were counted under a light microscope (Zeiss Axio Imager.M2) and documented using the karyotyping analysis software Case Data Manager 6.0 (Applied Spectral Imaging, ASI). 


\subsubsection{Electrophysiological analyses}

\subsubsection{Cardiomyocyte preparation and data acquisition}

Single beating CMs from spontaneous differentiation experiments were used at early (1 month) and late (3 months) developmental stages. Beating areas were picked mechanically using a sharpened glass pipette and either digested with collagenase type 2 and 4 (1:1) for up to $1 \mathrm{~h}$ or with $0.25 \%$ trypsin/EDTA solution for $25-30 \mathrm{~min}$ at $37^{\circ} \mathrm{C}$. The single $\mathrm{CMs}$ were plated onto $0.1 \%$ gelatin coated $3 \mathrm{~cm}$ tissue culture dishes and cultured in Iscove's medium with $5 \%$ FBS for at least a further 6 days for AP and $I_{N a}$ measurements. CMs were identified by cell contraction.

All electrophysiological recordings were performed at room temperature using an EPC10 USB patch clamp amplifier together with the PatchMaster software (HEKA). The signals were filtered with 2.9 and $10 \mathrm{kHz}$ Bessel filters.

\subsubsection{Action potential measurements}

APs were measured with the ruptured-patch whole-cell current clamp technique. Spontaneous APs were recorded in IMDM basal medium immediately after rupturing with self-pulled microeletrodes of $3-5 \mathrm{M} \Omega$ resistance (thin-wall capillaries, WPI). No current was injected into the cells. In some experiments, CMs were paced with $0.4,0.8$, and $1.0 \mathrm{~Hz}$. APs were analyzed using LabChart ${ }^{\circledR} 8$ Pro software (ADInstruments) to determine the RMP, the maximal upstroke velocity $\left(\mathrm{V}_{\max }\right)$, the AP amplitude (APA), the APDs at $50 \%, 70 \%$, and $90 \%$ of repolarization (APD50, APD70, APD90). The parameters of at least 10 APs in a row were averaged for the analysis.

\subsubsection{3 $I_{N a}$ measurements}

$I_{N a}$ was measured using the ruptured-patch whole-cell voltage clamp technique with selfpulled microelectrodes of $1.8-3 \mathrm{M} \Omega$ resistance. External solutions with low $\left[\mathrm{Na}^{+}\right]_{\mathrm{o}}(5 \mathrm{mM})$ were used for a better voltage control in peak current and gating property measurements. For persistent $I_{N a}$ measurements, an external solution with high $\left[\mathrm{Na}^{+}\right]_{\mathrm{o}}(135 \mathrm{mM})$ was used. Liquid junction potentials were corrected before cell attachment. All recordings started at least $1 \mathrm{~min}$ after rupture. Membrane capacitance $\left(C_{m}\right)$ and series resistance $\left(R_{s}\right)$ were compensated 
automatically after rupture. Measurements were performed using a holding potential of $-100 \mathrm{mV}$.

Current-voltage (I-V) relationship was determined by increasing the voltage stepwise from $-95 \mathrm{mV}$ to $+35 \mathrm{mV}$ in $5 \mathrm{mV}$ steps from a holding potential of $-100 \mathrm{mV}$. Each pulse lasted $50 \mathrm{~ms}$. All currents were normalized to $\mathrm{C}_{\mathrm{m}}$. Steady-state inactivation was measured by a double pulse protocol from a holding potential of $-120 \mathrm{mV}$ consisting of a $500 \mathrm{~ms}$ pulse of increasing amplitude from $-120 \mathrm{mV}$ to $-20 \mathrm{mV}$ in $5 \mathrm{mV}$ steps. $I_{N a}$ was normalized to the maximum current (which was usually after the first pulse). Steady-state activation and inactivation curves were fitted with a standard Boltzmann function:

$\mathrm{Y}=1 /\left(1+\exp \left(\left(\mathrm{V}_{1 / 2}-\mathrm{V}\right) / \kappa\right)\right)$, where $\mathrm{V}$ is the voltage, $\mathrm{V}_{1 / 2}$ is the half-maximal voltage of steady-state (in)activation and $\kappa$ is the slope factor of voltage dependence of (in)activation.

Intermediate inactivation of the sodium channels was measured by a double pulse protocol from a holding potential of $-100 \mathrm{mV}$ to $-20 \mathrm{mV}$. The duration of the first pulse increased stepwise starting with $15 \mathrm{~ms}$ up to $1054 \mathrm{~ms}$. After the first pulse, a recovery interval at $-100 \mathrm{mV}$ for $20 \mathrm{~ms}$ was used to recover all sodium channels that were not in the intermediate inactivation state. The second test pulse at $-20 \mathrm{mV}$ was used to measure the $I_{N a}$ generated by the noninactivated sodium channels. The peak current from the second pulse was divided by the first pulse and then normalized to the $15 \mathrm{~ms}$ peak current. The development of intermediate inactivation was fitted with a single exponential function (one-phase decay):

$\mathrm{Y}(\mathrm{t})=\mathrm{Y}_{0}+\mathrm{A}(-1+\exp (-\mathrm{t} / \tau))$, where $\mathrm{Y}_{0}$ is the $\mathrm{Y}$ value at $\mathrm{t}=0, \mathrm{~A}$ is the amplitude, and $\tau$ the time constant of inactivation.

For the recovery from inactivation kinetics, a two-pulse protocol was used, with an increasing delay between the two pulses ranging from $1 \mathrm{~ms}$ to $165 \mathrm{~ms}$. The first conditioning pulse of $1000 \mathrm{~ms}$ duration induced sodium channel inactivation and the second pulse measured $I_{N a}$ generated by sodium channels that had recovered from inactivation. The data obtained from the second pulse were normalized to the current from the first pulse and fitted with a single exponential function (one-phase association):

$\mathrm{Y}(\mathrm{t})=\mathrm{Y}_{0}+\mathrm{A}\left(1-\exp \left(-\mathrm{t} / \tau_{\text {rec }}\right)\right)$, where $\mathrm{Y}_{0}$ is the $\mathrm{Y}$ value at $\mathrm{t}=0, \mathrm{~A}$ is the amplitude, and $\tau_{\text {rec }}$ the time constant of recovery from inactivation. 
Persistent $I_{N a}$ density was measured in the interval between $50-450 \mathrm{~ms}$ of a $1000 \mathrm{~ms}$ pulse from a holding potential of $-100 \mathrm{mV}$ to $-20 \mathrm{mV}$. Currents were normalized to $\mathrm{C}_{\mathrm{m}}$.

The data were analyzed using Clampfit (Axon Instruments), Excel (Microsoft), and GraphPad Prism ${ }^{\circledR} 5.02$ software.

\subsubsection{Statistical analyses}

Experimental data obtained from qPCR, Western blot, and patch clamp analyses are presented as mean \pm standard error of mean (SEM). For comparison of two data sets, two-tailed unpaired Student's $t$-test was applied. For comparison of more than two variables, the twoway repeated analysis of variance (ANOVA) test with Bonferroni posttests was applied. Statistical significance is expressed by the p-value represented as $\left(^{*}\right) \mathrm{p}<0.05,(* *) \mathrm{p}<0.01$, and $(\S) \mathrm{p}<0.001$. All statistical analyses were performed using GraphPad Prism ${ }^{\circledR} 5.02$ software. 


\section{Results}

\subsection{Clinical profile of a patient suffering from Brugada syndrome}

A 50-year-old male patient diagnosed with $\mathrm{BrS}$ was recruited in this study. The patient suffered from several seizures during his childhood and adolescence, including epileptic shocks. These symptoms, together with recurrent syncope, were first believed to have a neurological cause. However, findings in ECG recordings revealed a slight coved type STsegment elevation in the right precordial leads $\mathrm{V}_{1}$ and $\mathrm{V}_{2}$ (BrS type I ECG) and a more saddleback pattern in $\mathrm{V}_{3}$ (BrS type II ECG) followed by a negative T-wave (Fig. 7, arrows). Additionally, atrial fibrillation occurred spontaneously in rest (data not shown). In long-term ECG recordings multiple episodes of polymorphic ventricular tachycardia as well as ventricular extrasystoles appeared spontaneously. First-degree AV blocks could be induced, which are characterized by a delayed electrical conduction from the atria to the ventricles through the AV node. As a result a cardioverter-defibrillator was implanted to help reducing the number of arrhythmias experienced by the patient.
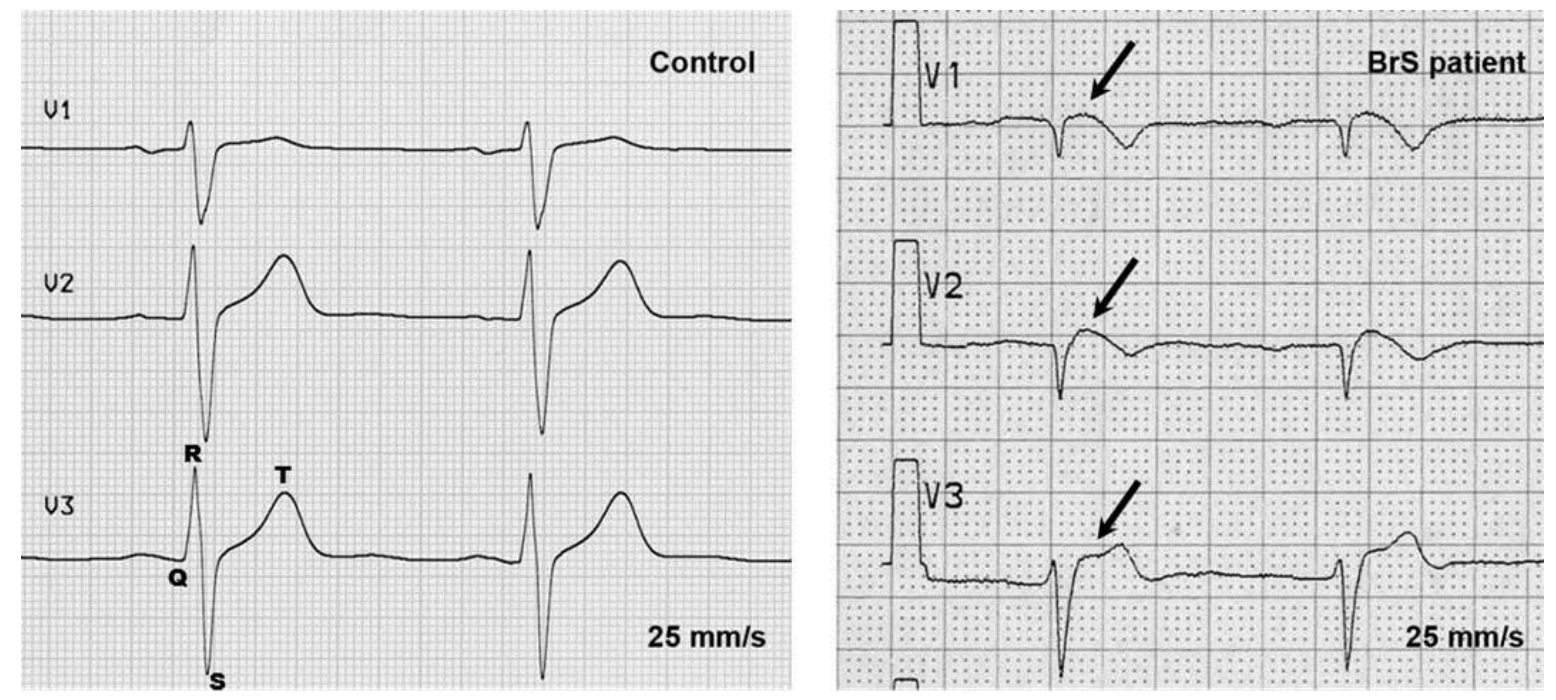

Figure 7. Resting ECGs from the BrS patient (right) and a 31-year-old healthy control (left). Recordings from precordial leads $\mathrm{V}_{1}-\mathrm{V}_{3}$ are shown. The recordings of the BrS patient show a slight coved ST-segment elevation in $\mathrm{V}_{1}$ and $\mathrm{V}_{2}$ (BrS type I ECG) and a more saddleback pattern in $\mathrm{V}_{3}$ (BrS type II ECG), followed by a negative T-wave in each case. ECG recordings were kindly provided by the University Medical Center Göttingen.

Genetic screening for possible mutations in several cardiac-specific genes showed that the patient is heterozygous for a nonsense mutation of the SCN5A gene. A nucleotide substitution in position 5435 of exon $28(\mathrm{C}>\mathrm{A})$ results in an in-frame premature termination codon (PTC). This mutation might lead to a truncation of Nav1.5 in the C-terminus (p.S1812X) and 
is associated with BrS (Schulze-Bahr et al. 2003; Ruan et al. 2009). The cardiac phenotype of the patient seems to have a familial background (Fig. 8). The point mutation in the SCN5A gene was also identified in some of the family members.

II

I

III

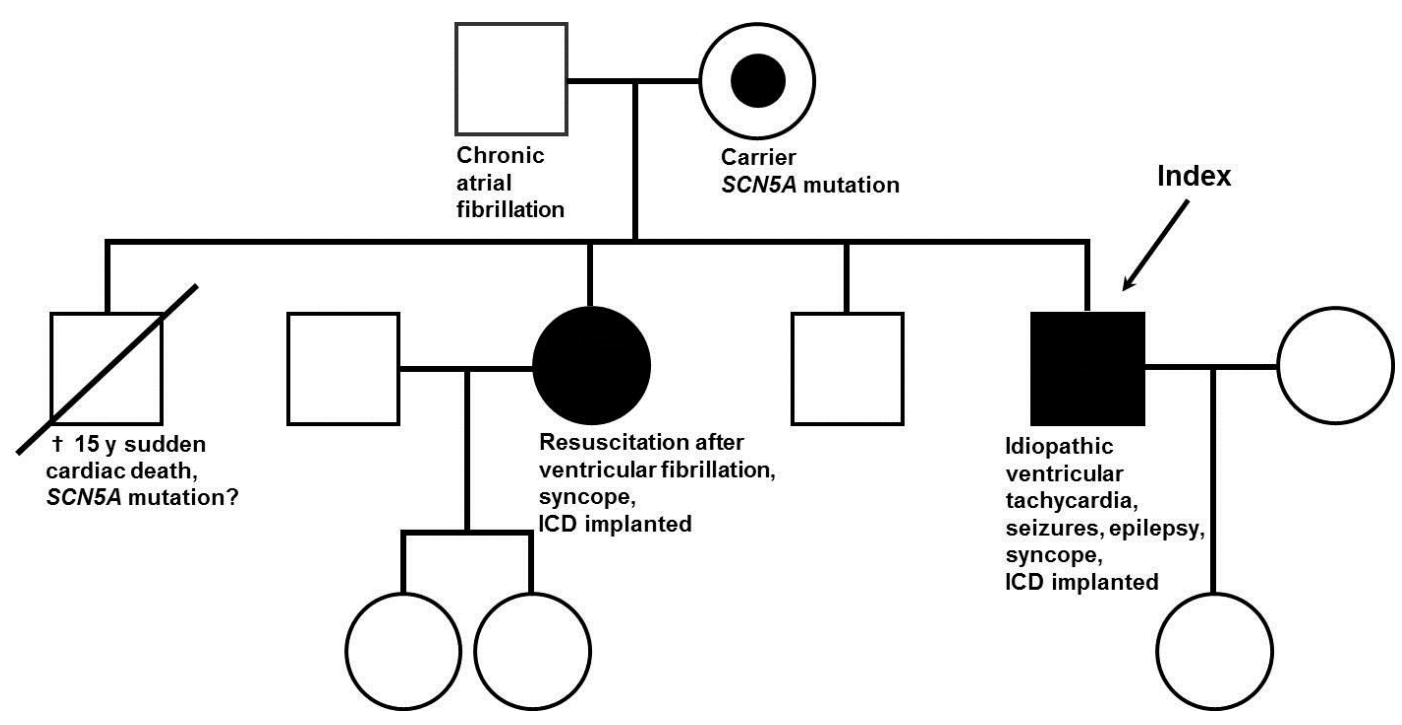

Figure 8. Family tree of the BrS patient. The 50-year-old BrS patient suffers from idiopathic ventricular tachycardia, several seizures and syncopes. He is heterozygous for a point mutation in the SCN5A gene, which leads to a PTC in exon 28. The patient carries an implantable cardioverter-defibrillator (ICD). His sister carries the mutation and had to be resuscitated after a collapse due to ventricular fibrillation. She also carries an ICD. Both have healthy daughters without this mutation. One brother died from sudden cardiac death at the age of 15. Genomic information is not known. A second brother is healthy and not carrier of the mutation. The father suffers from cardiac disorder, but is not carrier of the mutation, whereas the mother is carrier, but healthy.

One younger brother of the patient had to be resuscitated after a collapse due to physical stress at the age of 15. In the hospital, ventricular fibrillation was observed. He died one week later from sudden cardiac death. The following autopsy revealed an extensive fibrosis in the endocardium along the conduction system next to the left ventricle. There is no information regarding his genotype.

The sister had to be resuscitated after a collapse at the age of 25 years. She was found to carry the mutation. ECG patterns showed a discrete ST-segment elevation in the right precordial leads, but no BrS-specific alterations during rest. Idiopathic ventricular fibrillation was observed during flecainide medication which might be an indication of BrS. She also carries an implantable cardioverter-defibrillator.

A second brother of the index patient is healthy without the $S C N 5 A$ mutation. Two daughters of the sister and the daughter of the index patient neither carry the point mutation nor show any cardiac disorders so far. 
The mother carries the mutation, but is healthy and does not show a BrS-specific ECG pattern at rest. However, a first-degree AV block was also observed.

The father does not have the SCN5A mutation and does not show a BrS-specific ECG pattern. But he demonstrated atrial fibrillation, right ventricular arrhythmia, and ventricular extrasystoles in ECG recordings since the age of 52. The subject is obese.

\subsection{Generation of human iPSCs and proof of pluripotency}

\subsubsection{Generation of human iPSCs}

Bone marrow aspirate from iliac crest of the male $\mathrm{BrS}$ patient was taken by physicians at the University Medical Center Göttingen. As it was not possible to obtain cells from healthy realtives the bone marrow aspirate left-over from diagnosis of an unrelated healthy female person without known cardiac disease was used as control. The cell separation of the bone marrow samples was accomplished by the Ficoll gradient centrifugation. Mononuclear cells were collected from the interface after centrifugation. The hMSCs were isolated by their property to adhere to tissue culture dishes. Nine days after isolation about $2 \times 10^{6} \mathrm{hMSCs}$ with a fibroblast-like morphology (Fig. 10A, D) were obtained for further expansion.

The isolated hMSCs from the BrS patient and the control were transduced with STEMCCA lentivirus particles between passage 2 and 4 . The reprogramming procedure is illustrated in Fig. 9.

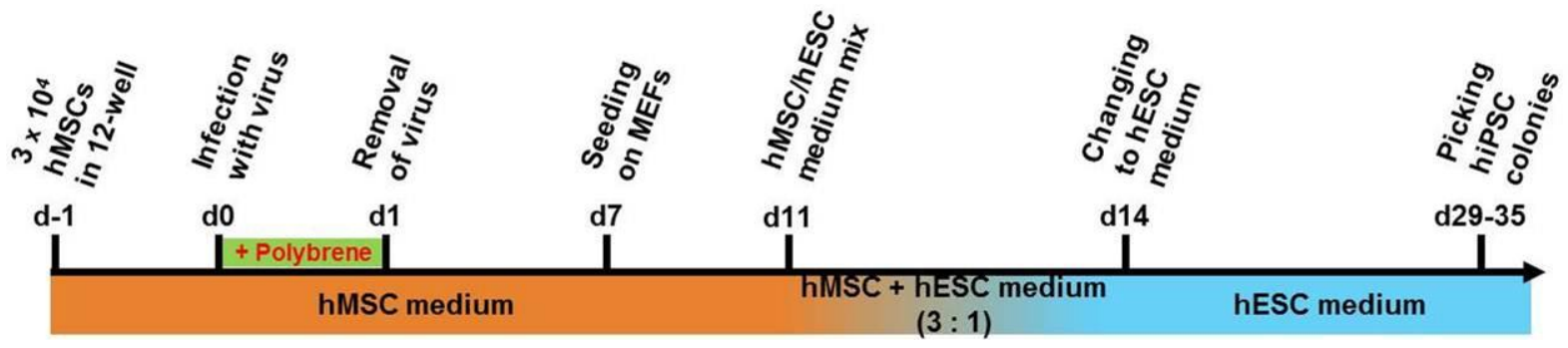

Figure 9. Scheme of reprogramming procedure of isolated hMSCs.

First colony-like areas appeared on day 11 after transduction. These cells were already positive for alkaline phosphatase (data not shown). Several colonies resembling hESCs with respect to their morphology were picked mechanically and propagated further on new culture dishes coated with feeder cells (Fig. 10B, E). Three independent cell clones from the BrS patient $(\mathrm{Na} 6-8)$ and the healthy donor (iBM76.1 - 3) were further characterized. The enzyme alkaline phosphatase was expressed in all generated hiPSCs (Fig. 10C, F). 

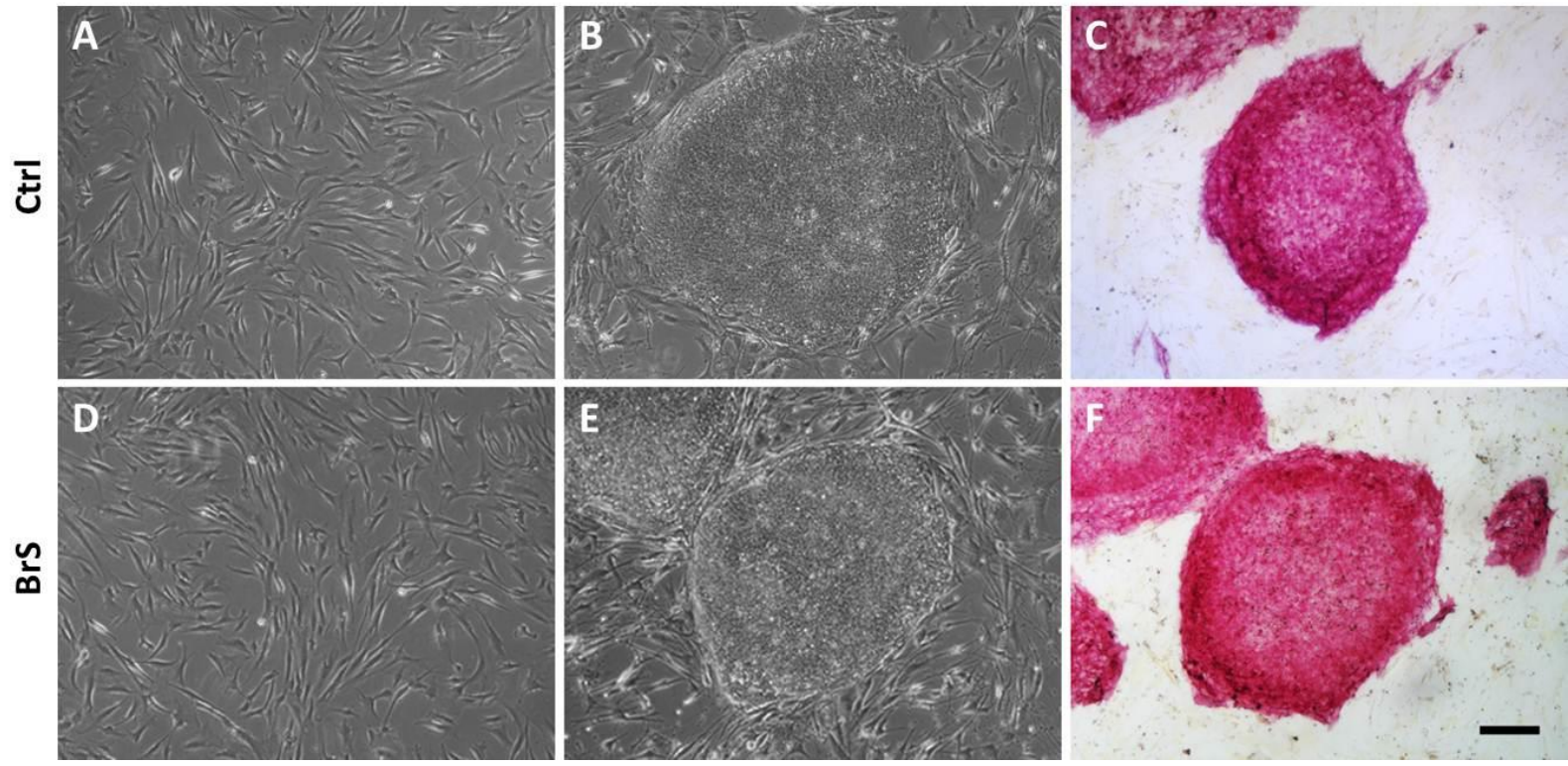

Figure 10. Generation of hiPSCs. Isolated and cultivated hMSCs from the bone marrow of the BrS patient (D) and the healthy donor as control (A) were reprogrammed into hiPSCs (B, E) using the STEMCCA lentivirus. The hiPSC clones showed alkaline phosphatase activity (C, F). Scale bar: $200 \mu \mathrm{m}$.

\subsubsection{Expression of pluripotency related markers}

Cellular reprogramming involves an extensive remodelling of gene expression. The expression of the pluripotency related marker genes NANOG, LIN28, GDF3, and FOXD3 was investigated by semiquantitative RT-PCR analyses in the generated hiPSCs compared to their parental hMSCs (BM76 and BM77). The results revealed a significant upregulation of these genes in all generated hiPSC clones (iBM76.1 - 3 and Na6 - 8), which was comparable to the expression level in hESCs (Fig. 11).

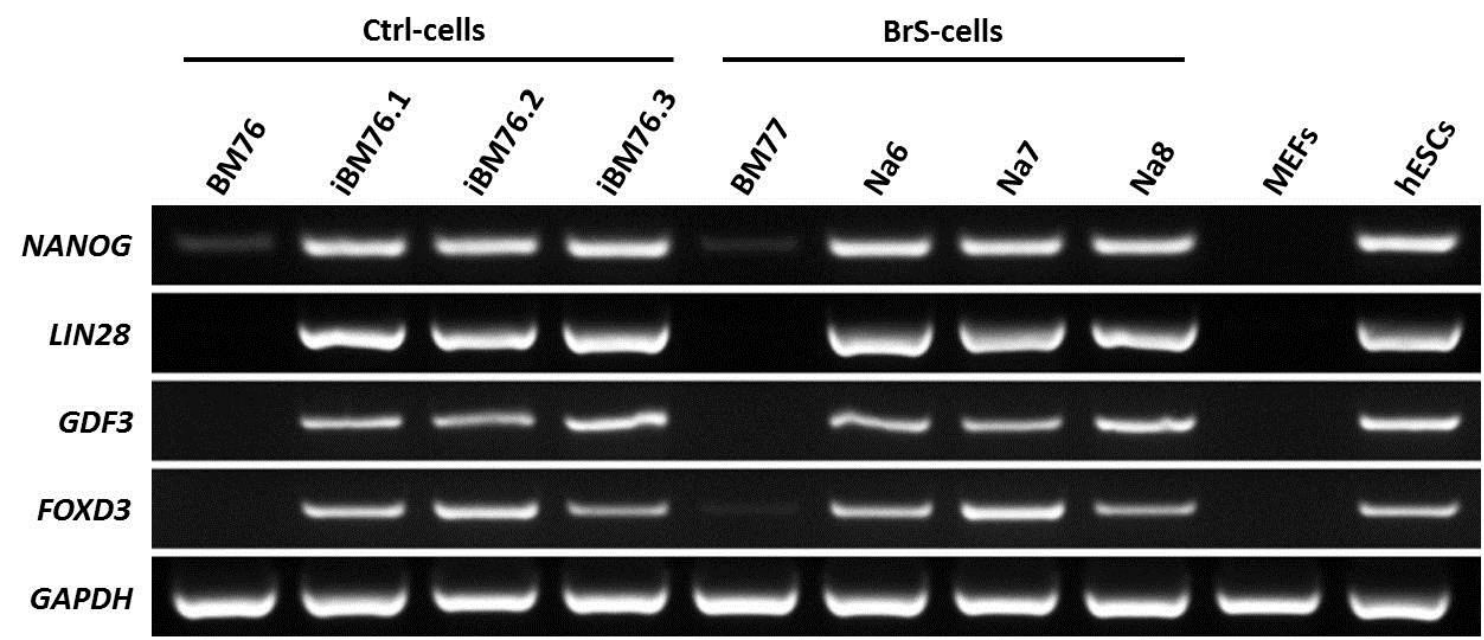

Figure 11. Gene expression analysis of generated hiPSCs and their parental hMSCs. The pluripotency related genes $N A N O G, L I N 28, G D F 3$, and FOXD3 were highly expressed in hESCs and in the generated hiPSCs. These genes were downregulated in the parental hMSCs (BM67 and BM77) as well as in MEFs. 
In contrast to $L I N 28$ and $G D F 3$, a very weak transcription of $N A N O G$ and FOXD3 was already detectable in the hMSCs. But their expression levels in the generated hiPSCs were much higher and comparable to that of pluripotent hESCs. These data show an activation of endogenous gene expression since the four genes are not component parts of the STEMCCA lentivirus vector.

In addition, the pluripotency related proteins NANOG, OCT4, SOX2, LIN28, SSEA4, and TRA-1-60 were detected in all generated hiPSC clones (Fig. 12).
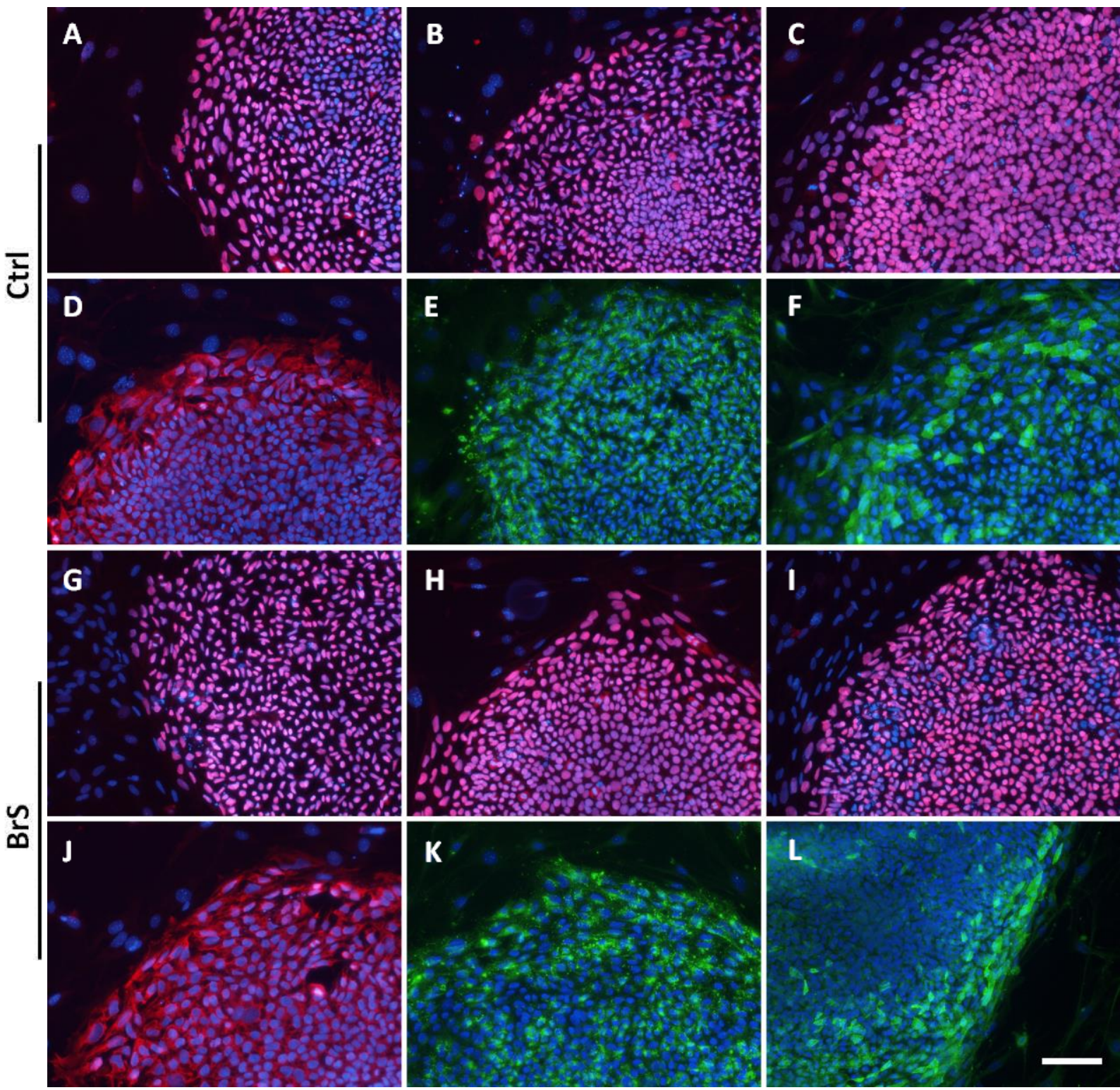

Figure 12. Immunostaining of the generated hiPSCs detecting pluripotency related proteins. Ctrl- $(\mathrm{A}-\mathbf{F})$ and BrS-hiPSCs $(\mathbf{G}-\mathbf{L})$ were positive for the transcription factors NANOG $(\mathbf{A}, \mathbf{G})$, OCT4 $(\mathbf{B}, \mathbf{H})$, and SOX2 (C, I), as well as for the cytoplasmic located marker LIN28 (D, J). SSEA4 (E, K) and TRA-1-60 (F, L), both located in the cell membrane were also detectable. The cell nuclei were stained with DAPI. Scale bar: $100 \mu \mathrm{m}$. 
The transcription factors NANOG, OCT4, and SOX2 (Fig. 12A - C, G - I) are located in the nuclei of both the BrS- and Ctrl-cells. LIN28 is located in the cytoplasm (Fig. 12D, J), whereas SSEA4 (Fig. 12E, K) and TRA1-60 (Fig. 12F, L) are located on the membrane surface. No significant difference among the analyzed hiPSC clones was observed.

\subsubsection{Activation of $N A N O G$ and $O C T 4$ promoters}

The methylation status of different CpGs in the promoter regions of NANOG and OCT4 was evaluated using sodium bisulfite sequencing assays, to confirm the endogenous activation of these pluripotency related genes in the generated hiPSCs compared to their parental hMSCs (Fig. 13). The promoter regions of OCT4 and NANOG were highly methylated in BM76 and BM77, whereas the $\mathrm{CpG}$ sites in hiPSCs of passage 8 to 16 showed a more unmethylated pattern. However, in all hiPSC clones the two promoter regions were still partially methylated compared to hESCs.

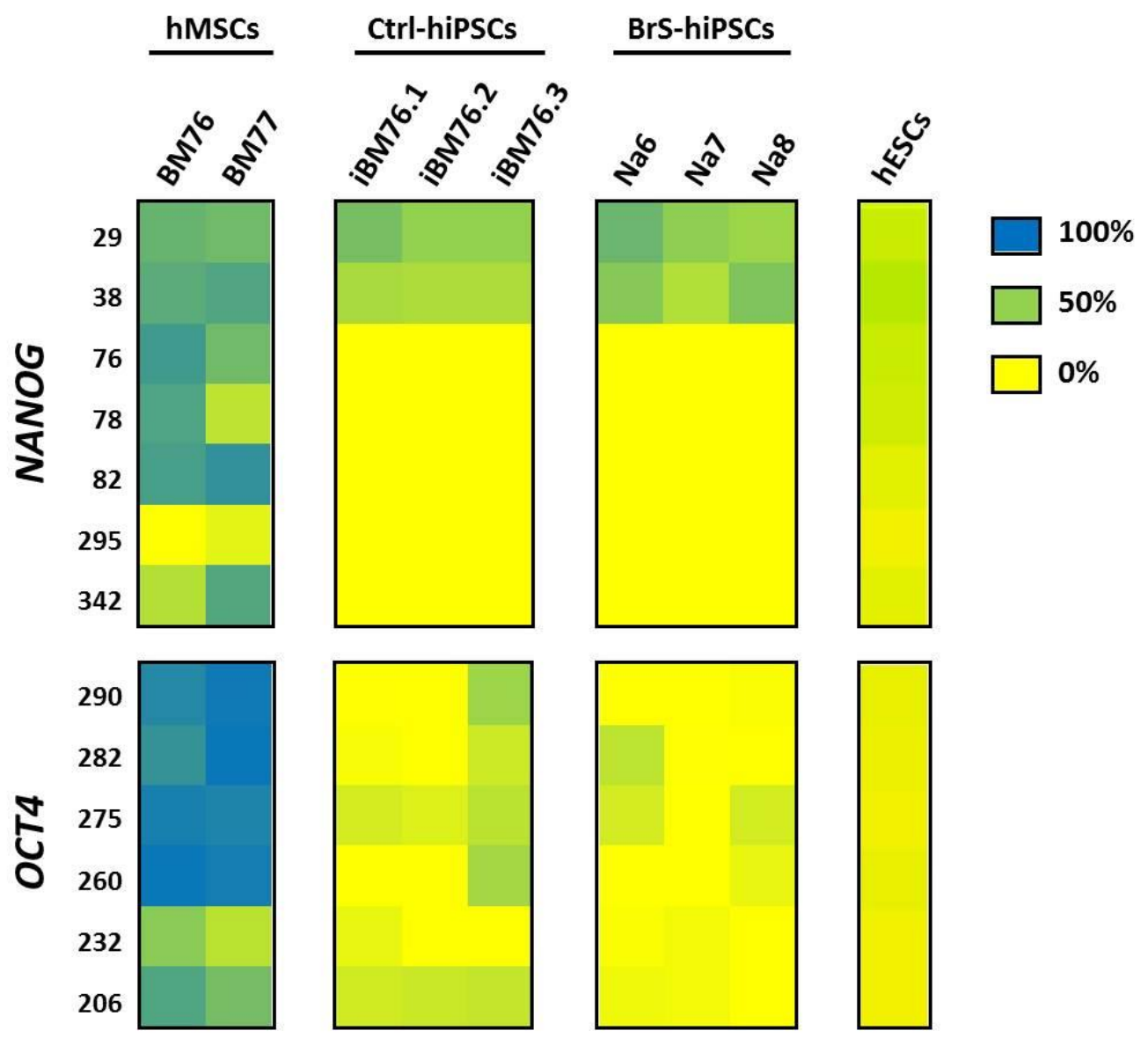

Figure 13. Methylation status of the promoter regions of pluripotency marker genes NANOG and OCT4. In comparison to their parental hMSCs (BM76, BM77), both promoter regions were more demethylated in all generated hiPSC clones. Human ESCs were used as positive control, where the analyzed $\mathrm{CpG}$ sites of both promoters were almost unmethylated. 


\subsubsection{Differentiation potential in vitro and in vivo}

The differentiation ability of the generated hiPSCs in vitro and in vivo was determined by the spontaneous differentiation protocol via EB formation and teratoma formation, respectively.

For the in vitro differentiation, the Ctrl- and BrS-hiPSCs were cultivated in suspension for 8 days in Iscove's medium. During this cultivation, the cells formed multi-cellular aggregates known as EBs, which were plated onto gelatin coated culture dishes at day 8 of differentiation. The mRNA was isolated and reverse transcribed into cDNA at three different time points (day 0 , day 8 , and day $8+25$ ). In all differentiation experiments, the cells showed a mRNA expression specific for all three embryonic germ layers in a developmentally controlled manner (Fig. 14). The early endodermal marker gene alpha-1-fetoprotein (AFP) was expressed at a low level at day 0 of differentiation and upregulated at day 8 , whereas albumin $(A L B)$, a late marker for hepatocytes, was highly expressed at late stages during the differentiation process. The same pattern was observed with the mesodermal marker genes cardiac troponin $T$ (cTNT) and alpha myosin heavy chain $(\alpha-M H C)$, which showed a very low level or no expression at day 0 , but an upregulation at day 8 . Both ectodermal marker genes, synaptophysin $(S Y P)$ and tyrosine hydroxylase $(T H)$ were also expressed in each differentiated cell clone.

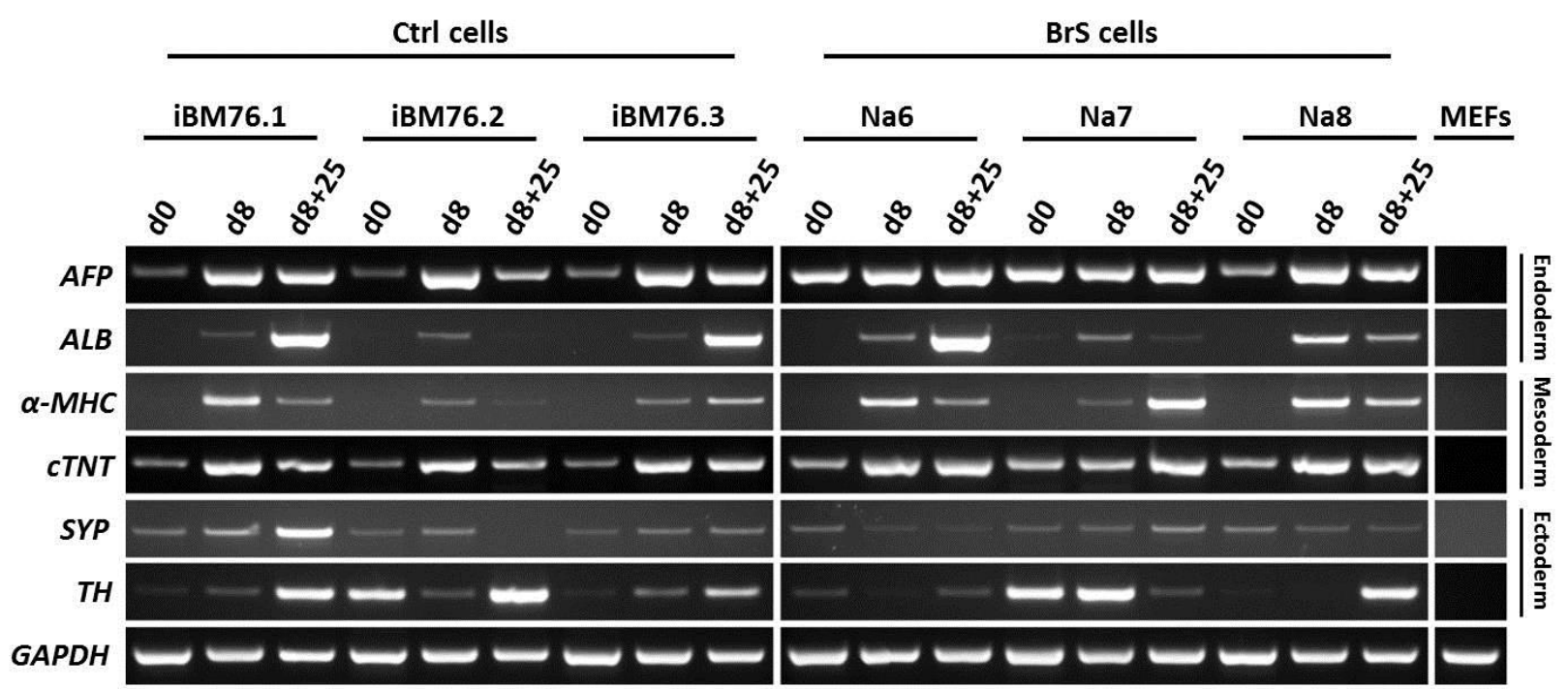

Figure 14. Gene expression analysis of differentiated hiPSCs. Ctrl- and BrS-hiPSCs were differentiated spontaneously in vitro and mRNA was isolated on day $0(\mathrm{~d} 0)$, day $8(\mathrm{~d} 8)$, and day $8+25(\mathrm{~d} 8+25)$ of differentiation. All analyzed genes are expressed in a developmentally controlled manner. MEFs were used as negative control. 
The differentiated hiPSCs were also stained with antibodies against three germ layer-specific proteins (Fig. 15). Both Ctrl- and BrS-hiPSCs were able to differentiate into cells, which were positive for the mesodermal marker smooth muscle actin (SMA, Fig. 15A, D). The neuroectodermal marker class III $\beta$-tubulin (Fig. 15B, E) as well as the endodermal marker AFP (Fig. 15C, F) were also detected among the differentiated cells.
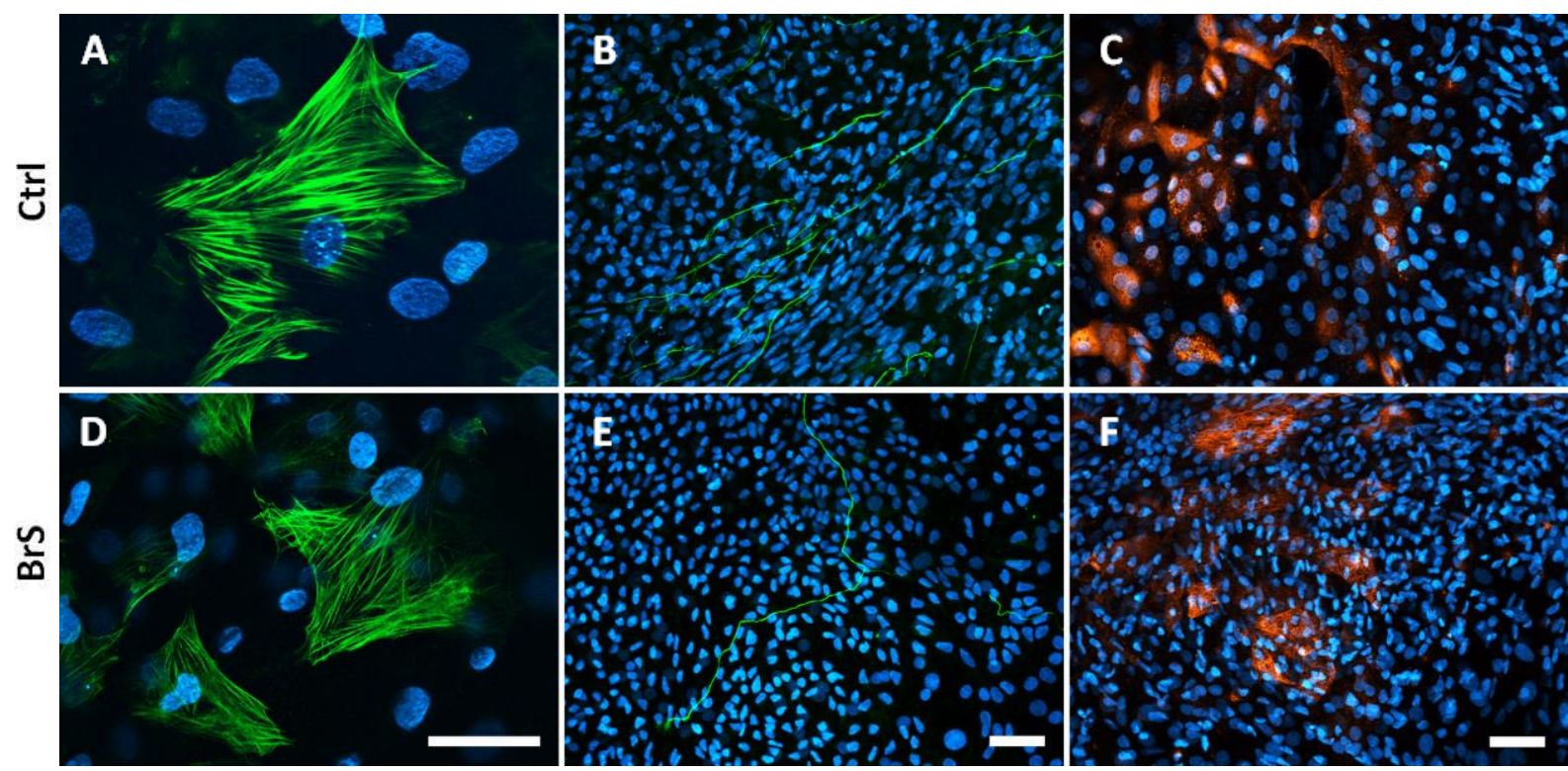

Figure 15. Immunostaining of differentiated hiPSCs detecting three germ layer-specific proteins. Both differentiated Ctrl- and BrS-hiPSCs were positive for the mesodermal marker protein SMA (A, D), the ectodermal marker class III $\beta$-tubulin $(\mathbf{B}, \mathbf{E})$ and the endodermal marker AFP $(\mathbf{C}, \mathbf{F})$. The cell nuclei were stained with DAPI. Scale bar: $50 \mu \mathrm{m}$.

The differentiation potential of the generated hiPSCs was also analyzed in vivo by subcutaneous injection of the cells into immunodeficient RAGC mice. The injected cells formed mature teratomas, which were analyzed histologically (Fig. 16). The teratomas derived from Ctrl-hiPSCs contained derivatives of all three embryonic germ layers, which showed cartilage (Fig. 16A) and muscle cells (Fig. 16B) as mesodermal tissue, neural rosettes (Fig. 16C) and intestinal tissue (Fig. 16D) representing ectodermal and endodermal tissues, respectively. The teratomas derived from BrS-hiPSCs contained mesodermal tissues represented by cartilage, muscle, and fat cells (Fig. 16E - G) as well as endodermal tissue (Fig. 16H). Ectodermal tissue was not found morphologically within the teratomas. 


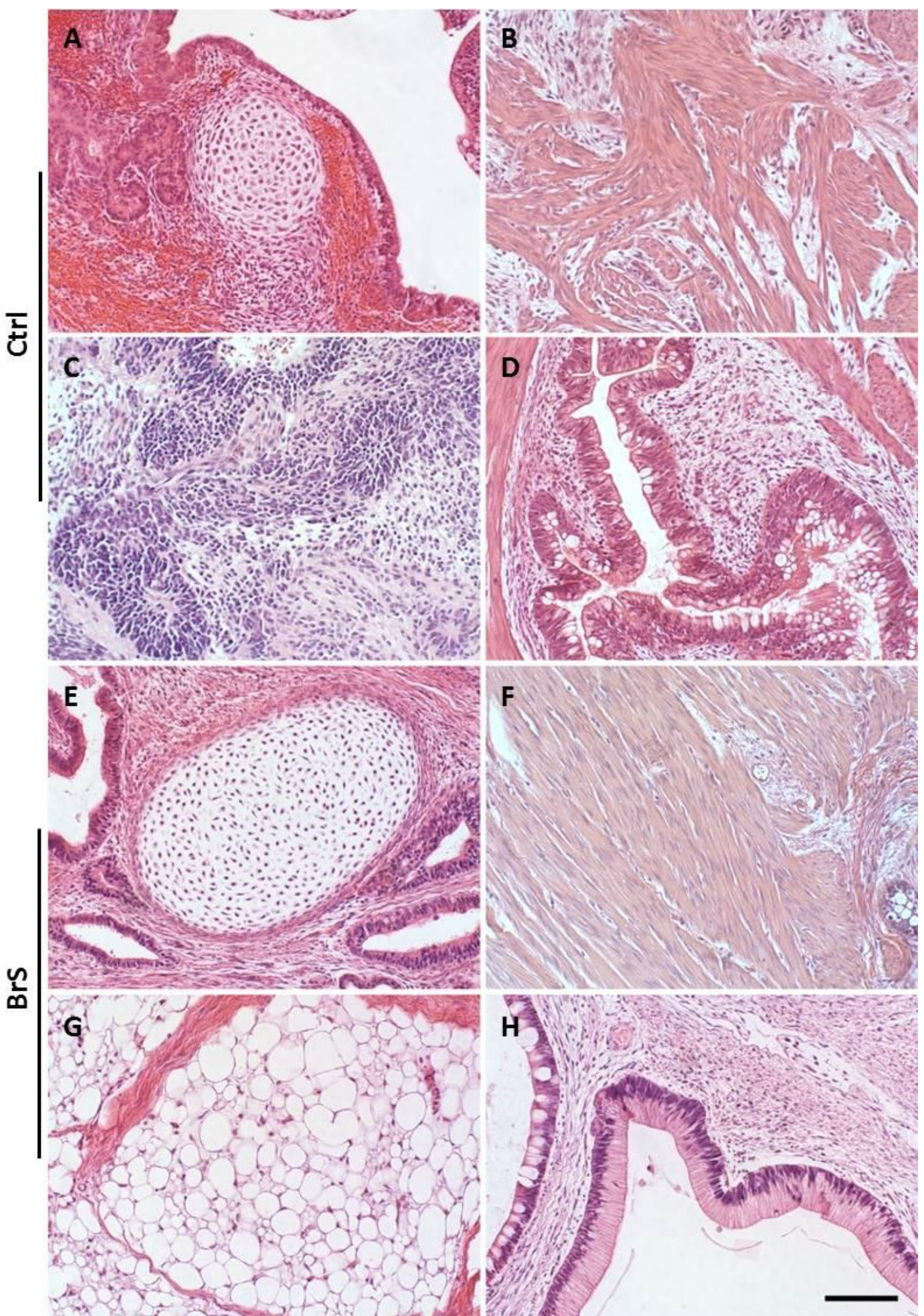

Figure 16. Teratoma formation of hiPSCs after subcutaneous injection into immunodeficient RAGC mice. Teratomas developed from Ctrl-hiPSCs $(\mathbf{A}-\mathbf{D})$ contained derivatives of all three embryonic germ layers: mesoderm represented by cartilage (A) and muscle cells (B), ectodermal neural rosettes (C), and intestinal tissue with endodermal origin (D). Teratomas derived from BrS-hiPSCs $(\mathbf{E}-\mathbf{H})$ contained derivatives of mesoderm, represented by cartilage $(\mathbf{E})$, muscle $(\mathbf{F})$, and fat $(\mathbf{G})$ as well as endoderm, represented by intestinal tissue $\mathbf{( H )}$. Scale bar: $100 \mu \mathrm{m}$. 
Taken together, the data show that the generated Ctrl- and BrS-hiPSCs are pluripotent. They express pluripotency related markers on mRNA and protein levels. The promoter regions of NANOG and OCT4 in the generated hiPSCs were demethylated, indicating that the endogenous expression of these two genes was activated. Furthermore, Ctrl- and BrS-hiPSCs were able to differentiate into derivatives of the three germ layers in vivo and in vitro.

\subsubsection{Karyotyping}

It is known that hESCs and hiPSCs might develop chromosomal instability under long-term culture conditions. The generated hiPSCs in this study were used for differentiation experiments until a maximum of passage 30. To determine whether these cells exhibit numerical aberrations of their chromosomes, hiPSCs in passage $\geq 30$ were analyzed. Seven out of $64(46, \mathrm{XX})$ in Ctrl-hiPSCs and 7 out of 83 BrS-hiPSCs (46, XY) exhibited an abnormal karyotype with 45 or less chromosomes. A trisomy of chromosome 12 or 17, which might provide a selective advantage for the propagation of undifferentiated cells, was not detected in the generated hiPSCs. Representative diploid karyograms for both Ctrl- and BrShiPSCs are shown in Figure 17.

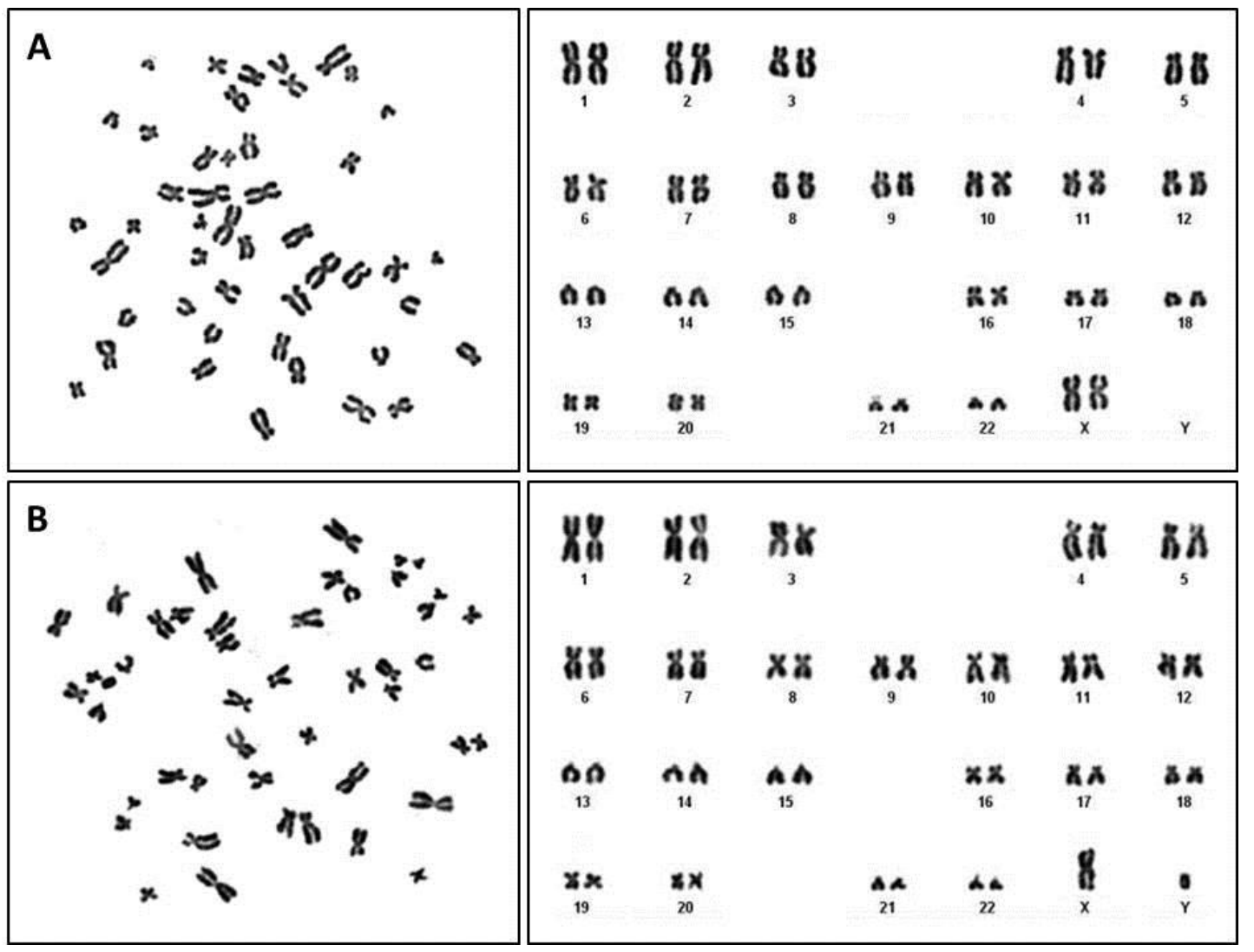

Figure 17. Karyotype of generated hiPSCs after long-term culture. Ctrl-hiPSCs (A) and BrS-hiPSCs (B) showed a normal karyotype of 46, XX and 46, XY, respectively. 
These data indicate that the majority of the cells have a normal karyotype.

\subsubsection{Verification of the SCN5A point mutation in BrS-hiPSCs}

The SCN5A gene region containing the mutant site found in the $\mathrm{BrS}$ patient was sequenced to confirm the genotypes in BrS- and Ctrl-hiPSCs. The genomic DNA of the generated hiPSCs was isolated and sequenced approximately $200 \mathrm{bp}$ up- and downstream of the predicted mutation at position 5435 within the SCN5A gene. As mentioned before, SCN5A is located on chromosome 3 p21 and codes for the $\alpha$-subunit of the cardiac sodium channel. All established BrS-hiPSCs showed the mutation C5435A referred to the coding sequence of the SCN5A transcript variant 1 (NCBI reference sequence: NM_198056.2; Fig. 18). All Ctrl-hiPSCs did not have this mutation and therefore both alleles carry a cytosine leading to the amino acid serine. In addition, the sequencing data revealed that all clones of both Ctrl- and BrS-hiPSCs exhibited a synonymous single nucleotide polymorphism (SNP) on position 5457 . Here, one allele carries a cytosine, whereas the other allele carries a thymine. On translational level both variants lead to the amino acid aspartic acid (p.D1819D).
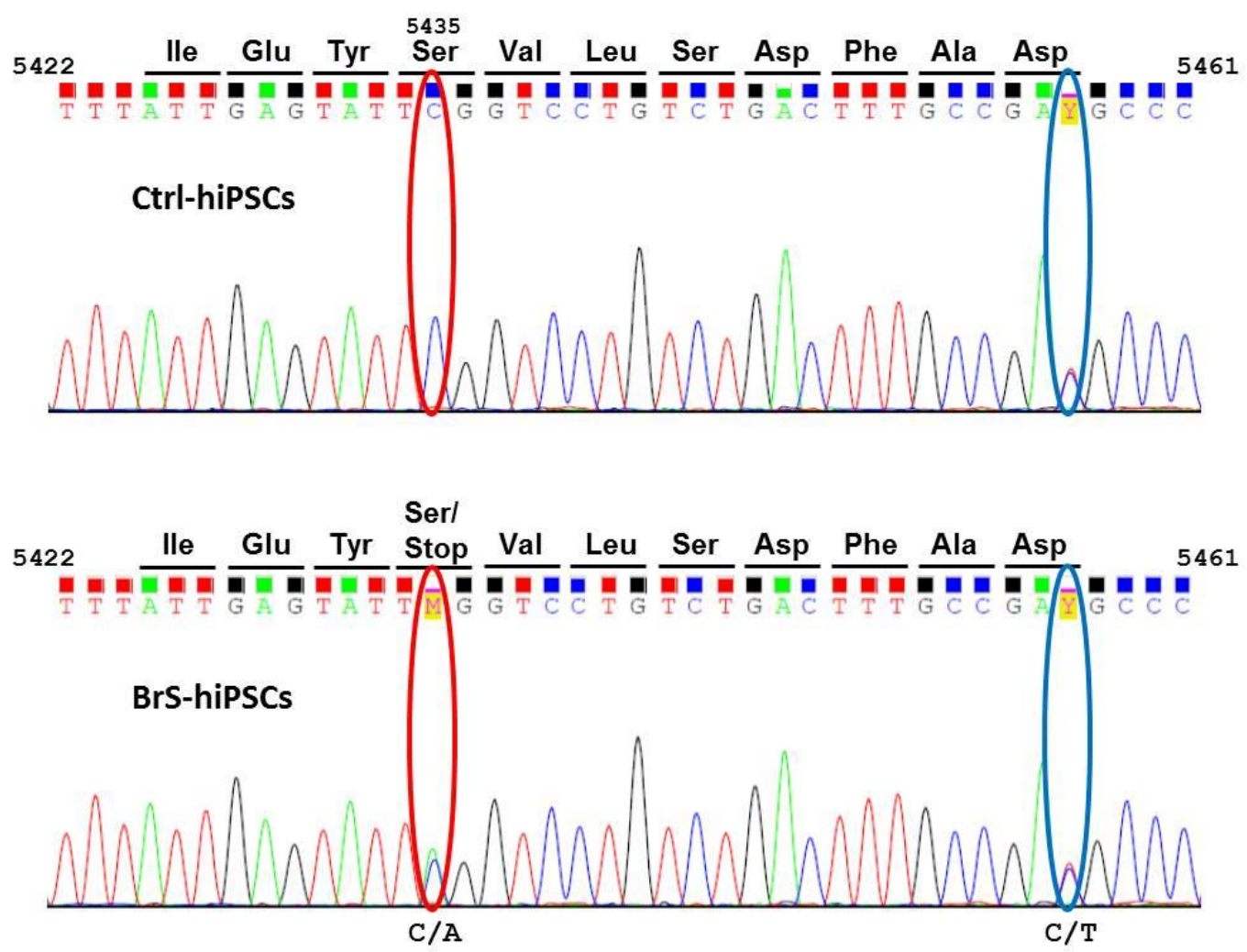

Figure 18. Verification of the $S C N 5 A$ point mutation in the generated BrS-hiPSCs. All BrScells exhibited the point mutation on position 5435 referred to the coding sequence of the $S C N 5 A$ transcript variant 1 , where a cytosine is replaced by an adenine on one of the alleles. The mutation leads to a premature stop codon. All Ctrl-cells did not show this mutation. The hiPSCs from both donors showed a synonymous SNP on position 5457, where one allele carries a cytosine and the other one carries a thymine. Both triplets code for aspartic acid (Asp). 


\subsection{Generation and phenotype characterization of hiPSC-derived cardiomyocytes}

\subsubsection{Ctrl- and BrS-hiPSCs differentiate into cardiomyocytes}

Ctrl- and BrS-hiPSCs as well as hESCs were differentiated into CMs using either the EB method (Fig. 19A) or direct differentiation method (Fig. 19B).

Approximately 2 - 3 days after plating the EBs onto gelatin coated dishes, first beating areas appeared. To assess the maturation of differentiated CMs, spontaneously beating areas at early stages (1 month) and late stages (3 months) were used for electrophysiological experiments. To avoid an overgrowth of noncardiac cell types in long-term culture, beating areas were manually picked and re-plated around day $8+25$ and allowed to further mature for an additional 2 months. About $8-42 \%$ of plated hiPSC-bodies and $16 \%$ of hESC-bodies contained beating clusters. No significant difference in differentiation efficiencies was observed between Ctrl- and BrS-hiPSCs. However, remarkable line-to-line differences and experiment-dependent variability resulted in inconsistent differentiation efficiencies. These factors were mainly associated with the cell quality (size, morphology and density of the colonies) before the initiation of the differentiation.

A

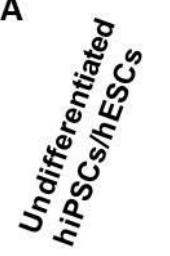

d0 d1
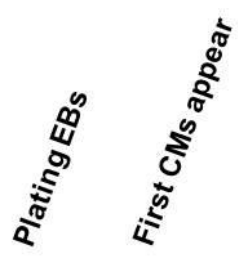

d8 $\mathrm{d} 8+3$

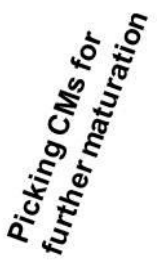

$\mathrm{d} 8+25$

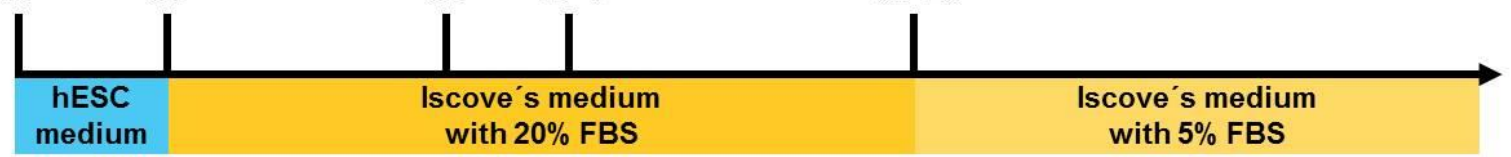

B
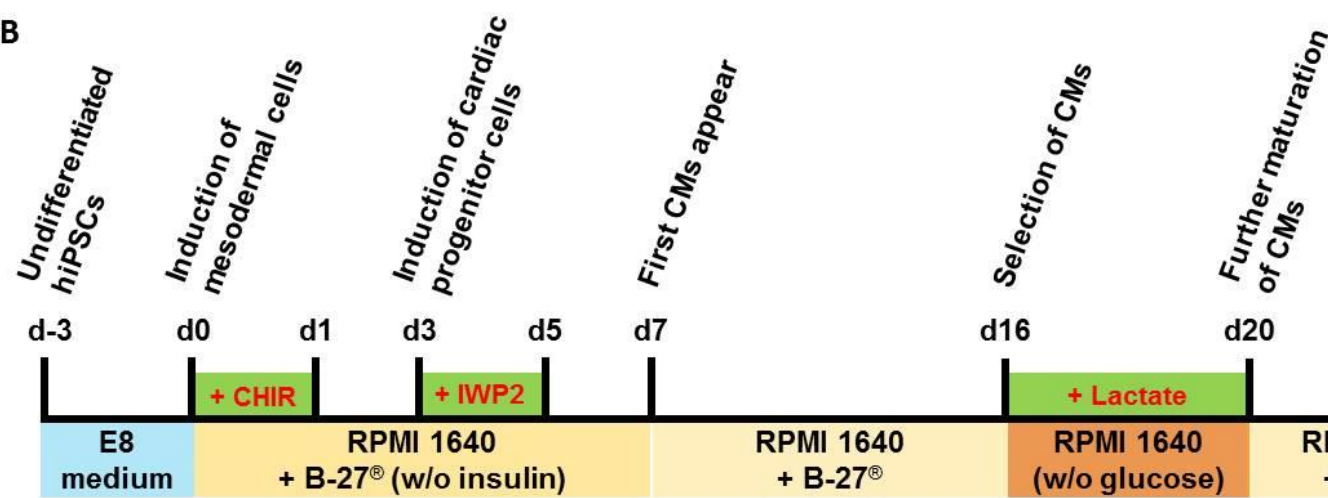

d7
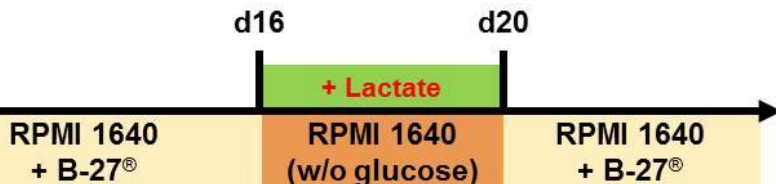

Figure 19. Schematic cardiac differentiation procedures of pluripotent stem cells using the EB method (A) and the directed differentiation method (B). 
In the directed cardiac differentiation experiments, first beating cells appeared at day $7-8$ of differentiation. Compared to the EB method, the directed differentiation method gave rise to much higher differentiation efficiencies of more than $50 \%$ by visual observation. Removal of glucose together with application of lactate in the culture medium for 4 days resulted in the purification of CMs higher than $90 \%$ by visual observation. No significant difference in differentiation efficiency was observed between Ctrl- and BrS-hiPSCs using the directed differentiation method.

CMs isolated from the beating clusters showed round, spindle-shaped, tri-, or multiangular morphologies. They displayed organized cross-striations following staining with antibodies against the myofilament proteins $\alpha$-actinin, myosin light chain 2a (MLC2a), and cTNT (Fig. 20). No significant difference was observed between Ctrl- and BrS-CMs regarding cardiac-specific structural proteins across all the cell clones. Additionally, the gap junction protein connexin 43 (Cx43) was detected between adjacent CMs, indicating a cell-to-cell coupling (Fig. 20A, D in green). Cx43 is important for conducting electrical signals between neighboring cells.
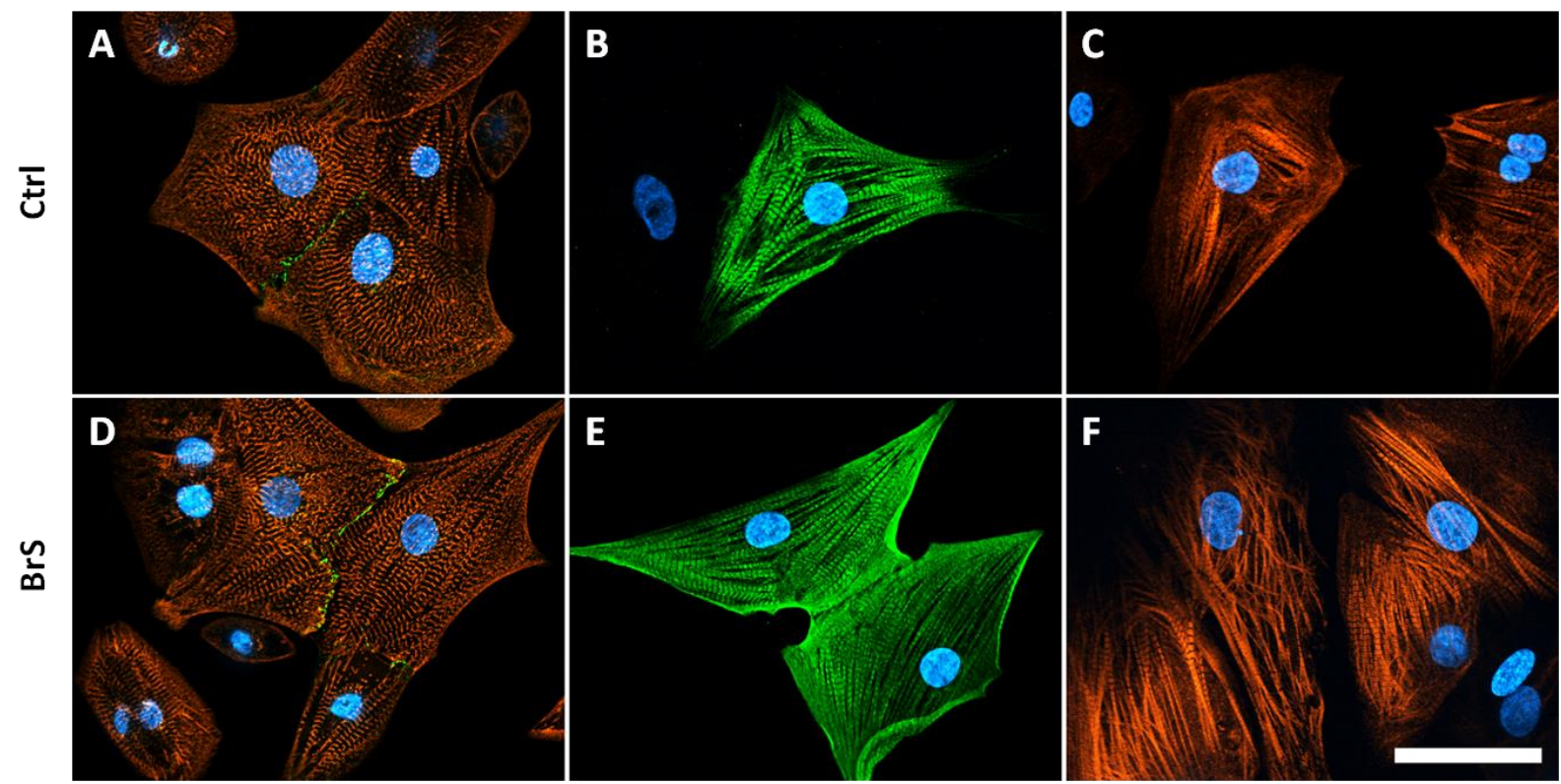

Figure 20. Cardiac differentiation of Ctrl- and BrS-hiPSCs. Isolated CMs expressed structural myofilament proteins $\alpha$-actinin $(\mathbf{A}, \mathbf{D}$ in red), cTNT $(\mathbf{B}, \mathbf{E})$, and MLC2a $(\mathbf{C}, \mathbf{F})$. The gap junction protein $\mathrm{Cx} 43$ was detected at the connection between adjacent CMs (A, D in green). Cell nuclei were stained with DAPI (in blue). Scale bar: $50 \mu \mathrm{m}$.

Furthermore, the generated hESC- and hiPSC-CMs responded to the exposure of known drugs. The beating frequency was measured before and after the addition of different concentrations of (S)-(-)-Bay K8644 and Diltiazem hydrochloride. Diltiazem blocks L-type 
calcium channels and hence leads to slower contraction rates of the CMs. Both hESC- and hiPSC-CMs stopped beating when exposed to Diltiazem hydrochloride with a concentration of $10^{-2} \mathrm{mM}$ (Fig. 21). In contrast, when the CMs were treated with the calcium channel agonist (S)-(-)-Bay K8644 the beating frequency raised significantly (Fig. 21). In hESC-CMs the beating frequency was raised up to $250 \%$ of the initial frequency at a concentration of $10^{-2} \mathrm{mM}$, whereas hiPSC-CMs could almost double their frequency.
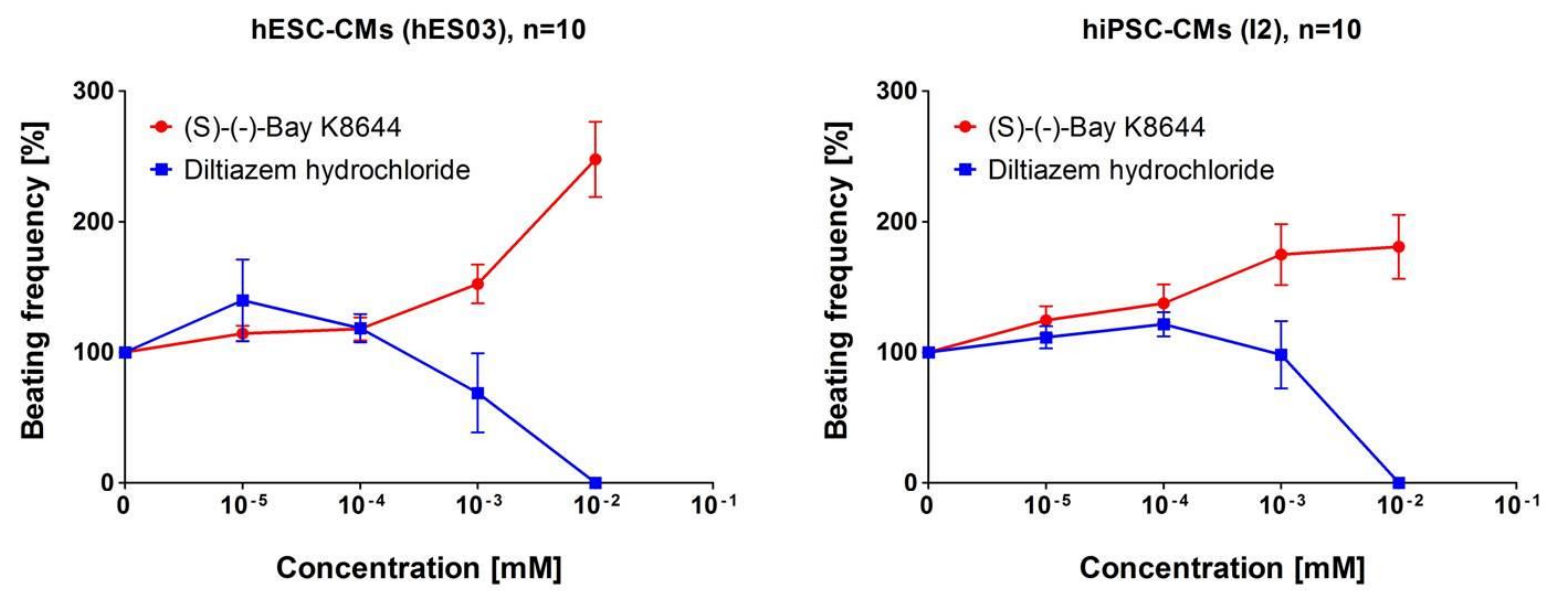

Figure 21. Response of generated hESC- and hiPSC-CMs when exposed to known drugs in different concentrations. Both, hESC- and hiPSC-CMs slightly increased their beating frequency when exposed to very low concentration of Diltiazem hydrochloride but decreased their frequency at higher concentrations until they stopped beating at $10^{-2} \mathrm{mM}$. When treated with (S)-(-)-Bay K8644 beating frequencies increased up to $250 \%$ of the initial frequency in hESC-CMs and $200 \%$ in hiPSC-CMs.

These data indicate that both Ctrl- and BrS-hiPSCs are able to differentiate efficiently into CMs displaying normal sarcomeric structures and the ability to respond to known drugs similar to hESC-CMs.

\subsubsection{BrS-CMs display reduced $I_{N a}$}

$I_{N a}$ density and gating properties of sodium channels were analyzed in single CMs to assess whether BrS-CMs recapitulate the disease phenotype in vitro.

It is known that voltage-gated sodium channels are activated by voltage changes across the cell membrane. The $I_{N a}$ was measured in 1- and 3-month-old hiPSC-CMs using the rupturedpatch whole-cell voltage clamp technique. The current-voltage (I-V) relationship was determined by changing the voltage from -95 to $+35 \mathrm{mV}$ in $5 \mathrm{mV}$ increments from a holding potential of $-100 \mathrm{mV}$. The I-V patch protocol and representative traces of I-V measurements in a Ctrl- and a BrS-CM are shown in Figure 22A. 
A
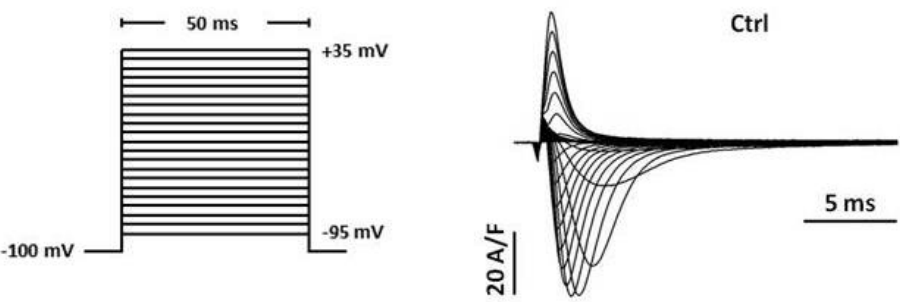

$\mathrm{BrS}$
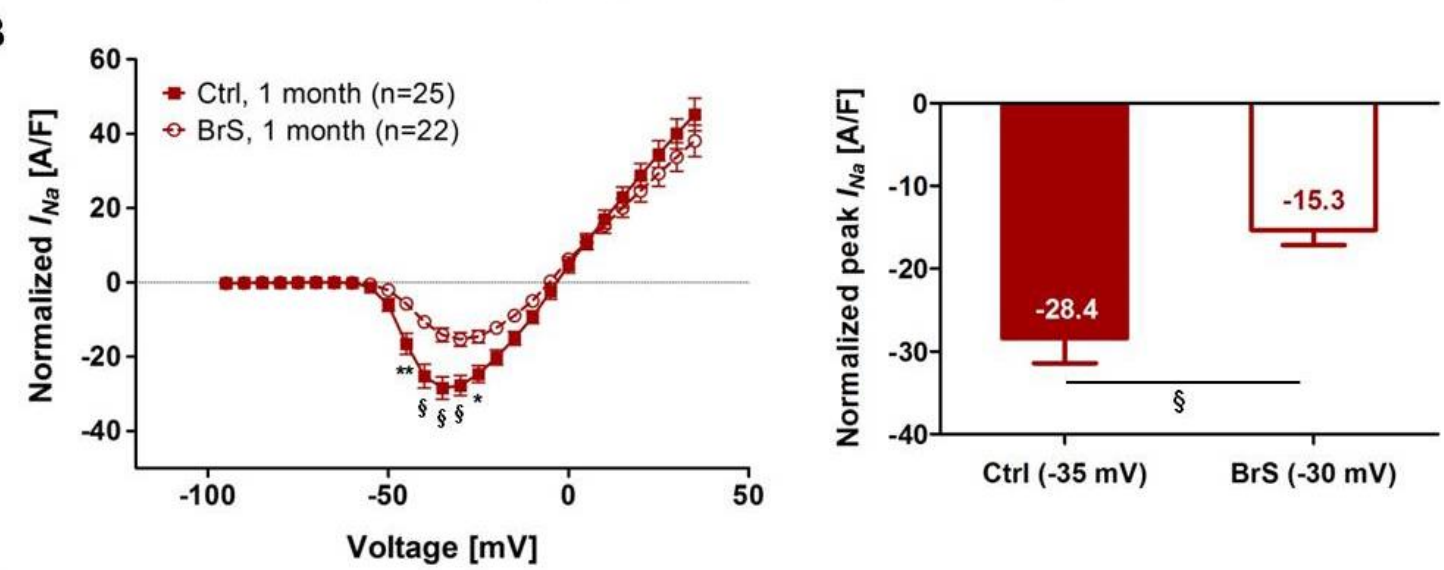

C
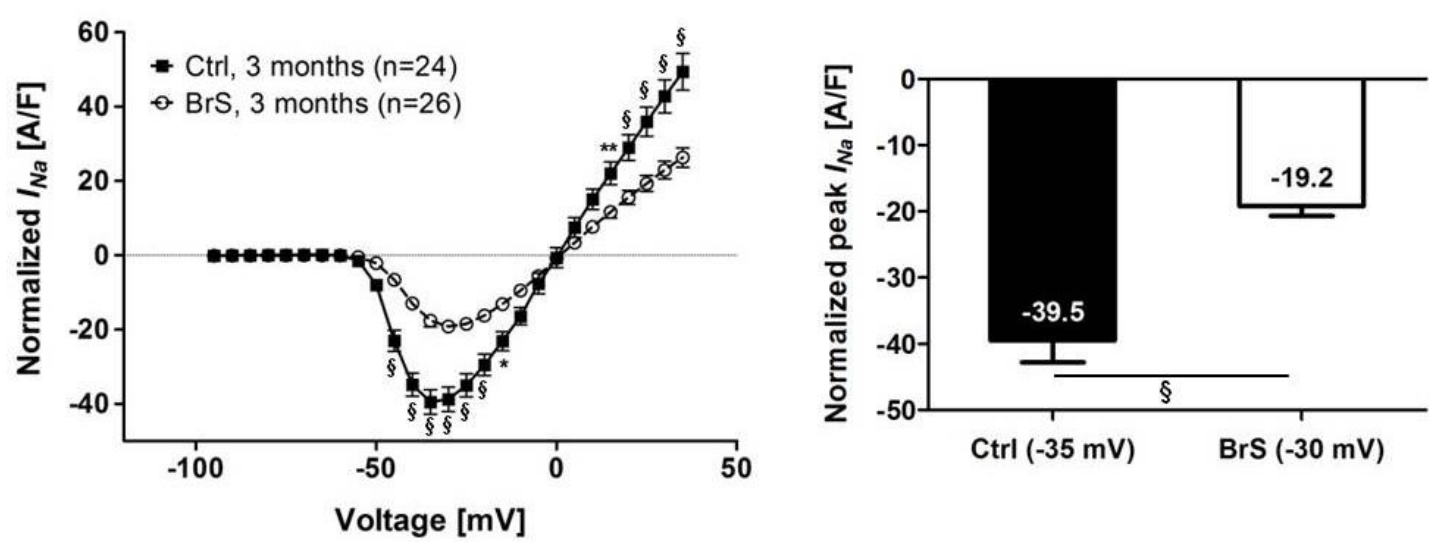

D
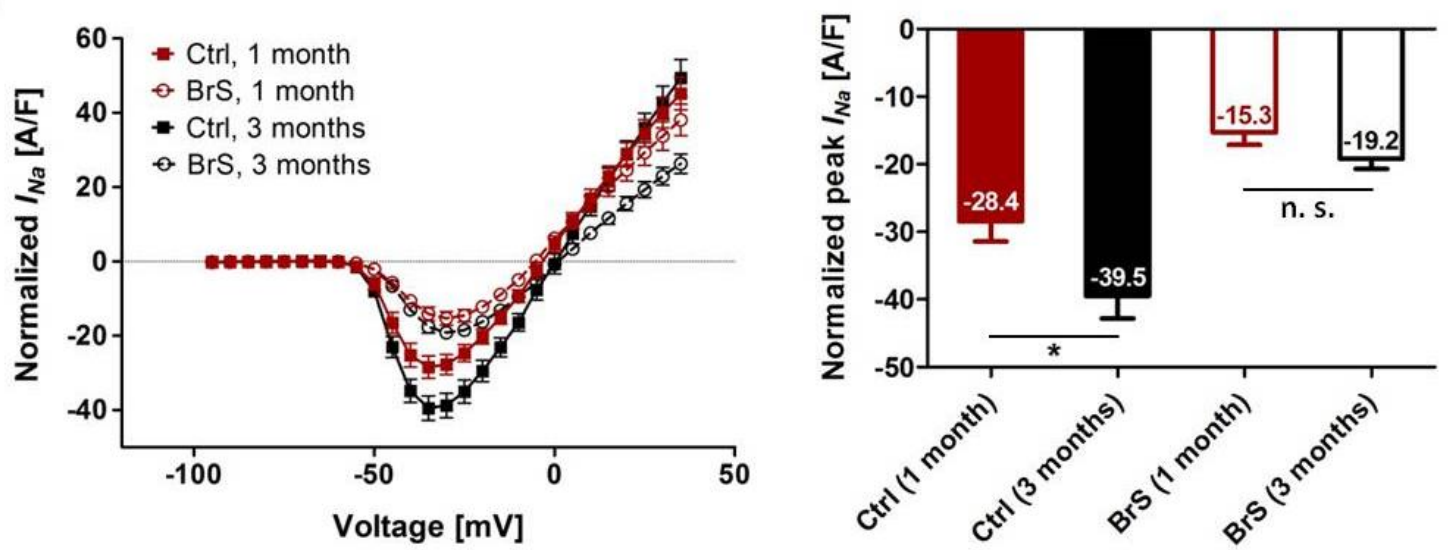

Figure 22. Sodium current-voltage (I-V) relationship in 1- and 3-month-old Ctrl- and BrS-CMs. The I-V protocol and representative $I_{N a}$ traces of a BrS- and a Ctrl-CM (normalized to $\mathrm{C}_{\mathrm{m}}$ ) are presented in $(\mathbf{A})$. $\mathrm{BrS}$ CMs showed a significant reduced $I_{N a}$ in both 1-month-old (B) and 3-month-old (C) cells compared to the Ctrlcells. Average $I_{N a}$ density (left) and the averaged maximum peak current density (right) in Ctrl- and BrS-CMs are presented. A right-shift of I-V curves was detected in BrS-CMs. $I_{N a}$ densities were increased in 3-month-old CMs compared to 1-month-old cells (D). Data are presented as mean \pm SEM. Two-way repeated measures ANOVA for I-V curves or unpaired Student's $t$-test for peak $I_{N a}$ densities were used for statistical analyses: $(*) \mathrm{p}<0.05,(* *) \mathrm{p}<0.01,(\S) \mathrm{p}<0.001, \mathrm{n} . \mathrm{s} .=$ not significant. 
The membrane capacitance of both 1- and 3-month-old BrS-CMs was comparable to 1- and 3month-old Ctrl-CMs (Table 11), respectively, which excludes possible functional differences relating to the cell size. A significant reduction of the average $I_{N a}$ density was observed in both 1- and 3-month-old BrS-CMs compared to the Ctrl-CMs (Fig. 22B, C). The maximal $I_{N a}$ density reached only $54 \%$ in 1-month-old BrS-CMs and $49 \%$ in 3-month-old BrS-CMs compared to their respective Ctrl-CMs.

Table 11. Biophysical parameters of the sodium channel in Ctrl- and BrS-CMs

\begin{tabular}{|c|c|c|c|c|}
\hline Parameter & Ctrl (1 month) & BrS (1 month) & Ctrl (3 months) & BrS (3 months) \\
\hline $\mathrm{C}_{\mathrm{m}}[\mathrm{pF}]$ & $48.90 \pm 3.63$ & $59.87 \pm 6.89$ & $58.22 \pm 5.23$ & $67.72 \pm 5.55$ \\
\hline $\mathrm{n}$ & 25 & 22 & 24 & 26 \\
\hline \multicolumn{5}{|c|}{ Steady-state activation } \\
\hline $\mathrm{V}_{1 / 2}[\mathrm{mV}]$ & $-40.99 \pm 0.27$ & $-36.95 \pm 0.19^{\S}$ & $-41,31 \pm 0.26$ & $-35.54 \pm 0.34^{\S}$ \\
\hline$\kappa[\mathrm{mV}]$ & $4.95 \pm 0.24$ & $5.35 \pm 0.16$ & $5.07 \pm 0.23$ & $5.90 \pm 0.29^{*}$ \\
\hline $\mathrm{n}$ & 25 & 22 & 24 & 26 \\
\hline \multicolumn{5}{|c|}{ Steady-state inactivation } \\
\hline $\mathrm{V}_{1 / 2}[\mathrm{mV}]$ & $-78.10 \pm 0.19$ & $-78.60 \pm 0.24$ & $-77.89 \pm 0.19$ & $-78.08 \pm 0.13$ \\
\hline$\kappa[\mathrm{mV}]$ & $5.47 \pm 0.17$ & $5.60 \pm 0,22$ & $5.75 \pm 0.17$ & $5.49 \pm 0.12$ \\
\hline $\mathrm{n}$ & 23 & 20 & 21 & 21 \\
\hline \multicolumn{5}{|c|}{ Intermediate inactivation } \\
\hline A & $0.091 \pm 0.006$ & $0.118 \pm 0.008$ & $0.095 \pm 0.006$ & $0.111 \pm 0.004$ \\
\hline$\tau[\mathrm{ms}]$ & $189.5 \pm 38.42$ & $156.8 \pm 29.85$ & $180.6 \pm 30.62$ & $165.3 \pm 15.14$ \\
\hline $\mathrm{n}$ & 22 & 20 & 19 & 21 \\
\hline \multicolumn{5}{|c|}{ Recovery from inactivation } \\
\hline A & $0.95 \pm 0.02$ & $0.88 \pm 0.02$ & $0.96 \pm 0.01$ & $0.91 \pm 0.01$ \\
\hline$\tau_{\mathrm{rec}}[\mathrm{ms}]$ & $11.71 \pm 0.56$ & $17.47 \pm 1.02^{\S}$ & $16.40 \pm 0.67$ & $14.94 \pm 0.59$ \\
\hline $\mathrm{n}$ & 23 & 22 & 22 & 24 \\
\hline
\end{tabular}

Membrane capacitance $\left(\mathrm{C}_{\mathrm{m}}\right)$, number of cardiomyocytes $(\mathrm{n})$, half-maximal voltage of steady-state (in)activation $\left(\mathrm{V}_{1 / 2}\right)$, slope factor of voltage dependence of (in)activation $(\kappa)$, amplitude of intermediate inactivation/recovery from inactivation $(\mathrm{A})$, time constant for development of intermediate inactivation $(\tau)$, time constant of recovery from inactivation $\left(\tau_{\text {rec }}\right)$. Data are presented as mean \pm SEM. Statistical analysis was performed between BrS- and Ctrl-CMs of the same developmental stage. Statistical significance is represented by $(*) \mathrm{p}<0.05$ and $(\S) \mathrm{p}<0.001$.

In addition, an increase of peak $I_{N a}$ densities was observed in prolonged cultures (Fig. 22D). Three-month-old Ctrl-CMs showed a significant increase of $39 \%$ whereas BrS-CMs demonstrated $26 \%$ (not significant) more current compared to their 1-month-old counterparts. 
Based on these data, at least 2-month-old CMs were further analyzed for the underlying molecular mechanisms in section 3.4.

A significant right shift of the I-V curve to a more positive potential in $\mathrm{BrS}-\mathrm{CM}$ s compared to Ctrl-CMs was detected (Fig. 22B, C). The maximal current in Ctrl-CMs appeared at the membrane potential of $-35 \mathrm{mV}$, whereas in $\mathrm{BrS}-\mathrm{CMs}$ it appeared at $-30 \mathrm{mV}$. This right shift is reflected in the activation curves of the sodium channels (Fig. 23B, C). The potential of half-maximal steady-state activation $\left(\mathrm{V}_{1 / 2}\right)$ of sodium channels in 1- and 3-month-old CtrlCMs was $-40.99 \mathrm{mV}$ and $-41.31 \mathrm{mV}$ with a slope factor $\kappa$ of $4.95 \mathrm{mV}$ and $5.07 \mathrm{mV}$, respectively (Table 11, Fig. 23B, C). $\mathrm{V}_{1 / 2}$ of steady-state activation shifted significantly towards more positive potentials in BrS-CMs (1-month-old: $\mathrm{V}_{1 / 2}=-36.95 \mathrm{mV}, \kappa=5.35 \mathrm{mV}$; 3-month-old: $\mathrm{V}_{1 / 2}=-35.54 \mathrm{mV}, \kappa=5.90 \mathrm{mV}$ ). However, $\mathrm{V}_{1 / 2}$ of steady-state activation remained unchanged in 3-month-old Ctrl- or BrS-CMs compared to their respective 1-monthold cells.

These data demonstrate that CMs derived from BrS-hiPSCs display a significant reduction of $I_{\mathrm{Na}}$ and a significant right shift of the I-V and activation curves. In addition, 3-month-old CMs exhibit larger $I_{N a}$ than 1-month-old CMs, indicating functional maturation of the cells.

\subsubsection{Sodium channels of BrS-CMs show no significant changes in steady-state inactivation, intermediate inactivation, and recovery from inactivation}

The differentiated CMs were held at various membrane potentials from $-120 \mathrm{mV}$ to $-20 \mathrm{mV}$ for $500 \mathrm{~ms}$ in $5 \mathrm{mV}$ increments to determine the voltage-dependent steady-state inactivation (availability) of sodium channels. A short (20 ms) test pulse of $-20 \mathrm{mV}$ was given after this steady-state to elicit remaining sodium currents (Fig. 23A, left, voltage protocol; right, example of $I_{N a}$ traces). In contrast to the activation curves, the steady-state inactivation of sodium channels did not differ between BrS- and Ctrl-CMs (Fig. 23B, C) as indicated in the half-maximal voltage of inactivation $\left(\mathrm{V}_{1 / 2}\right)$ and the slope factor $\kappa$ (Table 11). $\mathrm{V}_{1 / 2}$ in 1-monthold Ctrl-CMs was $-78.10 \mathrm{mV}$ with a slope factor $\kappa$ of $5.47 \mathrm{mV}$ compared to $\mathrm{V}_{1 / 2}=-78.60 \mathrm{mV}$ and $\kappa=5.60 \mathrm{mV}$ in BrS-CMs (Table 11). No significant differences in steady-state inactivation were detected between 1- and 3-month-old Ctrl-CMs and BrS-CMs, respectively. 
A

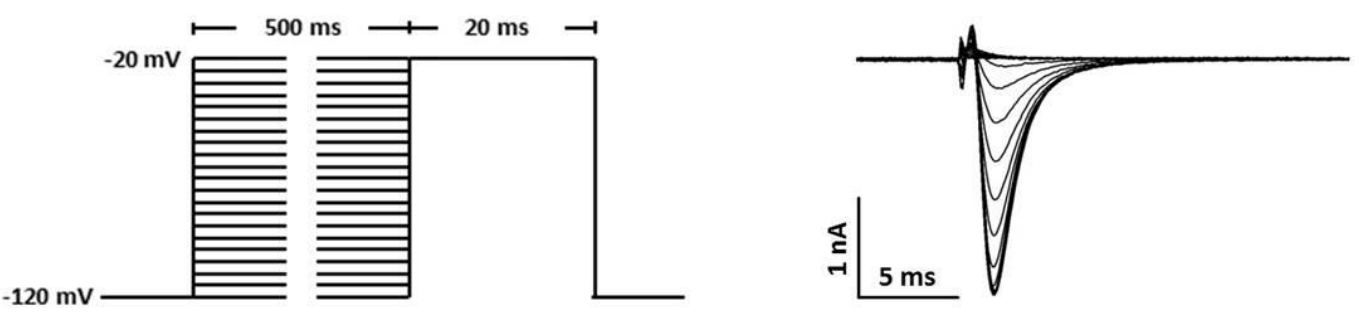

B

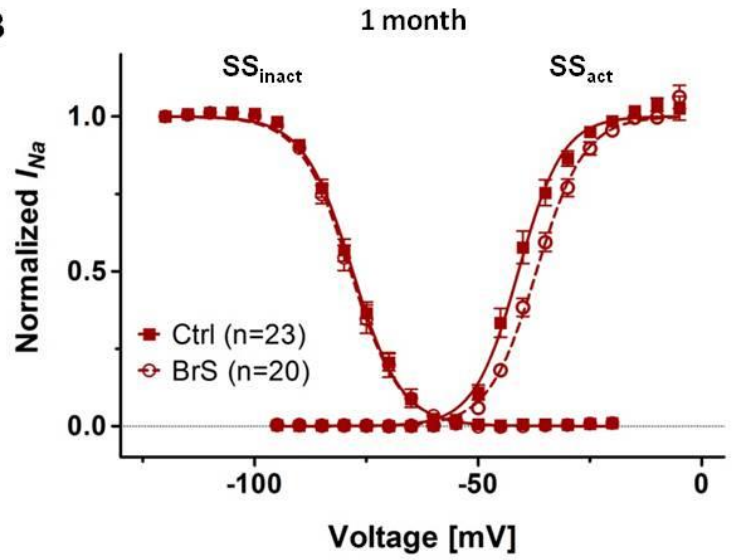

C

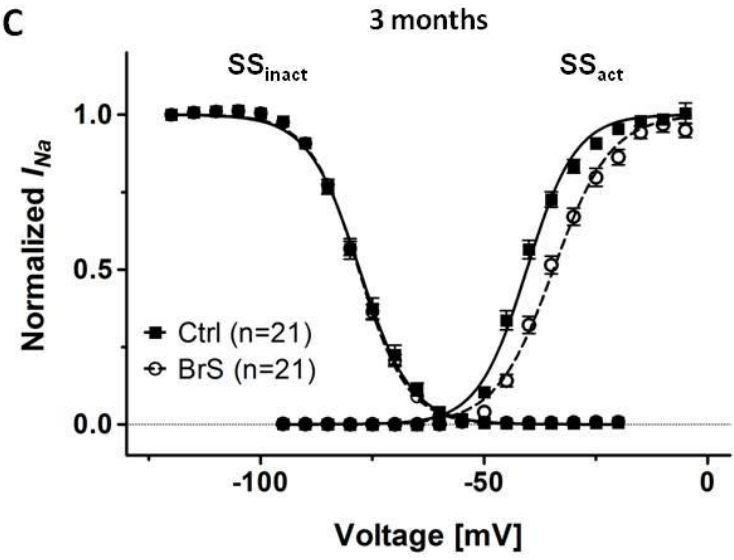

Figure 23. Steady-state activation ( $\left.\mathrm{SS}_{\text {act }}\right)$ and inactivation $\left(\mathrm{SS}_{\text {inact }}\right)$ of cardiac sodium channels in 1- and 3month-old Ctrl- and BrS-CMs. The inactivation protocol and a typical inactivation trace are presented in (A). The average voltage dependence of inactivation was not affected in BrS-CMs compared to Ctrl-CMs in both, 1(B) and 3-month-old (C) cells. The voltage dependent activation in BrS-CMs is characterized by a right shift of their activation curves compared to Ctrl-CMs, indicating a delayed activation of the sodium channels in BrSCMs. Data are presented as mean \pm SEM and summarized in Table 11 .

To study the intermediate inactivation of the sodium channels, a double pulse protocol with depolarization pulses from -100 to $-20 \mathrm{mV}$ was applied. The time length of the first pulse was increased from 15 to $1054 \mathrm{~ms}$ within a series of measurements (Fig. 24A). The timedependent availability of sodium channels was monitored at the second pulse. The amplitude of intermediate inactivation (A), representing the fraction of sodium channels that enter the inactivated state, was not significantly changed between Ctrl- and BrS-CMs in both 1- and 3month-old cells (Fig.24B, C; Table 11). The time constants for development of intermediate inactivation $(\tau)$ were slightly increased in Ctrl-CMs compared to BrS-CMs, showing that sodium channels in BrS-CMs inactivate not significantly faster compared to Ctrl-CMs. In addition, the amplitude of intermediate inactivation and the time constants for development of intermediate inactivation did not exhibit significant changes with the maturation status of the cells (1- vs. 3-month-old cells in both Ctrl- and BrS-cells; Fig. 24D). 
A
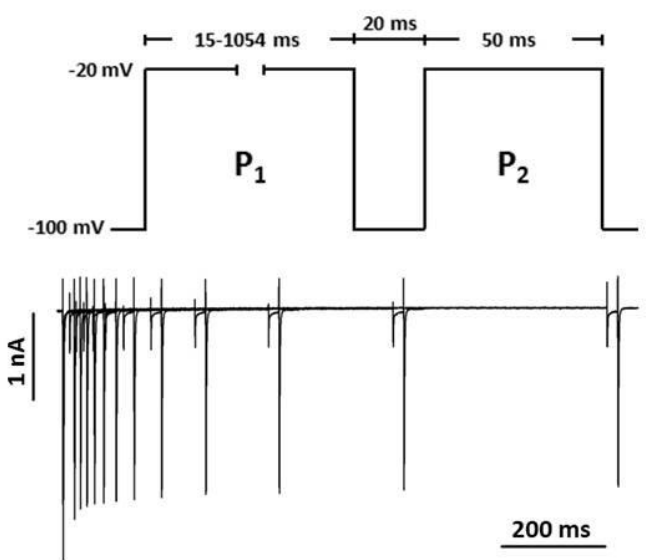

C

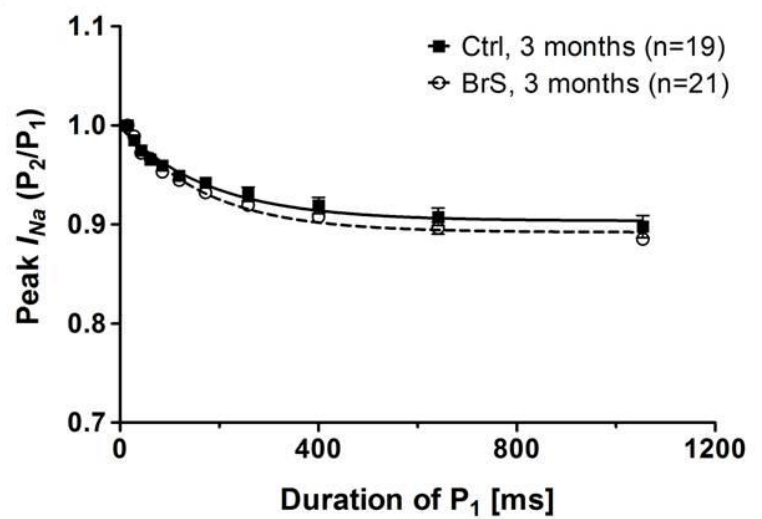

B

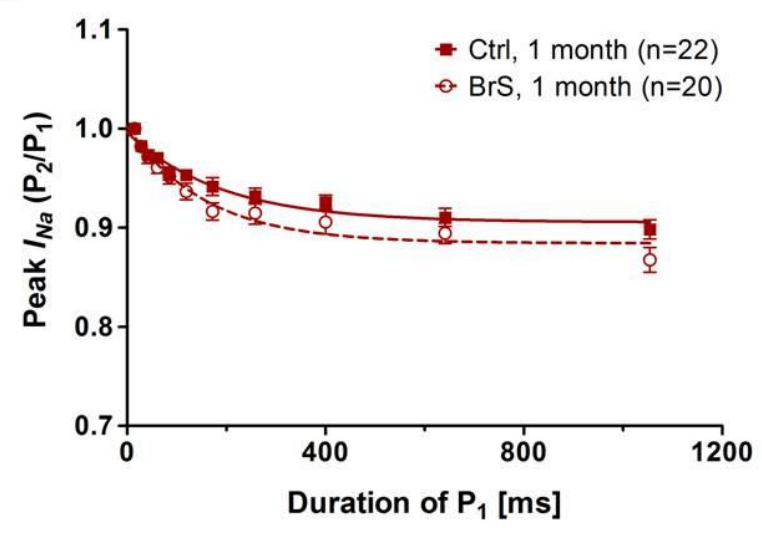

D

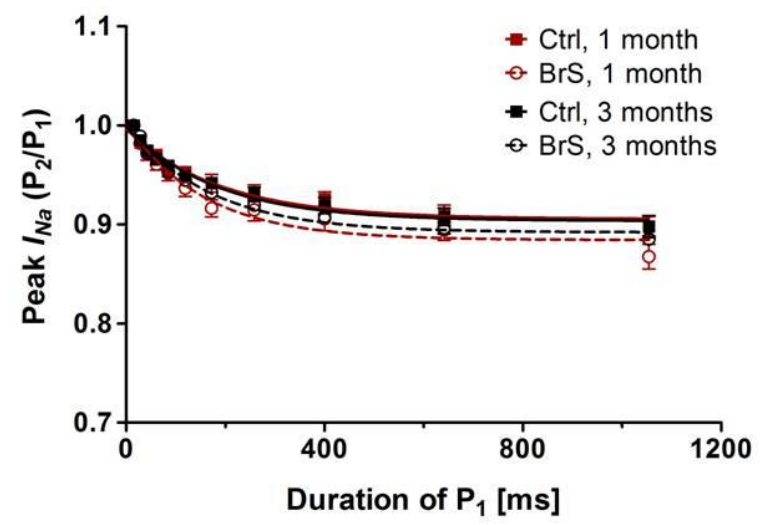

Figure 24. Intermediate inactivation of cardiac sodium channels in 1- and 3-month-old Ctrl- and BrSCMs. The inactivation protocol and a typical trace of intermediate inactivation are presented in (A). The development of intermediate inactivation was not significantly affected in BrS-CMs compared to Ctrl-CMs in both 1- (B) and 3-month-old (C) cells. The inactivation did also not change with the maturation status of the cells (D). Data are presented as mean \pm SEM and summarized in Table 11.

The rate of sodium channel recovery of inactivation was next investigated by using a double pulse protocol with an increasing delay from $1-165 \mathrm{~ms}$ between the first and the second pulse (Fig. 25A). CMs were depolarized from -100 to $-20 \mathrm{mV}$ for $1000 \mathrm{~ms}$ to initiate sodium channel inactivation. A longer delay period between the two pulses resulted in the recovery of a higher fraction of sodium channels at the second pulse of $-20 \mathrm{mV}$ (Fig. 25A). The recovery from inactivation was significantly faster in 1-month-old Ctrl-CMs compared to BrS-CMs (Fig. 25B; Table 11), whereas no significant differences were observed in 3-month-old CtrlCMs compared to BrS-CMs (Fig. 25C; Table 11). Seemingly, the rate of recovery in CtrlCMs was decreased upon maturation and there was no significant difference between $\mathrm{BrS}$ and Ctrl-CMs anymore at the developmental stage of 3 months (Fig. 25C, D). 
A

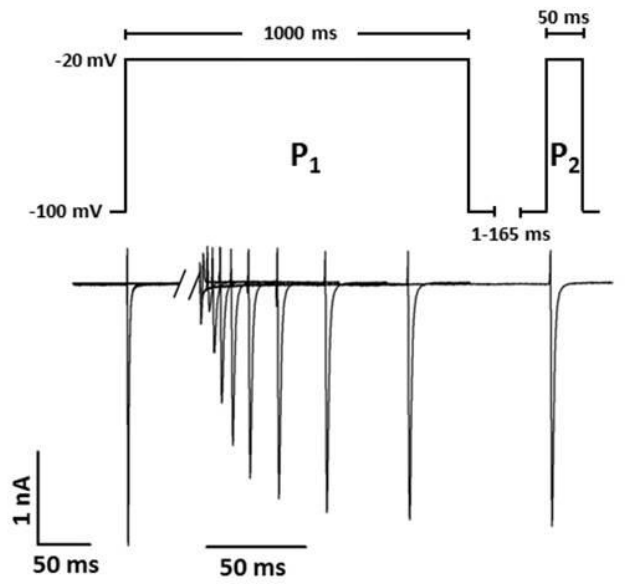

C

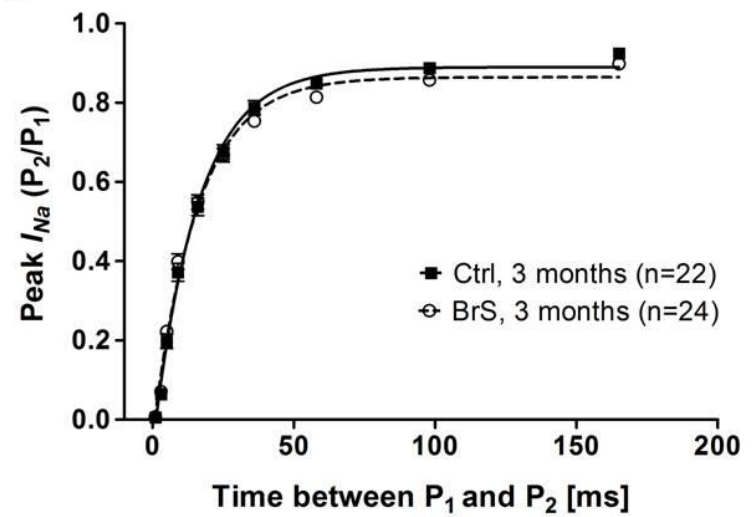

B

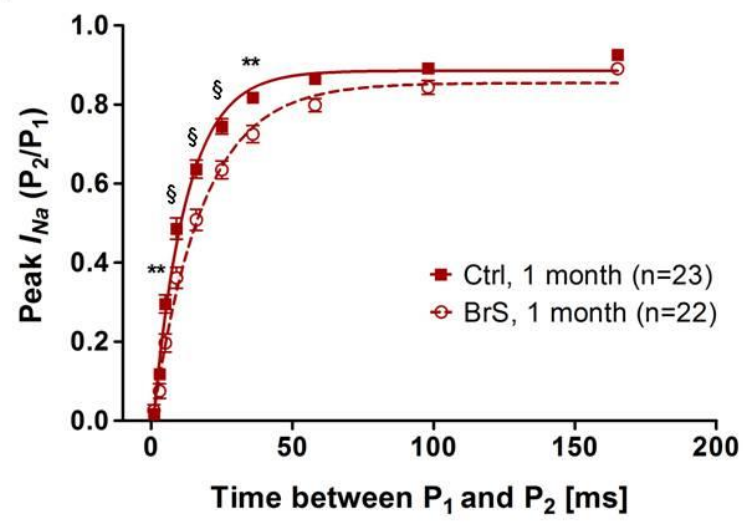

D

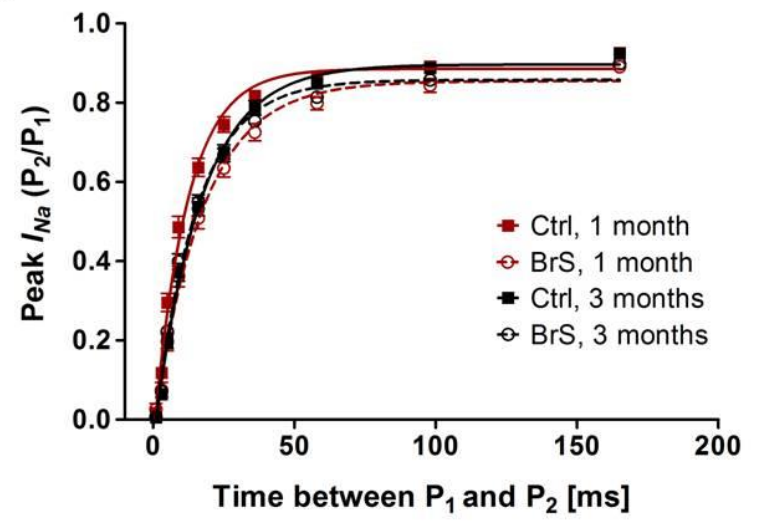

Figure 25. Recovery from inactivation of cardiac channels in 1- and 3-month-old Ctrl- and BrS-CMs. The protocol for recovery from inactivation and a typical trace are presented in (A). 1-month-old Ctrl-CMs recovered significantly faster than BrS-CMs (B). There was no significant difference in recovery between 3month-old Ctrl- and BrS-CMs (C), which may be due to a slower recovery in 3-month-old Ctrl-CMs (D). Data are presented as mean \pm SEM and summarized in Table 11. Two-way repeated measures ANOVA was used for statistical analysis. Statistical significance is represented by $(* *) p<0.01$ and $(\S) p<0.001$.

Taken together, these data show that the steady-state inactivation and the recovery from inactivation of sodium channels in 3-month-old BrS-CMs are comparable to Ctrl-CMs. However, the intermediate inactivation of cardiac sodium channels is slightly, but not significantly enhanced in BrS-CMs.

\subsubsection{BrS-CMs show no significant changes in persistent $I_{N a}$}

Persistent $I_{N a}$ in cardiac cells may have a substantial contribution to $\mathrm{Na}^{+}$ion loading during each cardiac cycle and contribute to triggered arrhythmia by causing repolarization failure (EADs), or by inducing delayed afterdepolarizations (DADs) attributable to calcium oscillations in $\mathrm{Na}^{+} / \mathrm{Ca}^{2+}$ ion overload conditions. To study whether the SCN5A mutation C5435A (p.S1812X) leads to an enhanced $I_{N a}$ in BrS-CMs, persistent $I_{N a}$ density was 
measured in the interval between $50-450 \mathrm{~ms}$ of a $1000 \mathrm{~ms}$ pulse in 3-month-old CMs (Fig. 26A). No significant difference between BrS- and Ctrl-CMs could be detected (Fig 26B).

A

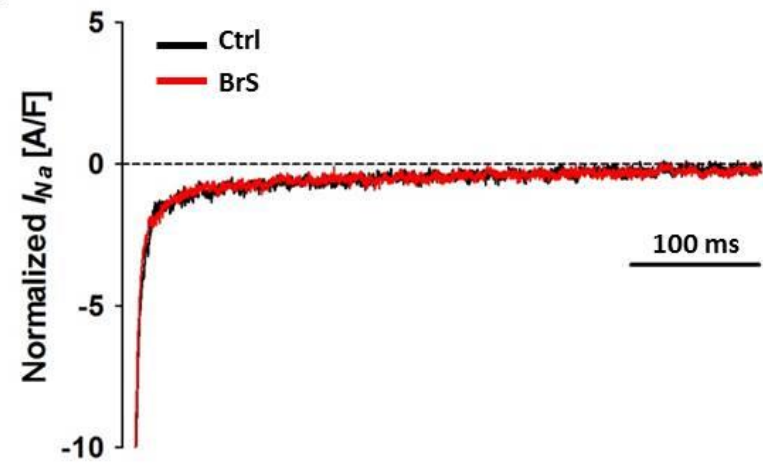

B

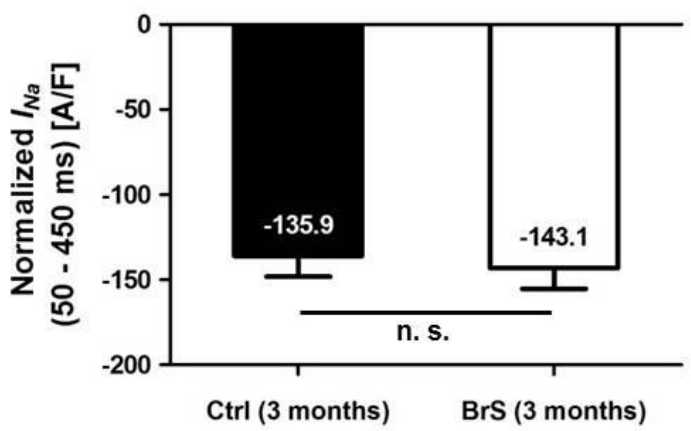

Figure 26. Persistent $\boldsymbol{I}_{\mathrm{Na}}$ in 3-month-old CMs. Normalized $I_{\mathrm{Na}}$ recordings of a representative Ctrl- (black) and BrS-CM (red) are presented as an overlay (A). The normalized persistent $I_{N a}$ density in the time interval between $50-450 \mathrm{~ms}(\mathbf{B})$ showed no significant difference between BrS- $(\mathrm{n}=24)$ and Ctrl-CMs $(\mathrm{n}=19)$. Data are presented as mean \pm SEM. Student's unpaired $t$-test was used for statistical analysis (n. s. $=$ not significant).

\subsubsection{Action potentials of BrS-CMs exhibit a reduced $\mathrm{V}_{\max }$}

To assess whether the BrS-CMs can recapitulate the disease phenotype in vitro, APs in single BrS-CMs compared to Ctrl-CMs were recorded. Electrophysiological studies showed that both Ctrl- and BrS-CMs generated APs spontaneously. Three major cardiac subtypes (embryonic ventricular-, atrial-, and nodal-like) were identified in Ctrl- and BrS-CMs based on AP morphologies. In addition to the AP shape, stringent criteria were defined to classify the cardiac subtypes for further analyses. The morphologies of different APs are shown in Fig. 27.
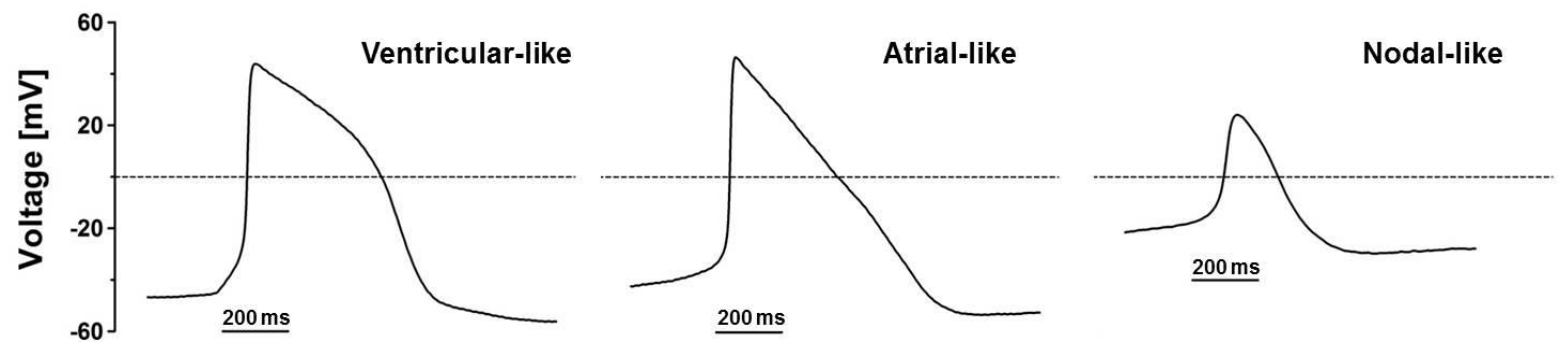

Figure 27. Classification of differentiated CMs according to their AP morphologies. The APs of a ventricular-like CM are characterized by a very negative RMP, a rapid AP upstroke, and a long plateau phase, whereas the APs of an atrial-like CM lack the plateau and instead show a more triangular-like shape. A typical AP of a nodal-like CM has a less negative RMP and a slower upstroke velocity. Furthermore, the AP amplitude is much shorter in these cells compared to ventricular- or atrial-like CMs. 
The typical AP of a ventricular-like $\mathrm{CM}$ is characterized by a relative negative RMP $(<-60 \mathrm{mV})$, a rapid AP upstroke, an APA bigger than $95 \mathrm{mV}$, and a prominent plateau phase. The atrial-like AP exhibits similar properties but lacks the plateau phase, leading to a triangular-like shape. A nodal-like AP exhibits typically a less negative RMP ( $\geq-55 \mathrm{mV})$, an APA smaller than $85 \mathrm{mV}$ and a very slow maximal upstroke velocity $\left(\mathrm{V}_{\max } \leq 5 \mathrm{~V} / \mathrm{s}\right)$.

The majority of the analyzed cells were found to be ventricular-like cells (Table 12). On average, the ventricular- and atrial-like CMs showed a slightly more negative RMP in BrSCMs compared to Ctrl-CMs, whereas the APA showed no difference. The characteristics of nodal-like APs were similar between Ctrl- and BrS-CMs (Table 12).

Table 12. Action potential characteristics

\begin{tabular}{|c|c|c|c|}
\hline & $\mathbf{R M P}[\mathbf{m V}]$ & APA $[\mathrm{mV}]$ & $\mathbf{V}_{\max }[\mathrm{V} / \mathrm{s}]$ \\
\hline \multicolumn{4}{|l|}{ Ctrl (1 month) } \\
\hline Ventricular-like $(\mathrm{n}=9)$ & $-69.7 \pm 1.2$ & $107.3 \pm 2.0$ & $17.9 \pm 2.2$ \\
\hline Atrial-like $(\mathrm{n}=3)$ & $-67.5 \pm 1.4$ & $102.2 \pm 2.6$ & $19.1 \pm 3.0$ \\
\hline Nodal-like $(\mathrm{n}=2)$ & $-54.6 \pm 3.6$ & $71.9 \pm 13.5$ & $2.5 \pm 0.7$ \\
\hline \multicolumn{4}{|l|}{ BrS (1 month) } \\
\hline Ventricular-like $(n=19)$ & $-73.1 \pm 1.2$ & $106.3 \pm 1.4$ & $17.8 \pm 1.8$ \\
\hline Atrial-like $(\mathrm{n}=3)$ & $-72.9 \pm 1.4$ & $108.5 \pm 5.2$ & $16.2 \pm 7.1$ \\
\hline Nodal-like $(\mathrm{n}=1)$ & -48.4 & 61.0 & 3.5 \\
\hline \multicolumn{4}{|l|}{ Ctrl (3 months) } \\
\hline Ventricular-like $(\mathrm{n}=17)$ & $-66.1 \pm 1.1$ & $105.8 \pm 1.4$ & $25.1 \pm 0.8$ \\
\hline Atrial-like $(\mathrm{n}=1)$ & -69.4 & 91.5 & 20.7 \\
\hline Nodal-like $(\mathrm{n}=3)$ & $-54.7 \pm 1.8$ & $80.3 \pm 2.7$ & $1.7 \pm 0.1$ \\
\hline \multicolumn{4}{|l|}{ BrS (3 months) } \\
\hline Ventricular-like $(\mathrm{n}=17)$ & $-68.0 \pm 1.1$ & $105.8 \pm 1.8$ & $16.9 \pm 1.1$ \\
\hline Atrial-like $(\mathrm{n}=1)$ & -70.2 & 101.4 & 21.5 \\
\hline Nodal-like $(\mathrm{n}=3)$ & $-53.1 \pm 1.5$ & $87.4 \pm 0.6$ & $2.3 \pm 0.1$ \\
\hline
\end{tabular}

Resting membrane potential (RMP), action potential amplitude (APA), maximal upstroke velocity $\left(\mathrm{V}_{\max }\right)$, number of cardiomyocytes (n). Data are presented as mean \pm SEM.

The $\mathrm{V}_{\max }$ is a measure of sodium channel availability in ventricular- and atrial-CMs. Due to the minor role of sodium channels contributing to the depolarization in nodal-like cells, this subtype was excluded from further AP analyses regarding the $\mathrm{V}_{\max }$ during depolarization. The $\mathrm{V}_{\max }$ values of atrial- and ventricular-like CMs were pooled and grouped into 1- and 3-month- 
old BrS- and Ctrl-CMs (Fig. 28). On average, the difference of $\mathrm{V}_{\max }$ in 1-month-old BrScompared to Ctrl-CMs was marginal (see also Table 12). However, a population with a reduced $\mathrm{V}_{\max }(\leq 10 \mathrm{~V} / \mathrm{s})$ in 1-month-old BrS-CMs was clearly detectable (Fig. 28). In 3month-old BrS-CMs, $\mathrm{V}_{\max }$ was significantly smaller than in Ctrl-CMs, with a subpopulation of cells where $\mathrm{V}_{\max }$ is $\leq 15 \mathrm{~V} / \mathrm{s}$. Additionally, the average $\mathrm{V}_{\max }$ was significantly increased in 3-month-old Ctrl-CMs compared to 1-month-old CMs (see also Table 12).

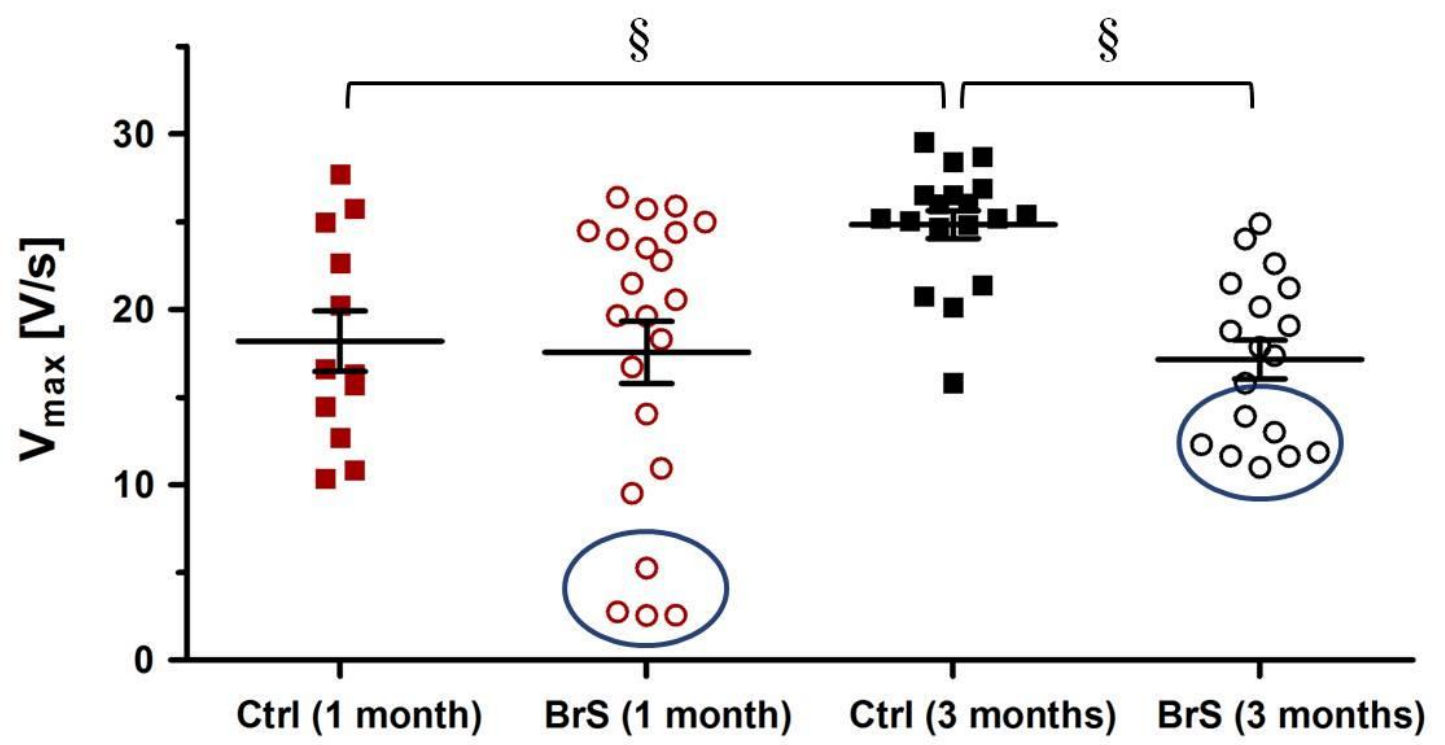

Figure 28. $\mathrm{V}_{\max }$ in atrial- and ventricular-like CMs of 1- and 3-month-old BrS- and Ctrl-CMs. On average, the difference of $\mathrm{V}_{\max }$ in 1-month-old BrS- compared to Ctrl-CMs was marginal, although a population of BrS-cells with $\mathrm{V}_{\max }<10 \mathrm{~V} / \mathrm{s}$ was visible (blue circle, left). In 3-month-old Ctrl-CMs the average $\mathrm{V}_{\max }$ was significantly increased compared to 1-month-old Ctrl-CMs. A significantly reduced $\mathrm{V}_{\max }$ in 3-monthold BrS-CMs compared to Ctrl-CMs was clearly detectable. Unpaired Student's $t$-test was used for statistical analysis. Statistical significance is represented by $(\S) \mathrm{p}<0.001$.

\subsubsection{BrS-CMs show increased AP irregularities and beat-to-beat variability of repolarization duration}

$\mathrm{BrS}$ is associated with increased incidences of ventricular tachycardia. EAD- and DADinduced triggered activity is capable of initiating cardiac arrhythmias. To further investigate the functional consequences of the reduced $I_{N a}$ in BrS-CMs and its impact on arrhythmias, irregularities during spontaneous AP recordings were studied compared to Ctrl-CMs. Three prominent irregularities were detected: EADs and EAD-induced triggered activity (Fig. 29C), DADs and DAD-induced triggered activity (Fig 29D), as well as beat-to-beat variability of repolarization durations of sequential APs (Fig. 29B). These irregularities were counted together as arrhythmogenic and compared between BrS- and Ctrl-CMs. In $35 \%$ of all recorded 1-month-old $\mathrm{BrS}-\mathrm{CMs}(\mathrm{n}=77)$ arrhythmic events were detectable, compared to 
$16 \%$ of all 1 -month-old Ctrl-CMs $(n=48)$. Among the 3 -month-old cells $44 \%$ of the BrSCMs $(n=27)$ showed arrthythmic events compared to $11 \%$ of the Ctrl-CMs $(n=45)$.

A
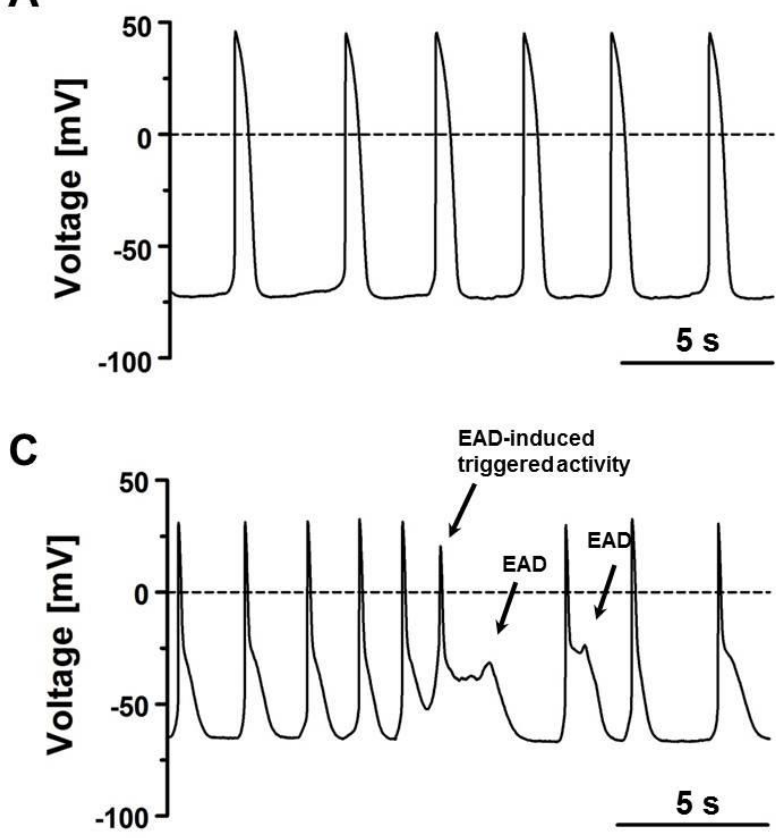

B

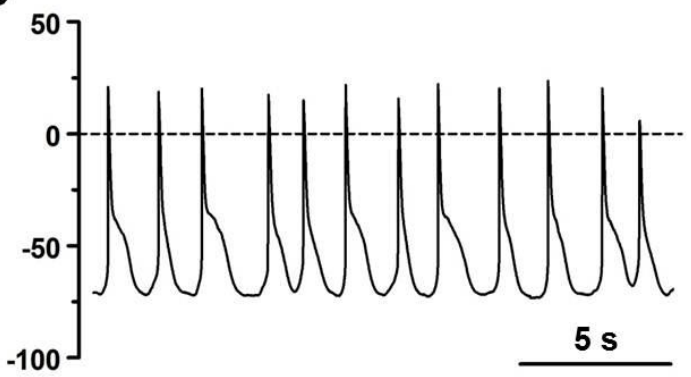

D

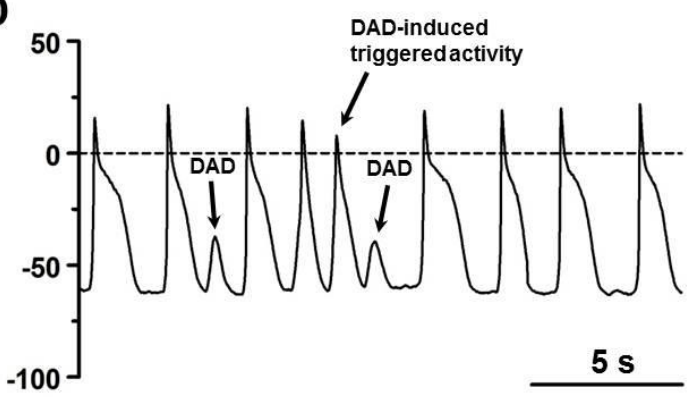

Figure 29. Traces of spontaneous AP recordings from Ctrl- (A) and BrS-CMs (B - D). The absolute majority of the Ctrl-CMs showed rhythmical AP recordings (A). Arrhythmical AP recordings were observed more often among the BrS-CMs. In many cases, APs showed a high APD variability (B). Additionally, EADs and EAD-induced triggered activity (C) and DADs and DAD-induced triggered activity (D) appeared spontaneously in BrS-CMs.

Electrophysiological recordings of spontaneously beating CMs already revealed a high beatto-beat variability of repolarization durations of sequential APs within one measurement. The APD is defined by the time needed for the repolarization of the cell. For example, APD90 defines the time, which is needed for $90 \%$ of the full repolarization of the cell. However, the APDs are dependent on the beating frequency. Moreover, it is well known that the clinical manifestation of $\mathrm{BrS}$ is dependent on the beating rate (Extramiana et al. 2006). Therefore, in order to compare the APDs between Ctrl- and BrS-CMs, the cells were paced at a physiological rate. In this study, the APs were recorded while the CMs were paced at two different frequencies $(0.8$ and $1.0 \mathrm{~Hz})$. The variability among sequential APs within one measurement was defined by the difference between the shortest and the longest APD (Fig. 30). The difference was calculated for APD50, APD70, and APD90. Patch clamp recordings in 1- as well as 3-month-old Ctrl-CMs demonstrated a relatively low beat-to-beat variability, when paced at 0.8 and $1.0 \mathrm{~Hz}$ (Fig. 30A). In contrast, BrS-CMs showed a high beat-to-beat variability (Fig. 30B). 
A
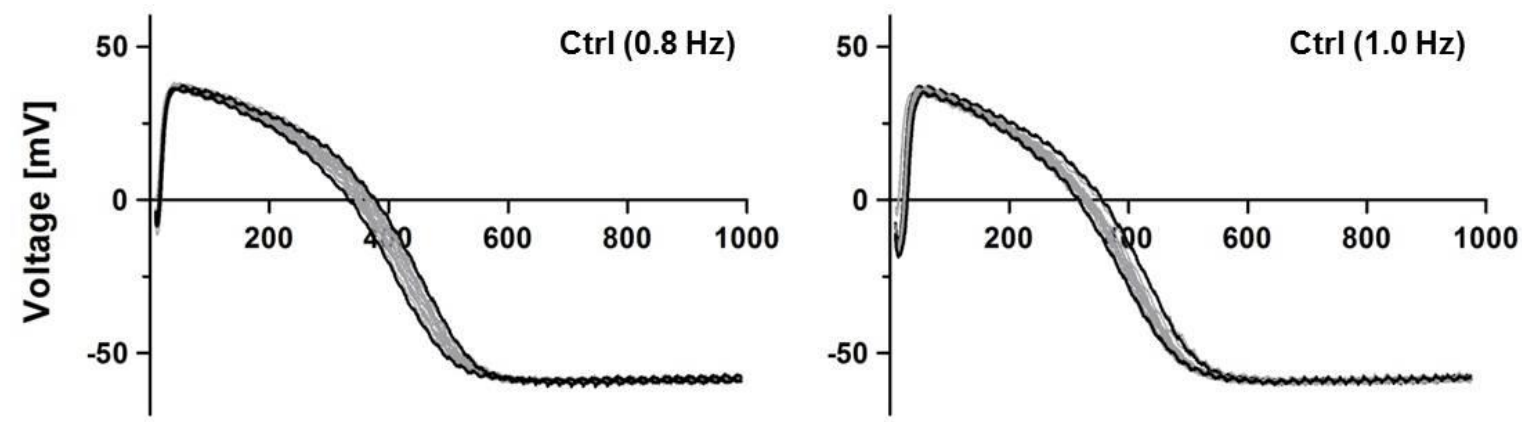

B
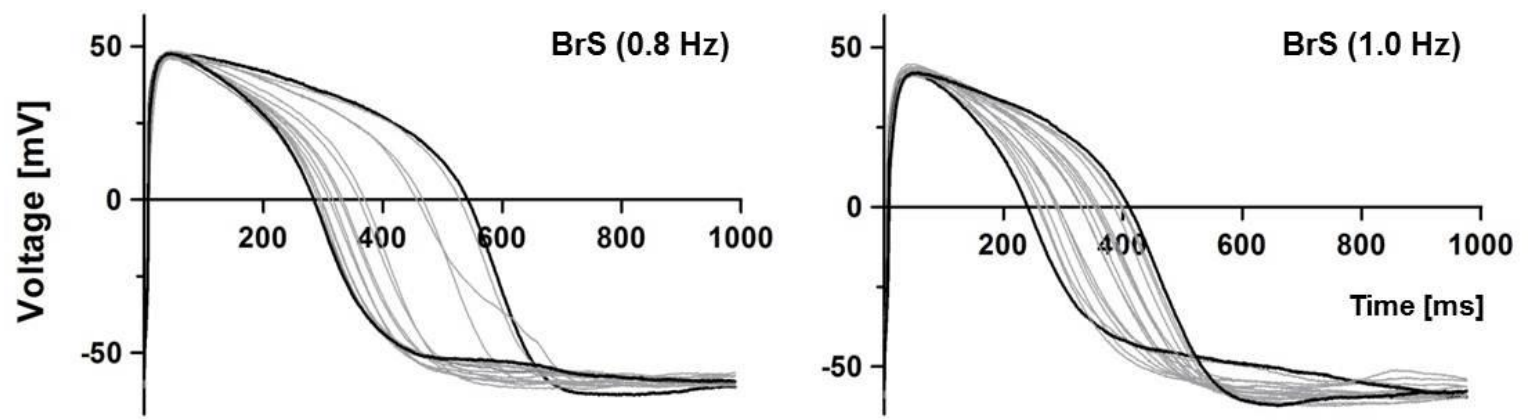

Figure 30. APD variability in Ctrl- and BrS-CMs. When electrically paced with 0.8 or $1.0 \mathrm{~Hz}$, Ctrl-CMs showed little beat-to-beat variability of sequential APs regarding the duration of $50 \%, 70 \%$, and $90 \%$ of repolarization (shortest and longest AP in black (A)). In contrast, BrS-CMs showed a significantly increased beat-to-beat variability compared to Ctrl-CMs (B).

When paced at 0.8 and $1.0 \mathrm{~Hz}$, the variability of APD50, APD70, and APD90 was significantly increased in both 1- and 3-month-old BrS-CMs compared to the respective CtrlCMs (Fig. 31A-D). 
A

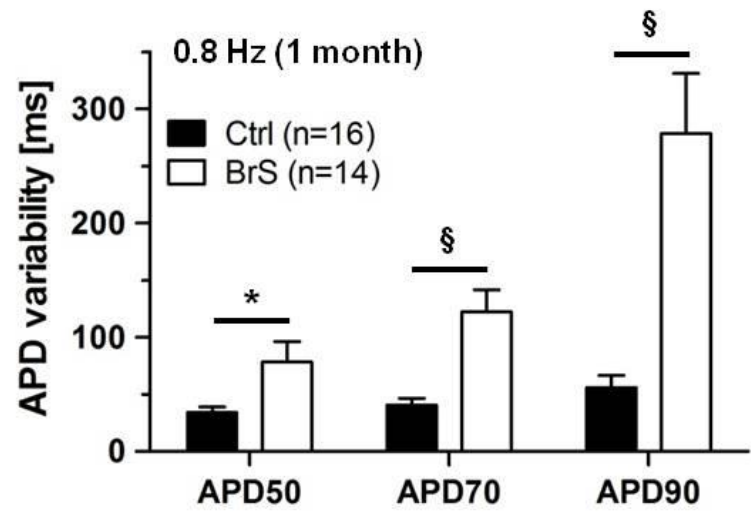

C

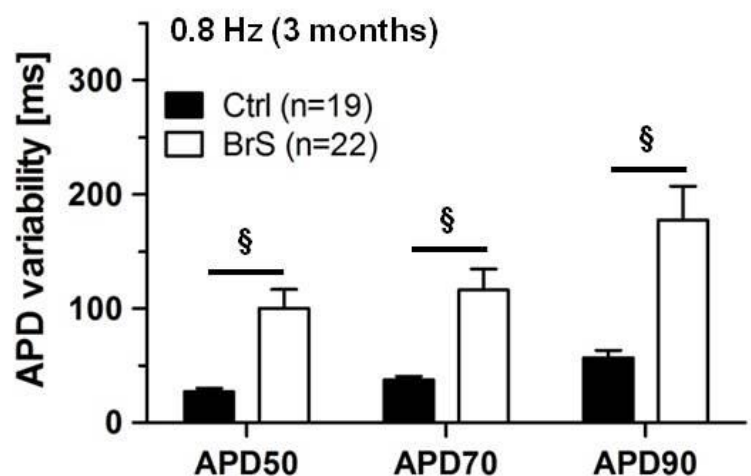

B

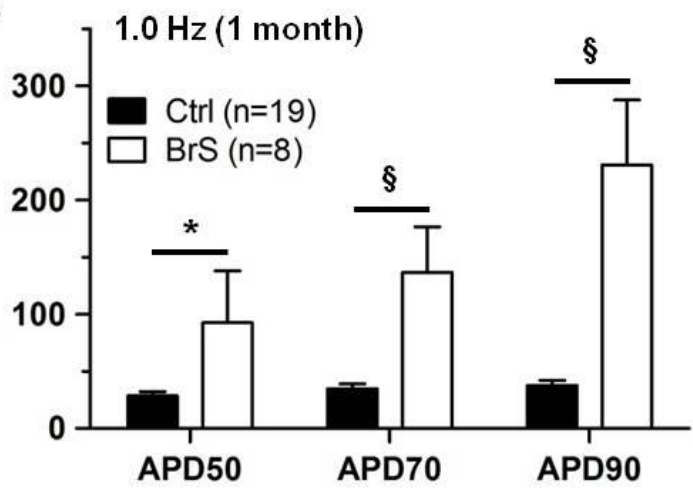

D

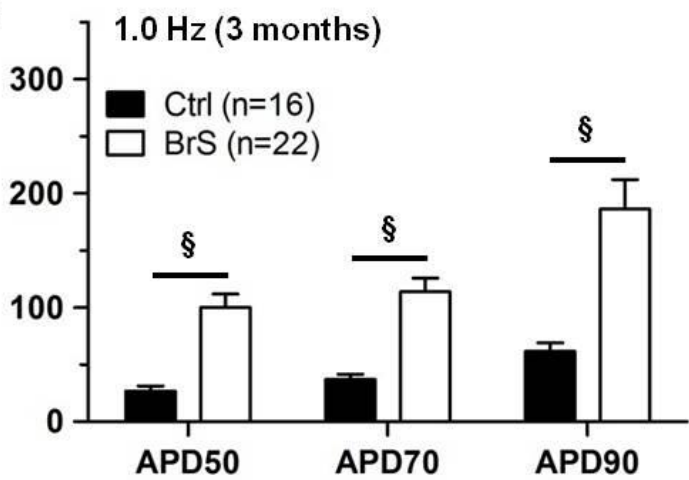

Figure 31. Quantitative analysis of the beat-to-beat APD variability in 1- and 3-month-old Ctrl- and BrSCMs. For the calculation of the variability, the shortest APD of one measurement was substracted from the longest APD for APD50, APD70, and APD90. (A) 1 month $(0.8 \mathrm{~Hz})$, (B) 1 month $(1.0 \mathrm{~Hz}),(\mathbf{C}) 3$ months $(0.8 \mathrm{~Hz})$, and $(\mathbf{D}) 3$ months $(1.0 \mathrm{~Hz})$. The variability of APD50, APD70, and APD90 was significantly increased in BrS-CMs compared to Ctrl-CMs measured at 0.8 and $1.0 \mathrm{~Hz}$ for both 1- and 3-month-old cells. Data are presented as mean \pm SEM. Unpaired Student's $t$-test was used for statistical analysis. Statistical significance is represented by $\left.{ }^{*}\right) \mathrm{p}<0.05$ and $(\S) \mathrm{p}<0.001$.

\subsubsection{Sodium channel blocker flecainide induces increased APD variability in Ctrl-CMs}

To find out whether the increased APD variability in BrS-CMs is correlated to the reduced $I_{N a}, 3$-month-old Ctrl-CMs were treated with the sodium channel blocker flecainide. $I_{N a}$ and APs were recorded. When the Ctrl-CMs were treated with flecainide at a concentration of $2.5 \mu \mathrm{M}$, a reduction of the average $I_{N a}$ density similar to that of 3-month-old BrS-CMs was observed (see Fig. 22C). Furthermore, a slight right-shift of the I-V curve was also detected, indicating delayed sodium channel activation (Fig. 32A). The peak $I_{N a}$ density in Ctrl-CMs treated with flecainide was significantly reduced to $50 \%$ (Fig. 32B) and appeared at a more positive potential $(-30 \mathrm{mV})$ compared to Ctrl-CMs without treatment $(-35 \mathrm{mV})$. 
A

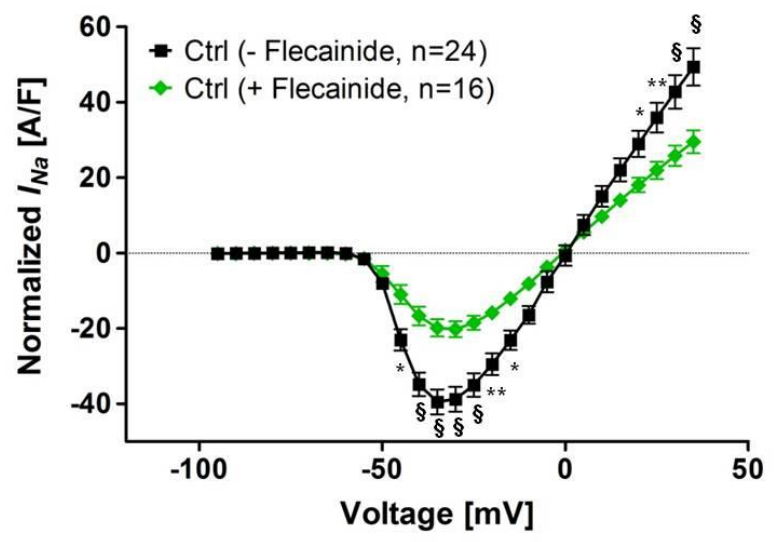

B

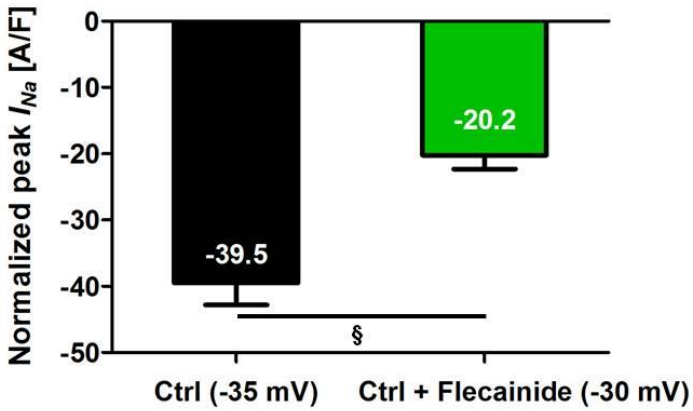

Figure 32. $I_{N a}$ measurements in Ctrl-CMs treated with flecainide. 3-month-old Ctrl-CMs were treated with $2.5 \mu \mathrm{M}$ of the sodium channel blocker flecainide. The I-V curve of Ctrl-CMs treated with flecainide showed a significant reduced $I_{\mathrm{Na}}$ and a slight right shift compared to untreated Ctrl-CMs (A). Peak current density in CtrlCMs with flecainide (at $-30 \mathrm{mV}$ ) was significantly reduced to $50 \%$ in comparison to Ctrl-CMs without treatment (at $-35 \mathrm{mV})(\mathbf{B})$. Data are presented as mean \pm SEM. Two-way repeated measures ANOVA for I-V curves and unpaired Student's $t$-test for peak $I_{N a}$ densities were used for statistical analysis: $(*) \mathrm{p}<0.05$, $(* *) \mathrm{p}<0.01,(\S) \mathrm{p}<0.001$

AP recordings during pacing at $0.8 \mathrm{~Hz}$ (Fig. 33A) and $1.0 \mathrm{~Hz}$ (Fig. 33B) demonstrated a significantly increased variability of APD50, APD70, and APD90 when the cells were treated with flecainide. These data indicate a correlation of the reduced $I_{N a}$ and the APD variability.

A

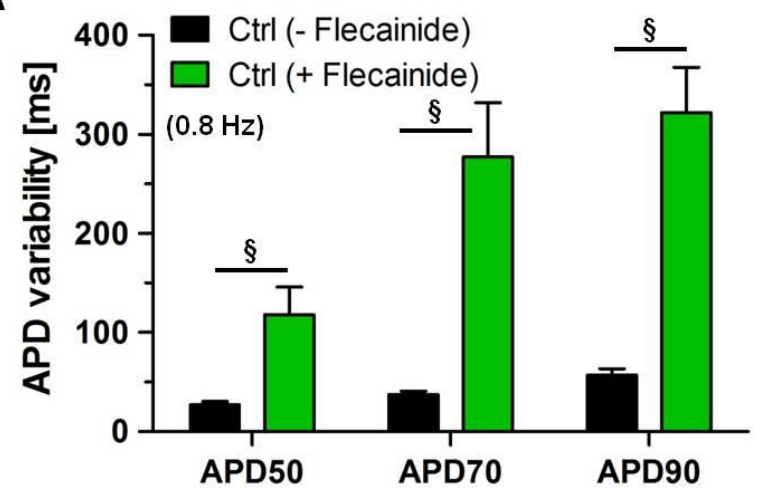

B

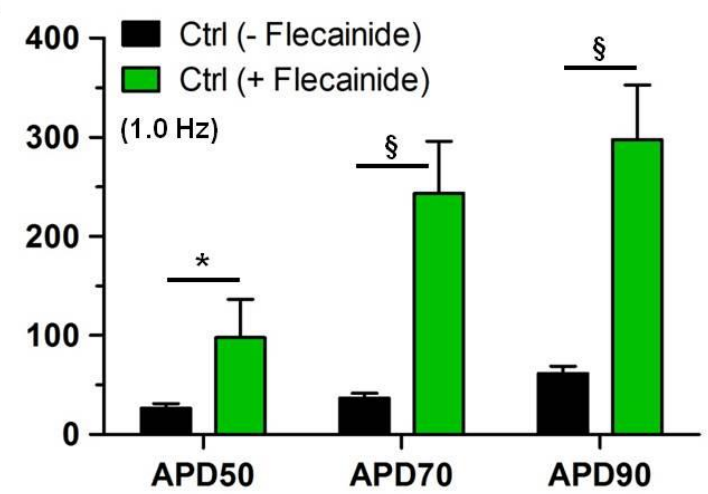

Figure 33. Beat-to-beat APD variability in Ctrl-CMs treated with flecainide. The APs of paced Ctrl-CMs at $0.8 \mathrm{~Hz}(\mathbf{A})$ and $1.0 \mathrm{~Hz}(\mathbf{B})$ showed a significantly increased beat-to-beat variability when treated with flecainide. Ctrl (- flecainide): $\mathrm{n}=16, \mathrm{Ctrl}$ (+ flecainide): $\mathrm{n}=9$. Data are presented as mean \pm SEM. Student's $t$-test was used for statistical analysis. Statistical significance is represented by $\left(^{*}\right) \mathrm{p}<0.05$, and $(\S) \mathrm{p}<0.001$.

\subsubsection{Antiarrhythmic agent quinidine does not decrease APD variability in BrS-CMs}

Quinidine is a class I antiarrhythmic agent which is currently used in clinical trials for the treatment of BrS patients. To study the influence of quinidine on APD variability in BrSCMs, the cells were treated with the drug at concentration of $5 \mu \mathrm{M}$. AP measurements showed 
a prolongation of the APD and a slower spontaneous beating frequency (Fig. 34A). Pacing experiments could not be performed under previous conditions (pacing frequencies of 0.8 and $1.0 \mathrm{~Hz}$ ) because of the increased APD under quinidine treatment. When paced at 0.8 or $1.0 \mathrm{~Hz}$, the cells were not able to repolarize completely before the next stimulus. Therefore, the cells were paced at $0.4 \mathrm{~Hz}$ to allow for complete repolarization. However, AP recordings with lower pacing frequencies still revealed a high beat-to-beat APD variability in BrS-CMs, indicating that quinidine has no effect on the APD variability (Fig. 34B).

A

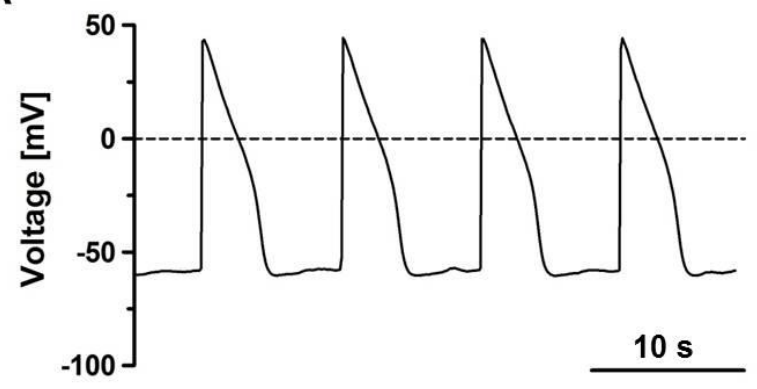

B

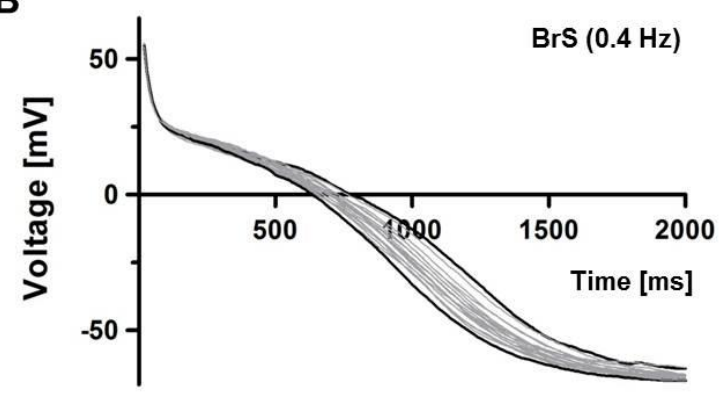

Figure 34. AP recordings of BrS-CMs under quinidine treatment. Spontaneous AP measurements showed a prolongation of the APD and a slower spontaneous beating frequency (A). AP recordings with low pacing frequencies $(0.4 \mathrm{~Hz})$ still revealed a high beat-to-beat APD variability in BrS-CMs (shortest and longest AP in black (B)).

\subsection{Role of SCN5A mutation C5435A (p.S1812X) in the pathogenesis of BrS}

\subsubsection{Expression of SCN5A in BrS-CMs on mRNA level}

To study the total SCN5A gene expression in BrS-CMs in comparison to Ctrl-CMs, quantitative real-time PCR was performed. Three-month-old CMs from spontaneous and twomonth-old CMs from directed differentiations were analyzed. Since a $100 \%$ purity of CMs in the samples cannot be guaranteed, the SCN5A expression was normalized to the early cardiac differentiation marker $c T N T$ and the late cardiac differentiation marker CASQ2. The qPCR data revealed a higher SCN5A expression in BrS-CMs compared to Ctrl-CMs when normalized to $c T N T$ (1.6-fold) and to CASQ2 (2-fold). However, the higher expression level was not significant. Additionally, the expression level of CASQ2 when normalized to $c T N T$ showed no significant difference between BrS- and Ctrl-CMs (Fig. 35). These data indicate no significant maturation differences between BrS- and Ctrl-CMs. 


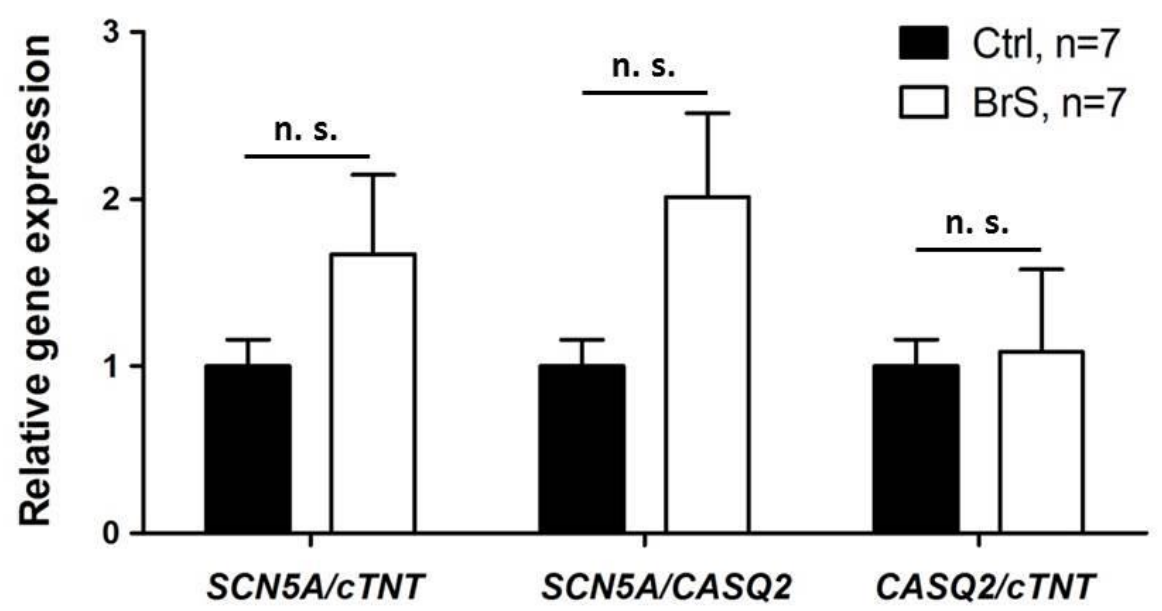

Figure 35. Total SCN5A gene expression in Ctrl- and BrS-CMs. On average, qPCR analyses showed a higher SCN5A expression in BrS-CMs compared to Ctrl-CMs when normalized to cTNT (1.6-fold) as well as to CASQ2 (2-fold). However, the difference was not significant (tested with unpaired Student's $t$-test, n. s. = not significant). The CASQ2 expression normalized to $c T N T$ was not altered in BrS-CMs compared to Ctrl-CMs. Data are presented as mean $\pm \mathrm{SEM}$.

One allele of the SCN5A gene of the BrS patient is characterized by the point mutation C5435A in exon 28. To study whether an allelic expression imbalance of SCN5A exists in $\mathrm{BrS}-\mathrm{CMs}$, the allele-specific expression of the gene was determined with the Ion Torrent ${ }^{\mathrm{TM}}$ semiconductor sequencing system. Total mRNA of 3-month-old beating clusters and 2month-old selected CMs from one directed cardiac differentiation experiment were collected, reverse transcribed into cDNA and the region which included the mutation was amplified and subsequently sequenced. In addition, genomic DNA (gDNA) from differentiated hiPSCs of the BrS patient was sequenced as control. The results showed no significant difference of the gene expression level of the wildtype (wt, $47.8 \pm 0.32 \%$ ) allele compared to the mutated (C5435A, 52.2 $\pm 0.31 \%$ ) allele (Fig. 36). Sequencing of the gDNA revealed similar copy numbers of the wt allele $(52.2 \pm 0.29 \%)$ and mutated allele $(47.8 \pm 0.29 \%)$ in the hiPSCderived $\mathrm{CMs}$ of the $\mathrm{BrS}$ patient. 


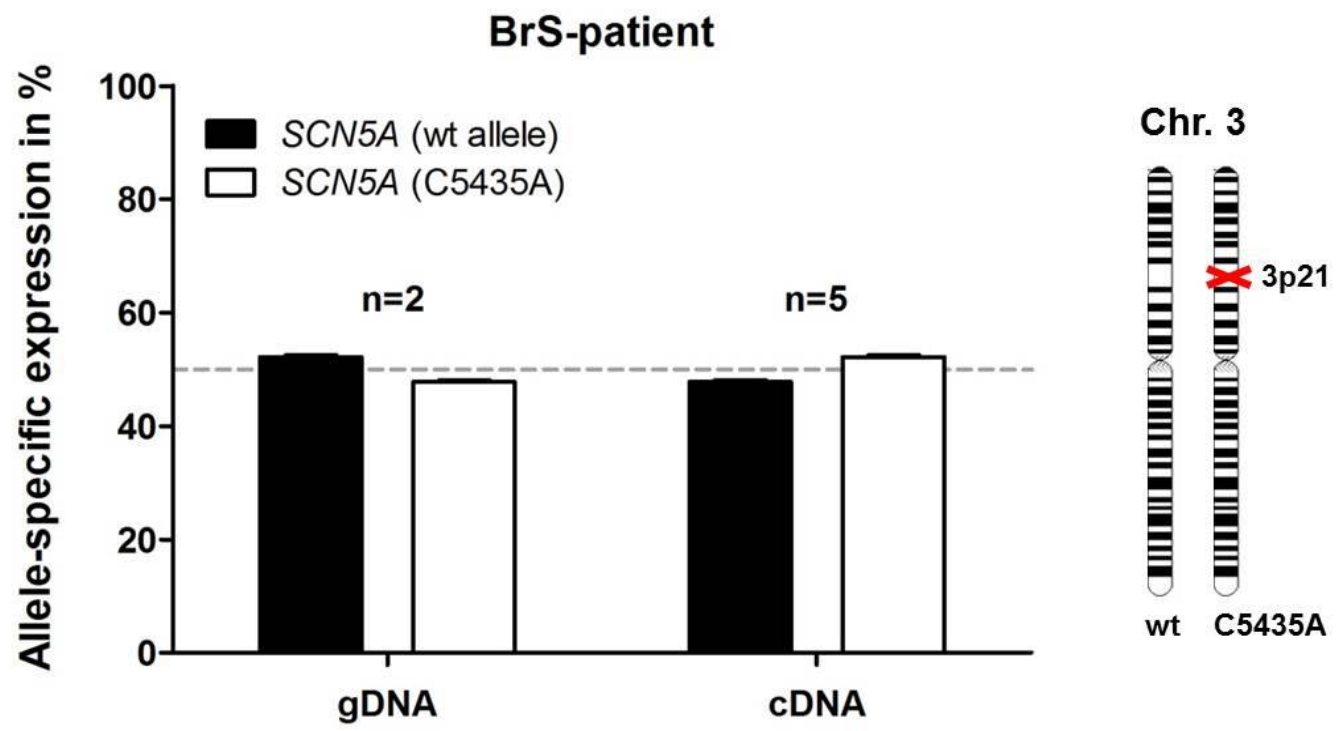

Figure 36. Allele-specific SCN5A expression in BrS-CMs. The sequencing of cDNA copies revealed no significant difference in the expression level of the wt $(47.8 \pm 0.32 \%)$ compared to the mutated $(52.2 \pm 0.31 \%)$ allele. The gDNA sequencing showed similar copy numbers of the wt allele $(52.2 \pm 0.29 \%)$ compared to the mutated allele $(47.8 \pm 0.29 \%)$. Data are presented as mean \pm SEM.

\subsubsection{Expression of Nav1.5 protein in BrS-CMs}

To further investigate the functional consequences of the SCN5A mutation C5435A (p.S1812X) in BrS-CMs, the cellular distribution of the Nav1.5 channels was first examined using an antibody against the total protein of Nav1.5 (Fig. 37). Immunostaining showed that the Nav1.5 channels were mainly expressed in a diffused or a fine granular network-like pattern in hiPSC-derived CMs. A similar distribution of the Nav1.5 channels in Ctrl-CMs compared to BrS-CMs was found.
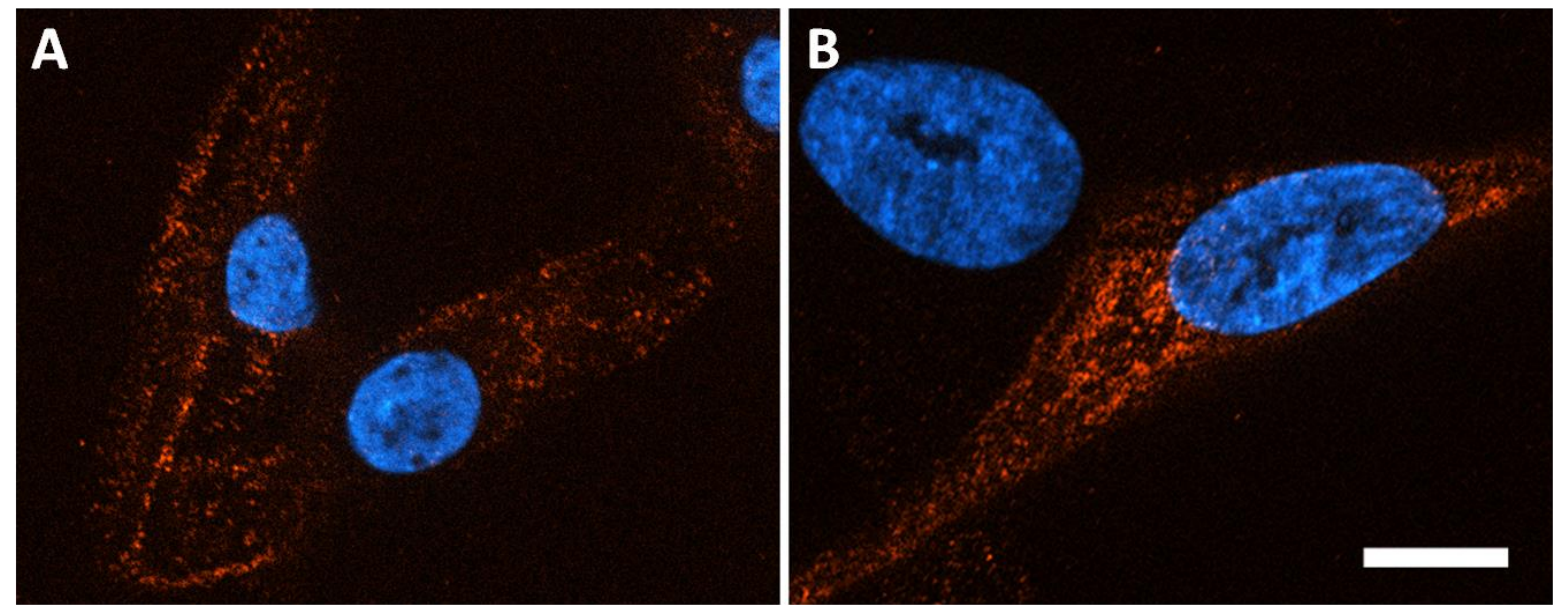

Figure 37. Immunostaining of hiPSC-derived CMs detecting Nav1.5. Ctrl-CMs (A) and BrS-CMs (B) showed a similar distribution of $\mathrm{Na}_{\mathrm{V}} 1.5$. Cell nuclei were stained with DAPI (in blue). Scale bar: $10 \mu \mathrm{m}$. 
As mentioned before, the SCN5A mutation C5435A (p.S1812X) is expected to give rise to a truncated $\mathrm{Na}_{\mathrm{v}} 1.5$ protein. As the patient is heterozygous for this mutation, it is expected that $50 \%$ of the functional, full-length protein is translated. To address the question whether the significantly reduced $I_{N a}$ of about $50 \%$ in BrS-CMs is due to nonfunctional sodium channels or due to less sodium channels integrated into the cell membrane, Western blot analyses with anti-Nav1.5 antibodies binding at different epitopes of the channel were performed. Four different antibodies were tested: three antibodies against the total $\mathrm{Na}_{\mathrm{V}} 1.5$ protein in both Ctrland $\mathrm{BrS}-\mathrm{CM}$ s (anti-human-Nav1.5 detecting an extracellular domain (1), anti-mouse-Nav1.5 detecting amino acid residues 493 - 511 with 17/19 identical with human (2), anti-rat-Nav1.5 detecting amino acid residues 493 - 511 with 17/19 identical with human (3)) and one against only the full length protein which binds on the C-terminus of the protein (anti-human-Nav1.5 detecting amino acid residues 1978 - 2016). Only the latter showed specific results (Fig. 38).
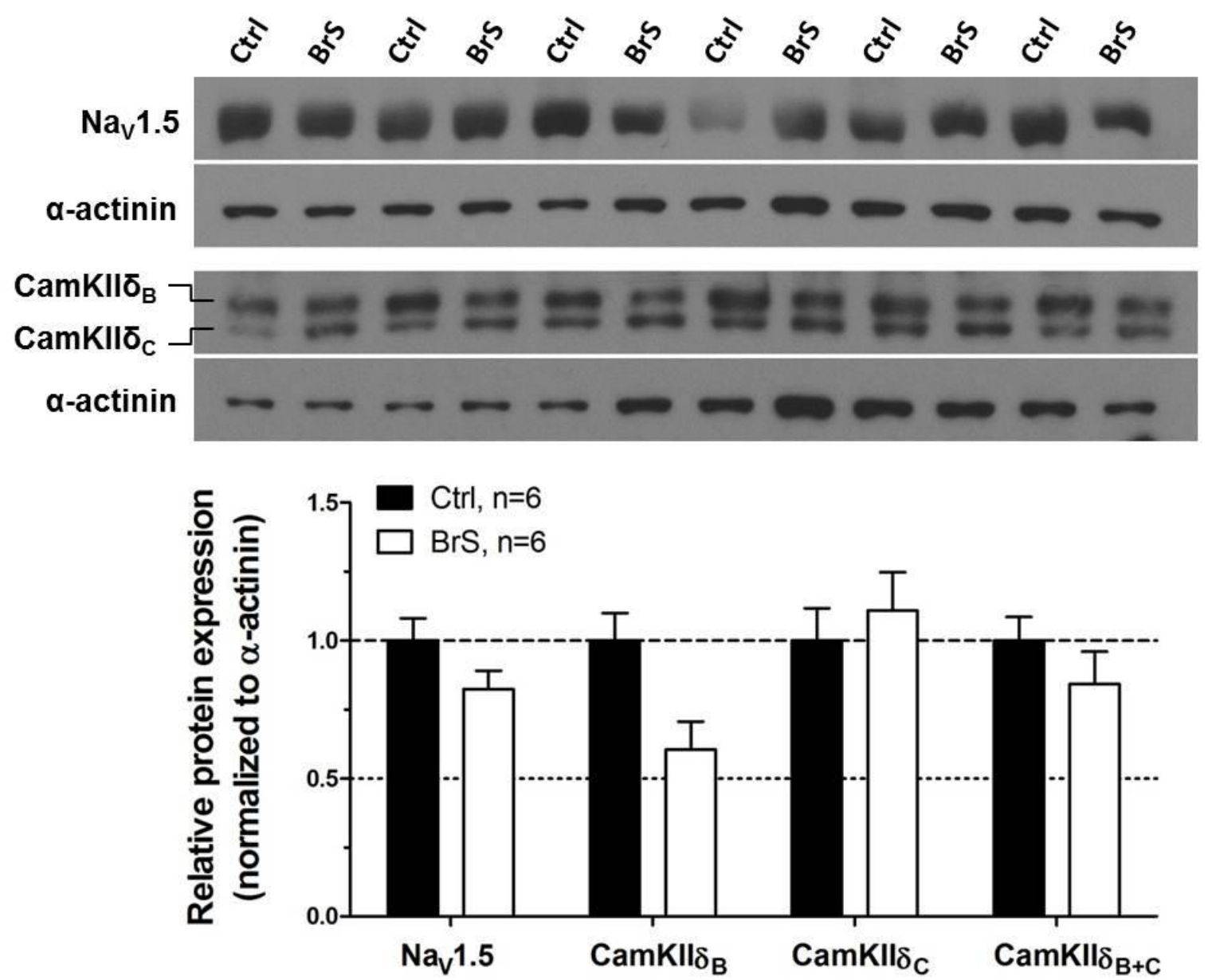

Figure 38. Western blot analyses in BrS- ad Ctrl-CMs. Antibodies detecting the full-length protein $\mathrm{Na}_{\mathrm{V}} 1.5$ demonstrated $82 \%$ expression in BrS- compared to Ctrl-CMs, indicating an upregulation of the full-length protein in BrS-CMs. In BrS-CMs, both isoforms of $\mathrm{Ca}^{2+} /$ calmodulin-dependent protein kinase II- $\delta$ (CamKII $\delta$ ) together contributed to $84 \%$ of the expression level in Ctrl-CMs. The isoform CamKII $\delta_{\mathrm{B}}$ was $40 \%$ downregulated in BrS-CMs, whereas $\mathrm{CamKII}_{\mathrm{C}}$ was slightly higher expressed compared to that in Ctrl-CMs. All proteins were normalized to $\alpha$-actinin. 
The Western blot results showed that the expression of the full-length $\mathrm{Na}_{\mathrm{V}} 1.5$ protein in $\mathrm{BrS}$ CMs was reduced by $18 \%$ when normalized to $\alpha$-actinin and compared to Ctrl-CMs.

Previous studies showed that $\mathrm{Ca}^{2+} /$ calmodulin-dependent protein kinase II- $\delta$ (CamKII $\delta$ ) could modulate $\mathrm{Na}_{\mathrm{V}} 1.5$ and was involved in regulation of $I_{N a}$ (Ashpole et al. 2012). Therefore, the expression level of the predominant cardiac isoform CamKII $\delta_{\mathrm{C}}$, which is part of the macromolecular complex surrounding $\mathrm{Na}_{\mathrm{v}} 1.5$, was studied in Ctrl- and BrS-CMs. The antibody used in this Western experiment recognizes both isoforms CamKII- $\delta_{\mathrm{B}}$ and CamKII $\delta_{\mathrm{C}}$. The data showed that the average expression of both CamKII $\delta$ isoforms together was $16 \%$ reduced in BrS-CMs compared to Ctrl-CMs when normalized to $\alpha$-actinin. A similar expression relationship was observed for $\mathrm{Na}_{\mathrm{v}} 1.5$ between $\mathrm{BrS}$ - and Ctrl-CMs (Fig. 36). The isoform $\mathrm{CamKII}_{\mathrm{B}}$ was $40 \%$ downregulated in $\mathrm{BrS}-\mathrm{CMs}$, whereas the expression level of CamKII $\delta_{C}$ was slightly higher in comparison to that in Ctrl-CMs (Fig. 36).

Taken together, SCN5A expression analyses revealed a slight upregulation on mRNA level, with a balance in allele-specific expression. The translation of full-length $\mathrm{Na}_{\vee} 1.5$ protein was however reduced by only $18 \%$ in BrS-CMs compared to Ctrl-CMs, although the expression level was expected at $50 \%$. 


\section{Discussion}

The Brugada syndrome is a distinct subgroup of idiopathic ventricular tachycardia or ventricular fibrillation and is one of the major causes of sudden cardiac death in healthy young people. However, the underlying electrophysiological and molecular mechanisms have not been completely understood. A big challenge in cardiac translational research is the lack of tissue culture systems recapitulating human pathology to study disease mechanisms and to identify druggable targets. The discovery of inducing pluripotency by the ectopic expression of certain transcription factors in somatic cells represents a milestone in medical research (Takahashi and Yamanaka 2006). Patient-specific hiPSCs as a renewable and unlimited source for CMs provide the possibility to study the pathophysiology of genetic diseases in vitro.

The aim of this thesis was to investigate whether CMs differentiated from hiPSCs generated from hMSCs of a BrS patient could recapitulate electrophysiological features of the disease in vitro as well as to study the underlying molecular mechanisms. Firstly, hiPSCs were generated from a 45-year-old female healthy control and a 50-year-old male BrS patient heterozygous for a point mutation (C5435A) in the SCN5A gene, encoding for the $\alpha$-subunit of the cardiac sodium channel $\mathrm{Na}_{\vee} 1.5$.

The generated Ctrl- and BrS-iPSCs exhibited hESC-like characteristics, demonstrated by pluripotency related gene expression, demethylation status in the promoter regions of NANOG and OCT4, in vitro differentiation capacity, and teratoma formation. Furthermore, both Ctrland BrS-hiPSCs were able to differentiate into functional CMs, which were shown by spontaneous contraction, drug response and cardiac-specific protein expression. Electrophysiological analyses showed a loss-of-function of the sodium channels with a delayed activation in BrS-CMs compared to Ctrl-CMs. Steady-state inactivation and the recovery from inactivation of sodium channels in BrS-CMs were comparable to Ctrl-CMs, whereas the intermediate inactivation of cardiac sodium channels was slightly, but not significantly enhanced in BrS-CMs. AP measurements revealed a reduced $\mathrm{V}_{\max }$ and higher arrhythmic tendencies in BrS-CMs, mainly expressed as an increased beat-to-beat APD variability.

Total SCN5A expression on mRNA level was found to be slightly upregulated in BrS-CMs compared to Ctrl-CMs, albeit showing allele balanced expression. On protein level, fulllength $\mathrm{Na}_{\mathrm{v}} 1.5$ expression was reduced by $18 \%$ in BrS-CMs compared to Ctrl-CMs. 


\subsection{Successful generation of human iPSCs}

In this study, the STEMCCA lentivirus system was used for the reprogramming of Ctrl- and BrS-hMSCs into hiPSCs. STEMCCA is single polycistronic "stem cell cassette" containing all four Yamanaka factors $O C T 4, S O X 2, K L F 4$, and $c-M Y C$, separated by self-cleaving $2 \mathrm{~A}$ peptide and IRES sequences (Somers et al. 2010). The combination of 2A peptide and IRES elements allows for the production of the four individual transcription factors (Ibrahimi et al. 2009). The simultaneous expression of the four transcription factors is driven by a constitutive EF-1 $\alpha$ promoter on a single vector, which leads to a reduction of viral integration sites and a higher reprogramming efficiency compared to the use of four single vectors (Somers et al. 2010; Streckfuss-Bömeke et al. 2013). However, STEMCCA integrates randomly into the genome, which might disrupt important genetic information and increase the risk of insertional mutagenesis. Even after excision of STEMCCA using the Cre/LoxP system, around $200 \mathrm{bp}$ of an inactive viral long terminal repeat site remains in the host genome (Somers et al. 2010). There are continuing efforts in the development of transgene-free reprogramming methods with high efficiencies (Durruthy-Durruthy et al. 2014).

The molecular mechanisms behind the process of cellular reprogramming have not been completely understood yet. In the present study, ectopic expression of the four Yamanaka factors (Takahashi and Yamanaka 2006) in hMSCs resulted in the successful generation of hiPSCs. The homeodomain transcription factor of the POU family Oct4 is expressed in the nuclei of early blastomeres (Rosner et al. 1990). During development, its expression becomes restricted to the pluripotent inner cell mass and later to the primordial germ cells and finally to oocytes (Pesce et al. 1998). Oct4 dimerizes with Sox2 to form a transcription complex, which activates their own gene expression in a positive feedback (Masui et al. 2007; Tomioka et al. 2002). Furthermore, Oct4 seems to inhibit the transcription of the microRNA miR145, which would on its part block the translation of Oct4, Sox2, and Klf4 (Chivukula and Mendell 2009; $\mathrm{Xu}$ et al. 2009). These transcription factors in turn activate other transcription factors important for inducing pluripotency, for example, Nanog (Rodda et al. 2005). Nanog, a homeodomain protein is essential for the maintenance of self-renewal in ESCs (Chambers et al. 2003; Mitsui et al. 2003). Importantly, previous studies show that human OCT4, SOX2, and NANOG are central to the transcriptional network and regulate actively transcribed genes, including transcription factors and signaling components necessary to maintain the pluripotent state of hESCs (Boyer et al. 2005). 
These genes, including others like LIN28, FOXD3, GDF3, SSEA4, or TRA-1-60 are found to be highly expressed in undifferentiated hESCs, whereas their downregulation is linked to differentiation (Henderson et al. 2002; Sato et al. 2003; Richards et al. 2004; Bhattacharya et al. 2004; Clark et al. 2004; Levine and Brivanlou 2005; Hyslop et al. 2005; International Stem Cell Initiative 2007). The generated hiPSCs of the present study showed hESC-like characteristics with regards to their morphology and expression of pluripotency related markers, including OCT4, SOX2, NANOG, LIN28, FOXD3, GDF3, SSEA4, or TRA-1-60, indicating that the ectopic expression of OCT4, SOX2, KLF4, and $c-M Y C$ in hMSCs initiated the activation of pluripotency related endogenous genes (Boyer et al. 2005; Kim et al. 2008). This is preceded by alterations of the epigenome (epigenomic reprogramming), as demonstrated by the demethylation state of the analyzed promoter regions of NANOG and OCT4 in the generated hiPSCs.

Furthermore, upon in vitro differentiation of both Ctrl- and BrS-iPSCs the cells expressed markers specific for all three germ layers, demonstrating that they are truly pluripotent. The ability to differentiate into almost all tissue types is the hallmark of human pluripotent stem cells (Itskovitz-Eldor et al. 2000). Teratoma formation assays are considered to be the "gold standard" for demonstrating the differentiation potential of human pluripotent stem cells (Zhang et al. 2008). In vivo differentiation of Ctrl-hiPSCs revealed tissue-specific cells including all three germ layers, such as cartilage and muscle cells (mesoderm), neural rosettes (ectoderm) and intestinal tissue (endoderm). BrS-hiPSCs formed teratomas containing endodermal and mesodermal cell, but no ectodermal cells. However, gene expression analyses showed the expression of ectodermal markers SYP and TH on RNA level as well as III $\beta$ tubulin on protein level, indicating the ability of BrS-hiPSCs to differentiate into the ectodermal lineage.

The evaluation of a normal diploid karyotype in hiPSCs is important for both basic research and future clinical use. In this study, hiPSCs up to a maximum of 30 passages were used for differentiation experiments to reduce the risk of the accumulation of chromosomal aberrations. Karyotype analyses showed that the majority (> $90 \%$ ) of both Ctrl- and BrShiPSCs (at passage $\geq 30$ ) exhibited a normal diploid karyotype. Only a few counted karyotypes showed 45 or less chromosomes. Adaptive partial and full chromosomal abnormalities have already been reported in various hESC lines after long-term cultivation, such as isodicentric X chromosomes (Inzunza et al. 2004) or gains of chromosomes 12 and 17q (Draper et al. 2004). The authors assumed that the increased dosage of the genes located 
on chromosomes 12 and 17 provided a selective advantage for the propagation of undifferentiated cells (Draper et al. 2004). The accumulation of either entire chromosome 12 or part of the short arm 12p during long-term culture was also found in some of hiPSC lines reported previously (Mayshar et al. 2010). Mayshar and colleagues identified in 13 out of 66 analyzed hiPSC lines chromosomal abnormalities, with 6 of them carrying at least one full trisomy (Mayshar et al. 2010). In addition, they found an abnormally high frequency of overexpressed genes on chromosome 12 at early passages, suggesting a remarkable selective pressure during the reprogramming process. The gain of chromosome 12 was shown to be associated with a significant overexpression of pluripotent and cell cycle-related genes, such as NANOG and GDF3 compared to other hiPSC lines carrying a diploid karyotype (Mayshar et al. 2010). Chromosomal aberrations adapted during culture conditions may limit the differentiation capacity of the hiPSCs (Enver et al. 2005) and may increase their tumorigenicity (Blum and Benvenisty 2009). Furthermore, such aberrations might also influence the interpretation of biological and disease-related studies of hiPSCs. In this study, a trisomy of chromosome 12 or 17 was not detected in the generated hiPSCs. So far, there is no literature reporting a loss of entire chromosomes in hESCs or hiPSCs during long-term cultivation. Thus, it is very likely that the loss of one or more chromosomes in some of the analyzed cells in this study was due to technical difficulties in preparations.

However, the karyotyping method in this study has some limitations. Structural chromosomal aberrations including insertions, deletions, or translocations of genetic material cannot be detected. Other methods such as fluorescence in-situ hybridization, spectral karyotyping, high-density comparative genomic hybridization arrays, or global gene expression metaanalysis allow the detection of structural chromosomal abnormalities at a higher resolution than standard karyotyping and should be considered for future chromosomal analyses.

Interestingly, sequencing analyses of genomic BrS- and Ctrl-hiPSCs revealed that both donors are carrier of a synonymous SNP on position 5457, with a cytosine on the one allele and a thymine on the other. Both variants lead to a codon for aspartic acid (D1819D). This SNP was already described within the Han Chinese population with a frequency of $41.3 \%$, in $46 \%$ of the Japanese, and in $12.3 \%$ of the American population (ethnicities not specified; Wattanasirichaigoon et al. 1999; Iwasa et al. 2000; Chen et al. 2004). The genotype and allele frequencies showed no significant difference when the control group was compared to $\mathrm{BrS}$ patients, demonstrating that this SNP is unlikely to be linked with BrS (Chen et al. 2004). 
Taken together, the generated patient-specific hiPSCs have similar characteristics as hESCs and fulfill the criteria defining fully reprogrammed hiPSCs with a stable karyotype. Because of their origin (somatic cells), the further use for research purposes does not raise underlying ethical concerns as hESCs do. In addition, to investigate the disease-specific phenotype of the mutation of interest (in the case of this study, the C5435A mutation in SCN5A) using hESCs, the mutation has to be inserted artificially. Moreover, the genetic background of hESCs differs from the BrS patient. Therefore, patient-specific hiPSCs carrying the mutation are more suitable for BrS disease modelling than hESCs.

\section{2 hiPSC-derived CMs are functional}

In the present study, both EB formation and directed cardiac differentiation methods were used for the generation of functional CMs. The EB method can also be referred as spontaneous differentiation method because no lineage-specific stimulus is applied during differentiation. Using the EB method, the generated hiPSCs, similar to hESCs, give rise to CMs at a rather low and variable differentiation efficiency (Kehat et al. 2001). About $8-42 \%$ of EBs contained beating clusters and lower than $5 \%$ of total cells were CMs. In addition, CMs generated with this method have to be manually isolated from the culture for further molecular and biochemical analyses, a laborious and time consuming task. To produce sufficient CMs for biochemical analyses, upscaling CM formation from hiPSCs was essential. In this study, the directed cardiac differentiation method (Lian et al. 2012; Lian et al. 2013) was combined with a metabolic-based cardiac selection (removal of glucose and application of lactate; Tohyama et al. 2013) for the generation of large quantities and a high purity (>90\%) of CMs. Together with CMs obtained from EB differentiation method, these cells were also used for SCN5A gene expression analyses. No significant difference in total and allele-specific $S C N 5 A$ expression between spontaneously and directed differentiated CMs was observed, indicating the generated CMs from the two methods are comparable. Therefore, the analyzed data were pooled. Cells obtained with the directed differentiation method were used for Western blot analyses as high quantity of cells were needed for sufficient protein isolation.

The contractile properties of both Ctrl- and BrS-CMs were similar to those derived from hESCs, and did not differ significantly among all analyzed hiPSC lines. The present data showed that the hiPSC-derived CMs have the complex functional properties of native CMs, including responses to L-type calcium channel blocker Diltiazem and activator Bay K8644. The hiPSC-derived CMs responded to the L-type calcium channel blocker Diltiazem in the 
same way as hESC-derived CMs, which led to concentration-dependent reduction of up to a stop of contraction. These data are consistent with studies working with hESC-derived CMs (Xu et al. 2002). Application of the calcium channel activator Bay K8644 led to a significantly increased beating frequency. Taken together, these data reflect the normal expression of functional L-type calcium channels, one of the major components in the cardiac excitation-contraction coupling system (Bers 2002), in hESC- and hiPSC-derived CMs.

The data of this study also showed that hiPSC-derived CMs expressed sarcomeric and gap junction proteins that are critical for cardiac function, although the CMs were morphologically heterogeneous. Immunocytological staining showed a cardiac-specific protein expression in these cells, represented by cTNT, MLC2a, $\alpha$-actinin, and Cx43. These data are in line with those described in previous studies, where human pluripotent stem cells were differentiated into CMs (Kehat et al. 2001; Xu et al. 2002; Zhang et al. 2009). The antibody staining revealed an organized sarcomeric cross-striation pattern in the cells. Furthermore, $\mathrm{Cx} 43$ was expressed at cell-to-cell contacts in cardiac clusters, indicating the presence of gap junctions between CMs. Ctrl- and BrS-CMs showed no differences regarding sarcomeric organization and gap junction, indicating they are structurally comparable. This is consistent with the clinical findings that patients diagnosed with $\mathrm{BrS}$ have a structurally normal heart (Antzelevitch et al. 2005).

Electrophysiological recordings in both Ctrl- and BrS-CMs revealed on the bases of distinct classes of APs, the three major cardiac subtypes embryonic ventricular-, atrial-, and nodal-like CMs, similar to those found in hESC-derived CMs (He et al. 2003). This classification was based on the shape and properties of the APs, such as the RMP, APA, $\mathrm{V}_{\max }$, and prominence of a plateau phase. In this study, two developmental stages of CM maturation (1- and 3month-old) were analyzed electrophysiologically. The criteria for the classification due to AP parameters were selected strictly to exclude relatively immature CMs (indicated as intermediate stage). In contrast to the previous study (He et al. 2003), CMs with a RMP more positive than $-60 \mathrm{mV}$ and an APA shorter than $95 \mathrm{mV}$ were denoted as intermediate CMs and excluded from further analyses. By using these strict criteria, possible differences in $\mathrm{V}_{\max }$ among CMs caused by different developmental stages (and therefore by a different amount of sodium channel expression) were minimized for comparison of Ctrl- with BrS-CMs.

In the present study, $I_{N a}$ measurements in 1-month-old compared to 3-month-old CMs showed a significant increase of maximal $I_{N a}$ density in 3-month-old CMs, indicating a maturation 
process of the CMs over time. These findings correlate with the significantly increased $\mathrm{V}_{\max }$ in 3-month-old Ctrl-CMs compared to 1-month-old cells. In a previous study, Sartiani and colleagues investigated the maturation process of CMs obtained from hESCs within a time period of 3 months and found significant changes during in vitro differentiation (Sartiani et al. 2007). They also observed a stage-dependent significant increase of $V_{\max }$ as well as an increase of the inward L-type calcium current $\left(I_{C a L}\right)$, which is suggested to control the plateau phase of APs in hESC-derived CMs. Furthermore, the authors found that early-stage CMs (1month-old) presented a low density of inward rectifier $\left(I_{K 1}\right)$ and transient outward $\left(I_{t o l}\right)$ potassium currents, whereas late-stage CMs (3-month-old) expressed higher densities and therefore presented a larger range of APDs. Taken together with the $I_{N a}$ data of this study, it is obvious that CMs derived from human pluripotent stem cells can reach more mature phenotypes over a period of 3 months of in vitro culturing.

In this study, both 1-month-old and 3-month-old CMs were used for the identification of a BrS-specific phenotype in vitro. Due to the more mature properties in 3-month-old Ctrl- and $\mathrm{BrS}-\mathrm{CMs}$, the phenotypes of 3-month-old BrS-CMs demonstrated in the electrophyiological studies may reflect more closely the disease-specific phenotypes than 1-month-old CMs.

\subsection{BrS-CMs can recapitulate disease-specific physiological phenotypes in vitro}

It was more than 20 years ago that the Brugada brothers recognized an abnormal ECG as a distinct clinical entity, causing sudden cardiac death due to ventricular fibrillation in patients with structurally normal hearts (Brugada and Brugada, 1992), a condition which was later known as BrS. The syndrome is characterized by specific ST-segment elevation in the right precordial leads of a classical 12-lead ECG. Over the past two decades, there has been major progress in the study of genetic aspects of the syndrome as well as pathophysiology and management of patients (Wilde et al. 2002; Antzelevitch et al. 2005; Veerakul and Nademanee 2012). About $18-30 \%$ of BrS patients carry a mutation in the SCN5A gene (Antzelevitch et al. 2005) and more than 100 different SCN5A mutations are associated with BrS (Mizusawa and Wilde 2012). However, the electrophysiological mechanisms underlying the syndrome are still ambiguous and remain a matter of debate (Wilde et al. 2010). The 'repolarization hypothesis' initiated by studies in canine wedge preparations relies on transmural dispersion of repolarization between the right ventricular (outflow tract) endocardium and epicardium (Yan et al. 1998). In contrast, the 'depolarization hypothesis' supports a slowing right ventricular conduction and involvement of (mild) structural 
abnormalities (Meregalli et al. 2005). Therefore, risk stratification for sudden cardiac death, timely treatment, and prevention of sudden death, requires the crucial understanding of the pathophysiological mechanism behind the syndrome. In this study, patient-specific hiPSCs were generated from a patient with a typical BrS ECG and polymorphic ventricular tachycardia. CMs derived from these hiPSCs were used to investigate the pathophysiological mechanisms underlying the $\mathrm{BrS}$ on cellular level.

\subsubsection{Model systems for studying BrS}

In the past 5 years, an increasing number of studies were reported which use patient-specific hiPSCs for modelling cardiac channelopathies, including LQTS type 1 (Moretti et al. 2010), type 2 (Itzhaki et al. 2011; Matsa et al. 2011; Lahti et al. 2012) and type 3 (Ma et al. 2013; Terrenoire et al. 2013) as well as Timothy syndrome (Yazawa et al. 2011) and CPVT (Fatima et al. 2011; Novak et al. 2012). The present BrS-hiPSC model is the first derived from a BrS patient carrying a nonsense mutation in the $S C N 5 A$ gene. The generation of disease-specific hiPSC-CMs provides a tool for the identification of the electrophysiological and molecular mechanisms in cardiac channelopathies. This overcomes the impossibility of harvesting adult human CMs from patients and expanding them in culture.

Previously, functional characterization of sodium channels in $\mathrm{BrS}$ has been done by a number of methods. A common method is the heterologous system expressing mutated SCN5A in Xenopus oocytes, HEK293, or tsA201 cells (Bezzina et al. 1999; Barajas-Martinez et al. 2008; Petitprez et al. 2008). Heterologous expression of mutated ion channels in noncardiac cells may show disturbed function but can only predict the impact of the mutation with regard to AP generation and excitation-contraction coupling (Malan et al. 2011). Moreover, heterologous expression systems might not recapitulate the in vivo phenotype of a channelopathy because of the lack of the entire molecular complexity in which the ion channel is naturally embedded (Watanabe et al. 2011). Furthermore, transfection studies of the SCN5A mutation 5387 - 5389insTGA (p.1795insD) in HEK cells revealed kinetic properties of the cardiac sodium channel (Veldkamp et al. 2000) which could not be confirmed in adult CMs obtained from Scn5 $a^{1798 i n s D /+}$ mice (Remme et al. 2006).

A mouse model with targeted disruption of $S c n 5 a$ gene could reproduce the clinical $\mathrm{BrS}$ condition by displaying ventricular arrhythmias (Martin et al. 2010). Although mouse models may provide a more powerful tool compared to heterologous expression systems to elucidate 
the pathophysiological mechanisms underlying ion channel related cardiac diseases, they are still of limited relevance because of striking physiological differences between the mouse and human heart. In the mouse, the ventricular AP lacks a plateau phase and has a shorter APD resulting from less $I_{C a L}$ (Danik et al. 2002). This means that the spike and plateau morphology present in human, which are prerequisites for phase 2 re-entries is not present in the mouse model. In addition, establishing a mouse model of a particular mutation is laborious and expensive. A mouse model with the SCN5A gene mutation C5435A (p.S1812X) has not been established yet (Derangeon et al. 2012). Compared to all these systems, hiPSC technology provides a new platform and strategy to investigate the mechanisms of SCN5A mutations. This approach would circumvent inter-species and allogenic variations, allowing unprecedented resolution of channel behavior from patient-specific CMs.

\subsubsection{Loss-of-function of cardiac sodium channel in BrS-CMs}

The BrS-CMs carrying the SCN5A mutation C5435A (p.S1812X) demonstrated a significantly reduced (about $50 \%$ ) peak $I_{N a}$ compared to Ctrl-CMs. These data correlate with the $I_{N a}$ measurements of a BrS mouse model which was established by a knock-out of one Scn5a allele (Papadatos et al. 2002). The SCN5A mutation C5435A (p.S1812X) leads to a loss-of-function of sodium channels in CMs instead of gain-of-function, supported by persistent $I_{N a}$ measurements. No significant differences between Ctrl- and BrS-CMs could be detected, showing that the mutation does not lead to a leakage of the sodium channels. Enhanced persistent $I_{N a}$ is typical for gain-of-function of sodium channels in CMs carrying SCN5A mutations associated with LQTS (Malan et al. 2011; Ma et al. 2013). The SCN5A mutation 5387 - 5389insTGA (p.1795insD), which is located close to C5435A (p.S1812X) was further investigated using iPSC technology (Davis et al. 2012). Davis and colleagues could show in vitro that the mutation leads to both a loss- and gain-of-function of the cardiac sodium channel. The (h)iPSC derived CMs carrying this mutation demonstrated a peak $I_{N a}$ reduction (loss-of-function) on the one hand and an increased persistent $I_{N a}$ (gain-of-function) on the other hand. These disturbed sodium channel properties lead to phenotypes typical for both LQTS and BrS in affected patients and is therefore called "overlap syndrome" (Bezzina et al. 1999).

Furthermore, the activation of sodium channels in BrS-CMs of this study is delayed, represented by a right-shift of the steady-state activation curve compared to the Ctrl-CMs. Interestingly, other gating properties were not significantly influenced, such as steady-state 
inactivation, intermediate inactivation and recovery from inactivation. However, $I_{N a}$ measurements revealed a slightly but not significantly enhanced intermediate inactivation of sodium channels. An enhanced intermediate inactivation is believed to play a significant role in the pathogenesis of BrS (Wang et al. 2000). Wang and colleagues transfected mammalian cells with the SCN5A allele containing the missense mutation T1620M associated with BrS. The entry of the intermediate inactivated state measured at near physiological temperature of $32{ }^{\circ} \mathrm{C}$ was significantly enhanced in the mutant compared to the wildtype in the presence of the human $\beta_{1}$-subunit (Wang et al. 2000). However, the enhanced intermediate inactivation of the cardiac sodium channels in 3-month-old BrS-CMs in this study was not significant. The inactivation might be influenced by the temperature at which cells were measured; in this case at room temperature. It has been demonstrated that the kinetics of sodium channels are highly sensitive to temperature, showing a 2-fold faster activation and inactivation kinetics for early $I_{N a}$ at $33{ }^{\circ} \mathrm{C}$ compared to $23{ }^{\circ} \mathrm{C}$ as well as a positive shift of the activation and steady-state inactivation at the higher temperature (Nagatomo et al. 1998).

Recovery from inactivation measurements revealed that 1-month-old Ctrl-CMs recover faster than BrS-CMs. However, no significant difference was observed among 3-month-old cells, excluding a disease-specific phenotype. One explanation could be a high variability of the developmental stage among 1-month-old CMs. These data indicate that 3-month-old CMs are more suitable for $\mathrm{BrS}$ disease modelling to reveal authentic disease-specific differences.

Sodium channels play an important role in depolarization (phase 0) of the AP in the myocardial contractile cell and determine the upstroke velocity (Satin et al. 2004). The RMP is critical for the availability of sodium channels, which becomes obvious when a more positive RMP leads to more inactivated channels and finally to a reduced $\mathrm{V}_{\max }$ (Davis et al. 2012). Therefore, stringent criteria regarding RMP and APA were set in this study for the $\mathrm{V}_{\max }$ analysis. $\mathrm{V}_{\max }$ in 3-month-old BrS-CMs was significantly reduced compared to Ctrl-CMs of the same developmental stage, probably caused by the sodium channel loss-of-function in BrS-CMs. These findings were also demonstrated in hiPSC-derived CMs carrying the SCN5A mutation 5387 - 5389insTGA (p.1795insD), where a significant $I_{N a}$ reduction was observed (Davis et al. 2012). However, Davis and colleagues found that the average $V_{\max }$ in the mutated $(57.6 \mathrm{~V} / \mathrm{s})$ and control cells $(115.7 \mathrm{~V} / \mathrm{s})$ was much higher than that reported by others (Moretti et al. 2010; Itzhaki et al. 2011) or compared to the cells in the present study (see Table 12). The authors explained this differences with an alternative differentiation method they used (co-culture with END-2 cells) together with the fact that quiescent instead of 
spontaneously beating cells were used for their AP recordings. The very negative RMP of their cells $(-72.4 \mathrm{mV})$ compared to the other reports (Moretti et al. 2010; Itzhaki et al. 2011) was also discussed. A further possible explanation for the higher $\mathrm{V}_{\max }$ in cells reported by Davis and colleagues is that AP properties were recorded from cells after pacing, where artificial current is put into the cell by the patch pipette. In the present study, $V_{\max }$ was measured from spontaneous APs without pacing, and the RMP of the cells ranges from $-66.1 \mathrm{mV}$ to $-73.1 \mathrm{mV}$. These data suggest that the cells measured in the present study are at a comparable maturity stage as those used in the study by Davis and colleagues.

The authors further argue that the lack of a rapid upstroke in hiPSC-derived CMs might influence loss-of-function modelling of sodium channels (Davis et al. 2012). The $\mathrm{V}_{\max }$ data of the measured APs in this study support the idea that the higher maturation status of 3-monthold CMs with respect to $\mathrm{Na}_{\mathrm{V}} 1.5$ expression level helps to identify possible disease-specific differences between BrS- and Ctrl-CMs. For instance, whereas $\mathrm{V}_{\max }$ in 1-month-old CMs show no significant difference between BrS- and Ctrl-CMs, the difference becomes more obvious in 3-month-old cells. Here, $\mathrm{V}_{\max }$ is significantly lower in $\mathrm{BrS}$ - compared to Ctrl-CMs. These data show that it is possible to model BrS with 3-month-old hiPSC-derived CMs using the differentiation protocols described in this study, although the average $V_{\max }$ is markedly lower than reported by Davis and colleagues.

\subsubsection{Instability of AP repolarization in BrS-CMs}

Electrophysiological analyses showed three times higher arrhythmic tendencies in BrS-CMs compared to Ctrl-cells. EADs and EAD-induced triggered activity, DADs and DAD-induced triggered activity, and in the majority of cases a beat-to-beat variability of repolarization durations of sequential APs were observed. Recordings of spontaneous APs in beating BrSCMs already revealed a high beat-to-beat variability of the APDs. Since APDs are highly dependent on the beating frequency (He et al. 2003), the cells were paced at 0.8 and $1.0 \mathrm{~Hz}$ to allow comparison. The APD variability was calculated by the difference between the longest and the shortest AP within one measurement. The variability of APD50, APD70, and APD90 were significantly increased in BrS-CMs compared to Ctrl-CMs. An increased beat-to-beat variability of repolarization duration is a proarrhythmic marker (Johnson et al. 2013) and a more reliable indicator for drug-induced cardiac arrhythmias than just a prolonged APD per se (Jacobson et al. 2011). The complex mechanisms underlying this phenomenon at single-cell level are still poorly understood (Johnson et al. 2013). In their study, Johnson and colleagues 
could illustrate in canine myocytes that spontaneous $\mathrm{Ca}^{2+}$ ion release from the sarcoplasmic reticulum is a central element in triggered activity and repolarization instability (Johnson et al. 2013). By blocking ryanodine receptors with ryanodine, the authors demonstrated a significant decrease in beat-to-beat variability of APD.

To prove whether this instability of the APDs were somehow associated with the reduced $I_{N a}$ and putatively caused by the C5435A (p.S1812X) mutation in the BrS-cells of the present study, Ctrl-cells were treated with the sodium channel blocker flecainide during AP measurement. An experimentally identified concentration of $2.5 \mu \mathrm{M}$ decreased the peak $I_{N a}$ in Ctrl-CMs to 50\%, which reflected a similar level found in BrS-CMs. The Ctrl-CMs treated with flecainide during pacing showed a significantly increased beat-to-beat APD variability compared to untreated cells. These findings suggest that the decreased $I_{N a}$ might be linked to the increased APD variability. However, the reason why flecainide leads to an increased APD variability in our hiPSC-derived CMs needs to be further investigated. Future studies should also include investigation of diastolic spontaneous calcium release in BrS-CMs, as well as deciphering the roles of $\mathrm{Na}^{+} / \mathrm{Ca}^{2+}$ exchanger and L-type calcium channels in EAD- or DADinduced triggered activities in BrS-CMs.

\subsection{Molecular mechanisms underlying the loss-of-function in BrS-CMs}

The BrS patient in this study carries a point mutation in one allele of the SCN5A gene resulting in an in-frame PTC. Introducing a PTC in mRNA may lead to degradation of the mRNA template by the nonsense-mediated mRNA decay (NMD) pathway (Kuzmiak and Maquat 2006) if the PTC is less than $50-55$ nucleotides upstream of an exon-exon junction complex (Kuzmiak and Maquat 2006). Since the point mutation of the BrS patient is located on the last exon (exon 28), it is not very likely that the resulting nonsense transcripts undergo a degradation through NMD. The allele-specific expression data, which showed no difference between wildtype and mutated mRNA, endorse this assumption. However, $I_{N a}$ measurements showed a $50 \%$ reduction of the peak $I_{N a}$ in BrS-CMs compared to Ctrl-CMs. Due to the heterozygosity of the mutation, it was expected that in the BrS-CMs a truncated and a fulllength version of $\mathrm{Na}_{\vee} 1.5$ protein would be translated. Whether the truncated $\mathrm{Na}_{\vee} 1.5$ is then correctly integrated into the cell membrane (and nonfunctional due to $I_{N a}$ reduction data) or degraded directly after translation still remains an open question. 
Western blot analyses with anti-Nav1.5 antibodies binding at different epitopes of the channel were performed in this study in order to determine the expression of $\mathrm{Na}_{\mathrm{V}} 1.5$ protein. Three antibodies detecting the total $\mathrm{Na}_{\mathrm{V}} 1.5$ protein in both Ctrl- and BrS-CMs and one antibody binding at the C-terminus of the protein (amino acid residues 1978 - 2016, unable to bind the truncated protein) were tested. However, only the one recognizing the amino acid residues $1978-2016$ resulted in specific detection. The analyzed data revealed $82 \%$ of the full-length $\mathrm{Na}_{\mathrm{V}} 1.5$ protein expression in BrS-CMs compared to Ctrl-CMs. Using this antibody, the expression of the full-length $\mathrm{Na}_{\mathrm{v}} 1.5$ protein in $\mathrm{BrS}-\mathrm{CMs}$ would be expected at $50 \%$ of that in Ctrl-CMs if the expression of Nav1.5 protein was not upregulated. The data presented here indicate an upregulated expression of the wildtype $\mathrm{Na}_{\mathrm{V}} 1.5$ protein in BrS-CMs. These data are in line with the total SCN5A gene expression obtained by qPCR analyses, showing 1.6- to 2fold expression in $\mathrm{BrS}-\mathrm{CMs}$ compared to Ctrl-CMs. In parallel, allele-specific analyses showed no significant imbalance between both alleles in BrS-CMs (as discussed below). Another possibility for the upregulated protein expression is that translational readthrough of the PTC in BrS-CMs occurs (see 4.5.2). Experimental data of a previous study support this possibility (Teng et al. 2009). Teng and colleagues demonstrated translation of full-length Nav1.5 protein in HEK cells transfected only with a mutated SCN5A cDNA containing a PTC. The authors explained these findings with a lower termination efficiency of PTCs compared to natural stop codons (Teng et al. 2009). However, the question remains open, why an expression level of full-length Nav1.5 in BrS-CMs of $82 \%$ still leads to a $50 \% I_{N a}$ reduction. One reason could be that the full-length $\mathrm{Na}_{\mathrm{v}} 1.5$ obtained from translational readthrough might be non- or dysfunctional. During readthrough events, the PTC might be replaced by any amino acid (Linde and Kerem 2008), leading to an altered function of the sodium channel. Interestingly, in $S c n 5 a^{+/-}$mice, variable penetrance of the phenotype is correlated with the variable amount of functional $\mathrm{Na}_{\mathrm{v}} 1.5$ channel proteins (Leoni et al. 2010).

As mentioned above, the allele-specific SCN5A gene expression level showed no allelic imbalance between both alleles in BrS-CMs, demonstrating that both alleles are expressed equally in the differentiated BrS-CMs. The genomic DNA of the BrS-hiPSCs and hMSCs was sequenced showing that both alleles are present in the same ratio. A previous study showed a significant higher proportion of the mutated allele compared to the wildtype allele in hiPSCderived CMs (Ma et al. 2013). In that study, the point mutation G5287A (associated with LQTS type 3) leads to an amino acid exchange on protein level, where valine is substituted by methionine (p.V1763M). However, the expression level of total SCN5A was comparable 
between control and patient hiPSC-CMs. The authors assumed that more mutated and less wildtype $\mathrm{Na}_{\vee} 1.5$ was expressed. In their study, Ma and colleagues did not analyze genomic DNA of patient hiPSCs to demonstrate similar efficiencies of the individual primers for both alleles. Methodological problems need to be ruled out before the reason for allelic imbalance of SCN5A expression in CMs with the mutation G5287A but not in CMs with the C5435A mutation can be addressed.

Future studies are needed to investigate whether the mRNA template is degraded in BrS-CMs, or whether the truncated protein is integrated into the cell membrane but is nonfunctional. Furthermore, the regulation of $\mathrm{Na}_{\vee} 1.5$ protein should be studied, including CaMKIIdependent phosphorylation. Previous studies showed a stable interaction between CaMKII $\delta \mathrm{c}$ and the intracellular loop between domains 1 and 2 of $\mathrm{Na}_{\mathrm{V}} 1.5$ and CaMKII-dependent phosphorylation at multiple sites of $\mathrm{Na}_{\mathrm{V}} 1.5$ protein. This appears to evoke loss-of-function changes in $I_{N a}$ gating (Aiba et al. 2010; Hund et al. 2010; Ashpole et al. 2012).

\subsection{Possible therapeutic treatments of BrS}

To date, the only proven effective therapeutic strategy for the prevention of sudden cardiac death in BrS patients is the implantation of an ICD (Priori et al. 2013). The ICD provides an excellent and uniform efficacy for terminating ventricular fibrillation, but is not able to prevent it. Recently, clinical trials showed that catheter-based electrical epicardial substrate ablation in the right ventricular outflow tract can prevent ventricular fibrillation episodes in BrS patients (Nademanee et al. 2011). In the present discussion, both ICD implantation and catheter ablation will not be described in detail. Focus will be on the pharmacological development and treatment as well as enhancing translational readthrough of nonsense mutations instead.

\subsubsection{Potential antiarrhythmic drugs in treatment of BrS patients}

No drug therapy for BrS patients is recommended so far because clinical trials have failed to convincingly prove effectiveness (Antzelevitch et al. 2005; Márquez et al. 2005; Márquez et al. 2007; Yang et al. 2009). However, two drugs (isoproterenol and quinidine) have been reported to prevent the recurrence of ventricular fibrillation and repetitive traumatizing therapeutic shocks in patients with an ICD during electrical storm (Maury et al. 2004; Mok et al. 2004; Bettiol et al. 2005). Currently, quinidine is reconsidered for the treatment of $\mathrm{BrS}$ (Yang et al. 2009). Drug therapy may play a complementary role to the ICD by reducing the 
number of ICD shocks delivered. Prevention of ventricular fibrillation also contributes to the improvement in the quality of life of the patients by avoiding uncomfortable ICD shock deliveries.

According to the repolarization hypothesis, an outward shift in the balance of transmembrane ionic currents at the end of phase 1 and phase 2 of the AP leads to the loss of the phase 2 AP plateau. This cellular mechanism is believed to cause a marked transmural voltage gradient that leads to the ST segment elevation and the higher susceptibility for ventricular fibrillation in BrS (Antzelevitch 2001). At the end of phase 1 and phase 2 of the AP, the outward current is mainly due to activation of the transient outward potassium current $\left(I_{t o}\right)$ and the inward current is mainly due to activation of an inward calcium current $\left(I_{C a}\right)$ and an inward $I_{N a}$. Due to the loss-of-function of sodium channels in $\mathrm{BrS}$, the net outward shift of the current balance leads to a loss of plateau or phase 2 of the AP (Antzelevitch 2001). In theory, this means that drugs that counteract the ionic current imbalance in BrS could be used for treatment.

Quinidine is a class I antiarrhythmic agent, which blocks the calcium-independent $I_{t o}$ and $I_{k r}$ (Yan and Antzelevitch 1999; Priori et al. 2013). Previous studies showed that in animal models, quinidine $(5 \mu \mathrm{M})$ inhibited $I_{t o}$ and exerted an antiarrhythmic effect by restoring the AP plateau in the epicardium (Yan and Antzelevitch 1999). In the present study, although the treatment of BrS-CMs with quinidine $(5 \mu \mathrm{M})$ resulted in reduced beating frequencies and prolonged APDs and restored the AP plateau, it did not reduce the beat-to-beat variability of repolarization duration and EADs. These data indicate that quinidine cannot completely restore the normal properties of the AP. Clinical trials with quinidine were already performed to treat $\mathrm{BrS}$ patients. It could be shown that quinidine suppresses spontaneous ventricular tachyarrhythmias and prevented ventricular fibrillation induction in 22 of the 25 (symptomatic and asymptomatic) BrS patients (Belhassen et al. 2004). However, the basis for quinidine efficacy remains to be elucidated due to a limited number of patients in this study and a high incidence of side effects (36\%). Furthermore, although quinidine is believed to be the only effective oral medication to prevent ventricular arrhythmias and fibrillation in BrS patients, it is inaccessible in many countries (Viskin et al. 2013). Further studies with regards to the effect of quinidine on $I_{t o}, I_{C a}$ and $I_{N a}$ in BrS-CMs should be performed. In addition, other $I_{t o}$ blockers without strong sodium channel effects, for example, tedisamil, which may be more effective than quinidine (Antzelevitch 2001), should be tested in BrS-CMs. 
Another group of drugs that can counteract the ionic current imbalance in $\mathrm{BrS}$ are the drugs which can result in a marked increase in $I_{C a}$, and compensate for the prominent loss of plateau during phase 2 of the AP. By this way, they may result in a decrease in electrical heterogeneity underlying the ST elevation in BrS patients. For example, isoproterenol, which induces the $I_{C a L}$, has been proven to be useful for the treatment of electrical storm in $\mathrm{BrS}$ (Maury et al. 2004). However, convincing data on its therapeutic mechanism of action is not available (Priori et al. 2013). The effect of isoproterenol on BrS-CMs needs to be studied next. The most obvious difference between BrS- and Ctrl-CMs is an $I_{N a}$ reduction of about $50 \%$ in BrS-CMs, which could imply that an effective treatment of the BrS patient would be to induce the enhancement of the cardiac $I_{N a}$.

\subsubsection{Readthrough therapy for suppression of nonsense mutation}

In the past two decades, remarkable advances in the ability to treat genetic disorders have been made, including the search for mutation-targeted molecular therapies. One of such therapies is called nonsense suppression therapy, using compounds or small-interfering RNAs (siRNAs) to induce the translation machinery to recode a nonsense codon into a sense codon. Thereby a translational readthrough of the PTC is promoted to enable the synthesis of a fulllength functional protein (Keeling and Bedwell 2011), given that enough nonsense mRNA transcripts are not degraded by the NMD pathway.

Different approaches might lead to PTC readthrough, such as siRNA targeting translationtermination factors, which would otherwise cause the release of the shortened polypeptide from the ribosome due to the PTC. Another therapeutic approach is focusing on aminoglycoside antibiotics, such as G418 or gentamicin (reviewed by Linde and Kerem 2008). Aminoglycosides can bind to the A site of the ribosomal RNA translational complex and thereby cause a conformational change. This in turn leads to a reduced accuracy between codon and anticodon pairing, which enables a readthrough of the PTC. Aminoglycosides have shown minimal effects on the normal termination sites (Linde and Kerem 2008). The authors argue that in eukaryotes, the efficiency of normal translation termination is enhanced by certain upstream and downstream sequences as well as the proximity of the natural stop codon to the poly(A) tail, which is usually not the case in PTCs.

Aminoglycosides-induced nonsense mutation readthrough and thus synthesis of functional full-length protein was first reported in nonsense mutations resulting in cystic fibrosis (Howard et al. 1996) and Duchenne muscular dystrophy (Howard et al. 2000). In 2009, Teng 
and colleagues were the first who tried to restore the function of mutated Nav1.5 channels caused by the PTC mutation W822X using the readthrough strategy (Teng et al. 2009). They transfected HEK293 cells with mutated and nonmutated SCN5A cDNA. HEK293 cells transfected only with the mutated channel showed an $I_{N a}$ density of $<3 \%$ compared to HEK293 cells with the wildtype channel and reached $30 \%$ of the wildtype level after treatment with gentamicin or G418. Cells transfected with both cDNAs showed an $I_{N a}$ density of $56 \%$ compared to the wildtype level. These cells increased their peak $I_{N a}$ up to $70 \%$ of the wildtype level when treated with gentamicin or G418. Western blot analyses confirmed a higher expression of the full-length protein after antibiotic treatment. In addition, the authors showed that siRNAs targeting the eukaryotic release factor eRF3a revealed similar results as antibiotic treatment regarding protein expression and $I_{N a}$ measurements. Although the expression of full-length $\mathrm{Na}_{\mathrm{V}} 1.5$ protein could be partially restored in that study, the following impact on the electrical behavior in CMs could not be tested. The hiPSC technology would provide the ideal model system to investigate possible effects of the readthrough treatment in human CMs. A potential impact on the $I_{N a}$ density in BrS-CMs could be subsequently measured after treatment. In addition, AP recordings can directly show if an enhanced $I_{N a}$ would have positive effects on the AP morphology and properties as well as the rhythmic beating of the cell. Hence, suppression experiments of the SCN5A nonsense mutation C5435A (p.S1812X) in the generated BrS-CMs of this study could shed light on the relation between partial restoration of the sodium channel by translational readthrough, and the accompanying reversion of electrophyiological behavior of BrS-CMs.

Although aminoglycosides like gentamicin already demonstrated promising results in many studies, the benefit of long-term use is limited because of the antibiotic related severe side effects, such as kidney damage (Mingeot-Leclercq and Tulkens 1999). However, highthroughput screens identified the small molecule PTC124 without antibiotic-associated side effects, which can promote ribosomal readthrough of PTCs (Hirawat et al. 2007; Welch et al. 2007). Importantly, PTC124 does not affect the termination at natural stop codons at the end of a coding sequence (Hirawat et al. 2007). First clinical trials showed improved electrophysiological function in patients with cystic fibrosis caused by PTCs in the cystic fibrosis transmembrane conductance regulator mRNA after treatment with PTC124 (Kerem et al. 2008). In addition, an increased full-length synthesis of the cystic fibrosis transmembrane conductance regulator protein was evoked by PTC124. However, these studies revealed a high variability in the response to the readthrough treatments, possibly due to different readthrough efficiencies at the various triplet codons (reviewed by Linde and Kerem 2008). 
Readthrough efficiency is highest on a UGA codon and lowest on a UAA codon (UGA > UAG > UAA; Howard et al. 2000). Additionally, it has been demonstrated that the base following directly the PTC might also play an important role for the readthrough efficiency. The efficacies regarding chemical composition of the aminoglycosides, the brand, and the origin of production have also been discussed (Linde and Kerem 2008).

Taken together, PTC124 might also provide a possible approach to treat BrS caused by the nonsense mutation C5435A (p.S1812X), since the mRNA is not believed to be degraded through NMD (as discussed in 4.4). Furthermore, BrS-CMs can be used in high throughput screening of drugs or small molecules that can induce translational readthrough of this mutation. This might contribute to personalized genetic medicine that aims to treat patients according to their specific genetic defects and molecular phenotypes.

\subsection{Conclusion and future perspectives}

In conclusion, the findings of this study suggest that patient-specific hiPSCs can serve as a suitable model for studying sodium channel mutations. CMs derived from BrS-hiPSCs display features of loss-of-function of sodium channels and corresponding changes in AP recordings such as reduced upstroke velocity and increased beat-to-beat variability of repolarization duration. Although the phenotypic immaturity of hiPSC-derived CMs has been discussed for their feasibility in studying adult channelopathies, the data presented in this study demonstrate that these cells can recapitulate human sodium channelopathy in a dish. The data also show that hiPSC-derived CMs can provide a platform for investigating the effect of antiarrhythmic drugs and studying their underlying physiological mechanisms.

In the future, it is of paramount importance to perform expression profiling between family members, which may help to identify critical genetic modifiers of disease severity. The underlying molecular mechanisms of loss-of-function of sodium channels need to be further investigated. Another potential area of using the BrS-CMs will be in high throughput screening of drugs or small molecules that can enhance sodium channel activity, or induce translational readthrough of nonsense mutations. In addition, hiPSC-derived CMs may move us closer to personalized medicine for $\mathrm{BrS}$, where drug regimens can be tested in vitro before being administered to the patient. 


\section{Summary}

Brugada syndrome $(\mathrm{BrS})$, a cardiac genetic disease, is one of the major causes of sudden cardiac death in healthy young people. However, the underlying electrophysiological and molecular mechanisms have not been completely understood. A major challenge in cardiac translational research is the lack of tissue culture systems replicating human pathology to study disease mechanisms and to identify druggable targets. Aim of this study was to study the pathophysiological and molecular mechanisms of $\mathrm{BrS}$ in vitro using patient-specific induced pluripotent stem cells (iPSCs) as a renewable and unlimited source for cardiomyocytes (CMs).

In this study, human iPSCs were generated from a 45-year-old healthy donor and a 50-yearold patient with BrS putatively caused by the heterozygous point mutation C5435A in the gene SCN5A coding for the $\alpha$-subunit of the cardiac sodium channel ( $\left.\mathrm{Na}_{\mathrm{V}} 1.5\right)$. The generated human iPSCs showed pluripotency and were able to differentiate into spontaneously beating CMs. Sodium current $\left(I_{N a}\right)$ measurements revealed a significantly reduced current with a delayed activation in BrS-CMs compared to control cells, indicating a Nav1.5 loss of function. In BrS-CMs, the intermediate inactivation of sodium channels was slightly but not significantly enhanced whereas steady-state inactivation, recovery from inactivation, and persistent $I_{N a}$ were not affected. In addition, an electrophysiological maturation process could also be detected, which was shown by a larger $I_{N a}$ in 3-month-old compared to 1-month-old CMs.

Action potential (AP) measurements showed a reduced $\mathrm{V}_{\max }$ and higher arrhythmic tendencies in BrS-CMs compared to control cells. A significantly higher variability of the AP durations as well as early and delayed afterdepolarizations could be observed. AP measurements in control cells under the treatment with the sodium channel blocker flecainide suggest that the increased AP duration variability in $\mathrm{BrS}-\mathrm{CMs}$ is linked to the reduced $I_{N a}$. However, treatment of BrS-CMs with the class I antiarrhythmic agent quinidine, which is currently investigated for its therapeutic effect on BrS, could not reduce the beat-to-beat variability of AP durations.

Furthermore, quantitative real-time PCR showed that SCN5A was slightly but not significantly upregulated in BrS-CMs compared to the control cells with an allele-specific balanced expression. However, full-length $\mathrm{Na}_{\mathrm{V}} 1.5$ protein was detected in $\mathrm{BrS}-\mathrm{CMs}$ at a level of $82 \%$ compared to the control. The question whether the truncated Nav1.5 protein is integrated into the cell membrane or regulated by increased protein degradation remains open. 
The data of this study demonstrate that patient-specific hiPSCs can be used to model $\mathrm{BrS}$ and provide a platform for the development of personalized drug therapy. 


\section{References}

Aasen T, Raya A, Barrero MJ, Garreta E, Consiglio A, Gonzalez F, Vassena R, Bilić J, Pekarik V, Tiscornia G, Edel M, Boué S, Izpisúa Belmonte JC (2008). Efficient and rapid generation of induced pluripotent stem cells from human keratinocytes. Nat Biotechnol 26: $1276-1284$

Abriel H (2010). Cardiac sodium channel $\mathrm{Na}_{\mathrm{V}} 1.5$ and interacting proteins: Physiology and pathophysiology. J Mol Cell Cardiol 48: 2-11

Aiba T, Hesketh GG, Liu T, Carlisle R, Villa-Abrille MC, O'Rourke B, Akar FG, Tomaselli GF (2010). $\mathrm{Na}^{+}$channel regulation by $\mathrm{Ca}^{2+} /$ calmodulin and $\mathrm{Ca}^{2+} /$ calmodulindependent protein kinase II in guinea-pig ventricular myocytes. Cardiovasc Res 85: 454-463

Antzelevitch C (2001). The Brugada syndrome: ionic basis and arrhythmia mechanisms. J Cardiovasc Electrophysiol 12: 268-72

Antzelevitch C, Brugada P, Borggrefe M, Brugada J, Brugada R, Corrado D, Gussak I, LeMarec H, Nademanee K, Perez Riera AR, Shimizu W, Schulze-Bahr E, Tan H, Wilde A (2005). Brugada syndrome: report of the second consensus conference: endorsed by the Heart Rhythm Society and the European Heart Rhythm Association. Circulation 111: 659-670

Antzelevitch C, Fish JM (2006). Therapy for the Brugada syndrome. Handb Exp Pharmacol 171: $305-330$

Aoi T, Yae K, Nakagawa M, Ichisaka T, Okita K, Takahashi K, Chiba T, Yamanaka S (2008). Generation of pluripotent stem cells from adult mouse liver and stomach cells. Science 321: 699-702

Ashpole NM, Herren AW, Ginsburg KS, Brogan JD, Johnson DE, Cummins TR, Bers DM, Hudmon A (2012). Ca2+/calmodulin-dependent protein kinase II (CaMKII) regulates cardiac sodium channel Nav1.5 gating by multiple phosphorylation sites. J Biol Chem 287: 19856-19869

Baker DE, Harrison NJ, Maltby E, Smith K, Moore HD, Shaw PJ, Heath PR, Holden H, Andrews PW (2007). Adaptation to culture of human embryonic stem cells and oncogenesis in vivo. Nat Biotechnol 25: 207-215

Bankston JR, Yue M, Chung W, Spyres M, Pass RH, Silver E, Sampson KJ, Kass RS (2007). A novel and lethal de novo LQT-3 mutation in a newborn with distinct molecular pharmacology and therapeutic response. PLoS One 2: e1258

Barajas-Martínez HM, Hu D, Cordeiro JM, Wu Y, Kovacs RJ, Meltser H, Kui H, Elena

B, Brugada R, Antzelevitch C, Dumaine R (2008). Lidocaine-induced Brugada syndrome phenotype linked to a novel double mutation in the cardiac sodium channel. Circ Res 103: 396-404 
Belhassen B, Glick A, Viskin S (2004). Efficacy of quinidine in high-risk patients with Brugada syndrome. Circulation 110: 1731-1737

Bellin M, Marchetto MC, Gage FH, Mummery CL (2012). Induced pluripotent stem cells: the new patient? Nat Rev Mol Cell Biol 13: 713-726

Bennett PB, Yazawa K, Makita N, George AL Jr (1995). Molecular mechanism for an inherited cardiac arrhythmia. Nature 376: 683-685

Bers DM (2002). Cardiac excitation-contraction coupling. Nature 415: 198-205

Bettiol K, Gianfranchi L, Scarfò S, Pacchioni F, Pedaci M, Alboni P (2005). Successful treatment of electrical storm with oral quinidine in Brugada syndrome. Ital Heart J 6: 601602

Bezzina C, Veldkamp MW, van Den Berg MP, Postma AV, Rook MB, Viersma JW, van Langen IM, Tan-Sindhunata G, Bink-Boelkens MT, van Der Hout AH, Mannens MM, Wilde AA (1999). A single $\mathrm{Na}(+)$ channel mutation causing both long-QT and Brugada syndromes. Circ Res 85: 1206-1213

Bhattacharya B, Miura T, Brandenberger R, Mejido J, Luo Y, Yang AX, Joshi BH, Thies RS, Amit M, Lyons I, Condie BG, Itskovitz-Eldor J, Rao MS, Puri RK (2004). Gene expression in human embryonic stem cell lines: unique molecular signature. Blood 103: 2956-2964

Blechschmidt S, Haufe V, Benndorf K, Zimmer T (2008). Voltage-gated $\mathrm{Na}^{+}$channel transcript patterns in the mammalian heart are species-dependent. Prog Biophys Mol Biol 98: 309-318

Blum B, Benvenisty N (2009). The tumorigenicity of diploid and aneuploid human pluripotent stem cells. Cell Cycle 8: 3822-3830

Boyer LA, Lee TI, Cole MF, Johnstone SE, Levine SS, Zucker JP, Guenther MG, Kumar RM, Murray HL, Jenner RG, Gifford DK, Melton DA, Jaenisch R, Young RA (2005). Core transcriptional regulatory circuitry in human embryonic stem cells. Cell. 122: 947-956

Briggs R, King TJ (1952). Transplantation of living nuclei from blastula cells into enucleated frogs' eggs. Proc Natl Acad Sci U S A 38: 455-463

Brugada P, Brugada J (1992). Right bundle branch block, persistent ST segment elevation and sudden cardiac death: a distinct clinical and electrocardiographic syndrome. A multicenter report. J Am Coll Cardiol 20:1391-1396

Brugada P, Brugada R, Brugada J, Geelen P (1999). Use of the prophylactic implantable cardioverter defibrillator for patients with normal hearts. Am J Cardiol 83: 98D-100D 
Catterall WA, Goldin AL, Waxman SG (2005). International Union of Pharmacology. XLVII. Nomenclature and structure-function relationships of voltage-gated sodium channels Pharmacol Rev 57: 397-409

Chambers I, Colby D, Robertson M, Nichols J, Lee S, Tweedie S, Smith A (2003). Functional expression cloning of Nanog, a pluripotency sustaining factor in embryonic stem cells. Cell 113: 643-655

Chen IP, Fukuda K, Fusaki N, Iida A, Hasegawa M, Lichtler A, Reichenberger EJ (2013). Induced pluripotent stem cell reprogramming by integration-free Sendai virus vectors from peripheral blood of patients with craniometaphyseal dysplasia. Cell Reprogram 15: $503-513$

Chen JZ, Xie XD, Wang XX, Tao M, Shang YP, Guo XG (2004). Single nucleotide polymorphisms of the $S C N 5 A$ gene in Han Chinese and their relation with Brugada syndrome. Chin Med J (Engl.) 117: 652-656

Chen PS, Priori SG (2008). The Brugada syndrome. J Am Coll Cardiol 51: 1176-1180

Chen Q, Kirsch GE, Zhang D, Brugada R, Brugada J, Brugada P, Potenza D, Moya A, Borggrefe M, Breithardt G, Ortiz-Lopez R, Wang Z, Antzelevitch C, O'Brien RE, Schulze-Bahr E, Keating MT, Towbin JA, Wang Q (1998). Genetic basis and molecular mechanism for idiopathic ventricular fibrillation. Nature 392: 293-296

Chivukula RR, Mendell JT (2009). Abate and switch: miR-145 in stem cell differentiation. Cell 137: 606-608

Clark AT, Rodriguez RT, Bodnar MS, Abeyta MJ, Cedars MI, Turek PJ, Firpo MT, Reijo Pera RA (2004). Human STELLAR, NANOG and GDF3 genes are expressed in pluripotent cells and map to chromosome 12p13, a hotspot for teratocarcinoma. Stem Cells 22: $169-179$

Danik S, Cabo C, Chiello C, Kang S, Wit AL, Coromilas J (2002). Correlation of repolarization of ventricular monophasic action potential with ECG in the murine heart. Am J Physiol Heart Circ Physiol 283: H372-381

Davis RL, Weintraub H, Lassar AB (1987). Expression of a single transfected cDNA converts fibroblasts to myoblasts. Cell 51: 987-1000

Davis RP, Casini S, van den Berg CW, Hoekstra M, Remme CA, Dambrot C, Salvatori D, Oostwaard DW, Wilde AA, Bezzina CR, Verkerk AO, Freund C, Mummery CL (2012). Cardiomyocytes derived from pluripotent stem cells recapitulate electrophysiological characteristics of an overlap syndrome of cardiac sodium channel disease. Circulation 125: 3079-3091

Derangeon M, Montnach J, Baró I, Charpentier F (2012). Mouse Models of SCN5ARelated Cardiac Arrhythmias. Front Physiol 3: 210 
Dick E, Rajamohan D, Ronksley J, Denning C (2010). Evaluating the utility of cardiomyocytes from human pluripotent stem cells for drug screening. Biochem Soc Trans 38: $1037-1045$

Draper JS, Smith K, Gokhale P, Moore HD, Maltby E, Johnson J, Meisner L, Zwaka TP, Thomson JA, Andrews PW (2004). Recurrent gain of chromosomes 17q and 12 in cultured human embryonic stem cells. Nat Biotechnol 22: 53-54

Durruthy-Durruthy J, Briggs SF, Awe J, Ramathal CY, Karumbayaram S, Lee PC, Heidmann JD, Clark A, Karakikes I, Loh KM, Wu JC, Hoffman AR, Byrne J, Reijo Pera RA, Sebastiano V (2014). Rapid and Efficient Conversion of Integration-Free Human Induced Pluripotent Stem Cells to GMP-Grade Culture Conditions. PLoS One 9: e94231

Enver T, Soneji S, Joshi C, Brown J, Iborra F, Orntoft T, Thykjaer T, Maltby E, Smith K, Abu Dawud R, Jones M, Matin M, Gokhale P, Draper J, Andrews PW (2005). Cellular differentiation hierarchies in normal and culture-adapted human embryonic stem cells. Hum Mol Genet 14: 3129-3140

Evans MJ, Kaufman MH (1981). Establishment in culture of pluripotential cells from mouse embryos. Nature 292: 154-156

Extramiana F, Seitz J, Maison-Blanche P, Badilini F, Haggui A, Takatsuki S, Milliez P, Denjoy I, Cauchemez B, Beaufils P, Leenhardt A (2006). Quantitative assessment of ST segment elevation in Brugada patients. Heart Rhythm 3: 1175-1181

Fatima A, Xu G, Shao K, Papadopoulos S, Lehmann M, Arnáiz-Cot JJ, Rosa AO, Nguemo F, Matzkies M, Dittmann S, Stone SL, Linke M, Zechner U, Beyer V, Hennies HC, Rosenkranz S, Klauke B, Parwani AS, Haverkamp W, Pfitzer G, Farr M, Cleemann L, Morad M, Milting H, Hescheler J, Saric T (2011). In vitro modeling of ryanodine receptor 2 dysfunction using human induced pluripotent stem cells. Cell Physiol Biochem 28: 579-592

Fusaki N, Ban H, Nishiyama A, Saeki K, Hasegawa M (2009). Efficient induction of transgene-free human pluripotent stem cells using a vector based on Sendai virus, an RNA virus that does not integrate into the host genome. Proc Jpn Acad Ser B Phys Biol Sci 85: $348-362$

Grisham J (2000). Pigs cloned for first time. Nat Biotechnol 18: 365

Groeneveld GJ, Veldink JH, van der Tweel I, Kalmijn S, Beijer C, de Visser M, Wokke JH, Franssen H, van den Berg LH (2003). A randomized sequential trial of creatine in amyotrophic lateral sclerosis. Ann Neurol 53: 437-445

Gunaseeli I, Doss MX, Antzelevitch C, Hescheler J, Sachinidis A (2010). Induced pluripotent stem cells as a model for accelerated patient- and disease-specific drug discovery. Curr Med Chem 17: 759-766 
Gurdon, JB (1962). The developmental capacity of nuclei taken from intestinal epithelium cells of feeding tadpoles. J Embryol Exp Morphol 10: 622-640

Haase A, Olmer R, Schwanke K, Wunderlich S, Merkert S, Hess C, Zweigerdt R, Gruh I, Meyer J, Wagner S, Maier LS, Han DW, Glage S, Miller K, Fischer P, Schöler HR, Martin U (2009). Generation of induced pluripotent stem cells from human cord blood. Cell Stem Cell 5: 434-441

Hanna J, Wernig M, Markoulaki S, Sun CW, Meissner A, Cassady JP, Beard C, Brambrink T, Wu LC, Townes TM, Jaenisch R (2007). Treatment of sickle cell anemia mouse model with iPS cells generated from autologous skin. Science 318: 1920-1923

He JQ, Ma Y, Lee Y, Thomson JA, Kamp TJ (2003). Human embryonic stem cells develop into multiple types of cardiac myocytes: action potential characterization. Circ Res 93: 3239

Henderson JK, Draper JS, Baillie HS, Fishel S, Thomson JA, Moore H, Andrews PW (2002). Preimplantation human embryos and embryonic stem cells show comparable expression of stage-specific embryonic antigens. Stem Cells 20: 329-337

Hirawat S, Welch EM, Elfring GL, Northcutt VJ, Paushkin S, Hwang S, Leonard EM, Almstead NG, Ju W, Peltz SW, Miller LL (2007). Safety, tolerability, and pharmacokinetics of PTC124, a nonaminoglycoside nonsense mutation suppressor, following single- and multiple-dose administration to healthy male and female adult volunteers. J Clin Pharmacol 47: 430-444

Hochedlinger K, Jaenisch $\mathbf{R}$ (2002). Monoclonal mice generated by nuclear transfer from mature B and T donor cells. Nature 415: 1035-1038

Hockemeyer D1, Wang H, Kiani S, Lai CS, Gao Q, Cassady JP, Cost GJ, Zhang L, Santiago Y, Miller JC, Zeitler B, Cherone JM, Meng X, Hinkley SJ, Rebar EJ, Gregory PD, Urnov FD, Jaenisch R (2011). Genetic engineering of human pluripotent cells using TALE nucleases. Nat Biotechnol 29: 731-734

Howard M, Frizzell RA, Bedwell DM (1996). Aminoglycoside antibiotics restore CFTR function by overcoming premature stop mutations. Nat Med 2: 467-469

Howard MT, Shirts BH, Petros LM, Flanigan KM, Gesteland RF, Atkins JF (2000). Sequence specificity of aminoglycoside-induced stop condon readthrough: potential implications for treatment of Duchenne muscular dystrophy. Ann Neurol 48: 164-169

Huang P, He Z, Ji S, Sun H, Xiang D, Liu C, Hu Y, Wang X, Hui L (2011). Induction of functional hepatocyte-like cells from mouse fibroblasts by defined factors. Nature 475: 386389

Hund TJ, Koval OM, Li J, Wright PJ, Qian L, Snyder JS, Gudmundsson H, Kline CF, Davidson NP, Cardona N, Rasband MN, Anderson ME, Mohler PJ (2010). A $\beta(\mathrm{IV})$ - 
spectrin/CaMKII signaling complex is essential for membrane excitability in mice. $J$ Clin Invest 120: 3508-3519

Hyslop L, Stojkovic M, Armstrong L, Walter T, Stojkovic P, Przyborski S, Herbert M, Murdoch A, Strachan T, Lako M (2005). Downregulation of NANOG induces differentiation of human embryonic stem cells to extraembryonic lineages. Stem Cells 23: 1035-1043

Ibrahimi A, Vande Velde G, Reumers V, Toelen J, Thiry I, Vandeputte C, Vets S, Deroose C, Bormans G, Baekelandt V, Debyser Z, Gijsbers R (2009). Highly efficient multicistronic lentiviral vectors with peptide 2A sequences. Hum Gene Ther 20: 845-860

Ieda M, Fu JD, Delgado-Olguin P, Vedantham V, Hayashi Y, Bruneau BG, Srivastava D (2010). Direct reprogramming of fibroblasts into functional cardiomyocytes by defined factors. Cell 142:375-386

International Stem Cell Initiative (2007). Characterization of human embryonic stem cell lines by the International Stem Cell Initiative. Nat Biotechnol 25: 803-816

Inzunza J, Sahlén S, Holmberg K, Strömberg AM, Teerijoki H, Blennow E, Hovatta O, Malmgren H (2004). Comparative genomic hybridization and karyotyping of human embryonic stem cells reveals the occurrence of an isodicentric X chromosome after longterm cultivation. Mol Hum Reprod 10: 461-466

Itskovitz-Eldor J, Schuldiner M, Karsenti D, Eden A, Yanuka O, Amit M, Soreq H, Benvenisty N (2000). Differentiation of human embryonic stem cells into embryoid bodies compromising the three embryonic germ layers. Mol Med 6: 88-95

Itzhaki I, Maizels L, Huber I, Zwi-Dantsis L, Caspi O, Winterstern A, Feldman O, Gepstein A, Arbel G, Hammerman H, Boulos M, Gepstein L (2011). Modelling the long QT syndrome with induced pluripotent stem cells. Nature 471: 225-229

Iwasa H, Itoh T, Nagai R, Nakamura Y, Tanaka T (2000). Twenty single nucleotide polymorphisms (SNPs) and their allelic frequencies in four genes that are responsible for familial long QT syndrome in the Japanese population. J Hum Genet 45: 182-183

Jacobson I, Carlsson L, Duker G (2011). Beat-by-beat QT interval variability, but not QT prolongation per se, predicts drug-induced torsades de pointes in the anaesthetised methoxamine-sensitized rabbit. J Pharmacol Toxicol Methods 63: 40-46

Johnson DM, Heijman J, Bode EF, Greensmith DJ, van der Linde H, Abi-Gerges N, Eisner DA, Trafford AW, Volders PG (2013). Diastolic spontaneous calcium release from the sarcoplasmic reticulum increases beat-to-beat variability of repolarization in canine ventricular myocytes after $\beta$-adrenergic stimulation. Circ Res 112: 246-256

Keeling KM, Bedwell DM (2011). Suppression of nonsense mutations as a therapeutic approach to treat genetic diseases. Wiley Interdiscip Rev RNA 2: 837-852 
Kehat I, Kenyagin-Karsenti D, Snir M, Segev H, Amit M, Gepstein A, Livne E, Binah O, Itskovitz-Eldor J, Gepstein L (2001). Human embryonic stem cells can differentiate into myocytes with structural and functional properties of cardiomyocytes. J Clin Invest 108: 407-414

Kelly SJ (1977). Studies of the developmental potential of 4- and 8-cell stage mouse blastomeres. J Exp Zool 200: 365-376

Kerem E, Hirawat S, Armoni S, Yaakov Y, Shoseyov D, Cohen M, Nissim-Rafinia M, Blau H, Rivlin J, Aviram M, Elfring GL, Northcutt VJ, Miller LL, Kerem B, Wilschanski M (2008). Effectiveness of PTC124 treatment of cystic fibrosis caused by nonsense mutations: a prospective phase II trial. Lancet 372: 719-727

Kim D, Kim CH, Moon JI, Chung YG, Chang MY, Han BS, Ko S, Yang E, Cha KY, Lanza R, Kim KS (2009). Generation of human induced pluripotent stem cells by direct delivery of reprogramming proteins. Cell Stem Cell 4: 472-476

Kim J, Chu J, Shen X, Wang J, Orkin SH (2008). An extended transcriptional network for pluripotency of embryonic stem cells. Cell 132: 1049-1061

Kim JB, Sebastiano V, Wu G, Araúzo-Bravo MJ, Sasse P, Gentile L, Ko K, Ruau D, Ehrich M, van den Boom D, Meyer J, Hübner K, Bernemann C, Ortmeier C, Zenke M, Fleischmann BK, Zaehres H, Schöler HR (2009). Oct4-induced pluripotency in adult neural stem cells. Cell 136: 411-419

Kuzmiak HA, Maquat LE (2006). Applying nonsense-mediated mRNA decay research to the clinic: progress and challenges. Trends Mol Med 12: 306-316

Lahti AL, Kujala VJ, Chapman H, Koivisto AP, Pekkanen-Mattila M, Kerkelä E, Hyttinen J, Kontula K, Swan H, Conklin BR, Yamanaka S, Silvennoinen O, AaltoSetälä K (2012). Model for long QT syndrome type 2 using human iPS cells demonstrates arrhythmogenic characteristics in cell culture. Dis Model Mech 5: 220-230

Laustriat D, Gide J, Peschanski M (2010). Human pluripotent stem cells in drug discovery and predictive toxicology. Biochem Soc Trans 38: 1051-1057

Leoni AL1, Gavillet B, Rougier JS, Marionneau C, Probst V, Le Scouarnec S, Schott JJ, Demolombe S, Bruneval P, Huang CL, Colledge WH, Grace AA, Le Marec H, Wilde AA, Mohler PJ, Escande D, Abriel H, Charpentier F (2010). Variable Na(v)1.5 protein expression from the wild-type allele correlates with the penetrance of cardiac conduction disease in the Scn5a(+/-) mouse model. PLoS One 5: e9298

Levine AJ, Brivanlou AH (2005). GDF3, a BMP inhibitor, regulates cell fate in stem cells and early embryos. Development 133: 209-216

Lian X, Hsiao C, Wilson G, Zhu K, Hazeltine LB, Azarin SM, Raval KK, Zhang J, Kamp TJ, Palecek SP (2012). Robust cardiomyocyte differentiation from human 
pluripotent stem cells via temporal modulation of canonical Wnt signaling. Proc Natl Acad Sci U S A 109: 1848-1857

Lian X, Zhang J, Azarin SM, Zhu K, Hazeltine LB, Bao X, Hsiao C, Kamp TJ, Palecek SP (2013). Directed cardiomyocyte differentiation from human pluripotent stem cells by modulating Wnt/ $\beta$-catenin signaling under fully defined conditions. Nat Protoc 8: 162-175

Linde L, Kerem B (2008). Introducing sense into nonsense in treatments of human genetic diseases. Trends Genet 24: 552-563

Ma D, Wei H, Zhao Y, Lu J, Li G, Sahib NB, Tan TH, Wong KY, Shim W, Wong P, Cook SA, Liew R (2013). Modeling type 3 long QT syndrome with cardiomyocytes derived from patient-specific induced pluripotent stem cells. Int J Cardiol 168: 5277-5286

Ma J, Guo L, Fiene SJ, Anson BD, Thomson JA, Kamp TJ, Kolaja KL, Swanson BJ, January CT (2011). High purity human-induced pluripotent stem cell-derived cardiomyocytes: electrophysiological properties of action potentials and ionic currents. Am J Physiol Heart Circ Physiol 301: 2006-2017

Maherali M, Sridharan R, Xie W, Utikal J, Eminli S, Arnold K, Stadtfeld M, Yachechko R, Tchieu J, Jaenisch R, Plath K, Hochedlinger K (2007). Directly reprogrammed fibroblasts show global epigenetic remodeling and widespread tissue contribution. Cell Stem Cell 1: 55-70

Maier SK, Westenbroek RE, Schenkman KA, Feigl EO, Scheuer T, Catterall WA (2002). An unexpected role for brain-type sodium channels in coupling of cell surface depolarization to contraction in the heart. Proc Natl Acad Sci U S A 99: 4073-4078

Maier SK, Westenbroek RE, McCormick KA, Curtis R, Scheuer T, Catterall WA (2004). Distinct subcellular localization of different sodium channel alpha and beta subunits in single ventricular myocytes from mouse heart. Circulation 109: 1421-1427

Makita N, Behr E, Shimizu W, Horie M, Sunami A, Crotti L, Schulze-Bahr E, Fukuhara S, Mochizuki N, Makiyama T, Itoh H, Christiansen M, McKeown P, Miyamoto K, Kamakura S, Tsutsui H, Schwartz PJ, George AL Jr, Roden DM (2008). The E1784K mutation in SCN5A is associated with mixed clinical phenotype of type 3 long QT syndrome. J Clin Invest 118: 2219-2229

Malan D, Friedrichs S, Fleischmann BK, Sasse P (2011). Cardiomyocytes obtained from induced pluripotent stem cells with long-QT syndrome 3 recapitulate typical disease-specific features in vitro. Circ Res 109: 841-847

Mali P, Yang L, Esvelt KM, Aach J, Guell M, DiCarlo JE, Norville JE, Church GM (2013). RNA-guided human genome engineering via Cas9. Science 339: 823-826

Márquez MF, Salica G, Hermosillo AG, Pastelín G, Cárdenas M (2005). Drug therapy in Brugada syndrome. Curr Drug Targets Cardiovasc Haematol Disord 5: 409-417 
Márquez MF, Salica G, Hermosillo AG, Pastelín G, Gómez-Flores J, Nava S, Cárdenas $\mathbf{M}$ (2007). Ionic basis of pharmacological therapy in Brugada syndrome. $J$ Cardiovasc Electrophysiol 18: 234-240

Martin CA, Zhang Y, Grace AA, Huang CL (2010). Increased right ventricular repolarization gradients promote arrhythmogenesis in a murine model of Brugada syndrome. J Cardiovasc Electrophysiol 21: 1153-1159

Martin GR (1981). Isolation of a pluripotent cell line from early mouse embryos cultured in medium conditioned by teratocarcinoma stem cells. Proc Natl Acad Sci U S A 78: 76347638

Masui S, Nakatake Y, Toyooka Y, Shimosato D, Yagi R, Takahashi K, Okochi H, Okuda A, Matoba R, Sharov AA, Ko MS, Niwa H (2007). Pluripotency governed by Sox2 via regulation of Oct3/4 expression in mouse embryonic stem cells. Nat Cell Biol 9: 625-635

Matsa E, Rajamohan D, Dick E, Young L, Mellor I, Staniforth A, Denning C (2011). Drug evaluation in cardiomyocytes derived from human induced pluripotent stem cells carrying a long QT syndrome type 2 mutation. Eur Heart J 32: $952-962$

Maury P, Couderc P, Delay M, Boveda S, Brugada J (2004). Electrical storm in Brugada syndrome successfully treated using isoprenaline. Europace 6: 130-133

Mayshar Y, Ben-David U, Lavon N, Biancotti JC, Yakir B, Clark AT, Plath K, Lowry WE, Benvenisty N (2010). Identification and classification of chromosomal aberrations in human induced pluripotent stem cells. Cell Stem Cell 7: 521-531

Meregalli PG, Wilde AA, Tan HL (2005). Pathophysiological mechanisms of Brugada syndrome: depolarization disorder, repolarization disorder, or more? Cardiovasc Res 67: $367-378$

Mingeot-Leclercq MP, Tulkens PM (1999). Aminoglycosides: Nephrotoxicity. Antimicrob Agents Chemother 43: 1003-1012

Mitsui K, Tokuzawa Y, Itoh H, Segawa K, Murakami M, Takahashi K, Maruyama M, Maeda M, Yamanaka S (2003). The homeoprotein Nanog is required for maintenance of pluripotency in mouse epiblast and ES cells. Cell 113: 631-642

Mizusawa Y, Wilde AAM (2012). Brugada syndrome. Circ Arrhythm Electrophysiol 5: 606616

Mok NS, Chan NY, Chiu AC (2004). Successful use of quinidine in treatment of electrical storm in Brugada syndrome. Pacing Clin Electrophysiol 27: 821-823

Moretti A, Bellin M, Welling A, Jung CB, Lam JT, Bott-Flügel L, Dorn T, Goedel A, Höhnke C, Hofmann F, Seyfarth M, Sinnecker D, Schömig A, Laugwitz KL (2010). Patient-specific induced pluripotent stem-cell models for long-QT syndrome. $N$ Engl J Med. 363: 1397-1409 
Motoike HK, Liu H, Glaaser IW, Yang AS, Tateyama M, Kass RS (2004). The $\mathrm{Na}^{+}$ channel inactivation gate is a molecular complex: a novel role of the $\mathrm{COOH}$-terminal domain. J Gen Physiol 123: 155-165

Nademanee K, Veerakul G, Chandanamattha P, Chaothawee L, Ariyachaipanich A, Jirasirirojanakorn K, Likittanasombat $K$, Bhuripanyo $K$, Ngarmukos $T$ (2011). Prevention of ventricular fibrillation episodes in Brugada syndrome by catheter ablation over the anterior right ventricular outflow tract epicardium. Circulation 123: 1270-1279

Nagatomo T, Fan Z, Ye B, Tonkovich GS, January CT, Kyle JW, Makielski JC (1998). Temperature dependence of early and late currents in human cardiac wild-type and long Q-T DeltaKPQ Na ${ }^{+}$channels. Am J Physiol 275: H2016-2024

Nicholas CR, Kriegstein AR (2010). Regenerative medicine: Cell reprogramming gets direct. Nature 463: 1031-1032

Novak A, Shtrichman R, Germanguz I, Segev H, Zeevi-Levin N, Fishman B, Mandel YE, Barad L, Domev H, Kotton D, Mostoslavsky G, Binah O, Itskovitz-Eldor J (2010). Enhanced reprogramming and cardiac differentiation of human keratinocytes derived from plucked hair follicles, using a single excisable lentivirus Cell Reprogram 12: 665-678

Novak A, Barad L, Zeevi-Levin N, Shick R, Shtrichman R, Lorber A, Itskovitz-Eldor J, Binah $\mathrm{O}$ (2012). Cardiomyocytes generated from CPVTD307H patients are arrhythmogenic in response to $\beta$-adrenergic stimulation. J Cell Mol Med 16: 468-482

Nuyens D, Stengl M, Dugarmaa S, Rossenbacker T, Compernolle V, Rudy Y, Smits JF, Flameng W, Clancy CE, Moons L, Vos MA, Dewerchin M, Benndorf K, Collen D, Carmeliet E, Carmeliet P (2001). Abrupt rate accelerations or premature beats cause lifethreatening arrhythmias in mice with long-QT3 syndrome. Nat Med 7: 1021-1027

Obokata H, Wakayama T, Sasai Y, Kojima K, Vacanti MP, Niwa H, Yamato M, Vacanti CA. (2014). Stimulus-triggered fate conversion of somatic cells into pluripotency. Nature 505: 641-647

Obokata H, Sasai Y, Niwa H, Kadota M, Andrabi M, Takata N, Tokoro M, Terashita Y, Yonemura S, Vacanti CA, Wakayama T (2014). Bidirectional developmental potential in reprogrammed cells with acquired pluripotency. Nature 505: 676-680

Okita K, Ichisaka T, Yamanaka S (2007). Generation of germ-line competent induced pluripotent stem cells. Nature 448: 313-317

Okita K, Nakagawa M, Hyenjong H, Ichisaka T, Yamanaka S (2008). Generation of mouse induced pluripotent stem cells without viral vectors. Science 322: 949-953

Papadatos GA, Wallerstein PM, Head CE, Ratcliff R, Brady PA, Benndorf K, Saumarez RC, Trezise AE, Huang CL, Vandenberg JI, Colledge WH, Grace AA (2002). Slowed conduction and ventricular tachycardia after targeted disruption of the cardiac sodium channel gene Scn5a. Proc Natl Acad Sci U S A 99: 6210-6215 
Pesce M, Wang X, Wolgemuth DJ, Schöler H (1998). Differential expression of the Oct-4 transcription factor during mouse germ cell differentiation. Mech Dev 71: 89-98

Petitprez S, Jespersen T, Pruvot E, Keller DI, Corbaz C, Schläpfer J, Abriel H, Kucera JP (2008). Analyses of a novel SCN5A mutation (C1850S): conduction vs. repolarization disorder hypotheses in the Brugada syndrome. Cardiovasc Res 78: 494-504

Priori SG, Wilde AA, Horie M, Cho Y, Behr ER, Berul C, Blom N, Brugada J, Chiang CE, Huikuri H, Kannankeril P, Krahn A, Leenhardt A, Moss A, Schwartz PJ, Shimizu W, Tomaselli G, Tracy C (2013). HRS/EHRA/APHRS expert consensus statement on the diagnosis and management of patients with inherited primary arrhythmia syndromes: document endorsed by HRS, EHRA, and APHRS in May 2013 and by ACCF, AHA, PACES, and AEPC in June 2013. Heart Rhythm 10: 1932-1963

Remme CA, Verkerk AO, Nuyens D, van Ginneken AC, van Brunschot S, Belterman CN, Wilders R, van Roon MA, Tan HL, Wilde AA, Carmeliet P, de Bakker JM, Veldkamp MW, Bezzina CR (2006). Overlap syndrome of cardiac sodium channel disease in mice carrying the equivalent mutation of human SCN5A-1795insD. Circulation 114: 2584-2594

Richards M, Tan SP, Tan JH, Chan WK, Bongso A (2004). The transcriptome profile of human embryonic stem cells as defined by SAGE. Stem Cells 22: 51-64

Rodda DJ, Chew JL, Lim LH, Loh YH, Wang B, Ng HH, Robson P (2005). Transcriptional regulation of nanog by OCT4 and SOX2. J Biol Chem 280: 24731-24737

Rook MB, Evers MM, Vos MA, Bierhuizen MF (2012). Biology of cardiac sodium channel Nav1.5 expression. Cardiovasc Res 93: 12-23

Rosner MH, Vigano MA, Ozato K, Timmons PM, Poirier F, Rigby PW, Staudt LM (1990). A POU-domain transcription factor in early stem cells and germ cells of the mammalian embryo. Nature 345: 686-92

Ruan Y, Liu N, Priori SG (2009). Sodium channel mutations and arrhythmias. Nat Rev Cardiol 6: 337-348

Sartiani L, Bettiol E, Stillitano F, Mugelli A, Cerbai E, Jaconi ME (2007). Developmental changes in cardiomyocytes differentiated from human embryonic stem cells: a molecular and electrophysiological approach. Stem Cells 25: 1136-1144

Satin J1, Kehat I, Caspi O, Huber I, Arbel G, Itzhaki I, Magyar J, Schroder EA, Perlman I, Gepstein L (2004). Mechanism of spontaneous excitability in human embryonic stem cell derived cardiomyocytes. J Physiol 559: 479-496

Sato N, Sanjuan IM, Heke M, Uchida M, Naef F, Brivanlou AH (2003). Molecular signature of human embryonic stem cells and its comparison with the mouse. Dev Biol 260: 404-413 
Schulze-Bahr E, Eckardt L, Breithardt G, Seidl K, Wichter T, Wolpert C, Borggrefe M, Haverkamp W (2003). Sodium channel gene (SCN5A) mutations in 44 index patients with Brugada syndrome: different incidences in familial and sporadic disease. Hum Mutat 21: 651-652

Schwartz PJ, Priori SG, Spazzolini C, Moss AJ, Vincent GM, Napolitano C, Denjoy I, Guicheney P, Breithardt G, Keating MT, Towbin JA, Beggs AH, Brink P, Wilde AA, Toivonen L, Zareba W, Robinson JL, Timothy KW, Corfield V, Wattanasirichaigoon D, Corbett C, Haverkamp W, Schulze-Bahr E, Lehmann MH, Schwartz K, Coumel P, Bloise $\mathbf{R}$ (2001). Genotype-phenotype correlation in the long-QT syndrome: gene-specific triggers for life-threatening arrhythmias. Circulation 103: 89-95

Shefner JM, Cudkowicz ME, Schoenfeld D, Conrad T, Taft J, Chilton M, Urbinelli L, Qureshi M, Zhang H, Pestronk A, Caress J, Donofrio P, Sorenson E, Bradley W, Lomen-Hoerth C, Pioro E, Rezania K, Ross M, Pascuzzi R, Heiman-Patterson T, Tandan R, Mitsumoto H, Rothstein J, Smith-Palmer T, MacDonald D, Burke D; NEALS Consortium (2004). A clinical trial of creatine in ALS. Neurology 63: 1656-1661

Somers A, Jean JC, Sommer CA, Omari A, Ford CC, Mills JA, Ying L, Sommer AG, Jean JM, Smith BW, Lafyatis R, Demierre MF, Weiss DJ, French DL, Gadue P, Murphy GJ, Mostoslavsky G, Kotton DN (2010). Generation of transgene-free lung disease-specific human induced pluripotent stem cells using a single excisable lentiviral stem cell cassette. Stem Cells 28: 1728-1740

Stadtfeld M, Nagaya M, Utikal J, Weir G, Hochedlinger K (2008). Induced pluripotent stem cells generated without viral integration. Science 322: 945-949

Stem Cells: Scientific Progress and Future Research Directions. Department of Health and Human Services. June 2001

Streckfuss-Bömeke K, Wolf F, Azizian A, Stauske M, Tiburcy M, Wagner S, Hübscher D, Dressel R, Chen S, Jende J, Wulf G, Lorenz V, Schön MP, Maier LS, Zimmermann WH, Hasenfuss G, Guan K (2013). Comparative study of human-induced pluripotent stem cells derived from bone marrow cells, hair keratinocytes, and skin fibroblasts. Eur Heart J 34: 2618-2629.

Stühmer W, Conti F, Suzuki H, Wang XD, Noda M, Yahagi N, Kubo H, Numa S (1989). Structural parts involved in activation and inactivation of the sodium channel. Nature 339: 597-603

Tada M, Takahama Y, Abe K, Nakatsuji N, Tada T (2001). Nuclear reprogramming of somatic cells by in vitro hybridization with ES cells. Curr Biol 11: 1553-1558

Takahashi K, Yamanaka S (2006). Induction of pluripotent stem cells from mouse embryonic and adult fibroblast cultures by defined factors. Cell 126: 663-676 
Takahashi K, Tanabe K, Ohnuki M, Narita M, Ichisaka T, Tomoda K, Yamanaka S (2007). Induction of pluripotent stem cells from adult human fibroblasts by defined factors. Cell 131: 861-872

Teng S, Gao L, Paajanen V, Pu J, Fan Z (2009). Readthrough of nonsense mutation W822X in the SCN5A gene can effectively restore expression of cardiac $\mathrm{Na}^{+}$channels. Cardiovasc Res 83: 473-480

Terrenoire C, Wang K, Tung KW, Chung WK, Pass RH, Lu JT, Jean JC, Omari A, Sampson KJ, Kotton DN, Keller G, Kass RS (2013). Induced pluripotent stem cells used to reveal drug actions in a long QT syndrome family with complex genetics. J Gen Physiol 141: $61-72$

The Nobel Prize in Physiology or Medicine 2012: press release $\left(8^{\text {th }}\right.$ October 2012). http://www.nobelprize.org/nobel_prizes/medicine/laureates/2012/press.html

Thomson JA, Itskovitz-Eldor J, Shapiro SS, Waknitz MA, Swiergiel JJ, Marshall VS, Jones JM (1998). Embryonic stem cell lines derived from human blastocysts. Science 282: $1145-1147$

Tohyama S, Hattori F, Sano M, Hishiki T, Nagahata Y, Matsuura T, Hashimoto H, Suzuki T, Yamashita H, Satoh Y, Egashira T, Seki T, Muraoka N, Yamakawa H, Ohgino Y, Tanaka T, Yoichi M, Yuasa S, Murata M, Suematsu M, Fukuda K (2013). Distinct metabolic flow enables large-scale purification of mouse and human pluripotent stem cell-derived cardiomyocytes. Cell Stem Cell 12: 127-137

Tomioka M, Nishimoto M, Miyagi S, Katayanagi T, Fukui N, Niwa H, Muramatsu M, Okuda A (2002). Identification of Sox-2 regulatory region which is under the control of Oct-3/4-Sox-2-complex. Nucleic Acids Res 30: 3202-3213

Veerakul G, Nademanee K (2012). Brugada syndrome: two decades of progress. Circ J 76: 2713-2722

Veldkamp MW, Viswanathan PC, Bezzina C, Baartscheer A, Wilde AA, Balser JR (2000). Two distinct congenital arrhythmias evoked by a multidysfunctional $\mathrm{Na}(+)$ channel. Circ Res 86: E91-97

Vierbuchen T, Ostermeier A, Pang ZP, Kokubu Y, Südhof TC, Wernig M (2010). Direct conversion of fibroblasts to functional neurons by defined factors. Nature 463: 1035-1041

Viskin S1, Wilde AA, Guevara-Valdivia ME, Daoulah A, Krahn AD, Zipes DP, Halkin A, Shivkumar K, Boyle NG, Adler A, Belhassen B, Schapachnik E, Asrar F, Rosso R, Fadreguilan EC, Veltman C, Veerakul G, Marquez M, Juneja R, Daoulah AN, Caorsi WR, Cuesta A, Jensen HK, Hamad AK, Spears D, Lozano IF, Urda VC, Peinado R, Panduranga P, Emkanjoo Z, Bergfeldt L, Janousek J (2013). Quinidine, a life-saving medication for Brugada syndrome, is inaccessible in many countries. J Am Coll Cardiol 61: 2383-2387 
Wakayama T, Yanagimachi $\mathbf{R}$ (1999). Cloning of male mice from adult tail-tip cells. Nat Genet 22: 127-128

Wang DW, Makita N, Kitabatake A, Balser JR, George AL Jr (2000). Enhanced Na(+) channel intermediate inactivation in Brugada syndrome. Circ Res 87: E37-43.

Wang Q, Shen J, Splawski I, Atkinson D, Li Z, Robinson JL, Moss AJ, Towbin JA, Keating MT (1995). SCN5A mutations associated with an inherited cardiac arrhythmia, long QT syndrome. Cell 80: 805-811

Wang Y, Zhang WY, Hu S, Lan F, Lee AS, Huber B, Lisowski L, Liang P, Huang M, de Almeida PE, Won JH, Sun N, Robbins RC, Kay MA, Urnov FD, Wu JC (2012). Genome editing of human embryonic stem cells and induced pluripotent stem cells with zinc finger nucleases for cellular imaging. Circ Res 111: 1494-1503

Warren L, Manos PD, Ahfeldt T, Loh YH, Li H, Lau F, Ebina W, Mandal PK, Smith ZD, Meissner A, Daley GQ, Brack AS, Collins JJ, Cowan C, Schlaeger TM, Rossi DJ (2010). Highly efficient reprogramming to pluripotency and directed differentiation of human cells with synthetic modified mRNA. Cell Stem Cell 7: 618-630

Watanabe H, Yang T, Stroud DM, Lowe JS, Harris L, Atack TC, Wang DW, Hipkens SB, Leake B, Hall L, Kupershmidt S, Chopra N, Magnuson MA, Tanabe N, Knollmann BC, George AL Jr, Roden DM (2011). Striking In vivo phenotype of a disease-associated human SCN5A mutation producing minimal changes in vitro. Circulation 124: 1001-1011

Wattanasirichaigoon D, Vesely MR, Duggal P, Levine JC, Blume ED, Wolff GS, Edwards SB, Beggs AH (1999). Sodium channel abnormalities are infrequent in patients with long QT syndrome: identification of two novel SCN5A mutations. Am J Med Genet 86: 470-476

Wei J, Wang DW, Alings M, Fish F, Wathen M, Roden DM, George AL Jr (1999). Congenital long-QT syndrome caused by a novel mutation in a conserved acidic domain of the cardiac $\mathrm{Na}^{+}$channel. Circulation 99: 3165-3171

Welch EM, Barton ER, Zhuo J, Tomizawa Y, Friesen WJ, Trifillis P, Paushkin S, Patel M, Trotta CR, Hwang S, Wilde RG, Karp G, Takasugi J, Chen G, Jones S, Ren H, Moon YC, Corson D, Turpoff AA, Campbell JA, Conn MM, Khan A, Almstead NG, Hedrick J, Mollin A, Risher N, Weetall M, Yeh S, Branstrom AA, Colacino JM, Babiak J, Ju WD, Hirawat S, Northcutt VJ, Miller LL, Spatrick P, He F, Kawana M, Feng H, Jacobson A, Peltz SW, Sweeney HL (2007). PTC124 targets genetic disorders caused by nonsense mutations. Nature 447: 87-91

Wernig M, Meissner A, Foreman R, Brambrink T, Ku M, Hochedlinger K, Bernstein BE, Jaenisch R (2007). In vitro reprogramming of fibroblasts into a pluripotent ES-cell-like state. Nature 448: 318-324 
West JW, Patton DE, Scheuer T, Wang Y, Goldin AL, Catterall WA (1992). A cluster of hydrophobic amino acid residues required for fast $\mathrm{Na}^{+}$-channel inactivation. Proc Natl Acad Sci U S A 89: 10910-10914

Wilde AA, Antzelevitch C, Borggrefe M, Brugada J, Brugada R, Brugada P, Corrado D, Hauer RN, Kass RS, Nademanee K, Priori SG, Towbin JA; Study Group on the Molecular Basis of Arrhythmias of the European Society of Cardiology (2002). Proposed diagnostic criteria for the Brugada syndrome: consensus report. Circulation 106: 2514-2519

Wilde AA, Postema PG, Di Diego JM, Viskin S, Morita H, Fish JM, Antzelevitch C (2010). The pathophysiological mechanism underlying Brugada syndrome: depolarization versus repolarization. J Mol Cell Cardiol 49: 543-553

Wilde AAM, Bezzina CR (2005). Genetics of cardiac arrhythmias. Heart 91: 1352-1358

Wilmut I, Schnieke AE, McWhir J, Kind AJ, Campbell KH (1997). Viable offspring derived from fetal and adult mammalian cells. Nature 385: 810-813

Wolf CM, Berul CI (2008). Molecular mechanisms of inherited arrhythmias. Curr Genomics 9: $160-168$

Xu C, Police S, Rao N, Carpenter MK (2002). Characterization and enrichment of cardiomyocytes derived from human embryonic stem cells. Circ Res 91: 501-508

Xu N, Papagiannakopoulos T, Pan G, Thomson JA, Kosik KS (2009). MicroRNA-145 regulates $O C T 4, S O X 2$, and $K L F 4$ and represses pluripotency in human embryonic stem cells. Cell 137: 647-658

Yan GX, Shimizu W, Antzelevitch C (1998). Characteristics and distribution of M cells in arterially perfused canine left ventricular wedge preparations. Circulation 98: 1921-1927

Yan GX, Antzelevitch C (1999). Cellular basis for the Brugada syndrome and other mechanisms of arrhythmogenesis associated with ST-segment elevation. Circulation 100: $1660-1666$

Yang F, Hanon S, Lam P, Schweitzer P (2009). Quinidine revisited. Am J Med 122: 317321.

Yazawa M, Hsueh B, Jia X, Pasca AM, Bernstein JA, Hallmayer J, Dolmetsch RE (2011). Using induced pluripotent stem cells to investigate cardiac phenotypes in Timothy syndrome. Nature 471: 230-234

Ye L, Muench MO, Fusaki N, Beyer AI, Wang J, Qi Z, Yu J, Kan YW (2013). Blood cellderived induced pluripotent stem cells free of reprogramming factors generated by sendai viral vectors. Stem Cells Transl Med 2: 558-566 
Yu J, Vodyanik MA, Smuga-Otto K, Antosiewicz-Bourget J, Frane JL, Tian S, Nie J, Jonsdottir GA, Ruotti V, Stewart R, Slukvin II, Thomson JA (2007). Induced pluripotent stem cell lines derived from human somatic cells. Science 318: 1917-1920

Zareba W, Sattari MN, Rosero S, Couderc JP, Moss AJ (2001). Altered atrial, atrioventricular, and ventricular conduction in patients with the long QT syndrome caused by the DeltaKPQ SCN5A sodium channel gene mutation. Am J Cardiol 88: 1311-1314

Zhang J, Wilson GF, Soerens AG, Koonce CH, Yu J, Palecek SP, Thomson JA, Kamp TJ (2009). Functional cardiomyocytes derived from human induced pluripotent stem cells. Circ Res 104: 30-41

Zhang WY, de Almeida PE, Wu JC (2008). Teratoma formation: A tool for monitoring pluripotency in stem cell research. In: StemBook (Girard L ed.), Harvard Stem Cell Institute, Cambridge, MA

Zhou H, Wu S, Joo JY, Zhu S, Han DW, Lin T, Trauger S, Bien G, Yao S, Zhu Y, Siuzdak G, Schöler HR, Duan L, Ding S (2009). Generation of induced pluripotent stem cells using recombinant proteins. Cell Stem Cell 4: 381-384

Zwi-Dantsis L, Huber I, Habib M, Winterstern A, Gepstein A, Arbel G, Gepstein L (2013). Derivation and cardiomyocyte differentiation of induced pluripotent stem cells from heart failure patients. Eur Heart J 34: 1575-1586 


\section{Acknowledgements}

I would like to take the opportunity to express my deepest gratitude to all people who supported me during my doctoral studies over the last years.

Firstly, I would like to thank my thesis committee members Prof. Dr. Ernst A. Wimmer, Prof. Dr. med. Dr. h. c. Wolfgang Engel, and PD Dr. Kaomei Guan-Schmidt for their scientific support and helpful discussions in our annual committee meetings. A special thanks goes to PD Dr. Kaomei Guan-Schmidt for giving me the opportunity to be a doctoral student under her supervision and for always lending a listening ear. In addition, a deep appreciation to Prof. Dr. med. Gerd Hasenfuß who gave me the chance to work for my doctoral thesis in the Department of Cardiology and Pneumology at the University Medical Center Göttingen.

I would like to extend my appreciation to the GGNB for supporting me financially with a travel grant and for giving me the opportunity to get an insight into various methods and skills courses. A big thank you goes to the GGNB office for their dedicated coordination of all administrative procedures.

I am also very grateful to Thomas Sowa, Dr. med. Stefan Wagner, Azadeh Azizian, and Prof. Dr. med. Lars Maier for the great collaboration and especially for teaching me the patch clamp technique. Thank you to Mohammad Hashemiansab and Dr. Mladen Tzvetkov for being so enthusiastic in helping me with the next generation sequencing experiments.

I would also like to acknowledge all the individuals for donating their cells for my $\mathrm{PhD}$ project.

I owe particular thanks to all my helpful colleagues from the stem cell lab for the friendly and very nice atmosphere in the lab. Special thanks go to Yvonne Wiegräfe, Wener Li, Yvonne Hintz, and Katrin Streckfuß-Bömeke for their helpful assistance.

Last but not least I want to thank my family for their moral support during the last years. You always encouraged me to go on, especially during the difficult periods of my $\mathrm{PhD}$ thesis. A big thank you and a big hug go also to Simin for her everlasting patience and loving support (especially for counting so many chromosomes (:)). I am very glad that I met you here in Göttingen! 